\title{
CONTROLLING SYNCHRONIZATION PATTERNS IN COMPLEX NETWORKS
}

\author{
vorgelegt von \\ Diplom-Physikerin \\ Anica Judith Lehnert \\ aus Berlin \\ von der Fakultät II - Mathematik und Naturwissenschaften -

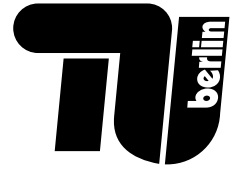 \\ der Technischen Universität Berlin \\ zur Erlangung des akademischen Grades \\ Doktor der Naturwissenschaften \\ - Dr. rer. nat. - \\ genehmigte Dissertation
}

Promotionsausschuss:

Vorsitzender: Prof. Dr. Stephan Reitzenstein

Berichter: $\quad$ Prof. Dr. Eckehard Schöll, PhD

Berichter: $\quad$ PD Dr. Serhiy Yanchuk

Tag der wissenschaftlichen Aussprache: 24. März 2015

Berlin 2015

D 83 



\section{ABSTRACT}

In this thesis, I consider the control of synchronization in delay-coupled complex networks. As one main focus, several applications to neural networks will be discussed. In the first part, I focus on the stability of synchronization in complex networks meaning that the control is realized by considering the stability of synchrony in dependence on the parameters. In the second part, adaptive control of synchronization is studied. To this end, adaptive control algorithms are developed that tune the system parameters such that the desired control goal is reached.

Besides zero-lag synchrony - a state where all nodes follow the same dynamics without a phase lag - groups and cluster states are considered, i.e., states where the network consists of several groups where the nodes within one group are in zero-lag synchrony and, in the case of cluster synchrony, with a constant phase lag between the clusters.

The stability of synchronization can be accessed via the master stability function [Pecora and Carroll, 1998]. This convenient tool allows for treating the node dynamics and the network topology in two separate steps, and, thus, allows for a quite general treatment of different network topologies. In this thesis, I will discuss the generalization of the master stability function to group and cluster states and to non-smooth systems.

The master stability function can be used to investigate synchrony in neural networks. Neurons are excitable systems where type-I and type-II excitability can be distinguished. Here, the stability of synchrony for both types in complex networks with excitatory and inhibitory links is investigated on two generic models, namely the normal form of the saddle-node infinite period bifurcation and the FitzHugh-Nagumo system. Furthermore, synchronization in systems with heterogeneous delays or node parameters is studied.

In situations where parameters are unknown or drift, adaptive control methods are useful since they allow for an automatically realized adaption of the control parameters. A convenient adaptive method is the speed-gradient method that minimizes a predefined goal function [Fradkov, 1979, 2007]. I first apply this method in the control of an unstable focus and an unstable periodic orbit embedded in the Rössler attractor. Furthermore, I show that clusters states in networks of delayed coupled Stuart-Landau oscillators can be controlled by adaptively tuning the phase of the complex coupling strength or by adapting the topology. The first method is particularly simple because only one parameter has to be adapted, while the second method is more reliable in the sense that its success is widely independent of the choice of the control parameters and the initial conditions. 


\section{ZUSAMMENFASSUNG}

In dieser Arbeit wird die Kontrolle von Synchronisation in komplexen Netzwerken mit retardierten Kopplungen untersucht. Dabei werden verschiedene Anwendungen auf neuronale Netzwerke diskutiert. Der erste Teil der Arbeit beschäftigt sich mit der Stabilität von Synchronisation in komplexen Netzwerken, wobei der Kontrollaspekt dadurch verwirklicht wird, dass die Stabilität in Abhängigkeit der Parameter betrachtet wird. Im Fokus des zweiten Teiles steht die adaptive Kontrolle von Synchronisation. Zu diesem Zweck werden adaptive Kontrollalgorithmen entwickelt, die die Systemparameter so anpassen, dass das gewünschte Kontrollziel verwirklicht wird.

Neben Zero-lag-Synchronisation, einem Zustand, in dem alle Knoten der gleichen Dynamik ohne eine Phasenverschiebung folgen, werden Gruppen- und Clusterzustände betachtet. Dabei handelt es sich um Zustände, bei denen sich die Knoten innerhalb einer Gruppe in Zero-lag-Synchronisation befinden mit - im Fall von von Clustersynchronisation - einem konstanten Phasenunterschied zueinander.

Die Stabilität von Synchronisation kann mit Hilfe der Master Stability Function (MSF) berechnet werden [Pecora and Carroll, 1998]. Diese erlaubt es die Knotendynamik und die Topologie in zwei gesonderten Schritten zu betrachten. In dieser Arbeit werden Verallgemeinerungen der MSF auf Gruppen- und Clustersynchronisation sowie auf Systeme mit Unstetigkeitsstellen betrachtet.

Mit der MSF kann Synchronisation in neuronalen Netzwerken studiert werden. Neuronen sind anregbarer Systeme, wobei zwischen Typ-I- und Typ-II-Anregbarkeit unterschieden wird. Hier wird Synchronisation in Netzwerken mit exzitatorischer und inhibitorischer Kopplung anhand zweier für die jeweiligen Typen generischer Modelle betrachtet.

Wenn Parameter driften oder unbekannt sind, sind adaptive Kontrollemethoden nützlich, da sie für eine automatische Anpassung der Kontrollparameter sorgen. Eine bekannte adaptive Kontollmethode ist die Speed-Gradient-Methode, bei der eine zuvor definierte Zielfunktion minimiert wird [Fradkov, 1979, 2007]. Hier wird zunächst die Methode in der Stabilisierung eines instabilen Fokusses und eines instabilen Orbits erprobt. Weiterhin wird gezeigt, dass Clusterzustände in Netzwerken aus Stuart-Landau-Oszillatoren kontrolliert werden können, indem entweder die Phase der komplexen Kopplungsstärke oder die Topolgie in geeigneter Weise angepasst werden. Dabei ist die erste Methode besonders einfach, da nur ein einziger Parameter eingestellt wird, die zweite Methode aber robuster, in dem Sinne, dass sie weitestgehend unabhängig von der Wahl der Kontrollparameter und der Anfangsbedingungen ist. 


\section{PUBLICATIONS}

- Blaha, K., Lehnert, J., Keane, A., Dahms, T., Hövel, P., Schöll, E., And HudSON, J. L. (2013), Clustering in delay-coupled smooth and relaxational chemical oscillators, Phys. Rev. E 88, 062915.

- Cakan, C., Lehnert, J., And Schöll, E. (2014), Heterogeneous Delays in Neural Networks, Eur. Phys. J. B 87, 54.

- DAhms, T., Lehnert, J., AND SChÖLL, E. (2012), Cluster and group synchronization in delay-coupled networks, Phys. Rev. E 86, 016202.

- Guzenko, P. Y., LehnerT, J., AND SCHÖLL, E. (2013), Application of Adaptive Methods to Chaos Control of Networks of Rössler Systems, Cybernetics and Physics 2, 15.

- Guzenko, P. Y.,Lehnert, J., Fradkov, A. L., And SchÖll, E. (2013), Adaptive control of networks of delay-coupled chaotic systems, in Proceedings of the 6th International Conference on Physics and Control (PhysCon 2013), IPACS Electronic library.

- Keane, A., Dahms, T., Lehnert, J., Suryanarayana, S. A., Hövel, P., and Schöll, E. (2012), Synchronisation in networks of delay-coupled type-I excitable systems, Eur. Phys. J. B 85, 407.

- Ladenbauer, J., Lehnert, J., Rankoohi, H., Dahms, T., Schöll, E., And OberMAYER, K. (2013), Adaptation controls synchrony and cluster states of coupled thresholdmodel neurons, Phys. Rev. E 88, 042713.

- Lehnert, J., Hövel, P., Selivanov, A. A., Fradkov, A. L., ANd Schöll, E. (2014), Controlling cluster synchronization by adapting the topology, Phys. Rev. E 90, 042914.

- LehneRT, J., DAhMS, T., AND SCHÖLL, E. (2012), Zero-lag and group synchronization in neural networks, in Proc. 2012 Internat. Symposium on Nonlinear Theory and its Applications (NOLTA2012), Palma de Mallorca, pages 66-69, IEICE, Japan.

- Lehnert, J., Hövel, P., Flunkert, V., Guzenko, P. Y., Fradkov, A. L., ANd Schöll, E. (2011), Adaptive tuning of feedback gain in time-delayed feedback control, Chaos 21, 043111 .

- Lehnert, J., DAhms, T., Hövel, P., AND SChÖLl, E. (2011), Loss of synchronization in complex neural networks with delay, Europhys. Lett. 96, 60013. 
- Plotnikov, S. A., Lehnert, J., Fradkov, A. L., And Schöll, E. (2015), Control of synchronization in delay-coupled neural networks of heterogeneous nodes, In preparation.

- Selivanov, A. A., Lehnert, J., Fradkov, A. L., And Schöll, E. (2015), Adaptive time-delayed stabilization of steady states and periodic orbits, Phys. Rev. E 91, 012906.

- Selivanov, A. A., Lehnert, J., Dahms, T., Hövel, P., Fradkov, A. L., ANd Schöll, E. (2012), Adaptive synchronization in delay-coupled networks of Stuart-Landau oscillators, Phys. Rev. E 85, 016201.

- Schöll, E., Lehnert, J., Keane, A., Dahms, T., And Hövel, P. (2014), Control of desynchronization transitions in delay-coupled networks of type-I and type-II excitable systems, Proc. of the International Symposium, Hanse Institute of Advanced Studies, Delmenhorst, 13-16 November, 2012, in A. Pelster and G. Wunner (Editors), Selforganization in Complex Systems: The Past, Present, and Future of Synergetics, Springer, Berlin.

- Schöll, E., Lehnert, J., Dahms, T., Selivanov, A. A., And Fradkov, A. L. (2012), Adaptive time-delayed feedback control, in Proc. 2012 Internat. Symposium on Nonlinear Theory and its Applications (NOLTA2012), Palma de Mallorca, pages 674-677, IEICE, Japan.

- Schöll, E., Selivanov, A. A., Lehnert, J., Dahms, T., Hövel, P., and FradKOV, A. L. (2012), Control of synchronization in delay-coupled networks, Int. J. Mod. Phys. B 26, 1246007.

- Wille, C., LeHNERT, J., AND SCHÖLL, E. (2014), Synchronization-desynchronization transitions in complex networks: An interplay of distributed time delay and inhibitory nodes, Phys. Rev. E 90, 032908. 


\section{Contents}

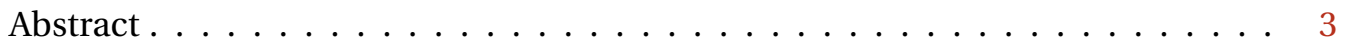

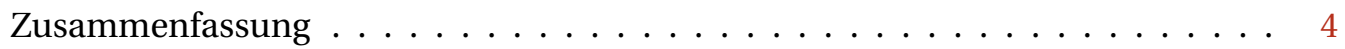

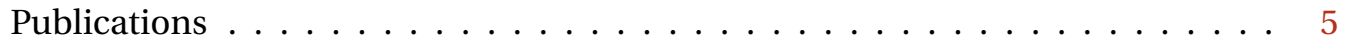

1. INTRODUCTION 11

1.1. Networks, an interdisciplinary tool . . . . . . . . . . . . . . . 11

1.2. Dynamics on networks . . . . . . . . . . . . . . . . . . 13

1.3. Adaptive control of uncoupled systems and networks . . . . . . . . . . 16

1.4. The influence of delay on the dynamics of networks . . . . . . . . . . . 17

1.5. Model systems . . . . . . . . . . . . . . . . . . 17

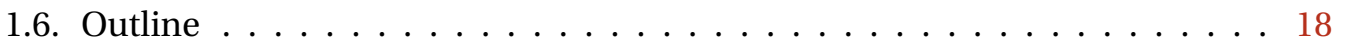

2. Complex Dynamical Networks 21

2.1. Mathematical description: From nodes and links to the coupling matrix . . 22

2.2. Network quantities . . . . . . . . . . . . . . . . 23

2.3. Network topologies . . . . . . . . . . . . . . 26

2.3.1. Regular networks with circulant matrices . . . . . . . . . . . 26

2.3.2. Erdős-Rényi random networks . . . . . . . . . . . . . . 29

2.3.3. Small-world networks . . . . . . . . . . . . . . . 30

2.3.4. Scale-free networks . . . . . . . . . . . . . . 31

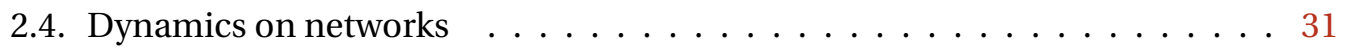

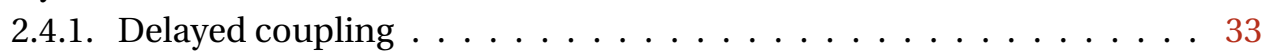

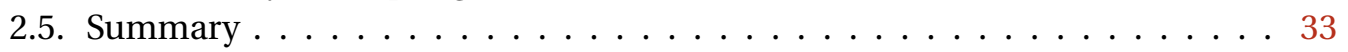

I StABILITY OF SYNCHRONIZATION

3. SYNCHRONIZATION IN COMPLEX NETWORKS

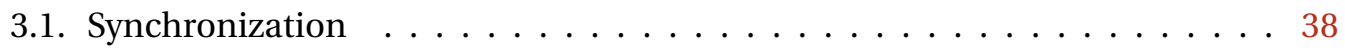


3.2. Master stability function $\ldots \ldots \ldots 39$

3.2.1. Derivation of the master stability function . . . . . . . . . . 40

3.2.2. The longitudinal eigenvalue . . . . . . . . . . . . 42

3.2.3. Symmetry of the MSF for zero-lag synchrony $\ldots \ldots \ldots 4$

3.3. Quantifying synchronization . . . . . . . . . . . . . . . . . . . . . . 44

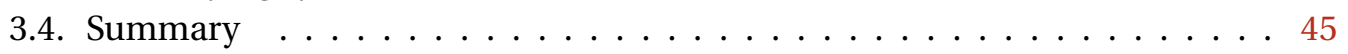

4. CONTROL OF SYNCHRONIZATION TRANSITIONS BY BALANCING EXCITATORY AND INHIBITORY COUPLING 47

4.1. Stability of excitable systems $\ldots \ldots \ldots \ldots \ldots$

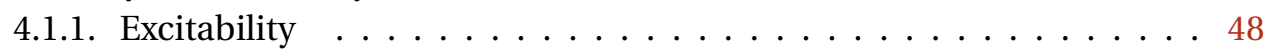

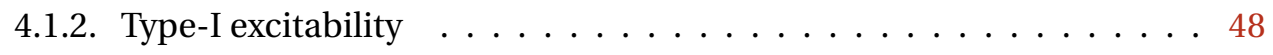

4.1.3. Type-I excitability: The master stability function . . . . . . . . . 50

4.1.4. Type-II excitability . . . . . . . . . . . . . . . . . 53

4.1.5. Type-II excitability: The master stability function . . . . . . . . . . 53

4.2. Synchronization-Desynchronization Transitions . . . . . . . . . 55

4.2.1. Network models . . . . . . . . . . . . . . . . . . 56

4.2.2. Type-II excitability for arbitrary delays and type-I excitability for large

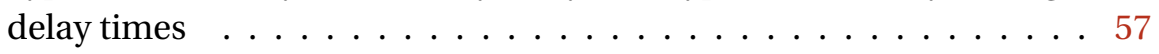

4.2.3. Type-I excitability for small delay times $\ldots \ldots$. . . . . . . . 60

4.3. Conclusion . . . . . . . . . . . . . . . . . . . . . 64

5. CluSTER AND GROUP SYNCHRONY: THE THEORY $\mathbf{6 7}$

5.1. Master stability function for group and cluster synchronization . . . . . 68

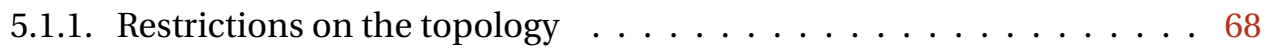

5.1.2. Derivation of the MSF for group synchrony $\ldots \ldots \ldots$. . . . . 69

5.1.3. Symmetry of the master stability function . . . . . . . . . . 75

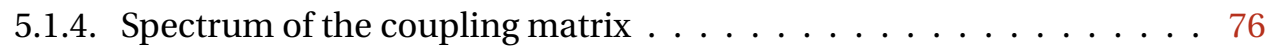

5.1.5. Beyond multipartite topologies . . . . . . . . . . . . . . . 79

5.2. The Stuart-Landau oscillator: An analytically tractable example . . . . . . 85

5.2.1. Phase of the complex coupling strength _ . . . . . . . . . 89

5.3. Non-smooth systems . . . . . . . . . . . . . . . . . . . 89

5.3.1. Master stability function for non-smooth systems _ . . . . . . . 90

5.3.2. Synchrony in coupled threshold models . . . . . . . . . . . . 94

5.4. Conclusion . . . . . . . . . . . . . . . . . . . . 100

6. ZERO-LAG AND CLUSTER SYNCHRONY: TOWARDS APPLICATIONS $\mathbf{1 0 3}$

6.1. Heterogenous delays in complex networks . . . . . . . . . . . . . . 104

6.1.1. Model . . . . . . . . . . . . . . . . . . . . . . . . . . . . . . . . 104 
6.1.2. Unimodal delay distributions in complex networks . . . . . . . . . 105

6.1.3. Two discrete delay times . . . . . . . . . . . . . . . . . . . 115

6.1.4. Bimodal delay distributions . . . . . . . . . . . . . . . . 116

6.2. Cluster states in chemical oscillators . . . . . . . . . . . . . 120

6.2.1. Experimental setup . . . . . . . . . . . . . . . . 120

6.2.2. Smooth oscillations . . . . . . . . . . . . . . . . 123

6.2.3. Relaxation oscillations . . . . . . . . . . . . . . . . . 127

6.2.4. Extended theory $\ldots \ldots \ldots \ldots \ldots$

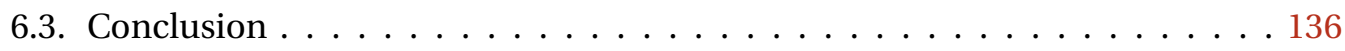

II Adaptive Control of Synchronization 139

7. ADAPTIVE CONTROL 141

7.1. Speed-gradient method . . . . . . . . . . . . . . . . . . . . 142

7.2. Summary . . . . . . . . . . . . . . . . . . . . . 144

8. ADAPTIVE TIME-DELAYED FEEDBACK CONTROL $\mathbf{1 4 5}$

8.1. Time-delayed feedback control _ . . . . . . . . . . . . . . . . 145

8.2. Stabilization of an unstable fixed point . . . . . . . . . . . . . 146

8.2.1. Robustness towards noise and drifting parameters . . . . . . . . 151

8.2.2. Extended time-delayed feedback control . . . . . . . . . . . . . . . 154

8.3. Stabilization of an unstable periodic orbit in the Rössler system . . . . . . 156

8.4. Conclusion . . . . . . . . . . . . . . . . . . . 158

9. AdAPtive CONTROL OF CLUSTER STATES IN NETWORK MOTIFS $\mathbf{1 6 1}$

9.1. Phase of the complex coupling strength . . . . . . . . . . . . . 161

9.2. Goal function for cluster synchronization . . . . . . . . . . . . . . 162

9.3. Controlling an $M$-cluster state . . . . . . . . . . . . . . . . . . . . 164

9.4. Linear stability analysis . . . . . . . . . . . . . . . . . . . 168

9.5. Performance of the adaptive control of the coupling phase . . . . . . 176

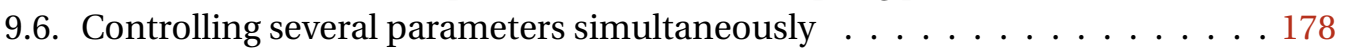

9.7. Conclusion . . . . . . . . . . . . . . . . . . . 180

10.AdAPtive TOPOLOGIES $\mathbf{1 8 3}$

10.1. Adaptive networks . . . . . . . . . . . . . . . . . . . . 184

10.2. Goal function . . . . . . . . . . . . . . . . . . 186

10.3. Adapting the topology . . . . . . . . . . . . . . . . . . . . 187

10.4. Robustness . . . . . . . . . . . . . . . . . . . . 191

10.5. Linear stability analysis $\ldots \ldots \ldots \ldots 1$ 
10.6. Structural properties _ . . . . . . . . . . . . . . . . . 193

10.6.1. Existence of cluster solutions _ . . . . . . . . . . . 195

10.6.2. Stability of cluster states _ . . . . . . . . . . . . . . . . . 197

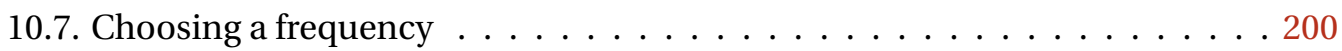

10.8. Control of a subset of links _ . . . . . . . . . . . . . . . . 204

10.9. Conclusion . . . . . . . . . . . . . . . . . 209

11. CONCLUSION 211

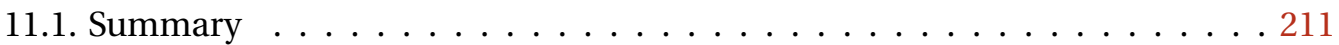

11.2. Outlook . . . . . . . . . . . . . . . . . . . 214

$\begin{array}{ll}\text { A. Delay Differential equations } & \mathbf{2 1 7}\end{array}$

$\begin{array}{lr}\text { B. KRONECKER PRODUCT } & \mathbf{2 1 9}\end{array}$

$\begin{array}{lr}\text { ACRONYMS } & \mathbf{2 2 1}\end{array}$

$\begin{array}{lc}\text { LIST OF FIGURES } & \mathbf{2 2 3}\end{array}$

$\begin{array}{lr}\text { LIST OF TABLES } & \mathbf{2 3 5}\end{array}$

$\begin{array}{ll}\text { BIBLIOGRAPHY } & \mathbf{2 3 7}\end{array}$

$\begin{array}{ll}\text { ACKNOWLEDGMENTS } & \mathbf{2 6 3}\end{array}$

$\begin{array}{lr}\text { INDEX } & \mathbf{2 6 5}\end{array}$ 


\section{1 | INTRODUCTION}

\subsection{NETWORKS, AN INTERDisCIPLINARY TOOL}

The earliest example of network science is the Königsberg problem stated and solved by the mathematician Leonhard Euler [Euler, 1741]. He asked whether it is possible to find a path that crosses each of the seven bridges of Königsberg once and only once. By describing the islands as nodes and the bridges as links connecting the nodes he found an abstract representation of the problem and laid the foundation of graph theory, the mathematical theory describing the structure of networks. He showed that it is not possible to find such a path since all four of the land masses considered in the problem are touched by an odd number of bridges. However, for a path that visits each bridge exactly once it is necessary that each land mass (except the first and the last one) will have an even number of bridges. In modern graph theory, this corresponds to the condition that each but two nodes have an even degree and is one of the first examples how network quantities can be used to characterize a network in a meaningful way.

Since the time of Euler and particularly since the 1950s network science has developed into a vivid and interdisciplinary field. Today, networks play a prominent role in the research of very different fields, ranging from social science, economics, and psychology to biology, physics, and mathematics [Boccaletti et al., 2006b; Albert and Barabasi, 2002; Newman, 2003; Newman et al., 2006; Newman, 2010], as they are a straight-forward concept to describe the interactions of many systems or agents. Several network models have been developed which share statistical properties with real-world network. In particular, random or Erdős-Rényi networks, small-world networks, scale-free networks and hierarchical networks have been in the focus of network science.

In a random network all nodes are linked with the same probability such that no correlations between the links arise [Rapoport, 1957; Solomonoff and Rapoport, 1951; Erdős and 
Rényi, 1959, 1960]. In contrast, a small-world network is characterized by a high clustering coefficient meaning that two nodes linked to the same node are much more likely to be connected to each other than two randomly chosen nodes. They are constructed from a regular network with some additional long-range connections interposed [Watts and Strogatz, 1998; Monasson, 1999; Newman and Watts, 1999]. The small-world characteristics have been encountered, among others, in metabolic networks [Jeong et al., 2000], the co-authorship of mathematicians [Barahona and Pecora, 2002], the World Wide Web [Adamic, 1999], and food webs [Montoya and Solé, 2002]. In the brain, small-world properties have been found on the level of cortical networks [Sporns et al., 2000; Sporns and Zwi, 2004; Hilgetag et al., 2000; Sporns and Zwi, 2004], in brain functional networks [Bassett et al., 2006], on the neural network level in the reticular formation of the brainstem [Humphries et al., 2006], as well as in in-vitro neural networks [Shefi et al., 2002]. Scalefree networks are characterized by the occurrence of hubs, meaning that a few nodes in the networks are connected by a large number of links, whereas most nodes have only a few links attached to them [de Solla Price, 1965; Barabasi and Albert, 1999]. Examples of scale-free networks include the World Wide Web [Albert and Barabasi, 2002], citation networks [de Solla Price, 1965], the Internet at the level of autonomous systems [Chen et al., 2002], and the interaction network of proteins [Jeong et al., 2001]. Hierarchical networks are a special type of scale free networks which are organized in a hierarchical manner into increasingly larger groups and display a high degree of clustering [Ravasz and Barabasi, 2003].

Recently, networks of networks, sometimes called multilayer networks, have gained a lot of attention [Gao et al., 2014; Boccaletti et al., 2014; Kivelä et al., 2014; Rheinwalt et al., 2014; Wiedermann et al., 2014]. They take into account that almost all real-world networks are interdependent on each other. Consider, for example, different transportation networks like flight networks, railway networks or road networks which have to interact to properly fulfill their function. Another example of highly dependent networks are biological networks like gene regulation networks, metabolic networks, and protein interaction networks. Networks of networks are highly vulnerable to node failure: The failure of a few nodes in one network can lead to a cascade of failures of dependent nodes in all networks [Buldyrev et al., 2010; Parshani et al., 2010]. A prominent example for such a cascade is the electrical blackout which hit Italy on 28 September 2003: A shutdown of power stations caused the failure of nodes in the Internet communication network which, in turn, triggered the collapse of further power stations [Buldyrev et al., 2010; Rosato et al., 2008]. Multiplex networks are a special type of multilayer networks: Here, all layers consist of the same set of nodes and each node in a layer can only be connected to its counterpart nodes in the other layers [Boccaletti et al., 2014]. The connections between the different layers represent different types of interactions between 
the nodes. Most prominently, social networks are depicted as multiplex networks. The nodes are the individuals, while the connections between the layers represent the different types of social relations possible between two individuals. Multipartite networks can be considered as another subtype of multilayer networks. A $k$-partite network consists of $k$ disjoint subsets of nodes with links between nodes of different subsets but no links between the nodes of one subset [Kivelä et al., 2014].

\subsection{DYNAMICS ON NETWORKS}

In the last two decades dynamics on network received a growing amount of interest [Dhamala et al., 2004; Zigzag et al., 2009; Choe et al., 2010; Chavez et al., 2005; Sorrentino and Ott, 2007; Albert et al., 2000; Kinzel et al., 2009; Lehnert et al., 2011a; Keane et al., 2012]. One of the central topics of dynamics on networks is synchronization [Strogatz and Stewart, 1993; Rosenblum et al., 1996; Pikovsky et al., 2001; Pecora and Carroll, 1998; Mosekilde et al., 2002; Wang and Chen, 2002; Arenas et al., 2006a, 2008; Balanov et al., 2009; Omelchenko et al., 2010; Dahms, 2011; Schöll, 2013]. Synchronization is observed in very different contexts [Arenas et al., 2008]. Biological examples include genetic oscillators [García-Ojalvo et al., 2004], circadian rhythms [Pikovsky et al., 2001; Fukuda et al., 2007], and population dynamics [Blasius et al., 1999]. In computer sciences, synchronization has been used for data mining in a large-scale database [Miyano and Tsutsui, 2007]. In automata theory and distributed computation, consensus, i.e., the ability of an ensemble of dynamic agents to reach a unique and common value, plays a major role [Olfati-Saber and Murray, 2004]. Other engineering applications where synchronization or desynchronization are of importance include wireless communication networks [Degesys et al., 2007; Diaz-Guilera et al., 2009] and power grid networks [Witthaut and Timme, 2012; Rohden et al., 2012]. Furthermore, synchronization is of interest in social sciences when it comes to opinion formation [Pluchino et al., 2005], and in the finance sector when considering the time series of stock prices [Mantegna, 1999].

Most prominently synchronization takes place in the brain where it is associated with several cognitive capacities [Fries, 2005; Uhlhaas et al., 2009]. One of these is the processing of visual input in perception [Singer, 1999; Engel et al., 2001; Singer, 2007]. According to the binding hypothesis neural synchrony is exploited to link the different features like shape, color, depth and motion of an object to form a single representation of this object. Other studies indicate that synchrony of neurons plays a role in the anticipation of an event and allows for focusing attention on this event [Murthy and Fetz, 1996; 
Roelfsema et al., 1997]. Giving the high importance of synchrony for normal functioning of the brain, it is of no surprise that several pathological brain states can be linked to abnormal synchrony. Diseases where alteration from the oscillation patterns of a healthy brain are potentially involved include Parkinson's Disease, Epilepsy, Alzheimer's Disease, Schizophrenia, and autism disorders [Poeck and Hacke, 2001; Uhlhaas et al., 2009].

This abundance of synchronization phenomena in nature and technological applications has motivated researchers to seek a fundamental understanding of synchronization and, in particular, of the interplay between synchrony and network topology [Pecora and Carroll, 1998; Belykh et al., 2005; Arenas et al., 2006a; Gutiérrez et al., 2011; Sorrentino and Ott, 2007; D’Huys et al., 2013; Flunkert et al., 2010; Pecora et al., 2014]. Maybe the most profound step in this direction has been taken by Pecora and Carroll [1998] in developing the master stability function (MSF), a convenient tool to calculate the stability of synchronization in complex networks. The MSF allows for considering the local dynamics of the nodes and the network topology separately. The power of the MSF is that not single network realizations have to be considered but that whole classes of network topologies can be studied by investigating their eigenvalue spectrum. On several occasions the master stability function has been used to study synchrony in neural networks [Dhamala et al., 2004; Rossoni et al., 2005; Belzig, 2005; Lehnert et al., 2011a; Keane et al., 2012]. Frequently, models employed in neuroscience are of integrate-and-fire type meaning that the membrane voltage is set back to a resting potential after reaching a threshold. This gives rise to a discontinuity in the time series. In [Ladenbauer et al., 2013], we derived an MSF for these non-smooth systems.

Besides zero-lag synchronization, a state where all nodes undergo the same dynamics without a phase shift, group and cluster synchronization have received growing interest both in theory [Sorrentino and Ott, 2007; Kestler et al., 2007, 2008; Dahms et al., 2012; Kanter et al., 2011b,a; Golubitsky and Stewart, 2002; Lücken and Yanchuk, 2012; Sorrentino, 2014; Pecora et al., 2014; Poel et al., 2015] and in experiments [Illing et al., 2011; Aviad et al., 2012; Blaha et al., 2013; Williams et al., 2012, 2013a; Rosin, 2015]. In the case of group synchrony, the network consists of several groups where the nodes within one group are in zero-lag synchrony [Dahms, 2011; Dahms et al., 2012]. Between the groups no synchrony exists, or in the case of clusters states, synchrony with a constant phase shift is observed. Group and cluster synchronization occurs, among others, in dynamics of nephrons [Mosekilde et al., 2002], central pattern generation in animal locomotion [Ijspeert, 2008], or population dynamics [Blasius et al., 1999]. As in the case of zerolag synchrony, group and cluster synchronization can be treated within the framework of the MSF: Sorrentino and Ott [2007] extended the MSF to two groups, while we gave generalization to an arbitrary number of groups in [Lehnert, 2010; Dahms, 2011; Dahms 
et al., 2012]. In [Pecora et al., 2014], a very general answer to the question which kinds of topologies exhibit states of group synchrony and a discussion of the stability of these states via a MSF is given. In [Ladenbauer et al., 2013], group synchrony in non-smooth systems was considered.

In neural networks, inhibitory processes play a key role [Okun and Lampl, 2009; Belykh and Shilnikov, 2008; Vogels and Abbott, 2009; Henderson and Robinson, 2011; Deco et al., 2014]. Without inhibitory neurons counteracting the excitatory ones, encoding and processing of information would be infeasible since excitation would always create more excitation [Okun and Lampl, 2009; Jonas and Buzsáki, 2007]. In the sensory system, for example, lateral inhibition confines the activity of a neural group by suppressing the activity of neighboring neurons which are less active. In this way, the contrast of a stimulus is enhanced [Kandel et al., 1996]. In epilepsy, a lack of inhibition, for example, caused by an attenuation of the chief inhibitory neurotransmitter gamma-Aminobutyric acid, leads to pathological synchronized activity of neurons, which normally act asynchronously [Poeck and Hacke, 2001].

Within the framework of the MSF, two possibilities exist to take inhibitory processes into account: (i) A simple approach is to consider negative weights in the coupling matrix of the network, while (ii) the MSF for group synchrony allows for applying a more sophisticated approach where groups of excitatory and inhibitory neurons are considered. In [Lehnert et al., 2011a; Keane et al., 2012; Schöll et al., 2014], we used the first approach to study synchronization-desynchronization transitions in neural networks with inhibition. The second approach was applied in [Ladenbauer et al., 2013] when considering group synchrony in networks of adaptive integrate-and-fire models.

The MSF is only applicable to networks of identical nodes and only if the delay between all node pairs is identical, where the delay can be one discrete delay time [Dhamala et al., 2004; Lehnert et al., 2011a; Dahms, 2011; Keane et al., 2012; Schöll, 2013] or a delay kernel [Kyrychko et al., 2014; Wille et al., 2014]. Only in the case of commuting coupling matrices two or several discrete delay times can be taken into account [Dahms, 2011; Dahms et al., 2012]. However, real-world networks are often poorly described by commuting coupling matrices, discrete delay times, and identical nodes, but will always be characterized by a certain degree of heterogeneity in the network structure as well as in the parameters. Therefore, the question arises how well the MSF predicts the stability of synchrony in situations characterized by the presence of heterogeneities.

We, therefore, extended the previous works [Lehnert et al., 2011a; Keane et al., 2012; Schöll et al., 2014] on synchronization in delay-coupled neural networks to networks with heterogeneous delays [Cakan et al., 2014]. It shows that the MSF predicts well the behavior of the network if the heterogeneities are sufficiently small but that for larger 
heterogeneities other effects like partial or global amplitude death can - depending on the topology - take place. In [Blaha et al., 2013], synchronization in chemical oscillators was studied where the nodes were slightly non-identical. It turns out that the oscillators synchronize despite the heterogeneity as predicted by the theory of Choe et al. [2010]. In the case that the applied voltage is high such that the oscillations of relaxational type the behavior is well predicted if the theory [Choe et al., 2010] is extended to non-sinusoidal functions.

\subsection{ADAPTIVE CONTROL OF UNCOUPLED SYSTEMS AND NETWORKS}

The MSF is a convenient tool to determine the stability of synchrony in complex networks. In this way it allows for finding parameters which yield a desired behavior - synchronization, desynchronization, or states of group synchrony. However, in real-world applications parameters are often unknown or drift in time. In systems with adaptive control, the control law is chosen such that it automatically finds appropriate control parameters and adapts them to a changing environment. A well known adaptive control is the speed-gradient (SG) method that minimizes a predefined goal function by changing one or several accessible system parameters appropriately [Fradkov, 1979; Fradkov and Pogromsky, 1998; Fradkov, 2005, 2007]. In [Lehnert et al., 2011b], we showed that the SG method can be used for the adaptive control of unstable fixed points and unstable periodic orbits and demonstrated that it is in fact able to cope with drifting system parameters. Furthermore, the SG method has been applied to control cluster states in network motifs [Selivanov et al., 2012; Schöll et al., 2012b,a] and in larger networks [Lehnert et al., 2014].

The control of larger networks considered in [Lehnert et al., 2014] is realized by the adaptation of the network topology. In adaptive networks, the network topology changes according to the dynamical state of the nodes, while, in turn, the state of the nodes is influenced by the topology. The brain is a good example of an adaptive network: The neural dynamics is strongly influenced by the topology, while neural connectivity depends on the spiking behavior of the neurons via synaptic plasticity or via the emergence of new connections. Further examples of adaptive networks are swarm networks, communication networks, the network of blood vessels, social network, food webs, and chemical networks; see [Gross and Blasius, 2008] and references therein. In [Lehnert et al., 2014], the dynamics of the topology is designed such that the control goal, in this case a certain cluster state, is reached. 


\subsection{THE INFLUENCE OF DELAY ON THE DYNAMICS OF NETWORKS}

Throughout this thesis I will consider delayed coupled networks. Delay is a ubiquitous phenomenon in nature and technology and arises whenever time in the propagation or the processing of a signal is needed [Just et al., 2010; Flunkert et al., 2013a]. For example, in laser networks the finite speed of light gives rise to a propagation delay [Lüdge, 2012; Otto, 2013; Soriano et al., 2013]. Time delay in neural networks emanates from the finite speed of the transmission of an action potential between two neurons where the propagation velocity of an action potential varies between 1 to $100 \mathrm{~mm} / \mathrm{ms}$ depending on the diameter of the axon and whether the fibers are myelinated or not [Koch, 1999]. The influence of delay on the dynamics on networks has been investigated by several authors [Kinzel and Kanter, 2008; Kestler et al., 2008; Kinzel et al., 2009; Englert et al., 2010; Zigzag et al., 2010; Flunkert et al., 2010; Rosin et al., 2010; Englert et al., 2011; Kanter et al., 2011b; Heiligenthal et al., 2011; Flunkert et al., 2013b; Dahms, 2011; Popovych et al., 2011; Lücken et al., 2013; Kinzel, 2013; D’Huys et al., 2013; Kantner and Yanchuk, 2013; D’Huys et al., 2014]

Depending on the context, delay can play a constructive or a destructive role. For example, time-delayed feedback control (TDFC) is a well established control method to control unstable periodic orbits embedded in chaotic attractors as well as unstable fixed points [Pyragas, 1992; Ahlborn and Parlitz, 2004; Rosenblum and Pikovsky, 2004; Hövel and Schöll, 2005; Schöll and Schuster, 2008; Grebogi, 2010]. The advantage of TDFC is that it does not rely on a reference system or on an a priori knowledge of the system itself. Furthermore, it is noninvasive for appropriate values of the delay time, meaning that the orbit or the fixed point is not changed by the control. However, the success of the TDFC depends on the choice of appropriate control parameters [Just et al., 1997; Hövel and Schöll, 2005; Dahms et al., 2007]. In [Lehnert et al., 2011b], an adaptive form of TDFC was derived which finds the correct parameter, in this case the coupling strength, in a self-organized way. In [Lehnert et al., 2014], we show that the delay is a crucial parameter shaping the topology of network, which in turn, enables the control of the network.

\subsection{Model Systems}

In this thesis, I mainly consider generic models. As a model for oscillatory systems close to the Hopf bifurcation, I consider the Stuart-Landau oscillator [Kuramoto, 1984]. Neural models or, more general, excitable models can be classified according to their underlying 
bifurcation: Type-I excitability occurs in systems close to a saddle-node infinite period (SNIPER) bifurcation, while type-II arises around a Hopf bifurcation. As a model for typeI, I use the normal form of the SNIPER bifurcation [Hu et al., 1993; Hizanidis et al., 2008]; as an example for type-II, I investigate the FitzHugh-Nagumo system [FitzHugh, 1961; Nagumo et al., 1962].

\subsection{OUTLINE}

This thesis consists of two main parts: In the first part, I consider the stability of zerolag, group, and cluster synchrony in complex networks. The second part explores adaptive control methods with the focus on the control of synchronization patterns in networks.

Before the beginning of part I, Chapter 2 gives an introduction to complex networks. The most important quantities used to characterized the topology of complex networks are introduced. Furthermore, frequently used network models, namely regular, random, small-world, and scale-free networks are explained. The mathematical description of dynamical networks is discussed.

Part I begins with Chapter 3, where an introduction to synchronization and its quantification is given. In particular, a recapitulation of the MSF for zero-lag synchronization is presented.

In Chapter 4, the MSF is applied to networks with excitatory and inhibitory links. Thereby, the two types of excitability are treated separately. It is shown that inhibition in networks of type-II excitability always induces a transition from synchronization to desynchronization, while in networks of type-I excitable elements, depending on the parameters, several transitions between synchronization and desynchronization may take place.

In Chapter 5, the MSF for group and cluster synchrony is developed and applied to networks of SNIPER and FitzHugh-Nagumo systems. It is shown that the MSF as well as the topologies for which group synchrony can be observed are characterized by a discrete rotational symmetry. It is discussed how the restrictions interposed in the derivation of the MSF can be lifted in the case of commuting coupling matrices. Furthermore, a recapitulation of the theory of Choe et al. [2010] on cluster of Stuart-Landau oscillator is given which serves as a basis for later Chapters. The last section of Chapter 5 generalized the MSF for zero-lag as well as for group synchrony to non-smooth systems. 
Part I ends with Chapter 6 where the application of parts of the theory developed in the previous Chapters is investigated in situations where delay or parameter heterogeneities play a role. In the first Section, uni- and bimodal delay distributions in networks of FitzHugh-Nagumo systems are investigated. The second Section treats cluster synchronization in networks of chemical oscillators which are slightly non-identical. Depending on the applied voltage, smooth and relaxational oscillations can be observed where in the second case an extension of the theory is necessary.

Part II starts in Chapter 7 with an introduction to adaptive control concepts with a focus on the SG method. In Chapter 8 an adaptive version of TDFC is developed and applied to an unstable focus and an unstable periodic orbit of the Rössler system. The robustness of the method towards noise, different initial conditions, and drifting parameters is discussed.

In Chapter 9 cluster states in small networks of delay-coupled Stuart-Landau oscillators are controlled adaptively by tuning the phase of the complex coupling strength. To find the adaptive controller, we employ the SG method with an appropriate goal function which is zero in the case of successful control. Advantages and disadvantages of the suggested method are discussed.

In Chapter 10, the control of cluster states in large networks of delay-coupled StuartLandau oscillators is realized via the adaptation of the topology. The topology after control is modulated by the delay time which is discussed with the help of a row-wise discrete Fourier transformation. Furthermore, it shown that the control will also be feasible if only a subset of links is adapted.

The thesis concludes with Chapter 11 where the obtained results are summarized and discussed. Furthermore, an outlook on possible continuations of this work is given. 


\section{2 | COMPlex DyNamical NeTWORKS}

The term complex networks refers to graphs with non-trivial topological features ${ }^{1}$. This Chapter gives a recapitulation on the theory of complex networks, discusses the quantities used to describe networks, and introduces the most important network models namely random [Solomonoff and Rapoport, 1951; Rapoport, 1957; Erdős and Rényi, 1959, 1960], small-world [Watts and Strogatz, 1998; Monasson, 1999], and scale-free networks [Barabasi and Bonabeau, 2003].

A prominent and on the first view surprising feature of most real-world networks is that they are characterized by a small shortest path length which means that it takes only a few steps to get from any node of the network to any other node. This is known as the small-world phenomenon and has been found in such different networks as networks of film actors, brain networks, and the power grid [Watts and Strogatz, 1998]. As also discussed in Chapter 1, further examples include the metabolic network [Jeong et al., 2000], the co-authorship of mathematicians [Barahona and Pecora, 2002], the World Wide Web [Adamic, 1999], food webs [Montoya and Solé, 2002], and the co-occurrence of words (two words are considered as linked if they are neighbors in a sentence) [Ferrer i Cancho and Solé, 2001].

The small-world characteristic is well reflected in an Erdős-Rényi random network, which was the first network model studied in detail and which allows, in many respects, for an analytic treatment. Unfortunately, the model lacks many other features found in real networks. Most prominently, no well connected neighborhoods exist, or, in other words, random networks have a small clustering coefficient, while most real-world networks are characterized by a high clustering coefficient. With the small-world network, [Watts and Strogatz, 1998] presented a model which shows both characteristics; it has a small shortest path lengths, and at the same time a high clustering coefficient.

\footnotetext{
${ }^{1}$ We use the terms graph and network synonymously.
} 
The Chapter is structured as follows: In the first Section, I discuss the mathematical descriptions of networks in terms of the adjacency matrix and the coupling matrix. Section 2.2 introduces the different network quantities used to measure network features. An overview of the different network types is given in Sec. 2.3. In Sec. 2.4 dynamics on networks are discussed.The Chapter is summarized in Sec. 2.4.

\subsection{MATHEMATICAL DESCRIPTION: FROM NODES AND LINKS TO THE COUPLING MATRIX}

A network consists of $N$ nodes and $L$ links connecting these nodes. We distinguish $d i$ rected and undirected networks. In a directed network, a link has a direction, i.e., it points from node $i$ to node $j$, while in an undirected network the coupling is always bidirectional, meaning that for each link from node $i$ to node $j$ another link with the same strength exists which points from node $j$ to node $i$. Synonyms for node and link are vertex and edge, respectively.

A network can be represented by its $N \times N$ adjacency matrix $\mathbf{A}$ which is defined as

$$
A_{i j}=\begin{array}{ll}
1, & \text { if there is a link from node } j \text { to node } i, \\
0, & \text { otherwise. }
\end{array}
$$

The adjacency matrix is very useful for quantifying networks properties. Most of the network quantities discussed in the next Section make use of the adjacency matrix.

The adjacency matrix is a good description for unweighted networks or for problems where weights are negligible. However, real-world networks often have links with different strengths. For example, consider traffic networks, where different roads are used with very different frequencies, or the brain, where neurons are connected via synapses which vary in strength. The different weights are particularly important when it comes to dynamics on networks, the focus of this thesis. In Chapter 10, we will see that changing the weights in a network has a dramatic effect on the dynamics even if the adjacency matrix is kept constant.

In contrast to the adjacency matrix, the coupling matrix takes the different link weights into account: The element $G_{i j}$ of the coupling matrix $\mathbf{G}$ describes a link from node $j$ to node $i$ with weight $G_{i j}$. Note that the entries of $\mathbf{G}$ can be positive and negative. In the context of the brain, this corresponds to excitatory and inhibitory coupling, respectively. 
The adjacency matrix A can be obtained from $\mathbf{G}$ by element-wise use of the Heavisidefunction $\Theta$ :

$$
A_{i j}=\Theta\left(\left|G_{i j}\right|\right),
$$

where $\Theta(x)=1$ if $x>0$, and $\Theta(x)=0$ if $x \leq 0$.

Obviously, adjacency and coupling matrix are symmetric for undirected networks and unsymmetric for directed networks.

\subsection{NETWORK QUANTITIES}

Different aspect of networks can be characterized by a variety of different network quantities [Boccaletti et al., 2006b; Albert and Barabasi, 2002; Newman, 2003; Newman et al., 2006; Newman, 2010]. Here, I briefly introduce the most important quantities and terms to describe a network of $N$ nodes. In parts, the presentation follows [Lehnert, 2010].

Node degree: The degree $\mathscr{D}_{j}$ of node $j$ is defined as the number of links attached to this node. The mean degree $\langle\mathscr{D}\rangle$ is given as the average over all nodes, i.e., $\langle\mathscr{D}\rangle=$ ${ }_{j} \mathscr{D}_{j} / N$. In weighted networks, in- and out-degree are distinguished, where the in-degree counts the number of incoming links and the out-degree the number of outgoing ones.

Degree distribution: The degree distribution $P(\mathscr{D})$ is the probability that a randomly chosen node has $\mathscr{D}$ links. Its first moment is the mean degree $\langle\mathscr{D}\rangle$.

Assortativity: The term assortativity describes the tendency of nodes to connect more often to nodes which are in some way similar than to other nodes. Most often the term is used in the context of degree and is then understood synonymously to degree correlation. The assortativity regarding the degree can be quantified by calculating the average degree of the nearest neighbors of nodes with degree $\mathscr{D}$, denoted as $\mathscr{D}_{n n}(\mathscr{D})$ :

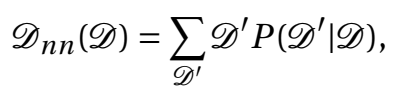

where $P(\mathscr{D} \mid \mathscr{D})$ is the conditional probability that a node of degree $\mathscr{D}$ is linked to a node with degree $\mathscr{D}^{\prime}$. If $\mathscr{D}_{n n}(\mathscr{D})$ increases with $\mathscr{D}$ the network is assortative, otherwise we will call it disassortative. In the case that $\mathscr{D}_{n n}(\mathscr{D})$ is independent of $\mathscr{D}$ no degree correlations exist. 
Community structure: A community is a subgraph whose nodes are more tightly connected to each other than to the rest of the network. Social networks often exhibit a community structure. A variety of different approaches have been developed to define and detect communities; for a review see [Fortunato, 2010].

Component: A network is considered as connected if a path exists between every node pair $i$ and $j$. Otherwise it will be referred to as disconnected. A disconnected network is composed of several components where the components are the largest possible, connected subgraphs. A connected network consists of one single component comprising all nodes. In this thesis, I will only consider connected networks.

In Erdős-Rényi random networks, a critical connection probability $q_{c}=1 / N$ exists above which a giant component emerges, meaning that almost all nodes are connected within one component and only a few are part of other components. For details see Subsec. 2.3.2.

Shortest path length: $\mathscr{L}$ is the shortest distance between two nodes, averaged over all nodes:

$$
\mathscr{L}=\frac{1}{N^{2}}{ }_{i, j} g_{i j}
$$

$g_{i j}$ is the shortest distance (or geodesic distance) between node $i$ and node $j$, defined as the minimal number of links one has to traverse to get from node $i$ to node $j$.

Note that definition (2.4) includes the diagonal terms $g_{i i}$. In a bidirectional network without self-coupling, $g_{i}$ is equal to 2: One step is needed to reach a nearest neighbor, the second one to get back to the original node. Thus, including the diagonal terms in the definition (2.4) contributes a constant term of $2 / N$ in networks without self-coupling. With self-coupling, $g_{i i}=1$ since a link exists which starts and ends at the $i$ th node. As a consequence, the shortest path length is reduced in networks including self-coupling compared to ones without self-coupling.

Disconnected networks call for another definition since $g_{i j}$ is infinite if node $i$ and $j$ are not in the same component. A definition using the reciprocal values of the geodesic distances reads

$$
\mathscr{L}^{-1}=\frac{1}{N^{2}} g_{i, j}^{-1} .
$$


Clustering coefficient: In real-world networks, two nodes connected to the same node are often more likely to be linked than two arbitrary nodes. As an example, consider a social network. Friends of a given person are much more likely to be mutual friends than two randomly picked people. The clustering coefficient $\mathscr{C}$ allows for quantifying this phenomenon. A high clustering coefficient means that with a higher probability a link exists between nodes that are adjacent to the same nodes than between two randomly picked nodes.

Formally, $\mathscr{C}$ is defined as follows: Let $\ell_{i}$ denote the number of links between the neighbors of the $i$ th node. Then the clustering coefficient of the $i$ th node is defined as

$$
\mathscr{C}_{i}=\frac{2 \ell_{i}}{\mathscr{D}_{i}\left(\mathscr{D}_{i}-1\right)},
$$

where $\mathscr{D}_{i}$ is the degree of the $i$ th node, i.e., the number of neighbors node $i$ has. Thus, the clustering coefficient is defined as the ratio between the actual existing links $\ell_{i}$ and the maximum possible number of links $\mathscr{D}_{i}\left(\mathscr{D}_{i}-1\right) / 2$ between the neighbors of the $i$ th node. The clustering coefficient of the network is obtained by averaging over all nodes:

$$
\mathscr{C}=\frac{1}{N} \mathscr{C}_{i}
$$

Betweenness: The betweenness measures the number of shortest paths going through a given node. Thus, the betweenness is helpful for quantifying how important a node is for the communication in a social network or transport in a railway network, for example.

The betweenness of the $i$ th node is defined as

$$
\mathscr{B}_{i}=\frac{n_{j k}(i)}{n_{j k}},
$$

where $n_{j k}$ is the number of all shortest paths $g_{j k}$ between the $j$ th and the $k$ th node, and $n_{j k}(i)$ is the number of these shortest paths which pass through the $i$ th node.

Closeness: The closeness $\mu_{i}$ of node $i$ is the inverse of the average distance of the $i$ th node from all other nodes:

$$
\mu_{i}=\frac{N}{{ }_{j} g_{i j}},
$$

where $g_{i j}$ is the shortest distance between node $i$ and $j$. 
Spectral density: The spectral density of the adjacency matrix A or the coupling matrix G of dimension $N$ is given by

$$
\rho(v)=\frac{1}{N}{ }_{j=0}^{N-1} \delta\left(v-v_{j}\right)
$$

where $v_{j}, j=0, \ldots, N-1$, are the eigenvalues of $\mathbf{A}$ or $\mathbf{G}$, respectively. In the limit of $N \rightarrow \infty$, $\rho(v)$ becomes a continuous function.

\subsection{NETWORK TOPOLOGIES}

At first glance, real-world networks often appear to be completely in random structure. However, most are characterized by distinct topological features e.g., they might have a particularly high clustering coefficient, a small shortest path-length, or might be characterized by the existence of a few hubs, e.g., nodes of a particularly high degree. Several models including random, small-world, and scale-free networks have been developed which reflect these topological features. Furthermore, ideal network structures which allow for calculating the eigenvalue spectrum analytically have been considered. In Chapter 3, the importance of the eigenvalue spectrum for the synchronizability of a network will be discussed. The current Section introduces different topologies, their constructing methods and discusses their eigenvalue spectrum.

\subsubsection{Regular networks with circulant matrices}

An $N \times N$ circulant matrix $\mathbf{C}$ is given by the condition $C_{i j}=c_{(j-i+N)} \bmod N, i, j=0, \ldots, N-$ 1. Thus, $\mathbf{C}$ can be written as

$$
\mathbf{C}=\begin{array}{ccccc}
c_{0} & c_{1} & c_{2} & \cdots & c_{N-1} \\
c_{N-1} & c_{0} & c_{1} & \cdots & c_{N-2} \\
\vdots & \vdots & \vdots & \ddots & \vdots \\
c_{2} & c_{3} & c_{4} & \cdots & c_{1} \\
c_{1} & c_{2} & c_{3} & \cdots & c_{0}
\end{array} .
$$


Circulant matrices are of particular interest as their eigenvalues and eigenvectors are analytically accessible. The eigenvectors are given by [Gray, 2005]

$$
\mathbf{e}_{j}=\frac{1}{\sqrt{N}}\left(1, \omega_{j}, \omega_{j}^{2}, \ldots, \omega_{j}^{n-1}\right)^{T}, \quad j=0, \ldots, N-1,
$$

where $\omega_{j}=\exp \frac{2 \pi \mathrm{i} j}{N}$ is the $N$ th roots of unity. The corresponding eigenvalues can be calculated as

$$
v_{j}={ }_{k=0}^{N-1} c_{k} \omega_{j}^{k}
$$

A subclass of networks with circulant matrices are regular ring networks. In a regular ring network, each node is with equal strength connected to its $k$ nearest neighbors to the right and to the left. Thus, the normalized coupling matrix is given by

$$
C_{i j}=\frac{1}{2 k} \begin{array}{lll}
1, & \text { if }|(i-j+N) \bmod N| \leq k \text { and } i \neq j
\end{array}
$$

With Eq. (2.13), the eigenvalues can be calculated as

$$
v_{j}=\frac{1}{k}_{l=1}^{k} \cos \frac{2 \pi j l}{N} .
$$

Figure 2.1 (a) shows a regular ring network with $k=2$, i.e., nearest and next-nearest neighbors are linked. (Panel (b) and (c) will be explained in Secs. 2.3.3 and 2.3.2, respectively.) Regular ring are of particular interest as they exhibit chimera states under certain conditions [Kuramoto and Battogtokh, 2002; Abrams and Strogatz, 2004; Sethia et al., 2008; Wolfrum and Omel'chenko, 2011; Wolfrum et al., 2011; Omelchenko et al., 2011, 2012; Hagerstrom et al., 2012; Omelchenko et al., 2013; Vüllings et al., 2014; Zakharova et al., 2014; Omelchenko et al., 2015]. In a chimera state, the nodes in the network separate into two groups with distinctly different behavior, e.g., spatially coherent and incoherent behavior or coherent and incoherent oscillations.

The unidirectional ring network is another special case of a circulant matrix network. It 
(a)

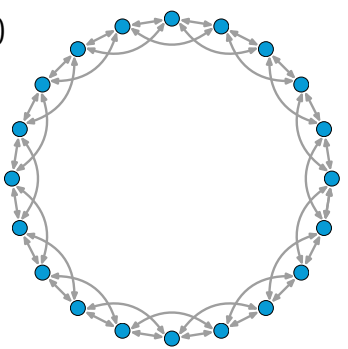

(b)

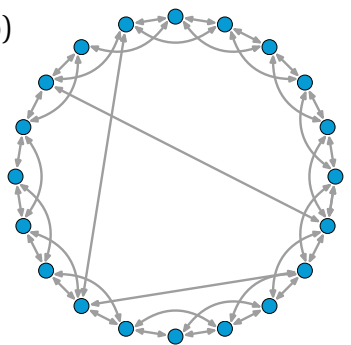

(c)

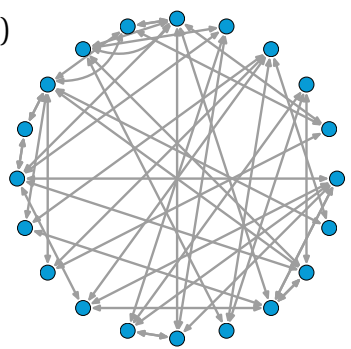

Figure 2.1.: Rewiring procedure in the Watts-Strogatz model. (a) $p=0$ (regular network). (b) $p=0.08$ (small-world network). (c) $p=1$ (random network). Parameters: $N=20$, $k=2$. Figure modified from [Lehnert, 2010].

is given by the coupling matrix

$$
\mathbf{C =} \begin{array}{ccccc}
0 & 1 & 0 & \cdots & 0 \\
0 & 0 & 1 & \cdots & 0 \\
\vdots & \vdots & \vdots & \ddots & \vdots \\
0 & 0 & 0 & \cdots & 1 \\
1 & 0 & 0 & \cdots & 0
\end{array}
$$

and its eigenvalues read

$$
v_{j}=e^{\frac{2 \pi \mathrm{i}}{N} j}, \quad j=0, \ldots, N-1
$$

Another important example of a circulant matrix network is the all-to-all topology, i.e., a network where each node is connected to all other nodes. If we normalize each row to one, i.e., $\quad{ }_{j} C_{i j}=1, j=0, \ldots, N-1, \mathbf{C}$ is described by $C_{i j}=1 /(N-1)$ for $i \neq j$ assuming that we exclude self-coupling. A normalization to one is useful when considering dynamics on networks, as will be discussed in Sec. 3.1. The eigenvalues are given by

$$
v_{j}=\begin{array}{ll}
0, & \text { if } j=0 \\
\frac{-1}{N-1}, & \text { if } j>0 .
\end{array}
$$




\subsubsection{Erdős-Rényi random networks}

Random networks have been among the first to be studied in network science. They were independently proposed by Rapoport [1957], Solomonoff and Rapoport [1951], and most prominently by Erdős and Rényi [1959, 1960]. Two different possibilities to construct a random network exist:

- In a directed (undirected) graph, $N^{2}(N(N-1) / 2)$ links can exist. In the first model, $L(N)$ links are randomly distributed on these possible positions [Erdős and Rényi, 1959].

- In the second model, each of the possible position is occupied with the probability $q$. The expectation value of the number of links is then $q N^{2}$ (directed network) or $q N(N-1) / 2$ (undirected network) [Erdős and Rényi, 1960].

Obviously, in the limit of large $N$ and $L$, both construction methods coincide.

In the following discussion, a directed, unweighted network is assumed. For such a network, Erdős and Rényi $[1959,1960]$ showed that a critical probability $q_{c}=1 / N$ exists separating the following regimes:

$\mathbf{q}<\mathbf{q}_{\mathbf{c}}$ : For $N \rightarrow \infty$, with probability one the graph has no component larger than $\mathscr{O}(\ln N)$, where $\mathscr{O}$ denotes "of the order of".

$\mathbf{q}=\mathbf{q}_{\mathbf{c}}:$ The largest component has the size $\mathscr{O}\left(N^{2 / 3}\right)$.

$\mathbf{q}>\mathbf{q}_{\mathbf{c}}$ : A giant component of size $\mathscr{O}(N)$ arises and no other component greater than $\mathscr{O}(\ln N)$ exists.

For $q>q_{c}$ and large $N$, the spectral density $\rho(v)$ of an undirected graph converges to

$$
\rho(v)=\begin{array}{ll}
\frac{\sqrt{4 N q(1-q)-v^{2}}}{2 \pi N q(1-q)}, & \text { if }|v|<2 \overline{N q(q-1)}, \\
0, & \text { otherwise. }
\end{array}
$$

Equation (2.19) is know as Wigner's law or semicircle law [Wigner, 1958]. The largest eigenvalue $v_{0}$ is separated from the bulk and increases with the network size as $p N$.

At the beginning of this Chapter, I have discussed the small-world phenomenon, i.e, the fact that many real-world networks are characterized by a small shortest path length. More formally, we speak of the small-world effect if the shortest path length of a network grows slower than $\log (N)$, i.e., $\mathscr{L}(N) \leq \alpha \log (N)$ with $\alpha \in \mathbb{R}$. The shortest path length of a random network is given by $\mathscr{L}=\log N / \log \mathscr{D}$ where $\mathscr{D}=L(N) / N=q N$ is the mean 
degree [Newman, 2003]. Obviously, this fulfills the criteria of the small-world effect if we increase the network size while keeping the average degree fixed. However, in most other aspects the random network is a poor model for real-world networks. Its clustering coefficient is given by $\mathscr{C}=q$ which tends to $1 / N$ if we let $N$ go to infinity for constant $\mathscr{D}$, while real-world network often exhibit high clustering coefficients. Furthermore, in contrast to real-world networks, the random network has a Poissonian degree distribution but no community structure, and no assortativity [Newman, 2003].

\subsubsection{Small-world networks}

The shortcoming of the random network to reflect real-world network properties motivated Watts and Strogatz [1998] to their seminal work on small-world networks. The small-world network is characterized by both, a small shortest path length and a high clustering coefficient.

The Watt-Strogatz model relies on a one-dimensional interpolation between a regular network and a random network as it is depicted in Fig. 2.1. This interpolation involves the following steps (for details see [Watts and Strogatz, 1998]):

1. Start with a regular network of $N$ nodes where every node is linked to its $2 k$ neighbors (see Sec. 2.3.1 and Fig. 2.1(a)). Watts and Strogatz required $N \gg k \gg \ln (N) \gg 1$ ensuring that the regular ring is sparse but remains connected during the following procedure.

2. Then, rewire one end of each link with probability $p$ to a new node, where duplicated links are forbidden; see Fig. 2.1(b).

In the limiting cases of $p=0$ and $p=1$, this procedure recovers the regular ring and the random network, respectively; for a schematic view see Fig. 2.1(a) and (c), respectively.

More interesting is the case of intermediate $p$. In this regime, which is also called smallworld regime, the regular ring is still undamaged in large parts, while a few long-range connections exist; see Fig. 2.1(b). Watts and Strogatz [1998] showed that this configuration yields a high clustering coefficient and a small shortest path length. Figure 2.2 shows the normalized shortest path length $\mathscr{L} / \mathscr{L}(p=0)$ (blue symbols) and the normalized clustering coefficient $\mathscr{C} / \mathscr{C}(p=0)$ (red symbols) as a function of the rewiring probability $p$. The small-world regimes, where $\mathscr{L}$ is small and $\mathscr{C}$ is large, is approximately located between $p=0.001$ and $p=0.01$. 


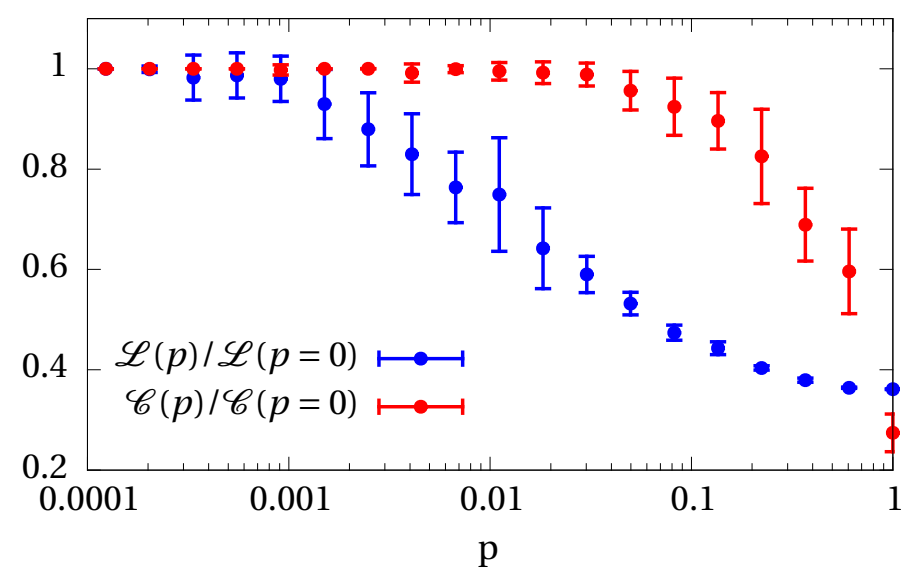

Figure 2.2.: Small-world network. Shortest path length $\mathscr{L} / \mathscr{L}(p=0)$ (blue symbols) and clustering coefficient $\mathscr{C} / \mathscr{C}(p=0)$ (red symbols) versus rewiring probability $p$. Parameters: $N=100, k=4$. Number of realizations: 70. (Reproduction of Fig. 2 in [Watts and Strogatz, 1998])

\subsubsection{Scale-free networks}

In a scale-free network, the degree distribution $P(\mathscr{D})$ follows a power law, $P(\mathscr{D}) \sim \mathscr{D}^{-\beta}$, $\beta>0$, for large $\mathscr{D}$, where $\mathscr{D}$ is the degree of a given node. This implies the existence of hubs: Nodes that have a very large number of links attached to them, while the majority of nodes have only a few links. These hubs are of great importance for the behavior of the whole network. For example, a scale-free network is very sensitive to the failure of a hub.

Famous examples of scale-free networks include the World Wide Web [Albert and Barabasi, 2002], citation networks [de Solla Price, 1965], the collaboration of mathematicians [Grossman and Ion, 1995], the Internet at the level of autonomous systems [Chen et al., 2002], and the interaction network of proteins [Jeong et al., 2001].

\subsection{DYNAMICS ON NETWORKS}

So far I considered the structure of networks, i.e., how the nodes in a network are connected via links. In many applications, the structure is not sufficient to describe the complexity of the system but it has to be taken into account that the nodes are dynamical 
systems interacting through links. This approach has been used in very different fields. For example, a node can represent a laser which interacts via its light output with other lasers [Dahms, 2011; Flunkert, 2011]. In epidemiology, SIR models are used to describe the spread of diseases: The nodes are individuals which can be susceptible (S), infected (I), or recovered (R). The links correspond to contacts between the individuals [Anderson and May, 1992; Hethcote, 2000; Belik et al., 2011; Lentz et al., 2012]. Neural networks gained a lot of attention in the last decades. Here, the node dynamics are described by neural models and the links represents the interaction of the neurons via action potentials; see [Ernst et al., 1998; Dayan and Abbott, 2005; Timme et al., 2006; Jahnke et al., 2008; Vogels and Abbott, 2009; Hövel, 2010; Vogels et al., 2011; Popovych et al., 2011, 2013] and many more. Furthermore, many authors investigated the interplay between dynamical nodes and networks structure on a fundamental level in particular with a focus on synchronization [Pecora and Carroll, 1998; Chavez et al., 2006; Arenas et al., 2006b; Boccaletti et al., 2006a; Hunt et al., 2010; Flunkert et al., 2010; Sorrentino, 2014; Geffert, 2015].

For all examples mentioned above, the dynamics on a network can be described as a set of coupled differential equations, $i=0, \ldots, N-1$,

$$
\dot{\mathbf{x}}_{i}=\mathbf{f}_{i}\left[\mathbf{x}_{i}(t)\right]+\sigma{ }_{j=0}^{N-1} G_{i j} \mathbf{h}_{\mathbf{i j}}\left(\mathbf{x}_{i}, \mathbf{x}_{j}\right),
$$

where $\mathbf{x}_{i} \in \mathbb{C}^{d}$ is the state of the $i$ th node and $\mathbf{f}_{i}$ the local dynamics which describes the uncoupled node. $\sigma=K \exp (\mathrm{i} \beta), K, \beta \in \mathbb{R}$, is the complex coupling strength. The function $\mathbf{h}_{i j}$ models the coupling between node $i$ and node $j$. $\mathbf{G}$ is the coupling matrix as introduced in Sec. 2.3. For simplicity, we assumed that the states of all nodes are of dimension $d$. From Sec. 3.1 it will be clear that this is one of several necessary conditions for the existence of a zero-lag synchrony solution. However, group synchrony can exist in networks where the nodes are not of the same dimension as will be discussed in Chapter 5 .

Often and throughout this thesis it is assumed that the coupling is linear and diffusive. In this case, Eq. (2.20) reads

$$
\dot{\mathbf{x}}_{i}=\mathbf{f}_{i}\left[\mathbf{x}_{i}(t)\right]+\sigma{ }_{j=0}^{N-1} G_{i j} \mathbf{H}_{i j}\left(\mathbf{x}_{j}-\mathbf{x}_{i}\right)
$$

where $\mathbf{H}_{i j}$ is a $d \times d$ matrix called the coupling scheme. 


\subsubsection{Delayed coupling}

As discussed in Chapter 1, delay is a ubiquitous phenomenon in real-world systems. Two types of delay can be distinguished: Propagation and processing delays. Here we consider the first type, i.e., a time delay which arises due to the finite propagation speed between node $i$ and $j$. Including this delay, Eq. (2.21) becomes

$$
\dot{\mathbf{x}}_{i}=\mathbf{f}_{i}\left[\mathbf{x}_{i}(t)\right]+\sigma{ }_{j=0}^{N-1} G_{i j} \mathbf{H}_{i j} \mathbf{x}_{j}\left(t-\tau_{i j}\right)-\mathbf{x}_{i}(t), \quad i=0, \ldots, N-1 .
$$

Equation (2.21) is a delay differential equation (DDE). DDEs are mathematically challenging since their phase spaces are infinite. In order to find a solution of Eq. (2.22) for $t>0$ the value of $\mathbf{X} \equiv\left(\mathbf{x}_{0}, \ldots, \mathbf{x}_{N-1}\right)$ has to be known on the interval [ $\left.-\tau_{\max }, 0\right]$ where $\tau_{\max }$ is the maximum time delay, i.e., $\tau_{\max }=\max _{i j} \tau_{i j}$. In other words, we have to specify the initial value or history function

$$
\mathbf{X}(s)=\Phi(s), \quad s \in\left[-\tau_{\max }, 0\right] .
$$

This is in contrast to ordinary differential equation where defining $\mathbf{X}(0)$ is sufficient.

Only in rare cases, DDEs are analytically solvable by employing the methods of steps in which case the DDE is integrated stepwise over an interval of length $\tau_{\text {max }}$, i.e., for step $j$ over the interval $\left[j \tau_{\max },(j+1) \tau_{\max }\right]$, where $j=0, \ldots$ [Fridman, 2014]. Linear DDEs can be solved semi-analytically by considering the roots of their characteristic equation (see Appendix A). However, in most cases DDEs have to be solved numerically. Details can be found in [Farmer, 1982]. For reviews on time delayed systems see [Erneux, 2009; Atay, 2010; Fridman, 2014].

\subsection{SUMMARY}

This Chapter has given an introduction to networks. I have discussed the mathematical representation of networks and different network quantities. Network types and their eigenvalue spectra have been addressed. The development of these network types has been driven by the observations that many real networks from very different areas exhibit similar characteristics. In particular, they often have a high clustering coefficient and a small shortest path length. These features are very well reflected by the smallworld model by Watts and Strogatz [1998]. This model uses a rewiring procedure to interpolate between a regular network and random graph. For intermediate rewiring rates 
- the small world regime - the model displays a high clustering coefficient and a small shortest paths length.

Besides describing the topology of real-world networks, networks can be used to model the dynamics and interactions of many systems or agents. In this case, the network is represented by a set of coupled ordinary differential equations or, if delay in the coupling is considered, by a set of delay differential equations. The aim of this thesis is to explore the interplay between the topology of networks and the dynamics on the network. Furthermore, in Chapter 9 it will be shown how the topology can be changed in targeted manner to control the dynamics on the network. A particularly interesting dynamical state on a network is when all nodes follow the same dynamics or, in other word, are synchronized. In the next Chapter, this state will be discussed in detail. 
Part I

\section{STABILITY OF SYNCHRONIZATION}





\section{SYNCHRONIZATION IN COMPLEX NETWORKS}

In the previous Chapter, complex dynamical networks have been introduced. One particularly interesting dynamical state on a network is synchronization because synchrony phenomena are ubiquitous in nature and engineering, as discussed in detail in Chapter 1 . The stability analysis of synchrony in a complex network is a challenging problem due to the high dimensionality of the equations. The master stability function (MSF) is a convenient tool to address this problem as it allows for decomposing the high dimensional problem to $N$ problems of lower dimensions where $N$ is the number of nodes in the network [Pecora and Carroll, 1998; Pecora, 1998; Fink et al., 2000].

The aim of this Chapter is to discuss synchronization and, in particular, the MSF and its role in determining the stability of synchrony. In Sec. 3.1, I will introduce the notion of synchronization and explain the conditions imposed on a network such that a synchronized solution exists. Section 3.2 is a recapitulation of the MSF as suggested by Pecora and Carroll [1998]. In Sec. 3.3, the Kuramoto order parameter is investigated as a convenient tool to evaluate the quality of synchronization. The Chapter is summarized in Sec. 3.4.

Note that I only study zero-lag synchronization in this Chapter; group and cluster synchrony are the topics of Chapter 5. I will consider the case of delay-coupled networks since they are in the focus of this thesis. The simplification to networks without delay as, for example, originally considered by Pecora and Carroll, is straightforward when setting the delay time equal to zero. 


\subsection{SYNCHRONIZATION}

In the following, I discuss which conditions the dynamical equation of a network and the topology have to fulfill such that a synchronized solution exists. Recall the dynamical equation of a network of $N$ nodes with time-delayed coupling (see Eq. (2.22)):

$$
\dot{\mathbf{x}}_{i}=\mathbf{f}_{i}\left[\mathbf{x}_{i}(t)\right]+\sigma{ }_{j=0}^{N-1} G_{i j} \mathbf{H}_{i j} \mathbf{x}_{j}\left(t-\tau_{i j}\right)-\mathbf{x}_{i}(t), \quad i=0, \ldots, N-1,
$$

where $\mathbf{x}_{i} \in \mathbb{C}^{d}$ is the state of node $i$ and $\mathbf{f}_{i}$ its local dynamics. $\sigma=K \exp (\mathrm{i} \beta), K, \beta \in \mathbb{R}$, is the complex coupling strength, $\mathbf{G}$ is the coupling matrix, $\tau_{i j}$ and $\mathbf{H}_{i j}$ are the time delay and the coupling scheme between nodes $j$ and $i$, respectively.

In the case of zero-lag synchronization (also called isochronous or inphase synchrony) all nodes follow the same trajectory without a time shift

$$
\mathbf{x}_{0}(t)=\ldots=\mathbf{x}_{N-1}(t) \equiv \mathbf{x}_{s}(t) .
$$

Equation (3.2) consists of $d(N-1)$ constraints and, thus, defines a $d$-dimensional hyperplane in the $d N$-dimensional phase space of Eq. (3.1). In the following, I synonymously refer to this hyperplane as synchronization manifold or synchronized solution. Inserting condition (3.2) into Eq. (3.1) yields

$$
\dot{\mathbf{x}}_{s}=\mathbf{f}_{i}\left[\mathbf{x}_{s}(t)\right]+\sigma{ }_{j=0}^{N-1} G_{i j} \mathbf{H}_{i j} \mathbf{x}_{s}\left(t-\tau_{i j}\right)-\mathbf{x}_{s}(t), \quad i=0, \ldots, N-1 .
$$

A necessary condition for the existence of a synchronous solution is that the input to all nodes is identical in the synchronous case. For Eq. (3.3), this means that the right hand side has to be independent of $i$. Obviously, this is only fulfilled if $\mathbf{f}_{i} \equiv \mathbf{f}, \mathbf{H}_{i j} \equiv$ $\mathbf{H}, \tau_{i j} \equiv \tau$, and ${ }_{j=0}^{N-1} G_{i j}=g_{i} \equiv g$ for all $i, j=0, \ldots, N-1$, where $g$ is the row sum of G.

Substituting this into Eq. (3.1) yields

$$
\dot{\mathbf{x}}_{i}=\mathbf{f}[\mathbf{x}(t)]+\sigma \underset{j=0}{N-1} G_{i j} \mathbf{H} \mathbf{x}_{j}(t-\tau)-\mathbf{x}_{i}(t), \quad i=0, \ldots, N-1,
$$

the form of the network I will consider in most parts of this thesis. Equation (3.3) de- 
scribing the evolution of the synchronous state reads

$$
\dot{\mathbf{x}}_{s}=\mathbf{f}\left[\mathbf{x}_{s}(t)\right]+g \sigma \mathbf{H}\left[\mathbf{x}_{s}(t-\tau)-\mathbf{x}_{s}(t)\right] .
$$

Obviously, each row sum $g \neq 0$ can be rescaled by the absolute value $K$ of the coupling strength $\sigma=K \exp (i \beta)$. Thus, without loss of generality we can assume $g=1$. We conclude that a synchronous solution of Eq. (3.3) only exists if

1. All nodes follow the same dynamics if uncoupled $\left(\mathbf{f}_{i} \equiv \mathbf{f}\right)$.

2. The coupling between all node pairs has to be identical $\left(\mathbf{H}_{i j} \equiv \mathbf{H}\right)$.

3. All propagation delays between nodes have to be the same $\left(\tau_{i j} \equiv \tau\right)$.

4. The row sum of the coupling matrix $\mathbf{G}$ is equal for all rows ( $\underset{j=0}{N-1} G_{i j}=g_{i} \equiv g$ ).

In the following, I refer to condition (iv) as the equal-row-sum condition or, in the case $g=1$, as the unity-row-sum condition. Only in Sec. 5.3 are networks with $g \neq 1$ considered as the case $g=0$ is investigated.

\subsection{MASTER STABILITY FUNCTION}

In the previous Section, the prerequisites for the existence of a synchronous solution were discussed. In the following the stability of this solution is investigated with the help of the MSF as introduced by Pecora and Carroll [1998].

Stability of synchronization can be evaluated in a straightforward manner, through a linearization of the network dynamics at the synchronous state. From the resulting variational equation, the stability can then be determined by, for example, calculating the largest Lyapunov exponent $\Lambda$. The idea of the MSF is to calculate $\Lambda$ not directly from the variational equation for a given network topology, but to perform a diagonalization of the variational equation, afterwards calculating $\Lambda$ as a function of the generalized complex eigenvalues $v$ of the coupling matrix. Thus, the MSF allows for separating the effects of the local dynamics from the effects of the topology. The clear advantage is that $\Lambda(v)$ has to be computed only once and can then be used to check the stability of any given coupling matrix. This is not only an advantage in terms of numerical costs but allows for drawing general conclusions by systematically analyzing eigenspectra of networks. For example, in Chapter 4 the eigenspectra of small-world and random networks with inhibitory links will be investigated with the help of the MSF. An introduction to the eigenspectra of the most important network models was given in Chapter 2. 


\subsubsection{Derivation of the master stability function}

In the following, the MSF is derived for a delay-coupled network [Dhamala et al., 2004; Choe et al., 2010; Dahms, 2011; Kinzel et al., 2009; Heiligenthal et al., 2011; Kinzel, 2013; D'Huys et al., 2013]. If the delay time is set to zero, the results of [Pecora and Carroll, 1998] for a network without delayed coupling are recovered.

Equation (3.1) can be written in a compact way by defining $\mathbf{X} \equiv\left[\mathbf{x}_{0}, \mathbf{x}_{1}, \ldots, \mathbf{x}_{N-1}\right]$ and $\hat{\mathbf{f}}(\mathbf{X}) \equiv\left[\mathbf{f}\left(\mathbf{x}_{0}\right), \mathbf{f}\left(\mathbf{x}_{1}\right), \ldots, \mathbf{f}\left(\mathbf{x}_{N-1}\right)\right]:$

$$
\dot{\mathbf{X}}(t)=\hat{\mathbf{f}}[\mathbf{X}(t)]-\sigma \mathbb{1}_{N} \otimes \mathbf{H X}(t)+\sigma[\mathbf{G} \otimes \mathbf{H}] \mathbf{X}(t-\tau),
$$

where $\otimes$ denotes the Kronecker product. The properties of the Kronecker product needed in this Chapter are described in Appendix B.

A linearization of Eq. (3.6) at the synchronous solution, given by Eq. (3.5), yields the variational equation

$$
\delta \dot{\mathbf{X}}(t)=\left.D \hat{\mathbf{f}}\right|_{\mathbf{x}_{s}(t)} \delta \mathbf{X}(t)-\sigma \mathbb{1}_{N} \otimes \mathbf{H} \delta \mathbf{X}(t)+\sigma(\mathbf{G} \otimes \mathbf{H}) \delta \mathbf{X}(t-\tau),
$$

where $\left.D \hat{\mathbf{f}}\right|_{\mathbf{x}_{s}(t)}$ is the Jacobian of $\hat{\mathbf{f}}$ which has to be evaluated at the synchronization manifold $\mathbf{x}_{s}$. The evolution of $\mathbf{x}_{s}$ is given by Eq. (3.5). Equation (3.7) describes the time evolution of an initially small perturbation from the synchronous state: $\delta \mathbf{X}(t) \equiv \mathbf{X}(t)-\mathbf{X}_{s}(t)$, where $\mathbf{X}_{s}(t)=\left[\mathbf{x}_{s}, \mathbf{x}_{s}, \ldots, \mathbf{x}_{s}\right]$. The first term in Eq. (3.7) has a block form since the local dynamics of each node only depends on its own state. Thus, the term can be rewritten as $\left.D \hat{\mathbf{f}}\right|_{\mathbf{x}_{s}(t)}=\left.\mathbb{1}_{N} \otimes D \mathbf{f}\right|_{\mathbf{x}_{s}(t)}$ where $\left.D \mathbf{f}\right|_{\mathbf{x}_{s}(t)}$ is the Jacobian of $\mathbf{f}$. The block form of this term is not affected if we now diagonalize $\mathbf{G}$. The aim of this block diagonalization is to separate the directions transversal to the synchronization manifold from the directions within this manifold. In the following, the diagonalization, which is the key step of the derivation in the MSF, is explained.

Let $\mathbf{S}$ be the matrix diagonalizing $\mathbf{G}$, i.e., $\mathbf{D}_{G}=\mathbf{S G} \mathbf{S}^{-1}$, where $\mathbf{D}_{G}$ is the diagonal form of $\mathbf{G}$. We block diagonalize Eq. (3.7) by multiplying it with $\mathbf{S} \otimes \mathbb{1}_{d}$ from the left, where $\mathbb{1}_{d}$ is the $d$ - dimensional unity matrix:

$$
\left(\mathbf{S} \otimes \mathbb{1}_{m}\right) \delta \dot{\mathbf{X}}(t)=\left(\mathbf{S} \otimes \mathbb{1}_{m}\right)\left[\mathbb{1}_{N} \otimes\left(\left.D \mathbf{f}\right|_{\mathbf{x}_{s}(t)}-\sigma \mathbf{H}\right)\right] \delta \mathbf{X}(t)+\sigma\left(\mathbf{S} \otimes \mathbb{1}_{m}\right)(\mathbf{G} \otimes \mathbf{H}) \delta \mathbf{X}(t-\tau) .
$$

Using the rules for matrix multiplication (see Appendix B), Eq. (3.8) is rewritten as

$$
\left(\mathbf{S} \otimes \mathbb{1}_{m}\right) \delta \dot{\mathbf{X}}(t)=\mathbb{1}_{N} \otimes\left(\left.D \mathbf{f}\right|_{\mathbf{x}_{s}(t)}-\sigma \mathbf{H}\right)\left(\mathbf{S} \otimes \mathbb{1}_{m}\right) \delta \mathbf{X}(t)+\sigma(\mathbf{S G}) \otimes\left(\mathbb{1}_{m} \mathbf{H}\right) \delta \mathbf{X}(t-\tau) .
$$


Diagonalizing $\mathbf{G}$ and using fact that $\mathbb{1}_{m}$ commutes with all other matrices yields

$$
\left(\mathbf{S} \otimes \mathbb{1}_{m}\right) \delta \dot{\mathbf{X}}(t)=\mathbb{1}_{N} \otimes\left(\left.D \mathbf{f}\right|_{\mathbf{x}_{s}(t)}-\sigma \mathbf{H}\right)\left(\mathbf{S} \otimes \mathbb{1}_{m}\right) \delta \mathbf{X}(t)+\sigma\left(\mathbf{D}_{G} \mathbf{S}\right) \otimes\left(\mathbf{H} \mathbb{1}_{m}\right) \delta \mathbf{X}(t-\tau) .
$$

We rearrange the second term on the right hand side according to the rules for the Kronecker product given in Appendix B:

$$
\left(\mathbf{S} \otimes \mathbb{1}_{m}\right) \delta \dot{\mathbf{X}}(t)=\mathbb{1}_{N} \otimes\left(\left.D \mathbf{f}\right|_{\mathbf{x}_{s}(t)}-\sigma \mathbf{H}\right)\left(\mathbf{S} \otimes \mathbb{1}_{m}\right) \delta \mathbf{X}(t)+\sigma\left(\mathbf{D}_{G} \otimes \mathbf{H}\right)\left(\mathbf{S} \otimes \mathbb{1}_{m}\right) \delta \mathbf{X}(t-\tau) .
$$

By introducing $\delta \tilde{\mathbf{X}}=\left(\mathbf{S} \otimes \mathbb{1}_{m}\right) \delta \mathbf{X}$, Eq. (3.11) can be rewritten as

$$
\delta \dot{\tilde{\mathbf{X}}}(t)=\mathbb{1}_{N} \otimes\left(\left.D \mathbf{f}\right|_{\mathbf{x}_{s}(t)}-\sigma \mathbf{H}\right) \delta \tilde{\mathbf{X}}(t)+\sigma\left(\mathbf{D}_{G} \otimes \mathbf{H}\right)\left(\mathbf{S} \otimes \mathbb{1}_{m}\right) \delta \tilde{\mathbf{X}}(t-\tau) .
$$

Now, both terms on the right hand side have a block structure:

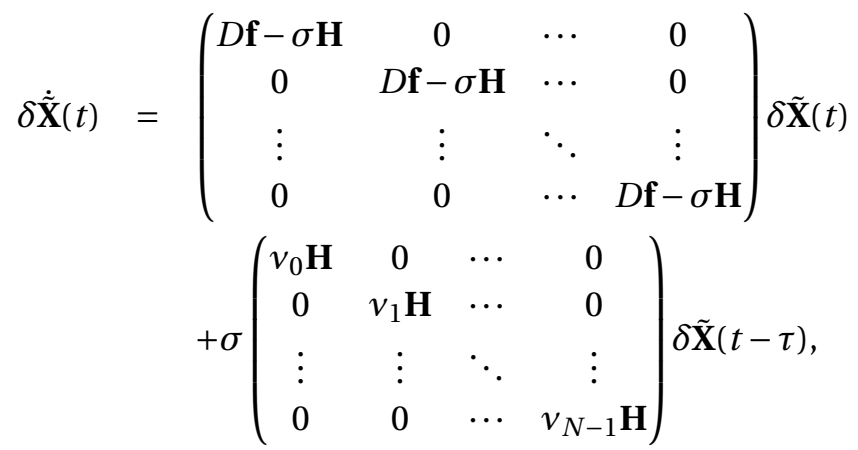

where $\left\{v_{i}\right\}_{i=0, \ldots, N-1}$ are the eigenvalues of G. Equation (3.13) is equivalent to $N$ blocks of the form

$$
\delta \dot{\tilde{\mathbf{x}}}_{i}(t)=\left(\left.D \mathbf{f}\right|_{\mathbf{x}_{s}(t)}-\sigma \mathbf{H}\right) \delta \tilde{\mathbf{x}}_{i}(t)+\sigma v_{i} \delta \tilde{\mathbf{x}}_{i}(t-\tau), \quad i=0, \ldots, N-1 .
$$

where $\left\{\delta \tilde{\mathbf{x}}_{i}\right\}_{i=0, \ldots, N-1}$ represents the $m$-dimensional perturbations from the synchronous state in the eigensystem of G. From Eq. (3.14) the stability of synchronization in a network can be evaluated by calculating the Lyapunov exponent $\Lambda\left(v_{i}\right)$ for each value of $v_{i}$, $i=0, \ldots, N-1 . \quad v_{0}$ is called the longitudinal eigenvalue and will be discussed in Subsec. 3.2.3. $v_{i}, i=1, \ldots, N-1$, are the transversal eigenvalues, the corresponding Lyapunov exponents determine the stability of synchronization. If the largest of these $N-1$ Lyapunov exponents $\Lambda\left(v_{i}\right)$ is negative synchronization, in the network will be stable. Otherwise it will be unstable.

Alternatively to computing the Lyapunov exponents for each specific network topology, 
Pecora and Carroll suggested to compute $\Lambda$ as function of the generalized eigenvalues $v$ and then evaluate $\Lambda(\operatorname{Re} v, \operatorname{Im} v)$ at the $N$ values of $v_{i}$. The advantage is, as discussed above, that $\Lambda(\operatorname{Re} v, \operatorname{Im} v)$ has to be calculated only one time and can then be used to study the stability of any given network. The generalized form of Eq. (3.14) with the $v$ instead of $v_{i}, i=0, \ldots, N-1$, is called the master stability equation (MSE) and reads

$$
\delta \dot{\tilde{\mathbf{x}}}(t)=\left(\left.D \mathbf{f}\right|_{\mathbf{x}_{s}(t)}-\sigma \mathbf{H}\right) \delta \tilde{\mathbf{x}}(t)+\sigma v \delta \tilde{\mathbf{x}}(t-\tau),
$$

The largest Lyapunov exponent $\Lambda(v)$ as a function of the generalized eigenvalue $v$ constitutes the MSF.

\subsubsection{The longitudinal eigenvalue}

As discussed above a necessary condition for the existence of a synchronous solution is that the row sum of the coupling matrix is equal to 1 (unity-row-sum condition). If this condition is fulfilled, one eigenvalue equal to 1 exists which is the eigenvalue $v_{0}$ to the eigenvector $\mathbf{v}_{0}=(1,1, \ldots, 1)$, which can easily seen by multiplying $\mathbf{G}$ by $\mathbf{v}_{0}$ :

$$
\mathbf{G v}_{0}=G_{j} G_{0 j}, \ldots,{ }_{j} G_{N-1, j}{ }^{T}=(1, \ldots, 1)^{T}=v_{0} \mathbf{v}_{0},
$$

where we made use of unity-row-sum condition, i.e., ${ }_{j} G_{i j}=1, i=0, \ldots, N-1$, in the second step.

The eigenvalue $v_{0}$ is called the longitudinal eigenvalue as it describes perturbation longitudinal to the synchronization manifold because for $v_{0}=1$, the master stability equation (3.15) coincides with the variational equation of the synchronous dynamics given in Eq. (3.2). The other eigenvalues are referred to as transversal eigenvalues since they described perturbations transversal to the synchronization manifold.

The longitudinal Lyapunov exponent $\Lambda\left(v_{0}\right)$, i.e., the Lyapunov exponent evaluated at the longitudinal eigenvalue, describes the dynamics within the synchronization manifold. If its value is negative, the synchronous dynamics is given by a fixed point. $\Lambda\left(v_{0}\right)=0$ indicates that the synchronization manifold is a limit cycle and is the Lyapunov exponent which corresponds to the Goldstone mode of this limit cycle. A positive longitudinal Lyapunov exponent means that the synchronous dynamics is chaotic. Table 3.1 summarizes the possible dynamics within the synchronization manifold. Note that this only applies when calculating the MSF numerically. If an analytic calculation of the MSF is possible, 


\begin{tabular}{cc}
\hline Longitudinal Lyapunov exponent $\Lambda\left(v_{0}\right)$ & Type of synchronous dynamics \\
\hline$\Lambda\left(v_{0}\right)<0$ & fixed point \\
$\Lambda\left(v_{0}\right)=0$ & limit cycle \\
$\Lambda\left(v_{0}\right)>0$ & chaotic \\
\hline
\end{tabular}

Table 3.1.: Dynamics within the synchronization manifold as determined by the longitudinal Lyapunov exponent $\Lambda\left(v_{0}\right)$ when numerically calculating the MSF.

$\Lambda\left(v_{0}\right)>0$ can also be observed for fixed points or limit cycles, namely, in the case that the synchronous dynamics is an unstable solution of Eq. (3.5).

\subsubsection{Symmetry of the MSF for zero-lag synchrony}

If we consider real-valued node dynamics and a real-valued coupling strength, the MSF for zero-lag synchrony is symmetric with respect to the $\operatorname{Re} v$-axis, i.e., $\Lambda(v)=\Lambda\left(v^{*}\right)$, where * denotes the complex conjugate of a variable. This can be shown by applying the transformation $v \rightarrow v^{*}$ to Eq. (3.15) which yields

$$
\delta \dot{\tilde{\mathbf{x}}}(t)=\left(\left.D \mathbf{f}\right|_{\mathbf{x}_{s}(t)}-K \mathbf{H}\right) \delta \tilde{\mathbf{x}}(t)+K v^{*} \delta \tilde{\mathbf{x}}(t-\tau),
$$

where $\sigma=K$ because we required $\sigma$ to be real. Furthermore, we assume that $\left.D \mathbf{f}\right|_{\mathbf{x}_{s}(t)}, \mathbf{H} \epsilon$ $\mathbb{R}$. This is the case for all models considered in this thesis but the Stuart-Landau oscillator. Note that nevertheless $\delta \mathbf{x} \in \mathbb{C}$ because $v$ is a complex parameter. Then, the complex conjugate of Eq. (3.17) reads

$$
\delta \dot{\tilde{\mathbf{x}}}^{*}(t)=\left(\left.D \mathbf{f}\right|_{\mathbf{x}_{s}(t)}-K \mathbf{H}\right) \delta \tilde{\mathbf{x}}^{*}(t)+K v \delta \tilde{\mathbf{x}}^{*}(t-\tau) .
$$

If we now apply the transformation $\delta \tilde{\mathbf{x}}^{*} \rightarrow \delta \tilde{\mathbf{x}}$, we recover Eq. (3.15). Consequently, if Eq. (3.15) has a solution $\delta \tilde{\mathbf{x}}(t)$, Eq. (3.17) has a solution $\delta \tilde{\mathbf{x}}^{*}(t)$. Since $|\delta \tilde{\mathbf{x}}(t)|=\left|\delta \tilde{\mathbf{x}}^{*}(t)\right|$ the corresponding Lyapunov exponents have to be equal, i.e., $\Lambda(v)=\Lambda\left(v^{*}\right)$. As an example consider the MSF for the saddle-node infinite period (SNIPER) bifurcation model shown in Fig. 3.1. The SNIPER model and its MSF will be introduced in detail in Chapter 4; here it serves as an illustration of the symmetry of the MSF with respect to the $\operatorname{Re} v$-axis. 


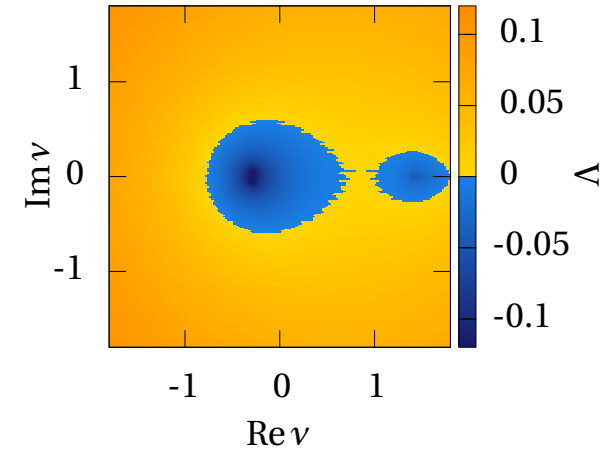

\subsection{QUANTIFYING SYNCHRONIZATION}

In networks with heterogeneities in the nodes or in the delays, a perfectly synchronized solution does not exist because the conditions for synchrony discussed in Sec. 3.1 are not fulfilled. However, states close to synchrony may exist. To measure the degree of synchronization of these states in oscillatory systems, the Kuramoto order parameter can be used [Kuramoto, 1984]:

$$
R=\frac{1}{N_{s}}{ }_{j=0}^{N_{s}-1} e^{\mathrm{i} \varphi_{j}}
$$

$\varphi_{j}$ is the phase of the $j$ th oscillator and can be defined as

$$
\varphi_{j}=2 \pi \frac{t-t_{n}}{t_{n}-t_{n-1}}
$$

where $t_{n}$ is the time of the previous spike of the $j$ th neurons [Rosenblum et al., 2001]. For $R=1$, perfect phase synchronization is reached; if $R \approx 0$, the network is desynchronized. $N_{s}$ in Eq. (3.19) is the number of spiking nodes. In other words, we do not include nonspiking nodes when calculating the Kuramoto order parameter. This will become important in Chapter 6.1 where networks with heterogenous coupling delays are studied. It will be shown that partial or global amplitude death is induced when the heterogeneities become too large. A generalization of the Kuramoto order parameter to cluster states is discussed in Chapter 9. 


\subsection{SUMMARY}

In this Chapter, an introduction to the topic of synchronization in complex networks has been given and the conditions for the existence of a synchronous solution have been discussed in detail. The stability of the synchronous solution can be accessed via the master stability function (MSF). The MSF allows for separating the directions transversal to the synchronization manifold from the ones longitudinal to it. Then, the stability of a network can be determined by considering the Lyapunov exponents associated with the transversal directions. If these exponents are all negative, the synchronization is stable; otherwise it is unstable.

Furthermore, in this Chapter I have introduced the Kuramoto order parameter as a convenient measure of the degree of synchrony. This will be of particular interest in Chapter 6, where heterogenous delay distributions will be considered which can result in nearly synchronized states. These states will be characterized with the help of the Kuramoto order parameter. 


\section{4 | CONTROL OF SYNCHRONIZATION TRANSITIONS BY BALANCING EXCITATORY AND INHIBITORY COUPLING}

Determining the stability of synchronization in large and complex networks is very challenging due to the high dimensionality of the problem. In the previous Chapter, I have given an introduction to the master stability function (MSF) which allows for decoupling of the topology and the local dynamics. In this way the originally very high-dimensional problem is reduced to (i) solving the problem for one node with a rescaled coupling strength and (ii) determining the eigenvalues of the network's coupling matrix.

In this Chapter, I want to discuss the application of the master stability function to neural networks. Neurons are well described by excitable systems, where type-I and type-II excitability can be distinguished [Hodgkin, 1948; Rinzel and Ermentrout, 1989; Izhikevich, 2000a; Lindner et al., 2004]. However, excitability is not limited to neural systems but was also observed in a wide range of natural and engineering systems including the cardiovascular systems [Yang and Wang, 2008], chemical systems like the BelousovZhabotinskii reaction [Mikhailov et al., 1994], optoelectronical oscillators [Rosin et al., 2011], Boolean networks realized by logic gates [Rosin et al., 2012], laser systems [Wünsche et al., 2001; Goulding et al., 2007; Barbay et al., 2011], and glacial ocean oscillations [Ganopolski and Rahmstorf, 2002]. For a review on neural excitability see [Izhikevich, 2000a].

The aim of this Chapter is to study the type-I and type-II excitability on two generic models, the saddle-node infinite period (SNIPER) bifurcation model, also known as the SNIC (saddle-node bifurcation on invariant cycle) model [Hu et al., 1993; Hizanidis et al., 2008], and the FitzHugh-Nagumo model [FitzHugh, 1961; Nagumo et al., 1962] In Sec. 4.1, I explain the notion of excitability and the differences between the two types. Furthermore, the models are introduced and the MSF for both models is presented. In Sec. 4.2, 
the eigenvalues of small-world networks with excitatory and inhibitory coupling are calculated and compared with the MSFs. This comparison shows that inhibition, depending on the type of excitability and the delay time, introduces one or multiple transitions between synchronization and desynchronization.

\subsection{Stability OF EXCITABle SySTEMS}

In this Section, the notion of excitability is discussed. Furthermore, the two different types of excitability are introduced and the MSFs calculated for the example models, the SNIPER model and the FitzHugh-Nagumo model, are presented and discussed.

\subsubsection{Excitability}

An excitable system is a system with a stable fixed point which responds to perturbations as follows: Small (subthreshold) perturbations decay rapidly, while large (superthreshold) perturbations trigger a strongly non-linear response such that the system undergoes a large excursion in the phase space before returning to the fixed point. In the case of a neural system this perturbation mimics an action potential. During the time that the system needs to return to the fixed point, no new spike can be released (or only under very large perturbations); this time is called the refractory period and is typical for neural systems [Izhikevich, 2000b; Dayan and Abbott, 2005].

Excitable behavior is characteristic for systems close to a bifurcation to the oscillatory regime. Often the phase space changes only locally when the bifurcation parameter changes such that the system undergoes a bifurcation from the oscillatory regime to the regime with the stable fixed point, i.e., the excitable regime. A remnant of the limit cycle sometimes called "ghost" remains which the system follows after superthreshold bifurcations causing the excursion in the phase space. The two types of excitability are classified according to the type of bifurcation.

\subsubsection{Type-I excitability}

Type-I excitability arises close to a saddle-node infinite period (SNIPER) bifurcation, also known as the SNIC bifurcation (saddle-node bifurcation on invariant cycle). At the bifurcation point, this bifurcation and, thus, type-I excitability in general are characterized by the emergence of a limit cycle with infinite period, i.e., zero frequency, but non vanishing 

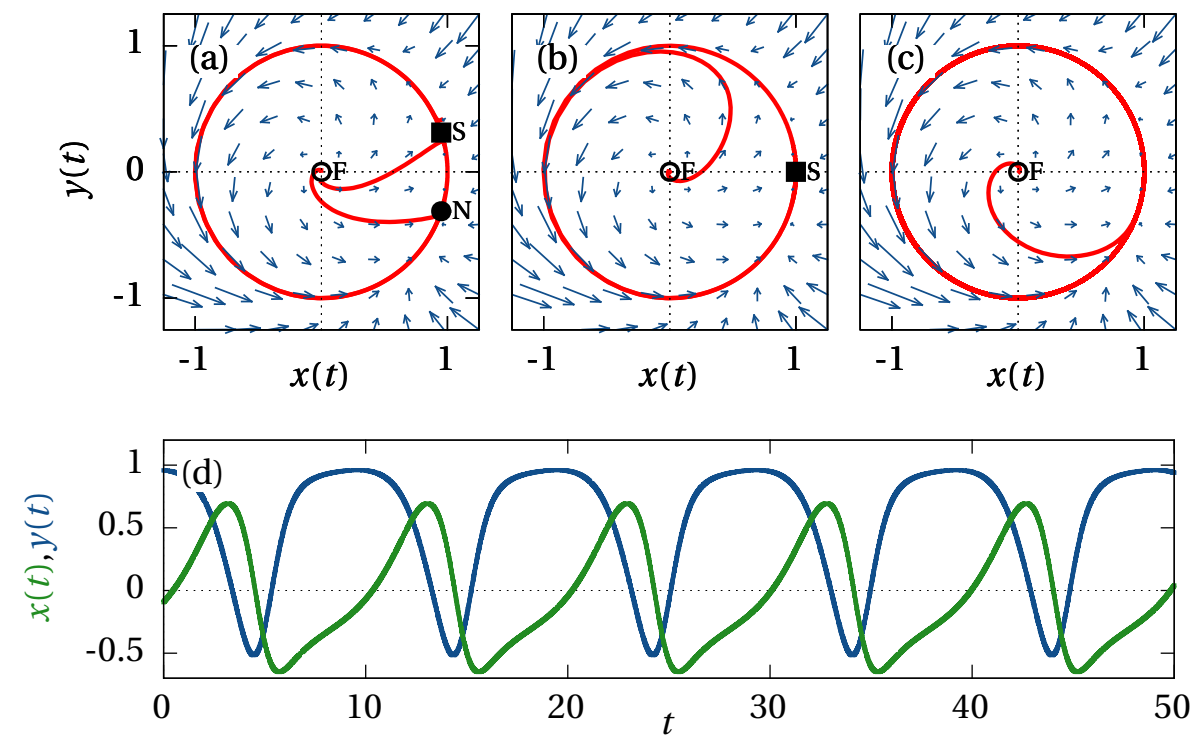

Figure 4.1.: SNIPER model. Phase portraits and typical trajectories of the uncoupled system (4.1): (a) below the SNIPER bifurcation, $b=0.95$; (b) at the bifurcation, $b=1$; and (c) above the bifurcation, $b=1.05$. Labels: F: unstable focus; S: Saddle; $N$ : stable node. (d) Time series of the coupled system (4.4) with $\tau=8, \sigma=0.4$, and $b=0.95$. The green line shows $x(t)$, the blue one $y(t)$.

amplitude. Examples of models showing type-I excitability are the Ermentrout-Kopell canonical model also known as theta model [Ermentrout and Kopell, 1986], the Connor model for crab leg axons [Connor et al., 1977], for certain parameters the Morris-Lecar model [Morris and Lecar, 1981], the Wang-Buzsaki model for inhibitory interneurons [Wang and Buzsáki, 1996], and the Hindmarsh-Rose model [Hindmarsh and Rose, 1982, 1984]. Near the bifurcation point, all these models can be described by the dynamics of a SNIPER normal form.

Hence, this normal form of the SNIPER bifurcation will be investigated [Hu et al., 1993; Hizanidis et al., 2008], mathematically represented by two first-order differential equations:

$$
\mathbf{f}(\mathbf{x})={ }_{\dot{y}}^{\dot{x}}=\begin{aligned}
& x\left(1-x^{2}-y^{2}\right)+y(x-b) \\
& y\left(1-x^{2}-y^{2}\right)-x(x-b)
\end{aligned},
$$

where $b \in \mathbb{R}$ is the bifurcation parameter. 
In polar coordinates $(x=r \cos \varphi, y=r \sin \varphi)$, Eq. (4.1) reads

$$
\begin{aligned}
\dot{r} & =r 1-r^{2}, \\
\dot{\varphi} & =b-r \cos \varphi .
\end{aligned}
$$

At $b=1$, the SNIPER bifurcation takes place. Figure 4.1 shows the phase portraits and typical trajectories of the uncoupled system: (a) below the SNIPER bifurcation, i.e., $b=$ 0.95 , (b) at the bifurcation, i.e., $b=1$, and (c) above the bifurcation, i.e., $b=1.05$. For $b<$ 1 , three fixed points exist: an unstable focus $(\mathrm{F})$ at $(0,0)$, a stable node $(\mathrm{N})$ at $\left(b,-\sqrt{1-b^{2}}\right)$, and a saddle (S) at $\left(b, \sqrt{1-b^{2}}\right)$. In this regime, no stable limit cycle exists but the trajectories approach the stable node (or the saddle, if the initial conditions are chosen directly on its stable manifold which is, however, of measure zero). At $b=1$, the saddle and the node collide and annihilate. At this point a limit cycle with infinite period is born, which is, as discussed above, characteristic for this type of bifurcation. $b>1$ corresponds to the oscillatory regime, where the period $T$ of the limit cycle takes a finite value. Close to the bifurcation, $T$ follows a characteristic scaling law: $T \propto(b-1)^{-1 / 2}$ [Hizanidis et al., 2008].

$b<1$ corresponds to the excitable regime. Without perturbations the system remains in the stable node. Under small perturbations it immediately returns to the node. However, if a perturbation is large enough to push the system beyond the saddle it has to follow the remnant of the limit cycle before returning to the fixed point and a spiking behavior can be observed.

\subsubsection{Type-I excitability : The master stability function}

Oscillations in the excitable regime cannot only be introduced by perturbations but also by time-delayed coupling. In [Keane et al., 2012], a system of $N$ nodes (labeled $i=$ $0, \ldots, N-1$ ) was considered

$$
\dot{\mathbf{x}}_{i}=\mathbf{f}\left(\mathbf{x}_{i}\right)+K{ }_{j=0}^{N-1} G_{i j} \mathbf{H}\left(\mathbf{x}_{j}(t-\tau)-\mathbf{x}_{i}(t)\right)
$$

where $\mathbf{f}\left(\mathbf{x}_{i}\right)$ is the local dynamics of each element $\mathbf{x}_{i}=\left(x_{i}, y_{i}\right)$ as described by Eq. (4.1). $\mathbf{G}$ is the coupling matrix (see Sec. 2.1), and $\mathbf{H}$ is the coupling scheme determining which variables couple. $\mathbf{H}$ is taken to be the $2 \times 2$ identity matrix; this means that the $x$ variable of node $j$ at time $t-\tau$ is coupled to the $x$ variable of node $i$ at time $t$ and analogously for the $y$ variable but that there is no cross-coupling between the $x$ and $y$ variables. The 
coupling parameters, which are identical for all connections, are the real-valued coupling strength $K$ and the delay time $\tau$.

According to Eq. (3.15), the master stability equation (MSE) reads

$$
\delta \dot{\mathbf{x}}(t)=\left(\left.D \mathbf{f}\right|_{\mathbf{x}_{s}(t)}-K \mathbf{H}\right) \delta \mathbf{x}(t)+K v \delta \mathbf{x}(t-\tau),
$$

where the synchronized dynamics $\mathbf{x}_{s}(t)$ is given by (see also Eq. (3.2))

$$
\dot{\mathbf{x}}_{s}=\mathbf{f}\left[\mathbf{x}_{s}(t)\right]+K \mathbf{H}\left[\mathbf{x}_{s}(t-\tau)-\mathbf{x}_{s}(t)\right],
$$

and the Jacobian matrix of the local dynamics reads

$$
\left.D \mathbf{f}\right|_{\mathbf{x}_{s}(t)}=\begin{array}{cc}
1-3 x^{2}-y(y-1) & x-2 x y-b \\
-2 x(y+1)+b & 1-x^{2}-3 y^{2}
\end{array} .
$$

Recall that $v$ is the continuous form of the eigenvalues of $\mathbf{G}$ and is in general a complex number.

In [Keane et al., 2012], the MSF was calculated for different delay times. Figure 4.2 shows the results for (a) $\tau=6$, (b) $\tau=6.5$, (c) $\tau=7$, and (d) $\tau=10$. Obviously, the stable region of the MSF coincides with the unit circle for large delay as can be seen in panel (d). Such behavior is typical for oscillatory systems and large delays. In the limit of infinite delay, this has been proven[Flunkert et al., 2010]. With decreasing delay, the cycle is deformed and finally splits into two separate islands.

Figure 4.3 further explores the influence of the delay time on the stability region. It shows the largest Lyapunov exponent as a function of the real part $\operatorname{Re} v$ of the eigenvalues and the delay $\tau$ for vanishing imaginary part $(\operatorname{Im} v=0)$, and fixed coupling strengths of (a) $K=0.25$, (b) $K=0.3$, and (c) $K=0.35$. In all three panels, at least three different regions can be identified: For large $\tau$, the stability region coincides with the interval $\operatorname{Re} v \in[-1,1]$ (as expected from the results of Flunkert et al. [2010]), for intermediate $\tau$ the stability region becomes smaller or larger than this interval but remains connected, while it splits into two or more disconnected islands below a critical delay time $\tau_{c}$. In panel (a) and (c), a fourth regime, where $\Lambda<0$ for all values of $\operatorname{Re} v$ and fixed $\tau$, is visible for very small delays. In this regime the synchronous dynamics corresponds to fixed point dynamics which, however, is not the focus of this study.

Figures 4.2 and 4.3 demonstrate that the delay time is a crucial parameter for the synchronization properties of the SNIPER model. The substantially different forms of the MSF for large and small delay will result in very different synchronization-desynchronization behavior as will be discussed in Sec. 4.2. 

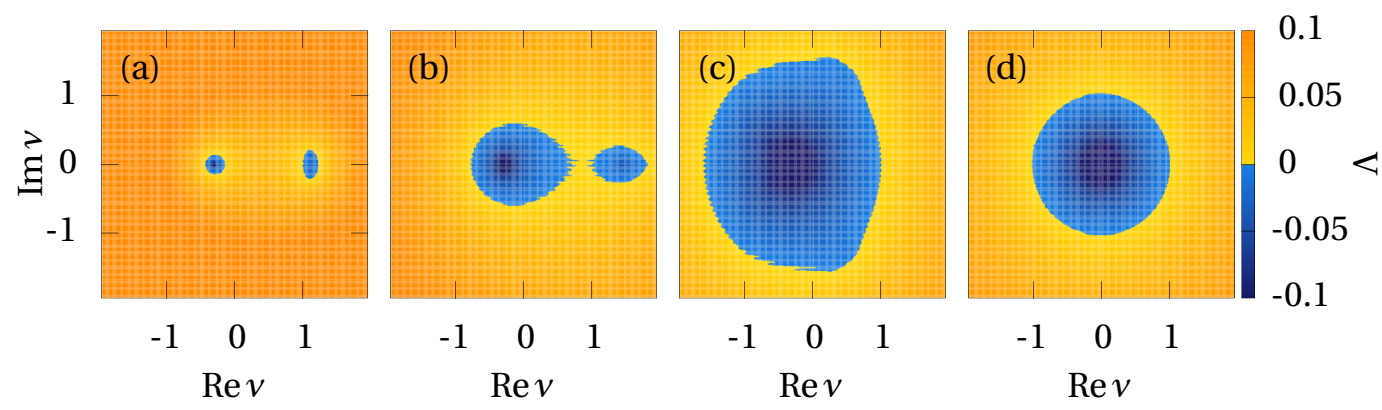

Figure 4.2.: Master stability function (MSF) for the SNIPER model. Largest Lyapunov exponent $\Lambda$ for different delay times: (a) $\tau=6$, (b) $\tau=6.5$, (c) $\tau=7$, and (d) $\tau=10 . v$ is the eigenvalue of the coupling matrix, and $\Lambda$ is the largest Lyapunov exponent. Other parameters: $K=0.35, b=0.95$. Figure reproduced from [Keane et al., 2012].
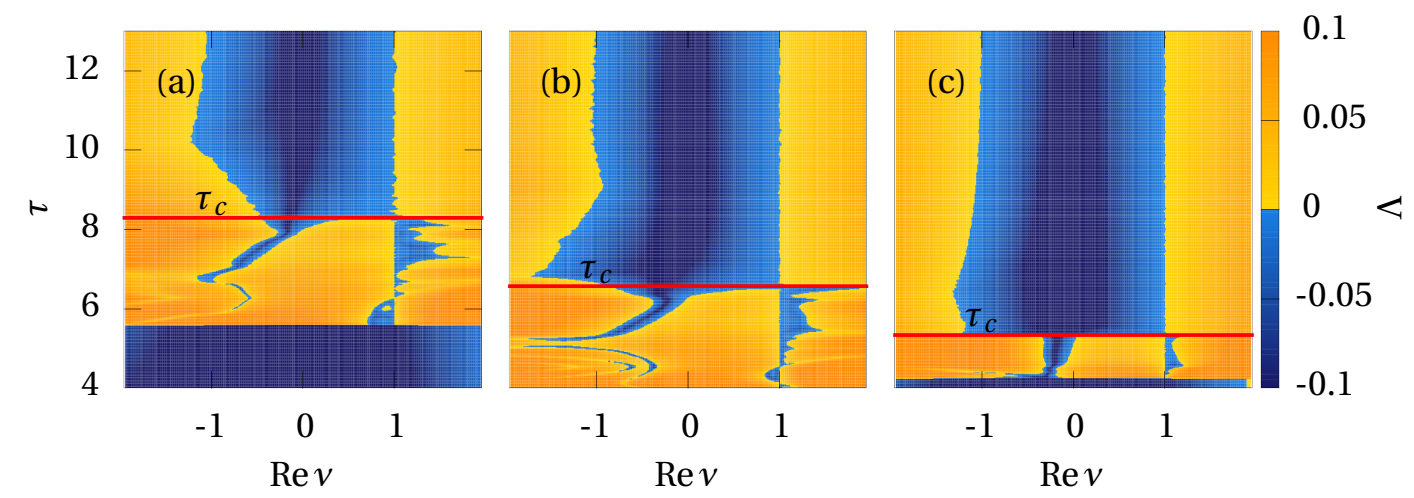

Figure 4.3.: Master stability function (MSF) $\Lambda$ for a fixed coupling strength of (a) $K=0.25$, (b) $K=0.3$ and (c) $K=0.35$ in the plane of the real part $\operatorname{Re} v(\operatorname{Im} v=0)$ and the delay time $\tau$. The horizontal red lines show the position of the critical delay time $\tau_{c} . b=$ 0.95. Figure reproduced from [Keane et al., 2012]. 


\subsubsection{Type-II excitability}

Type-II excitability arises near a Hopf-bifurcation. Close to the bifurcation the amplitude is arbitrarily low but the frequency is finite. Examples of type-II excitability include the FitzHugh-Nagumo system [FitzHugh, 1961; Nagumo et al., 1962], Hodgkin-Huxley model [Hodgkin, 1948; Hodgkin and Huxley, 1952], and for certain parameters the Morris-Lecar model [Morris and Lecar, 1981].

Here, the focus is on the FitzHugh-Nagumo system. The equation for a single system without coupling reads

$$
\begin{aligned}
\epsilon \dot{u} & =u-\frac{u^{3}}{3}-v \\
\dot{v} & =u+a
\end{aligned}
$$

where $u$ is called the activator, and $v$ the inhibitor. $a$ is a threshold parameter whose role will be discussed below. $\epsilon \ll 1$ is a time-scale parameter; in this thesis $\epsilon=0.01$. Consequently, $u$ is a fast variable, while $v$ is slow. The interplay between a fast and a slow variable enables the emergence of a spike. Though the FitzHugh-Nagumo is not a biological model in the sense that it allows for a quantitative description of neural processes, it qualitatively reproduces the spiking behavior of a neuron. $u$ resembles the voltage rise at a neuron, while $v$ summarizes all inhibitory processes taking place during the course of an action potential.

The system has a fixed point at $\left(u^{*}, v^{*}\right)=\left(-a,-a+a^{3} / 3\right)$, which is stable for $a>1$ and unstable for $a<1$. At $a=1$, the supercritical Hopf bifurcation takes place. Thus, $a$ is the bifurcation parameter determining whether the system is excitable $(a>1)$ or exhibits self-sustained periodic firing $(a<1)$. Figure 4.4 shows (a) the phase space, and (b) the time series of a FitzHugh-Nagumo system in the excitable regime after a perturbation sufficiently large to perturb the system from its stable fixed point A. After a large excursion in phase space corresponding to a spike, the system slowly returns to the fixed point (trajectory from D to A). This part of the trajectory resembles the refractory period found in neural systems. For a detailed discussion see [Lehnert, 2010, Chapter 2].

\subsubsection{Type-II excitability: The master stability function}

As done in Sec. 4.1.3 for the SNIPER model, this Section discusses the MSF of the FitzHughNagumo model. The same form of delayed coupling is used. Thus the network of $N$ 


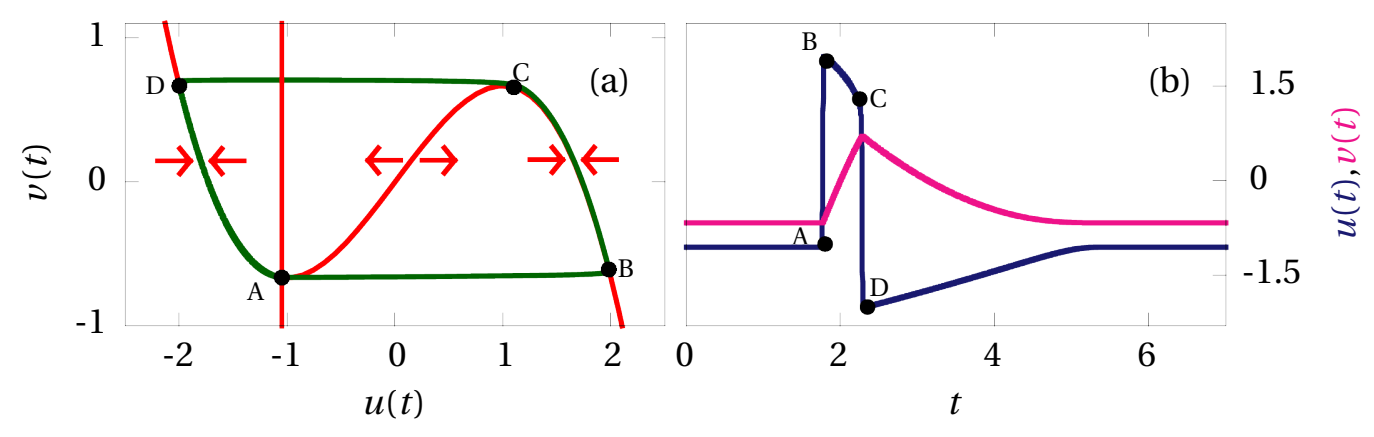

Figure 4.4.: Dynamics of a FitzHugh-Nagumo system in the excitable regime after being sufficiently perturbed from the stable fixed point A. (a) Phase space of the FitzHughNagumo system. Dark green line: trajectory, red lines: nullclines. (b) Time series of a spike. Dark blue line: activator $u$, pink line: inhibitor $v$. A, B, C, D mark corresponding points in panels (a) and (b). Red arrows: Schematic depiction of the phase portrait close to the cubic nullcline. Parameters: $a=1.05, \epsilon=0.01$. Figure reproduced from [Schöll et al., 2009].

nodes is described by

$$
\dot{\mathbf{x}}_{i}=\mathbf{f}\left(\mathbf{x}_{i}\right)+K{ }_{j=0}^{N-1} G_{i j} \mathbf{H}\left(\mathbf{x}_{j}(t-\tau)-\mathbf{x}_{i}(t)\right),
$$

where $\mathbf{x}_{i}=\left(u_{i}, v_{i}\right)$, and the local dynamics $\mathbf{f}\left(\mathbf{x}_{i}\right)$ is given by Eq. (4.8). The coupling scheme $\mathbf{H}$ reads

$$
\mathbf{H}=\begin{array}{cc}
1 / \epsilon & 0 \\
0 & 0
\end{array} .
$$

Meaning that the delayed $u$ variable of node $j$ is coupled into the $u$ variable of node $i$.

Straightforwardly, the MSE is given by Eq. (4.5) with the synchronized dynamics described by Eq. (4.6), and $\mathbf{f}$ given by Eq. (4.8). The Jacobian matrix $D \mathbf{f}$ evaluated at the synchronization manifold $\mathbf{x}_{s}=\left(u_{s}, v_{s}\right)$ reads

$$
\left.D \mathbf{f}\right|_{\mathbf{x}_{s}(t)}=\begin{array}{cc}
\left(1-u_{s}^{2}\right) / \epsilon & -1 / \epsilon \\
1 & 0
\end{array} .
$$




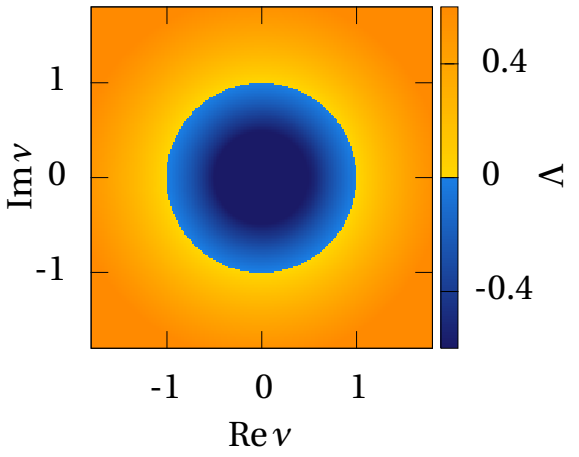

Figure 4.5.: MSF for the FitzHugh-Nagumo system as given by Eq. (3.15) with Eqs. (3.2) and (4.11). $K=0.3$ and $\tau=1$. Figure modified from [Lehnert et al., 2011a].

In [Lehnert, 2010; Lehnert et al., 2011a], it was shown that the MSF for all values of $K$ and $\tau$ shows qualitatively the same behavior. In particular, the stable region is in very good approximation given by the unit cycle. The only prerequisite is that $K$ and $\tau$ have to be sufficiently large to induce oscillations as a synchronous solution. Exemplarily, Fig. 4.5 shows the MSF for the FitzHugh-Nagumo model for $K=0.3$ and $\tau=$ 1.

The period $T$ of the synchronous oscillations is given by $T=\tau+\delta$ with $\delta \ll \tau$ accounting for a short activation time [Schöll et al., 2009; Dahlem et al., 2009]. Thus, the delay time is of the same order as the period. Though Flunkert et al. [2010] predicted that the stable region coincides with the unit circle only in the limit of infinite delay, practically their results often hold in good approximation if the delay is of the same order of magnitude as the characteristic time scale of the underlying system. Thus, the exceptional independence of the stable region from the coupling parameter $K$ and $\tau$ in the FitzHugh-Nagumo system might be explainable by the delay time setting a period which is of the same order of magnitude as the delay.

\subsection{SYNCHRONIZATION-DESYNCHRONIZATION TRANSITIONS}

This Section discusses the stability of synchronization in networks with a mix of inhibitory and excitatory links, in particular, small-world and random networks are discussed. An introduction to the network model is given in Subsec. 4.2.1. The previous Section suggested that - depending on the model and the delay time - two different classes of MSFs arise when evaluating the synchronization properties of networks:

A For the FitzHugh-Nagumo system, i.e., type-II excitability, and, for large delay, for the SNIPER model, i.e., type-I excitability, the stable region of the MSF coincides 
with the unit circle. The impact on the synchronization properties is discussed in Subsec. 4.2.2.

B A much more complicated situation arises for the SNIPER model for intermediate to small delay times. Here, the stable region might be a deformed cycle but can also consist of several disconnected islands. A rigorous study for all values of delay times and coupling strengths is therefore impossible. However, studying a few example values already gives valuable insight into the possible scenarios. This is done in Subsec. 4.2.3.

\subsubsection{Network models}

The network models that is investigated in the following consist of inhibitory and excitatory links. From a biological point of view this is debatable as it is known that a neuron releases, with rare exceptions, the same set of neurotransmitters at all of its synapses meaning that it either acts excitatorily or inhibitorily [Dale, 1935; Eccles, 1976] but not both at the same time. Thus, a model with excitatory or inhibitory nodes instead of links would be more accurate. However, it has been shown that the results are qualitatively similar if using excitatory or inhibitory links instead of nodes [Schwarze, 2014], though for small delays differences are observable as will be discussed later.

We construct the small-world network of $N$ nodes as follows:

1. We begin with a regular ring network of bidirectional, excitatory links, where each node is connected with equal strength to its $k$ nearest neighbors to the left and to the right. This results in $k N$ excitatory links in the network.

2. For each of those $k N$ excitatory links, we add with probability $p$ a bidirectional, inhibitory link connecting two randomly chosen nodes.

The construction of the random network of $N$ nodes involves the following steps:

1. A random network of $k N$ bidirectional, excitatory links of equal strength is constructed as described in Subsec. 2.3.2.

2. As in the case of the small-world network discussed above, a bidirectional, inhibitory link is added with probability $p$ for every existing excitatory link. Starting and ending node are chosen randomly. 
(a)

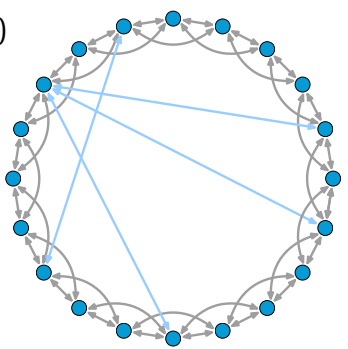

(b)

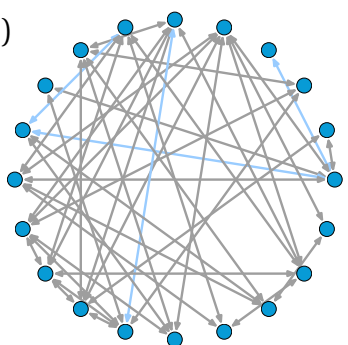

Figure 4.6.: Schematic view of networks consisting of excitatory (gray arrows) and inhibitory links (blue arrows). (a) Small-world type: Regular ring structure of excitatory coupling and additional randomly added long-range inhibitory links. (b) Random excitatory network with additional inhibitory links. Parameters: $N=20, k=2$, $p=0.1$. Number of excitatory links $E=40$, number of inhibitory links $I=4$. Modified from [Lehnert et al., 2011a].

Note that for both models all links are chosen to be bidirectional. A schematic diagram of (a) the small-world network, and (b) the random network is shown in Fig. 4.6. Note that in both model $p$ denotes the average ratio between excitatory and inhibitory links since the number of excitatory links $E$ is given by $k N$, and the expectation value $\langle I\rangle$ of inhibitory links by $p k N$.

This construction of the networks creates a coupling matrix $\mathbf{G}$ (see Sec. 2.1) with the entries 1 (excitatory links) and -1 (inhibitory links). A normalization follows this construction to ensure unity row sum (see Subsec. 3.1). To this end, the $j$ th row, $j=0, \ldots, N-1$, is divided by the absolute value of its row sum $\left|g_{j}={ }_{i} G_{j i}\right|$ unless this sum is zero. Afterwards, we proceed as follows: If $g_{j}<0$, we add a self feedback strength of 2 , i.e., $G_{j j}=2$. If $g_{j}=0$, a self-feedback of strength 1 is introduced, i.e., $G_{j j}=1$. We divide by $\left|g_{j}\right|$ and not by $g_{j}$ because we want to avoid that in the case of a negative row sum all entries of the corresponding row flip their sign and, thus, excitatory links become inhibitory ones and vice versa.

4.2.2 Type-II excitability for arbitrary delays and type-I excitability for large delay times

The evaluation of the stability of type-II (FitzHugh-Nagumo system) and of type-I (SNIPER model) excitable systems for large delay times is straightforward since it is sufficient to check if the eigenvalue spectrum of a given network is contained by the unit circle. Recall that in theses cases the unit circle coincides with the stable region of the MSF (see 


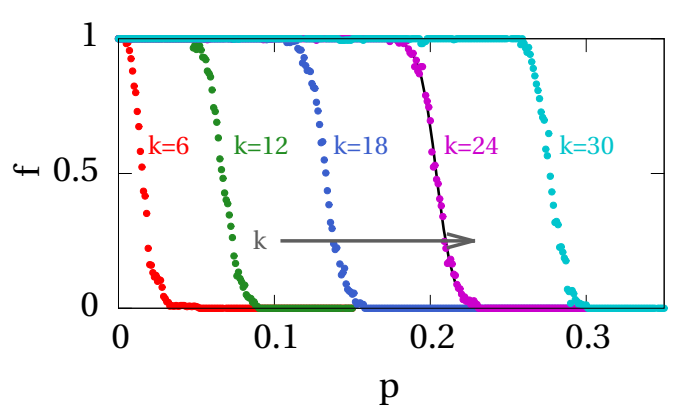

Figure 4.7.: Fraction $f(p)$ of synchronized networks $f$ vs. the probability of additional inhibitory links $p$ for MSFs where the stable region is the unit cycle. $N=100 . k$ varies from 6 to 30 . Thin black curve: Example fit of $f(p)$ according to Eq. (4.12) ( $p_{c}=0.20387$, $\left.k_{s}=186\right)$ for $k=24.500$ realizations for each value of $k$. Figure modified from [Lehnert et al., 2011a].
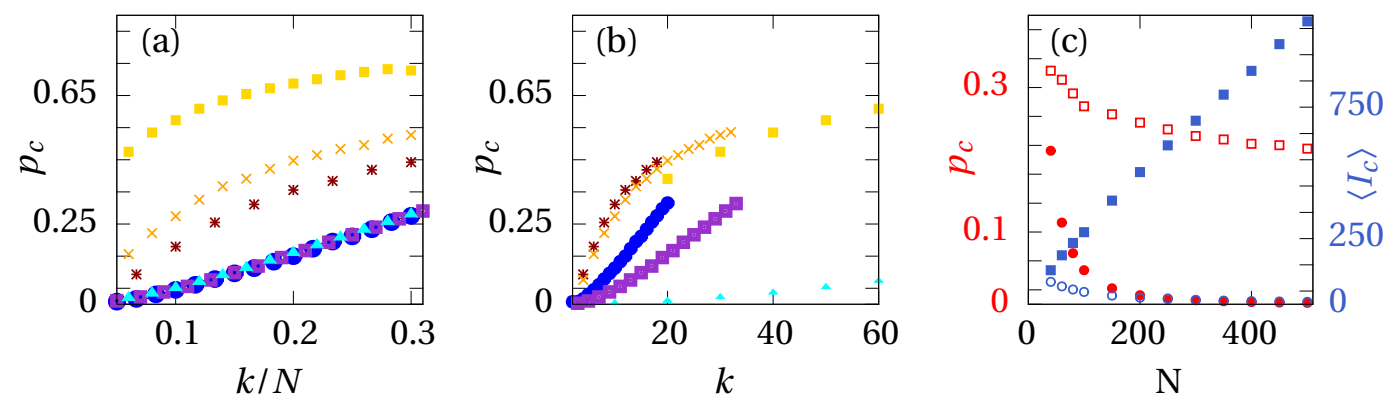

Figure 4.8.: Critical value $p_{c}$ for different network sizes, (a) in dependence on $k / N$, (b) in dependence on $k$ : small-world networks with $N=60$ (blue circles), $N=100$ (purple squares), and $N=500$ (turquoise triangles). Random networks with $N=60$ (red crosses), $N=100$ (orange circles), and $N=500$ (yellow squares). (c) $p_{c} v s . \quad N$ for $k=10$ for a small-world network (red filled circles) and a random network (red empty squares); number of inhibitory links $\left\langle I_{c}\right\rangle$ vs. $N$ for constant $k$ for a small-world (blue empty circles) and a random (blue filled squares) network. 500 realizations for each data point. Figure modified from [Lehnert et al., 2011a]. 
Sec. 4.1). In [Lehnert, 2010; Lehnert et al., 2011a], it was shown that for excitatory coupling matrices, i.e., all entries are positive or zero, with a unity row sum the eigenvalues are always inside the unit circle and, thus, synchronization in these networks will always be stable. In conclusion, inhibition is necessary for a desynchronization process.

Since inhibition is added in a random manner in the networks discussed here (see Subsec. 4.2.1), it is useful to consider the fraction $f$ of synchronized networks for each parameter set instead of considering single realizations. Figure 4.7 depicts $f(p)$ vs. the probability $p$ of additional inhibitory links for different coupling ranges $k$. Obviously, a steep transition between synchronization and desynchronization takes place as $p$ approaches a critical value $p_{c}$. In the following, we define $p_{c}$ as the value where half of the realizations are desynchronized, i.e, $f\left(p_{c}\right)=0.5$.

The critical value $p_{c}$ and the steepness $k_{s}$ of the transition can be fitted with a sigmoidal function

$$
f(p)=1 /\left[e^{-k_{s}\left(p-p_{c}\right)}+1\right] .
$$

Figure 4.8(a) depicts the critical probability $p_{c}$ in dependence on $k / N$ for different network sizes. It can be seen that for small-world networks (blue, purple, and turquoise symbols), $p_{c}$ follows a linear relation $p_{c}(k / N)=1.16 k / N-0.07$ independently of the network size $N$. In contrast, the curves of the random network (yellow, orange, and red symbols) show a slight curvature and no data collapse for this depiction. Figure 4.8(b) is identical to Fig. 4.8(a) but with $k$ instead of $k / N$ on the $x$-axis. Here, it can be seen that the critical probability $p_{c}$ in the random networks is a function of $\log k$.

A distinctly different behavior of small-world and random networks can be observed when considering $p_{c}$ in dependence on the network size $N$ as done in Fig. 4.8(c) for constant $k=10$ : Red squares denote the random network, while red circles stand for the small-world network. For random networks, $p_{c}$ is independent of $N$ for sufficiently large $N$, while for small-world networks it approaches zero. Recall that $p_{c}$ is the mean value of the ratio of inhibitory to excitatory links. In conclusion, in small-world networks with increasing network size $N$ but same local structure (constant $k$ ) an infinitesimally small ratio of inhibition to excitation is sufficient to desynchronize the network, while in a random network even for very large networks only a non-vanishing ratio impairs synchronization.

This effect is also reflected by the number of inhibitory links needed to desynchronize the network. The average number of inhibitory links can be calculated as $\langle I\rangle=p k N$. 
Figure 4.8(c) shows the critical $\left\langle I_{c}\right\rangle=p_{c} N K$ to desynchronize the network for a smallworld (blue circles) and a random (blue squares) network. For the small-world network, $\left\langle I_{c}\right\rangle$ scales as $\langle I\rangle=1.16 k^{2}-0.07 k N$ for small $N$ and approaches zero for large $N$, while for the random network $\langle I\rangle$ is proportional to $N$, i.e., an increasing number of inhibitory links is needed to achieve desynchronization. The behavior can be intuitively understood: In a small-world network each inhibitory link acts as short cut significantly decreasing the shortest path length compared to the underlying regular ring. Thus, perturbations travel often along the inhibitory link where they might be reinforced. In contrast, the shortest path length in a random network is low from the beginning. Thus, the impact of a inhibitory link is much weaker, as the information coming from this link can be balanced by the information coming from other short paths consisting of excitatory links.

\subsubsection{Type-I excitability for small delay times}

In the previous Subsection, I have discussed the impact of inhibitory links on networks for cases where the stable region coincides with the unit circle. As pointed out in Subsec. 4.1.3, the situation is much more complicated for the SNIPER model with small delay time as the stable region of the MSF can take on very different shapes and might even split into several disconnected islands depending on the choice of the coupling parameter. Consequently, a study of the transition behavior can only be done for example values. In [Keane et al., 2012], the case $K=0.3$ and $\tau=6.5$ was investigated because here the stable region has two separated islands (see Fig. 4.3(b)), a form allowing for particular interesting synchronization-desynchronization transitions as eigenvalues may wander in and out of the stable region while increasing the probability $p$ of inhibitory links.

Figure 4.9 shows three different transition scenarios for the small-world network: In panel (a), (b), and (c) the fraction $f$ of synchronized networks is shown for different coupling ranges of (a) $k=20$, (b) $k=40$, and (c) $k=50$ is shown. Panels (d), (e), and (f) show the stable region and eigenvalue spectrum for the scenarios in panels (a), (b), and (c), respectively. Clearly, much more complicated transitions than the ones discussed in the previous Subsection are possible including scenarios where multiple transitions between synchronization and desynchronization take place (see panels (b) and (c)).

For random networks, the situation is different. Here, at most one transition can be found, similar to the transitions depicted in Fig. 4.7. Furthermore, parameter values of $k$ 

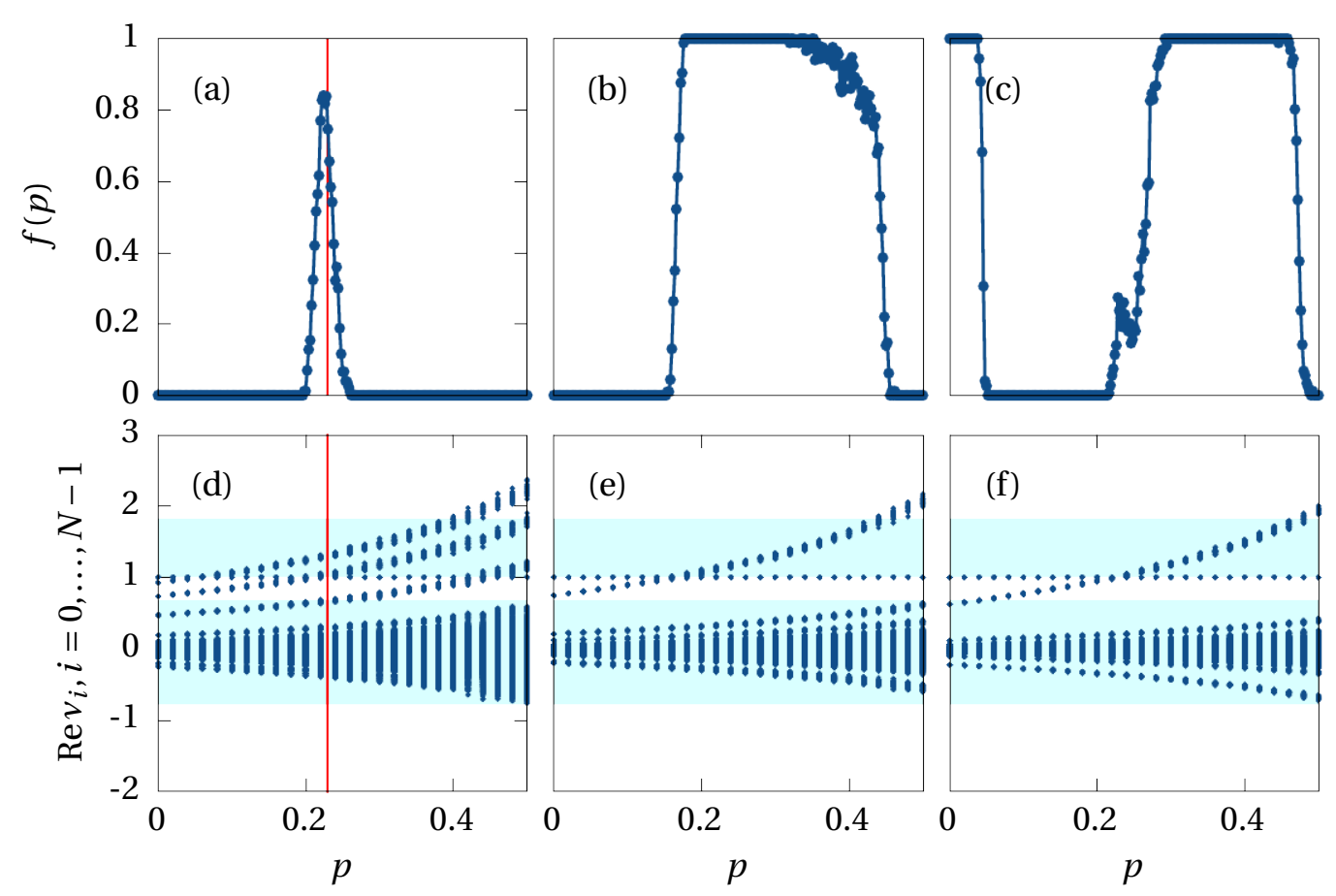

Figure 4.9.: Fraction of synchronized networks $f$ in dependence on the probability of additional inhibitory links $p$ for coupling ranges of (a) $k=20$, (b) $k=40$, and (c) $k=50$ with 500 realization for each $p$ value. Corresponding eigenvalue spectra for $(d)$ $k=20$, (e) $k=40$, and (f) $k=50$ with 50 realizations for each $p$ value. The turquoise shaded regions represent the stable regions of the real part of the MSF. Red line in panels (a) and (d) marks the parameters used in Fig. 4.10. Parameters: $N=200, K=0.3$, $\tau=6.5, b=0.95$. Figure reproduced from [Keane et al., 2012]. 

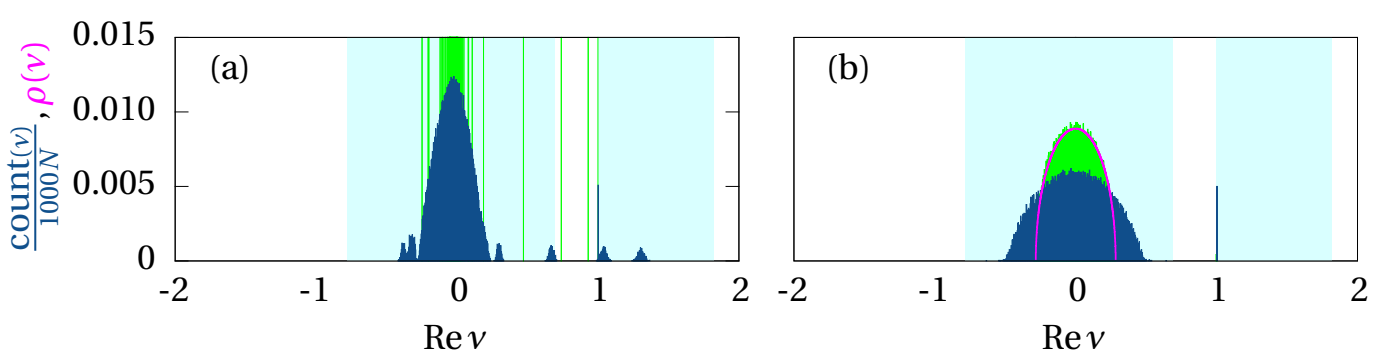

Figure 4.10.: Normalized histograms (depicted in blue) of eigenvalue spectra for 1000 realizations of (a) a small-world network and (b) a random network with the same number of nodes, $N=200$; the same number of excitatory links, $k=20$; and the same probability of inhibitory links, $p=0.23$. Turquoise shading: Stable region of the MSF for $K=0.3$ and $\tau=6.5$. Green lines in (a): Position of eigenvalues of a small-world network for $p=0$ according to Eq. (2.15). Green histogram in (b): histogram of eigenvalue spectra for 1000 realizations of a random network for $p=0$. Pink line in (b): eigenvalue distribution $\rho(v)$ according to Eq. (4.14). Number of bins is 1000. Figure reproduced and extended from [Keane et al., 2012].

and $N$ exist where the network is already desynchronized for $p=0$ and no transition to synchronization takes place with growing $p$.

This difference between small-world and random networks can be explained by considering the histograms of the eigenvalue spectra of (a) the small-world and (b) the random network as done in Fig. 4.10. The histograms are normalized such that they coincide in the limit of large $N$ with the eigenvalue distribution. Clearly, the eigenvalue spectrum of the small-world network is characterized by several peaks. Appropriate choice of parameters allows for shifting these peaks into the stable region (turquoise shaded). The network parameter, $k, N, p$, are such that they correspond to the peak of $f$ seen in Fig. 4.9(a) (red line there). Thus, almost all eigenvalues lay inside the stable region. In contrast, the eigenvalues of the random network form a bulk, from which only the longitudinal eigenvalue $v=1$ is separated. Increasing $p$ only enlarges the width of the bulk so that at the critical value $p_{c}$, the eigenvalues enter the unstable region and desynchronization takes places. However, no resynchronization is possible since no peaks exist which could be shifted until they fit the stable regions.

The qualitatively very different eigenspectra of random and small-world network can be understood if considering the networks as perturbation from the networks without inhibition, i.e., $p=0$. In the case of the small-world network the network without in- 
hibition is a regular ring network with $k$ nearest neighbors which eigenvalues are given by

$$
v_{j}=\frac{1}{k_{l=1}^{k}} \cos \frac{2 \pi j l}{N}
$$

(see also Eq. (2.15)). These eigenvalues are depicted as green, vertical lines in Fig. 4.10.

As discussed in Sec. 2.3.2 the eigenvalues of an undirected random network are given by Wigner's law or semicircle law [Wigner, 1958]. However, here we have to take the normalization of the coupling matrix into account. Thus, we have to multiply the eigenvalue $v$ in the original formula, see Eq. (2.19), by the average row sum $q(N-1)$ to get the correct form of the new distribution. Recall that $q$ is the probability that an excitatory link exists between two randomly chosen links. Thus in the limit of large $N, q=2 k /(N-1)$ holds, since the excitatory part of the random network consists of $2 k$ links. Furthermore, we have to divide $\rho(v)$ by the factor $\overline{q(N-1)}$ to keep the correct normalization of $\rho(v)$. Then the eigenspectrum is described by

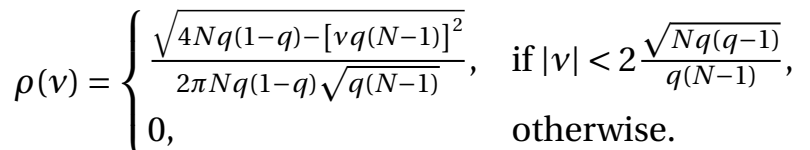

In Fig. 4.10(b) the eigenvalue spectrum of random network without inhibition is depicted as a green histogram; the pink line marks semicircle law $\rho(v)$ as given by Eq. (4.14). Obviously, the eigenspectra of the small-world and the random networks for $p>0$ qualitatively resemble the ones for $p=0$ but are blurred around their original position and stretched along the $x$-axis. For a very detailed and comprehensive study of the eigenvalue spectra of small-world networks and random networks on the network parameters see [Schwarze, 2014]. In particular, Schwarze studies the influence of inhibition on the eigenspectra.

Note that the results presented in Figs. 4.9 and 4.10 slightly differ from the ones presented in [Keane et al., 2012]. This is because Keane et al. used a different normalization procedure: They divided each row by the row sum $g_{j}$ instead of its absolute value $\left|g_{j}\right|$. Realizations with zero row sum were discarded. For reasons of consistency, I use here the normalization explained in Subsec. 4.2.1 which was also used in [Lehnert et al., 2011a]. Qualitatively the results of both normalization methods agree. 


\subsection{CONCLUSION}

In this Chapter, the stability of synchronization in excitable system has been studied. According to the underlying bifurcation, two types of excitability can be distinguished: Type-I excitability is observed close to a saddle-node infinite period (SNIPER) bifurcation, while type-II excitability is induced by a supercritical Hopf bifurcation. Here two generic models have been considered: The normal form of the SNIPER bifurcation for type-I excitability and the FitzHugh-Nagumo system for type-II excitability. These models have been used as local dynamics in a delayed coupled network, where the coupling parameter have been chosen such that they induce oscillations in the synchronous manifold though the uncoupled systems are excitable.

The stability of synchronization can be evaluated with the help of the master stability function (MSF). Qualitatively, we have found two different classes of the MSF: (A) MSFs where the stable region is given by the unit circle, and (B) MSFs where the stable region has a much more complicated form and might even split into several disconnected islands.

MSFs of class (A) have been found for the SNIPER model for large delays which is congruent with the result of Flunkert et al. [2010] who proofed that in the limit of infinite delay times the stable region of the MSF coincides with the unit circle if the synchronous dynamics is oscillatory. Surprisingly, the MSF for the FitzHugh-Nagumo is of the same class, fairly independently of the coupling parameters $K$ and $\tau$ as long as they a large enough to induce oscillations. The most probable explanation for this behavior is that the delay might always be considered to be "large" because the delay time sets the oscillation period of the coupled FitzHugh-Nagumo system [Schöll et al., 2009; Dahlem et al., 2009]. Consequently, delay and period are always of the same order of magnitude and the results of Flunkert et al. might already be applicable. For small delays, the SNIPER model shows the more complicated MSFs of class (B).

Based on the MSF, synchronization in small-world and random networks with inhibition has been investigated. For MSFs of class (A), i.e., the stable region is a unit circle, one transition from synchronization to desynchronization has been found when the probability of additional inhibitory links reaches a critical value. The behavior of small-world and random networks is distinctly different in the limit of large $N$. In particular, an infinitesimally small ratio of inhibition is sufficient to destabilize synchronization in a large small-world network, while in a random network always a non-vanishing ratio is needed to induce desynchronization. The MSF of class (B) allows for multiple transitions from synchronization to desynchronization and vice versa in the small-world network depending on the coupling parameters. In random networks, at most one transition can 
take place for the same MSF. These differences are explained by the different eigenspectra of the networks.

The here presented network models consist of excitatory and inhibitory links. Biologically more plausibility could be reached by using excitatory and inhibitory nodes rather than excitatory and inhibitory links, i.e., by constructing the network such that the links outgoing from the same node are always of the same kind. This was studied in [Schwarze, 2014]. Schwarze found that the transitions for MSFs of class (A) are qualitatively very similar to the network model presented here. However, in the case that the MSF is of class (B), Schwarze did not observed multiple transitions but transitions that are similar to the ones observed for class (A) or transitions from desynchronization to a regime where roughly $20 \%$ of the realizations synchronize.

An interesting question is how generic the results obtained in this Chapter are for the two types of excitability, in particular, as the dynamics of the FitzHugh-Nagumo system in the excitable regime are not only influenced by the nearby Hopf bifurcation but in addition a canard explosion is observed [Benoit et al., 1981]. Sonnenschein [2013] investigated synchronization-desynchronization transitions in the Morris-Lecar model which shows type-I or type-II excitability depending on the parameter set. For $\tau$ in the order of the system's oscillation, he observed MSFs of class (A) as anticipated from the results of Flunkert et al. [2010] and the discussion in this Chapter. However, as $\tau$ decreases qualitatively very different MSFs can be observed for the type-I and the type-II regime. These MSFs include situations similar to two separated islands as shown in Fig. 4.2(b) but also cases where no stable region exists along the $x$ axis, and, thus, most network not constructed with the explicit goal to fit these stable regions will desynchronize.

In conclusion, the synchronization-desynchronization transitions observed for the MSFs of class (A) seem to be generic for models where the delay time is of the same order of magnitude as the system's oscillation. Furthermore, they seem to be robust towards changes in the construction procedure of the networks. However, as soon as the delay becomes small no general conclusion is possible but the considered model, the coupling parameters, and the details of the network construction have to be taken into consideration. Therefore, the results obtained in [Keane et al., 2012] and discussed in Sec. 4.2.3 can only be seen as exemplary findings showing the variety of different transitions which might exist.

This variety of possible synchronization-desynchronization transitions is further increased when considering distributed delays: Wille et al. [2014] investigated the combined effects of distributed delay and the balance between excitatory and inhibitory nodes on networks of coupled Stuart-Landau oscillators (for a description of the Stuart-Landau oscillator see Subsec. 5.2). They found that both the delay distribution width as well 
4. CONTROL OF SYNCHRONIZATION TRANSITIONS BY BALANCING EXCITATORY AND INHIBITORY COUPLING

as the inhibition ratio can induce synchronization-desynchronization transitions. For small delay distribution widths, strong inhibition causes desynchronization. Furthermore, Wille et al. showed that distributed delay tends to enhance the possibility of stable synchronization for increasing inhibition ratios. 


\section{5 | CLUSTER AND GROUP SYNCHRONY: THE THEORY}

In Chapters 3 and 4, I discussed the stability of zero-lag synchronization which was the main focus of research concerning the dynamics on networks for a long time. Recently, more complex synchronization patterns, including cluster and group synchronization, have received growing interest both in theory [Sorrentino and Ott, 2007; Kestler et al., 2007; Ashwin et al., 2007; Kestler et al., 2008; Kori and Kuramoto, 2001; Lücken and Yanchuk, 2012; Dahms et al., 2012; Kanter et al., 2011b,a; Golubitsky and Stewart, 2002; Sorrentino, 2014; Pecora et al., 2014; Poel et al., 2015] and in experiments [Illing et al., 2011; Aviad et al., 2012; Blaha et al., 2013; Rosin et al., 2013; Williams et al., 2012, 2013a,b; Rosin, 2015]. These scenarios appear in many biological systems, examples include dynamics of nephrons [Mosekilde et al., 2002], central pattern generation in animal locomotion [Ijspeert, 2008], or population dynamics [Blasius et al., 1999].

We define group and cluster synchronization as follows [Dahms et al., 2012]:

Cluster synchronization: In an $M$-cluster state, the compound system evolves in $M$ clusters with zero-lag synchrony between the nodes of one cluster and - in the case of an oscillatory system - with a constant phase lag between the clusters. The local dynamics of each cluster is given by the same sets of equations.

Group synchronization: Group synchronization refers to the case where each group potentially exhibits different local dynamics. Thus, group synchronization can be considered as a generalization of cluster synchronization.

For simplicity, I use in the following the term group synchrony to refer to group and cluster synchrony if it is not necessary to distinguish between the two.

The aim of this Chapter is to discuss group synchronization in complex networks. For zero-lag synchronization, the MSF has proven to be a valuable tool to evaluate the stabil- 
ity of the synchronization manifold (cf. Chaps. 3 and 4). Section 5.1 extends the MSF for group synchronization: Firstly, it is discussed which network topologies allow for group synchrony before the generalized MSF is derived. The eigenvalue spectra of networks capable to exhibit group synchrony as well as the MSF for this type of dynamics show a discrete rotational symmetry which is the subject of Subsecs. 5.1.3 and 5.1.4. Section 5.2 is a recapitulation of the analytic results on cluster synchrony in coupled Stuart-Landau oscillators obtained in [Choe et al., 2010]. A further extension of the MSF for non-smooth systems including group synchronization in such systems is given in Sec. 5.3. There, the applicability of the MSF is demonstrated on the example of adaptive exponential integrate-and-fire neurons.

\subsection{MASTER STABILITY FUNCTION FOR GROUP AND CLUSTER SYNCHRO- NIZATION}

Sorrentino and Ott [2007] suggested an MSF for group synchronization of two groups. A generalization to an arbitrary number of groups was developed in [Lehnert, 2010; Dahms, 2011; Dahms et al., 2012] and is discussed in this Section.

\subsubsection{Restrictions on the topology}

In contrast to zero-lag synchronization which is a solution of a dynamical system of the form (3.4) for all coupling matrices $\mathbf{G}$ if the constant row sum conditions holds (see Subsec. 3.1), group synchronization imposes certain requirements on the network topology. Several approaches have been developed to determine whether a network topology allows for a group synchrony solution. Kanter et al. [2011b,a] suggested the Greatestcommon-divisor (GCD) criteria. However, this criteria is only applicable to networks consisting of one or several loops. A very comprehensive answer to the question which networks might exhibit group synchrony is given by Pecora et al. [2014]. Their method is based on groups theory and investigates the symmetries of the network. The advantage is that it can be applied to any network topology to calculate the number of groups which can range from 1 (zero-lag synchrony) to $N$ (a splay state). The disadvantage is that it involves advanced group theoretical methods and is therefore not easy to handle. Furthermore, it requires the coupling matrix to be unweighted, i.e, to consist of 1's and 0's only, where a generalization to weighted matrices might be possible.

Here, we require that each of the $M$ groups couples to only one other group. This means that the network is a unidirectional ring seen from the level of groups. In this Chapter, we 
refer to a network fulfilling this criteria as an $M$-partite graph. Note that this definition of an $M$-partite graph is more strict than the more commonly used definition which only requires that the nodes of the same group do not couple [Kivelä et al., 2014]. In the case of a bipartite network, both definitions coincide. A coupling matrix $\mathbf{G}$ allowing for synchronization in an $M$-group state has then the following block form

$$
\mathbf{G}=\begin{array}{ccccc}
0 & \cdots & \cdots & 0 & \mathbf{A}^{(0)} \\
\mathbf{A}^{(1)} & 0 & \cdots & \cdots & 0 \\
0 & \mathbf{A}^{(2)} & 0 & \cdots & 0 \\
0 & \ddots & \ddots & \ddots & 0 \\
0 & \cdots & 0 & \mathbf{A}^{(M-1)} & 0
\end{array},
$$

where the $N_{k} \times N_{k-1}$ matrix $\mathbf{A}^{(k)}$ describes how group $k-1$ couples to group $k$. Here and in the following, all indices referring to the groups are taken modulus $M . N_{k}$ is the number of nodes in group $k$; note that we do not require equally sized groups. In Eq. (5.1) it is assumed that the nodes are ordered according to their group: The first $N_{0}$ nodes belong to cluster 0 , the following $N_{1}$ nodes to cluster 1 and so on. For notational convenience, it is in the following assumed that $N_{0} \leq N_{j}(j=2, \ldots, M)$, which can always be achieved by an index permutation. Note that - after a renumbering of the nodes a coupling matrix of form (5.1) includes the loop networks suggested by Kanter et al. [2011b,a].

\subsubsection{Derivation of the MSF for group synchrony}

In [Dahms, 2011; Dahms et al., 2012], we derived an MSF to evaluate the stability of synchronization in $M$ groups was derived which is discussed in this Subsection.

If the overall coupling matrix is of the form (5.1), the dynamics of the $i$ th node in the $k$ th group are described by

$$
\dot{\mathbf{x}}_{i}^{(k)}=\mathbf{f}^{(k)}\left(\mathbf{x}_{i}^{(k)}\right)+\sigma_{j=0}^{(k)} A_{i j}^{N_{k-1}-1} \mathbf{H}^{(k)} \mathbf{x}_{j}^{(k-1)}\left(t-\tau^{(k)}\right)-\mathbf{x}_{i}^{(k)}(t),
$$

for $i=0, \ldots, N_{k}-1, k=0, \ldots, M-1 . \mathbf{f}^{(k)}$ is the local dynamics for all nodes in group $k$ and of dimension $d_{k}$. Note that group synchrony does not require that all groups follow the same local dynamics. For example, groups of inhibitory and excitatory neurons are considered in Sec. 5.3 where each group is governed by different local dynamics. In contrast, 
cluster synchrony takes place only in networks where that the local dynamics are identical for all groups, i.e, $\mathbf{f}^{(0)}, \ldots, \mathbf{f}^{(M-1)} \equiv \mathbf{f} . \mathbf{H}^{(k)}$ is a $d_{k} \times d_{k-1}$ matrix describing the coupling scheme from group $k-1$ to group $k . \sigma^{(k)}$ describes the overall strength of the coupling from group $k-1$ to group $k$. Analogously, $\tau^{(k)}$ is the time delay between group $k-1$ and group $k$. Thus, the coupling described by Eq. (5.2) allows for different coupling strengths and delay times between the groups.

Group synchrony requires that the input to each node is equal to one in the group synchronized case. This is equivalent to a unity-row sum condition for each matrix $\mathbf{A}^{(k)}$, i.e., $\quad{ }_{j} A_{i j}^{(k)}=1$ for $i=0, \ldots, N-1$ and $k=0, \ldots, M-1$. Constant row sums not equal to one can be rescaled with the respective coupling strengths: Assume that the ma$\operatorname{trix} \mathbf{A}^{(k)}$ has a row sum equal to $a^{(k)} \neq 1$. If we rescale both, $\mathbf{A}^{(k)}$ and $\sigma^{k}$, as $\mathbf{A}^{(k)} \rightarrow$ $\mathbf{A}^{(k)} / a^{(k)}$ and $\sigma^{(k)} \rightarrow a^{(k)} \sigma^{(k)}$, we obtain a unity row without changing the effective overall coupling strength. Thus, we can assume without loss of generality that the row sum is one.

Note that in [Dahms et al., 2012] a non-diffusive coupling of the form ${ }_{j} A_{i j}^{(k)} \mathbf{H}^{(k)} \mathbf{x}_{j}^{(k-1)}(t-$ $\left.\tau^{(k)}\right)$ was used which is, for example, applicable to lasers [Lang and Kobayashi, 1980] or optoelectronic oscillators [Illing et al., 2011]. Since the focus of this thesis are neural systems, we here use diffusive-like coupling term of the form

$$
{ }_{j} A_{i j}^{(k)} \mathbf{H}^{(k)}\left[\mathbf{x}_{j}^{(k-1)}\left(t-\tau^{(k)}\right)-\mathbf{x}_{i}^{(k)}(t)\right]
$$

Introducing

$$
\mathbf{F}^{(k)}=\mathbf{f}^{(k)}\left(\mathbf{x}_{i}^{(k)}\right)-\sigma^{(k)} \mathbf{H}^{(k)} \mathbf{x}_{i}^{(k)},
$$

system (5.2) is transformed to

$$
\dot{\mathbf{x}}_{i}^{(k)}=\mathbf{F}^{(k)}\left(\mathbf{x}_{i}^{(k)}\right)+\sigma_{j=0}^{(k)} A_{i j}^{N_{k-1}-1} \mathbf{H}^{(k)} \mathbf{x}_{j}^{(k-1)}\left(t-\tau^{(k)}\right) .
$$

which is equivalent to the system considered in [Dahms et al., 2012]. Thus, from a mathematical point of view no difference between the two types of coupling exist.

Substituting $\mathbf{x}_{i}^{(k)}=\mathbf{x}_{j}^{(k)} \equiv \mathbf{x}_{s}^{(k)}$ into Eq. (5.2), for $i, j=0, \ldots, N_{k}-1$ and $k=0, \ldots, M-1$, yields the differential equation describing the dynamics in the group synchronized state

$$
\dot{\mathbf{x}}_{s}^{(k)}=\mathbf{F}^{(k)}\left(\mathbf{x}_{s}^{(k)}\right)+\sigma^{(k)} \mathbf{H}^{(k)} \mathbf{x}_{s}^{(k-1)}\left(t-\tau^{(k)}\right),
$$


(a)

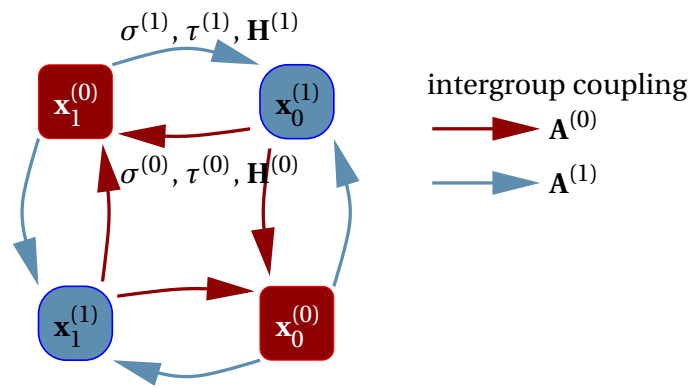

(b)

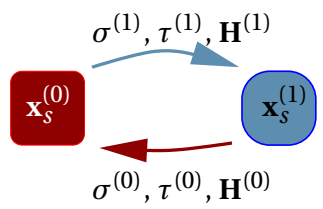

Figure 5.1.: (a) Schematic diagram of two groups visualizing parameters and dynamical variables as in Eq. (5.2) for multipartite topologies. (b) The corresponding synchronization manifold according to Eq. (5.6). Figure modified from [Dahms et al., 2012].

which corresponds to a network of $k$ nodes coupled in a unidirectional ring. Note that even if $\mathbf{F}^{(k)}, \mathbf{H}^{(k)}, \sigma^{(k)}$, and $\tau^{(k)}$ are identical for all groups, i.e., independent of $k$, the different groups do not necessarily follow the same dynamics but different initial conditions may yield different dynamics in the group synchrony state.

Figure 5.1(a) is a schematic diagram of two groups each consisting of two nodes couppled according to Eq. (5.2). The multipartite structure of the network is clearly visible since no coupling between the nodes of one group exists. Panel (b) shows the corresping synchronization manifold. In the synchronization manifold, the multipartite coupling translates to a coupling in a unidirectional ring. In the case of two groups, the synchronization manifold is a motif of two nodes coupled to each other but without selffeedback.

Linearizing system (5.5) at the synchronized dynamics given by Eq. (5.6) yields the variational equation

$$
\delta \dot{\mathbf{x}}_{i}^{(k)}=D \mathbf{F}^{(k)}\left(\mathbf{x}_{s}^{(k)}\right) \delta \mathbf{x}_{i}^{(k)}+\sigma_{j=0}^{(k)} A_{i j}^{N_{k-1}-1} \mathbf{H}^{(k)} \delta \mathbf{x}_{j}^{(k-1)}\left(t-\tau^{(k)}\right)
$$

or in block form

$$
\delta \dot{\mathbf{x}}^{(k)}=\mathbb{1}_{N_{k}} \otimes D \mathbf{F}^{(k)}\left(\mathbf{x}_{s}^{(k)}\right) \delta \mathbf{x}^{(k)}+\sigma^{(k)} \mathbf{A}^{(k)} \otimes \mathbf{H}^{(k)} \delta \mathbf{x}^{(k-1)}\left(t-\tau^{(k)}\right) .
$$

Here, $\mathbb{1}_{N_{k}}$ is a unity matrix of dimension $N_{k}$.

In the following, we conduct a basis transformation such that Eq. (5.8) decouples to a 
form which can be considered as an MSF. The derivation of the MSF discussed here differs from the one presented in [Sorrentino and Ott, 2007; Dahms, 2011; Dahms et al., 2012]. The aim is to find a derivation which is easier accessible by using standard linear algebra techniques.

We introduce the following basis transformation for each group:

$$
\delta \overline{\mathbf{x}}^{(k)}=\left(\mathbf{C}^{(k)}\right)^{-1} \otimes \mathbb{1}_{d_{k}} \delta \mathbf{x}^{(k)},
$$

where $\mathbf{C}^{(k)}$ is a time independent $N_{k} \times N_{k}$ matrix. $\mathbb{1}_{d_{k}}$ is a unity matrix of dimension $d_{k}$ with $d_{k}$ being the dimension of the nodes in group $k$.

We choose $\mathbf{C}^{(k)}$ such that

$$
\mathbf{A}^{(k)} \mathbf{C}^{(k-1)}=\mathbf{C}^{(k)} \mathbf{D}^{(k)},
$$

where $\mathbf{D}^{(k)}$ is an $N_{k} \times N_{k-1}$ matrix with $\mathbf{D}_{i j}^{(k)}=v_{i} \delta_{i j}, v_{i} \in \mathbb{C}, i, j=0, \ldots, N_{0}-1$, and zeros otherwise. We will see that $v_{i}$ can be identified with the non-zero eigenvalues of G.

Multiplying Eq. (5.8) from the left with $\left(\mathbf{C}^{(k)}\right)^{-1} \otimes \mathbb{1}_{d_{k}}$ yields

$$
\begin{aligned}
\delta \dot{\mathbf{x}}^{(k)}= & \mathbb{1}_{N_{k} \otimes} D \mathbf{F}^{(k)}\left(\mathbf{x}_{s}^{(k)}\right) \delta \overline{\mathbf{x}}^{(k)} \\
& +\sigma^{(k)}\left(\mathbf{C}^{(k)}\right)^{-1} \otimes \mathbb{1}_{d_{k}} \quad \mathbf{A}^{(k)} \mathbf{C}^{(k-1)}\left(\mathbf{C}^{(k-1)}\right)^{-1} \otimes \mathbf{H}^{(k)} \delta \mathbf{x}^{(k-1)}\left(t-\tau^{(k)}\right) .
\end{aligned}
$$

where we introduced a "one": $\mathbf{C}^{(k-1)}\left(\mathbf{C}^{(k-1)}\right)^{-1}=\mathbb{1}_{N_{k-1}}$. The first term on the right-hand side of Eq. (5.11) was rearranged according to the rules for multiplication with the Kronecker product (see Eq. (B.1) in Appendix B).

Using Eq. (5.10) yields

$$
\begin{aligned}
\delta \dot{\mathbf{x}}^{(k)}= & \mathbb{1}_{N_{k} \otimes} D \mathbf{F}^{(k)}\left(\mathbf{x}_{s}^{(k)}\right) \delta \overline{\mathbf{x}}^{(k)} \\
& +\sigma^{(k)}\left(\mathbf{C}^{(k)}\right)^{-1} \otimes \mathbb{1}_{d_{k}} \quad \mathbf{C}^{(k)} \mathbf{D}^{(k)}\left(\mathbf{C}^{(k-1)}\right)^{-1} \otimes \mathbf{H}^{(k)} \delta \mathbf{x}^{(k-1)}\left(t-\tau^{(k)}\right) .
\end{aligned}
$$

Again applying rule (B.1), it follows

$$
\begin{aligned}
\delta \dot{\mathbf{x}}^{(k)}= & \mathbb{1}_{N_{k} \otimes D \mathbf{F}^{(k)}\left(\mathbf{x}_{s}^{(k)}\right) \delta \overline{\mathbf{x}}^{(k)}} \\
& +\sigma^{(k)} \mathbf{D}^{(k)}\left(\mathbf{C}^{(k-1)}\right)^{-1} \otimes \mathbf{H}^{(k)} \mathbb{1}_{d_{k-1}} \delta \mathbf{x}^{(k-1)}\left(t-\tau^{(k)}\right),
\end{aligned}
$$


making use of $\mathbf{C}^{(k)}\left(\mathbf{C}^{(k)}\right)^{-1}=\mathbb{1}_{N_{k}}$ and $\mathbb{1}_{d_{k}} \mathbf{H}^{(k)}=\mathbf{H}^{(k)} \mathbb{1}_{d_{k-1}}$.

Finally, we derive

$$
\delta \dot{\mathbf{x}}^{(k)}=\mathbb{1}_{N_{k}} \otimes D \mathbf{F}^{(k)}\left(\mathbf{x}_{s}^{(k)}\right) \delta \overline{\mathbf{x}}^{(k)}+\sigma^{(k)} \mathbf{D}^{(k)} \otimes \mathbf{H}^{(k)} \delta \overline{\mathbf{x}}^{(k-1)}\left(t-\tau^{(k)}\right),
$$

where we used Eq. (B.1) another time.

Thus, we have $N_{0}$ blocks - each consisting of $M$ coupled equations - of the form

$$
\delta \dot{\tilde{\mathbf{x}}}_{i}^{(k)}=D \mathbf{F}^{(k)}\left(\mathbf{x}_{s}^{(k)}\right) \delta \tilde{\mathbf{x}}^{(k)}+v_{i} \sigma^{(k)} \mathbf{H}^{(k)} \delta \tilde{\mathbf{x}}_{i}^{(k-1)}\left(t-\tau^{(k)}\right), \quad k=0, \ldots, M-1 .
$$

Furthermore, we obtain $n_{0}={ }_{k}\left|N_{k}-N_{k-1}\right|$ equations of the form

$$
\delta \dot{\tilde{\mathbf{x}}}_{i}^{(k)}=D \mathbf{F}^{(k)}\left(\mathbf{x}_{s}^{(k)}\right) \delta \tilde{\mathbf{x}}^{(k)} .
$$

In conclusion, Eqs. (5.15) and (5.16) qualify as a master stability equation (MSE) for group synchrony. Here, the $v_{i}$ are chosen from the set of non-zero eigenvalues of the block matrix

$$
\mathbf{G}=\begin{array}{ccccc}
0 & \cdots & \cdots & 0 & \mathbf{A}^{(0)} \\
\mathbf{A}^{(1)} & 0 & \cdots & \cdots & 0 \\
0 & \mathbf{A}^{(2)} & 0 & \cdots & 0 \\
0 & \ddots & \ddots & \ddots & 0 \\
0 & \cdots & 0 & \mathbf{A}^{(M-1)} & 0
\end{array}
$$

because Eq. (5.10) is equivalent to the eigenvalue problem $\mathbf{G v}_{i}=v_{i} \mathbf{v}_{i}, i=0, \ldots, N_{0}-1$, where the eigenvector $\mathbf{v}_{\mathbf{i}}$ is given by

$$
\mathbf{v}_{i}=\begin{gathered}
\mathbf{c}_{i}^{(0)} \\
\vdots \\
\mathbf{c}_{i}^{(M-1)}
\end{gathered},
$$

and $\mathbf{c}_{i}^{(k)}$ is the $i$ th column of the matrix $\mathbf{C}^{(k)}$. This can be seen by multiplying $\mathbf{G}$ with the 
$N \times N_{\max }$ matrix $\mathbf{C}$ given by

$$
\mathbf{C}=\begin{array}{cc}
\mathbf{C}^{(0)} & 0_{N_{0}, N_{\max }-N_{0}} \\
\mathbf{C}^{(1)} & 0_{N_{1}, N_{\max }-N_{1}} \\
\vdots & \vdots \\
\mathbf{C}^{\left(k_{\max }\right)} & \vdots \\
\vdots & \vdots \\
\mathbf{C}^{(M-1)} & 0_{N_{M-1}, N_{\max }-N_{M-1}}
\end{array}
$$

where $N$ is the total number of all nodes in the network, i.e., $N={ }_{k} N_{k}$, and $N_{\max }$ the number of nodes in the largest group, which is here labelled as $k_{\max }$, i.e., $N_{\max }=$ $\max _{k} N_{k} .0_{N_{k}, N_{\max }-N_{k}}$ is a $N_{k} \times\left(N_{\max }-N_{k}\right)$ matrix of zeros which makes up for the matrices $\mathbf{C}^{(k)}$ having different numbers of columns. The multiplication yields

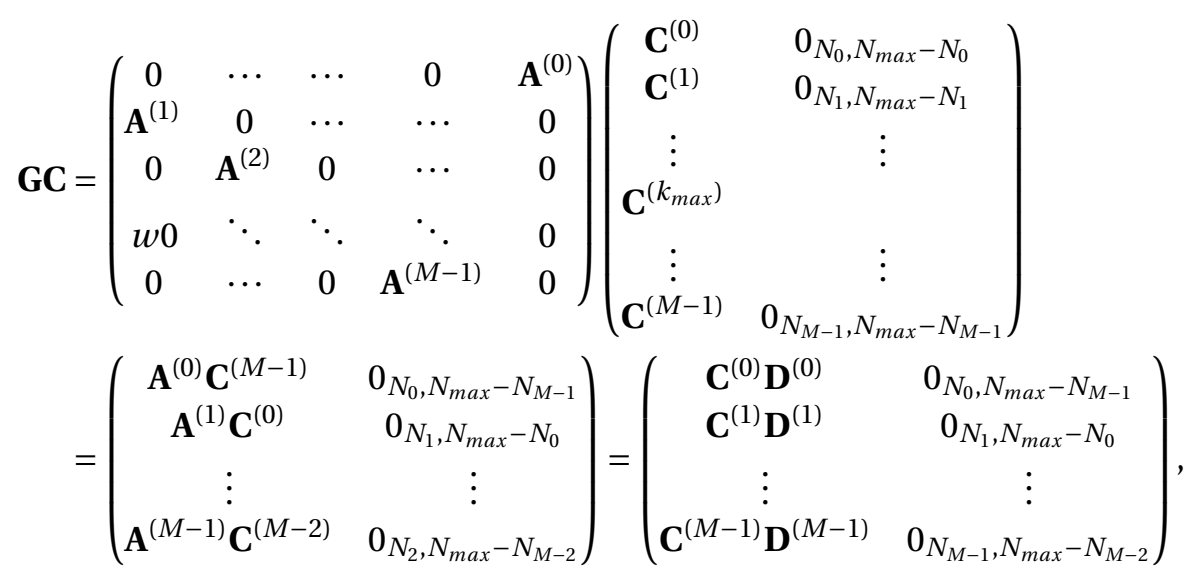

where we used Eq. (5.10) in the last step. Finally, performing the multiplication $\mathbf{C}^{(k)} \mathbf{D}^{(k)}$, we obtain

$$
\mathbf{G C}=v_{0} \mathbf{v}_{0} \quad \ldots \quad v_{N_{0}} \mathbf{v}_{N_{0}} \quad 0_{N, N_{\max }-N_{0}},
$$

meaning that $v_{0}, \ldots, v_{N_{0}}$ are the non-trivial eigenvalues of $\mathbf{G}$.

The largest Lyapunov exponent $\Lambda$ calculated from Eq. (5.15) as a function of the parameter $v \in \mathbb{C}$ is called the MSF. It determines the stability of group synchronization if evaluated at the eigenvalues of the coupling matrix $\mathbf{G}$.

Looking at Eq. (5.15) it seems that we recovered only $N_{0}$ of the possible ${ }_{k} N_{k}$ eigenvalues. However, in Subsecs. 5.1.3 and 5.1.4, it will be shown that - due to a discrete 


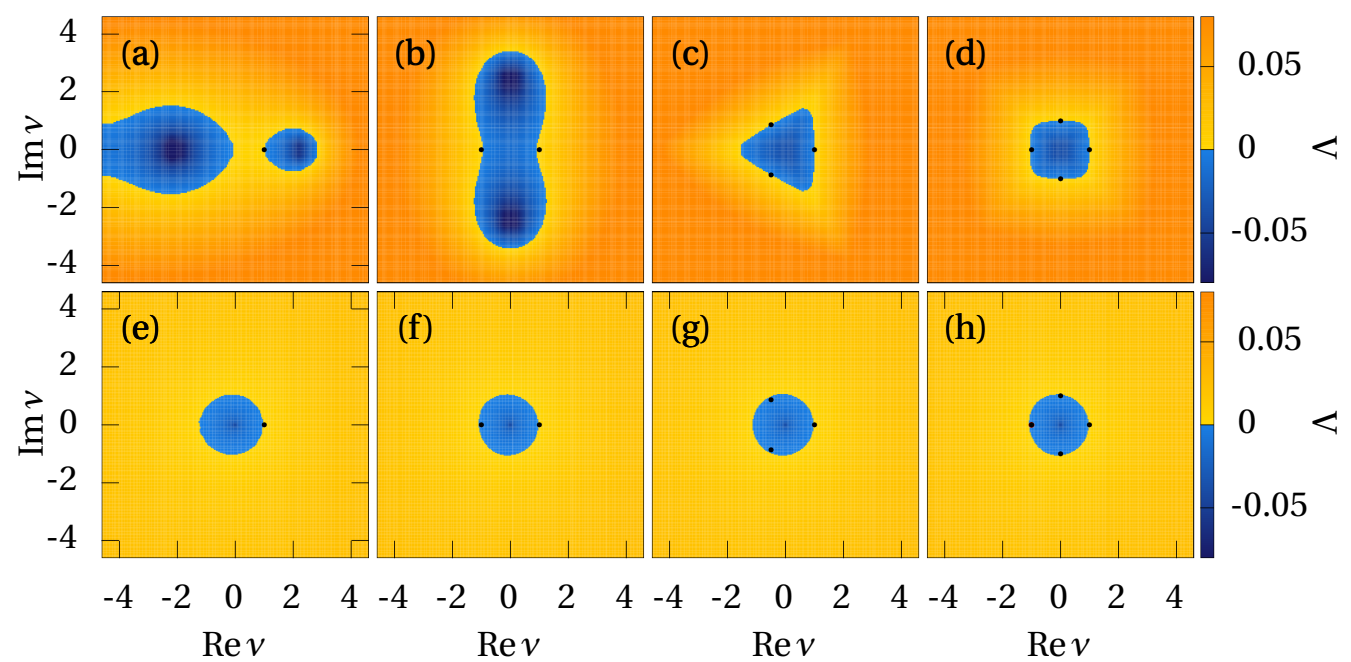

Figure 5.2.: Master stability function (MSF) for group synchrony according to Eq. (5.15) for the SNIPER model (see Sec. 4.1.2, Eq. (4.4)) in the oscillatory regime for $M=1,2,3,4$ in panels (a) and (e), (b) and (f), (c) and (g), and (d) and ( $h$ ), respectively. Black circles mark the longitudinal eigenvalues. Top: $\tau^{(k)} \equiv \tau=3.4$, bottom: $\tau^{(k)} \equiv \tau=60$. Other parameters: $\sigma^{(k)} \equiv \sigma=0.1, b^{(k)} \equiv b=1.05$, where $b^{(k)}$ is the bifurcation parameter of the SNIPER model for the $k$ th group.

rotational symmetry of the eigenvalue spectrum as well as of the MSF - these $N_{0}$ eigenvalues and the eigenvalue 0 , which has a degeneracy of $n_{0}$, are sufficient to determine the stability of group synchrony in a network. The perturbations in the direction of the eigenvalue 0 are described by Eq. (5.16).

\subsubsection{Symmetry of the master stability function}

In [Lehnert, 2010; Dahms et al., 2012], we have shown that the MSF is invariant with respect to the transformation $v \rightarrow \exp (-2 \pi \mathrm{i} / M) v$. In Eq. (5.15) this tranformation yields

$$
\begin{aligned}
\delta \dot{\mathbf{x}}^{(k)} & =D \mathbf{F}^{(k)}\left(\mathbf{x}_{s}^{(k)}\right) \delta \tilde{\mathbf{x}}^{(k)}+v \sigma^{(k)} \mathbf{H}^{(k)} e^{\frac{-2 \pi \mathrm{i}}{M}} \delta \tilde{\mathbf{x}}^{(k-1)}\left(t-\tau^{(k)}\right) \\
\Leftrightarrow e^{\frac{2 k \pi \mathrm{i}}{M}} \delta \dot{\tilde{\mathbf{x}}}^{(k)} & =D \mathbf{F}^{(k)}\left(\mathbf{x}_{s}^{(k)}\right) e^{\frac{2 k \pi \mathrm{i}}{M}} \delta \tilde{\mathbf{x}}^{(k)}+v \sigma^{(k)} \mathbf{H}^{(k)} e^{\frac{2(k-1) \pi \mathrm{i}}{M}} \delta \tilde{\mathbf{x}}^{(k-1)}\left(t-\tau^{(k)}\right) .
\end{aligned}
$$

Applying the transformation $\delta \tilde{\mathbf{x}}^{(k)} \rightarrow \exp (-2 k \pi \mathrm{i} / M) \delta \tilde{\mathbf{x}}^{(k)}$ recovers the original Eq. (5.15) and leaves the Lyapunov spectrum unchanged. Consequently, the MSE is invariant with 
respect to rotations $v \rightarrow \exp (-2 \pi \mathrm{i} / M) v$ meaning that it belongs to the symmetry group $D_{M}$, i.e., the dihedral group of order $2 M$.

As an example Fig. 5.2 shows the MSF for group synchrony for the SNIPER model in the oscillatory regime (for details on the model see Sec. 4.1.2) for $M=1,2,3,4$ in panels (a), (b), (c), and (d), respectively, for a delay time $\tau^{(k)} \equiv \tau=3.5$. Clearly, the discrete rotational invariance shows. Panels (e), (f), (g), and (h) show the to (a), (b), (c), and (d), respectively, corresponding MSF but for a larger delay time of $\tau^{(k)} \equiv \tau=60$. In this case, the stable region coincides with the unit circle as expected for oscillatory systems in the limit of large delay and predicted by Flunkert et al. [2010].

\subsubsection{Spectrum of the coupling matrix}

Not only the MSF but also the spectrum of the coupling matrix are characterized by this discrete rotational symmetry. In the following, we investigate the eigenvalue spectrum of the coupling matrix

$$
\mathbf{G}=\begin{array}{ccccc}
0 & \cdots & \cdots & 0 & \mathbf{A}^{(0)} \\
\mathbf{A}^{(1)} & 0 & \cdots & \cdots & 0 \\
0 & \mathbf{A}^{(2)} & 0 & \cdots & 0 \\
0 & \ddots & \ddots & \ddots & 0 \\
0 & \cdots & 0 & \mathbf{A}^{(M-1)} & 0
\end{array} .
$$

In the case that all $\mathbf{A}^{(k)}$ have maximum $\operatorname{rank} \min \left(N_{k}, N_{k-1}\right)$, there are exactly

$$
n_{0, \max }={ }_{k}\left|N_{k}-N_{k-1}\right|
$$

eigenvalues of $\mathbf{G}$ equal to zero. In general, the exact number of zeros is given by

$$
n_{0}={ }_{k} \max \left(N_{k}, N_{k-1}\right)-\operatorname{rank} \mathbf{A}^{(k)},
$$

which may be larger than $n_{0, \max }$ due to the particular structure of the matrices $\mathbf{A}^{(k)}$, which may have non-maximum rank themselves.

To derive the spectrum of $\mathbf{G}^{M}$, we consider the matrix $\mathbf{G}^{M}$, which is of block diagonal 
structure

$$
\mathbf{G}^{M}=\begin{array}{cccc}
\mathbf{A}^{(0)} \mathbf{A}^{(M)} \mathbf{A}^{(M-1)} \cdots \mathbf{A}^{(1)} & 0 & \cdots & 0 \\
0 & \mathbf{A}^{(1)} \mathbf{A}^{(0)} \mathbf{A}^{(M)} \mathbf{A}^{(M-1)} \cdots \mathbf{A}^{(2)} & \ddots & 0 \\
0 & \ddots & \ddots & 0 \\
0 & \cdots & 0 & \mathbf{A}^{(M)} \mathbf{A}^{(M-1)} \cdots \mathbf{A}^{(0)}
\end{array}
$$

Each block on the diagonal is a cyclic permutation of the first block.

Recall that we assumed that $N_{0} \leq N_{k}(k=1, \ldots, M-1)$, i.e., the zeroth group consists of the least nodes. Furthermore, we assume that each $\mathbf{A}^{(k)}$ has maximum $\operatorname{rank} \min \left(N_{k}, N_{k-1}\right)$. The latter assumption simplifies the argument regarding the zero eigenvalues but the final result is valid for arbitrary ranks of the block matrices. Given the above assumptions, of the blocks in $\mathbf{G}^{M}$, the first block $\left(\mathbf{G}^{M}\right)_{00}=\mathbf{A}^{(0)} \mathbf{A}^{(M)} \mathbf{A}^{(M-1)} \cdots \mathbf{A}^{(1)}$ has lowest rank, since it is an $N_{0} \times N_{0}$ matrix.

The non-zero eigenvalues of a matrix product are invariant against cyclic permutations of the factors. Their number (including degeneracy) equals the rank of the product with lowest rank, i.e., $\left(\mathbf{G}^{M}\right)_{00}$ in our case. This can be easily seen by the following argument: Assume that $\lambda \neq 0$ is an eigenvalue of the first block to the eigenvector $\mathbf{v}$, i.e.,

$$
\mathbf{A}^{(0)} \mathbf{A}^{(M)} \mathbf{A}^{(M-1)} \cdots \mathbf{A}^{(2)} \mathbf{A}^{(1)} \mathbf{v}=\lambda \mathbf{v} .
$$

Multiplying $\mathbf{A}^{(1)}$ from the left yields

$$
\mathbf{A}^{(1)} \mathbf{A}^{(0)} \mathbf{A}^{(M)} \mathbf{A}^{(M-1)} \cdots \mathbf{A}^{(2)}\left(\mathbf{A}^{(1)} \mathbf{v}\right)=\lambda\left(\mathbf{A}^{(1)} \mathbf{v}\right) .
$$

meaning that $\lambda$ is also an eigenvalue of the second block to the eigenvector $\mathbf{A}^{(1)} \mathbf{v} . \mathbf{A}^{(1)} \mathbf{v}$ cannot be equal to the zero vector because this would imply, according to Eq. (5.26), $\lambda=0$ which contradicts the assumption $\lambda \neq 0$. In the same way it can successively be shown that $\lambda$ is an eigenvalue of all non-zero blocks of $\mathbf{G}^{M}$. As a consequence, the nonzero eigenvalues of $\mathbf{G}^{M}$ are given by the non-zero eigenvalues $\left\{\lambda_{0}, \ldots, \lambda_{N_{0}-1}\right\}$ of $\left(\mathbf{G}^{M}\right)_{00}$. As there are $M$ blocks yielding exactly these eigenvalues, each of them is $M$-fold degenerated. In particular, since the row sum of $\mathbf{G}^{M}$ is unity, there is an $M$-fold unity eigenvalue.

The non-zero eigenvalues of $\mathbf{G}$ are then given by the $M$-th roots of the non-zero eigen- 
values of $\mathbf{G}^{M}$, and the spectrum of $\mathbf{G}$ reads

$$
\begin{array}{r}
\left\{v_{j}\right\}_{j=0, \ldots, N-1}=\underset{n_{0}}{\{0, \ldots, 0\} \cup{ }_{k=0}^{M-1}\left\{{ }^{M} \overline{\left|\lambda_{1}\right|} e^{\left[\arg \left(\lambda_{1}\right)+2 \pi k\right] \mathrm{i} / M}, \ldots\right.} \\
\left.\ldots,{ }^{M} \overline{\left|\lambda_{M-1]}\right|} e^{\left[\arg \left(\lambda_{M}\right)+2 \pi k\right] \mathrm{i} / M}\right\},
\end{array}
$$

where $N={ }_{k} N_{k}$, i.e., $N$ is the total number of nodes in the network. In particular, the eigenvalue $\lambda=1$ of $\mathbf{G}^{M}$ corresponds to the $M$ longitudinal eigenvalues $v_{k}=\exp (2 \pi \mathrm{i} k / M)$ of $\mathbf{G}$, which are related to directions longitudinal to the group synchronization manifold. Their existence can already be seen solely by looking at $\mathbf{G}$ itself. Due to the unity-rowsum condition (see Sec. 3.1) $M$ eigenvectors

$$
\begin{aligned}
& 1 \\
& \vdots \quad N_{0} \\
& 1 \\
& \exp (-2 \pi \mathrm{i} k / M) \\
& \mathbf{v}_{k}=\quad \begin{array}{ll}
\vdots & N_{1} \\
\exp (-2 \pi \mathrm{i} k / M) &
\end{array} \\
& \exp (-2 \pi(M-1) \mathrm{i} k / M) \\
& \vdots \quad N_{M-1} \\
& \exp (-2 \pi(M-1) \mathrm{i} k / M)
\end{aligned}
$$

exist which are the eigenvectors to the longitudinal eigenvalue $v_{k}=\exp (2 \pi \mathrm{i} k / M)$. These eigenvalues do not depend on the particular choice the blocks $\mathbf{A}^{(k)}$.

In summary, both the MSF and the spectrum of $\mathbf{G}$ are invariant with respect to rotations $v \rightarrow \exp (2 \pi \mathrm{i} / M) v$. Thus, the evaluation of the MSF can be restricted to the eigenvalues

$$
\left\{{ }^{M} \overline{\left|\lambda_{1}\right|} \exp \left[\operatorname{iarg}\left(\lambda_{1}\right) / M\right], \ldots,{ }^{M} \overline{\left|\lambda_{M}\right|} \exp \left[\operatorname{iarg}\left(\lambda_{M}\right) / M\right], 0\right\},
$$

which all lie inside the angular sector $\arg (v) \in[0,2 \pi / M)$. 


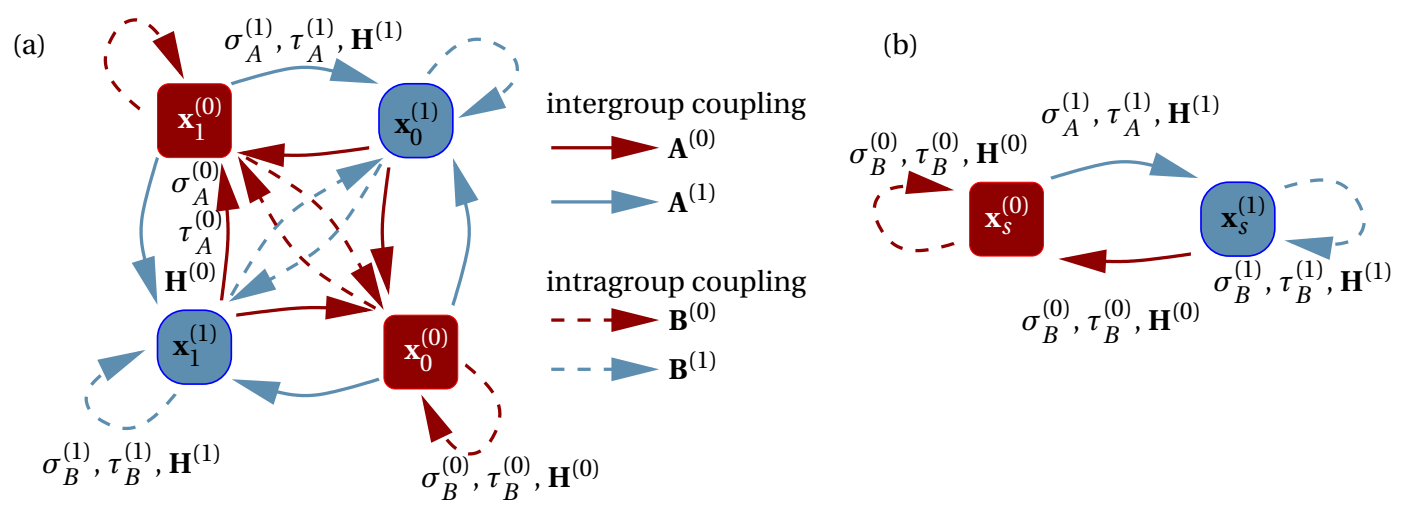

Figure 5.3.: (a) Schematic diagram of two groups visualizing parameters and dynamical variables as in Eq. (5.30) for multiple commuting coupling matrices. Solid arrows denote coupling between the groups; dashed arrows correspond to coupling of nodes in the same group. (b) The corresponding synchronization manifold according to Eq. (5.31). Figure modified from [Dahms et al., 2012].

\subsubsection{Beyond multipartite topologies}

So far we considered multipartite topologies meaning that the nodes of one group couple only to nodes of one other group. This corresponds to a unidirectional topology on the level of groups and, therefore, decouples with the help of the MSF to a network motif of $M$ nodes coupled in a unidirectional ring. The limitation of multipartite networks can be overcome with the help of commuting matrices [Dahms et al., 2012]. In the following, we derive an MSF for group synchrony with two commuting coupling matrices. The restriction to two coupling matrices is for notational convenience, the generalization to multiple matrices is straightforward if those matrices commute.

Introducing the second coupling matrix Eq. (5.2) is generalized, and the dynamics of $M$ groups is given by

$$
\dot{\mathbf{x}}_{i}^{(k)}=\mathbf{F}^{(k)}\left[\mathbf{x}_{i}^{(k)}(t)\right]+\sigma_{A}^{(k)}{ }_{j=0}^{N_{k-1}-1} A_{i j}^{(k)} \mathbf{H}^{(k)} \mathbf{x}_{j}^{(k-1)}\left(t-\tau_{A}^{(k)}\right)+\sigma_{B}^{(k)}{\underset{j=0}{N_{n_{k}}-1}}_{i j}^{(k)} \mathbf{H}^{(k)} \mathbf{x}_{j}^{\left(n_{k}\right)}\left(t-\tau_{B}^{(k)}\right),
$$

where the matrix $\mathbf{A}^{(k)}$ describes the coupling from the $(k-1)$ th to the $k$ th group as before and $\mathbf{B}^{(k)}$ describes the coupling from the $n_{k}$ th to the $k$ th group. That is, the $k$ th group now receives input from two groups, $k-1$ and $n_{k}$. The row sums of all matrices $\mathbf{A}^{(k)}$ 
and $\mathbf{B}^{(k)}$ must be unity. Any constant non-zero row sum can be rescaled by means of the coupling strengths. For simplicity, it is assumed that coupling schemes are identical for both coupling terms.

From Eq. (5.30) with the condition $\mathbf{x}_{0}^{(k)}=\mathbf{x}_{1}^{(k)}=\ldots=\mathbf{x}_{N_{k}-1}^{(k)} \equiv \mathbf{x}_{s}^{(k)}, k=0, \ldots, M-1$, the synchronization manifold is obtained as

$$
\dot{\mathbf{x}}_{s}^{(k)}=\mathbf{F}^{(k)}\left[\mathbf{x}_{s}^{(k)}(t)\right]+\sigma_{A}^{(k)} \mathbf{H}^{(k)} \mathbf{x}_{s}^{(k-1)}\left(t-\tau_{A}^{(k)}\right)+\sigma_{B}^{(k)} \mathbf{H}^{(k)} \mathbf{x}_{s}^{\left(n_{k}\right)}\left(t-\tau_{B}^{(k)}\right)
$$

for $k=0, \ldots, M-1$. Note that the sum of $\sigma_{A}^{(k)}$ and $\sigma_{B}^{(k)}$ must yield the overall coupling strength $\sigma^{(k)}$ used before in order to get the same dynamical regime in the synchronization manifold as in the case of one coupling matrix, see Eq. (5.6), i.e., $\sigma_{A}^{(k)}+\sigma_{B}^{(k)}=$ $\sigma^{(k)}$.

Figure 5.3(a) shows schematically the coupling parameters and matrices that are present in Eq. (5.30). In the case of two groups shown here, $\mathbf{B}^{(0)}$ and $\mathbf{B}^{(1)}$ represent the coupling within the groups, depicted by dashed arrows. Panel (b) is a schematic diagram of the synchronization manifold given by Eq. (5.31). In the synchronization manifold, the coupling within a group translates into a self-feedback loop, which is depicted by dashed arrows.

Let $\mathbf{G}_{A}$ be the matrix containing the blocks $\mathbf{A}^{(k)}$ at positions $(k, k-1)$ and $\mathbf{G}_{B}$ the matrix containing the blocks $\mathbf{B}^{(k)}$ at positions $\left(k, n_{k}\right)$ where $n_{0}, \ldots, n_{M-1}$ is an arbitrary permutation of the integers $0, \ldots, M-1$. If $\mathbf{G}_{A}$ and $\mathbf{G}_{B}$ commute, i.e., $\left[\mathbf{G}_{A}, \mathbf{G}_{B}\right]=0$, they have a common set of eigenvectors and, thus, can be block diagonalized simultaneously, i.e., by the same transformation. By this block diagonalization an MSE is obtained:

$$
\delta \dot{\mathbf{x}}^{(k)}=\mathbf{D} \mathbf{F}^{(k)}\left(\mathbf{x}_{s}^{(k)}\right) \delta \overline{\mathbf{x}}^{(k)}(t)+\sigma_{A}^{(k)} v_{A} \mathbf{H}^{(k)} \delta \overline{\mathbf{x}}^{(k-1)}\left(t-\tau_{A}^{(k)}\right)+\sigma_{B}^{(k)} v_{B} \mathbf{H}^{(k)} \delta \overline{\mathbf{x}}^{\left(n_{k}\right)}\left(t-\tau_{B}^{(k)}\right)
$$

for $k=0, \ldots, M-1$, where $v_{A}$ and $v_{B}$ are chosen from the eigenvalue spectrum of the matrices $\mathbf{G}_{A}$ and $\mathbf{G}_{B}$, respectively. Thus, the MSF is now calculated in the four-dimensional space spanned by $\operatorname{Re} v_{A}, \operatorname{Im} v_{A}, \operatorname{Re} v_{B}$, and $\operatorname{Im} v_{B} . v_{A}$ and $v_{B}$ are the eigenvalues to the same, common eigenvector of $\mathbf{G}_{A}$ and $\mathbf{G}_{B}$. Thus, in order to evaluate the stability of synchrony in a given network, the eigenvalues of the matrices $\mathbf{G}_{A}$ and $\mathbf{G}_{B}$ representing this network have to be evaluated in pairs corresponding to one of the common eigenvectors of $\mathbf{G}_{A}$ and $\mathbf{G}_{B}$. 


\section{Example: Neural networks}

As discussed before, the MSF approach has successfully been applied to study synchronization in neural networks independently of a specific network topology [Dhamala et al., 2004; Jirsa, 2008; Lehnert et al., 2011a]. The MSF for group synchrony and, in particular, the framework of commuting matrices can be used to describe more complex synchronization patterns in neural networks like the coexistence of oscillations with different frequencies [Bazhenov and Timofeev, 2006]. In addition, it allows for taking into account biological details, which have to be neglected when using the standard MSF. For example, the brain is organized in different brain areas leading to different delay times between neurons of different areas and neurons within the same area, which can be modeled by two commuting coupling matrices. Furthermore, different types of neurons exist, corresponding to different local dynamics which is, as discussed above, easily treated with the MSF for group synchrony.

Here we discuss the stability of group synchrony in a network of FitzHugh-Nagumo systems (for details on the model see Sec. 4.1.4). For simplicity, we consider two groups and assume that the local dynamics of all nodes are identical. However, we choose different delay times for the inter- and the intra- group coupling to mimic two groups from different brain areas. The inter-group coupling is given by

$$
\mathbf{G}_{A}=\begin{array}{cc}
0 & \mathbf{A}^{(0)} \\
\mathbf{A}^{(1)} & 0
\end{array},
$$

where the matrices $\mathbf{A}^{(1)}$ and $\mathbf{A}^{(2)}$ describe the coupling from the second to the first group and vice versa, respectively; see also Sorrentino and Ott [2007]. The second coupling matrix reads

$$
\mathbf{G}_{B}=\begin{array}{cc}
\mathbf{B}^{(0)} & 0 \\
0 & \mathbf{B}^{(1)}
\end{array}
$$

i.e., $n_{0}=0$ and $n_{1}=1$ in Eq. (5.30), meaning that $\mathbf{B}^{(k)}$ accounts for the coupling between the nodes inside the $k$ th group.

Using the forms (5.33) and (5.34), the commutation relation $\left[\mathbf{G}_{A}, \mathbf{G}_{B}\right]=0$ is equivalent to

$$
\begin{aligned}
\mathbf{A}^{(0)} \mathbf{B}^{(1)} & =\mathbf{B}^{(0)} \mathbf{A}^{(0)} \\
\mathbf{A}^{(1)} \mathbf{B}^{(0)} & =\mathbf{B}^{(0)} \mathbf{A}^{(1)} ;
\end{aligned}
$$

see also [Dahms, 2011]. 

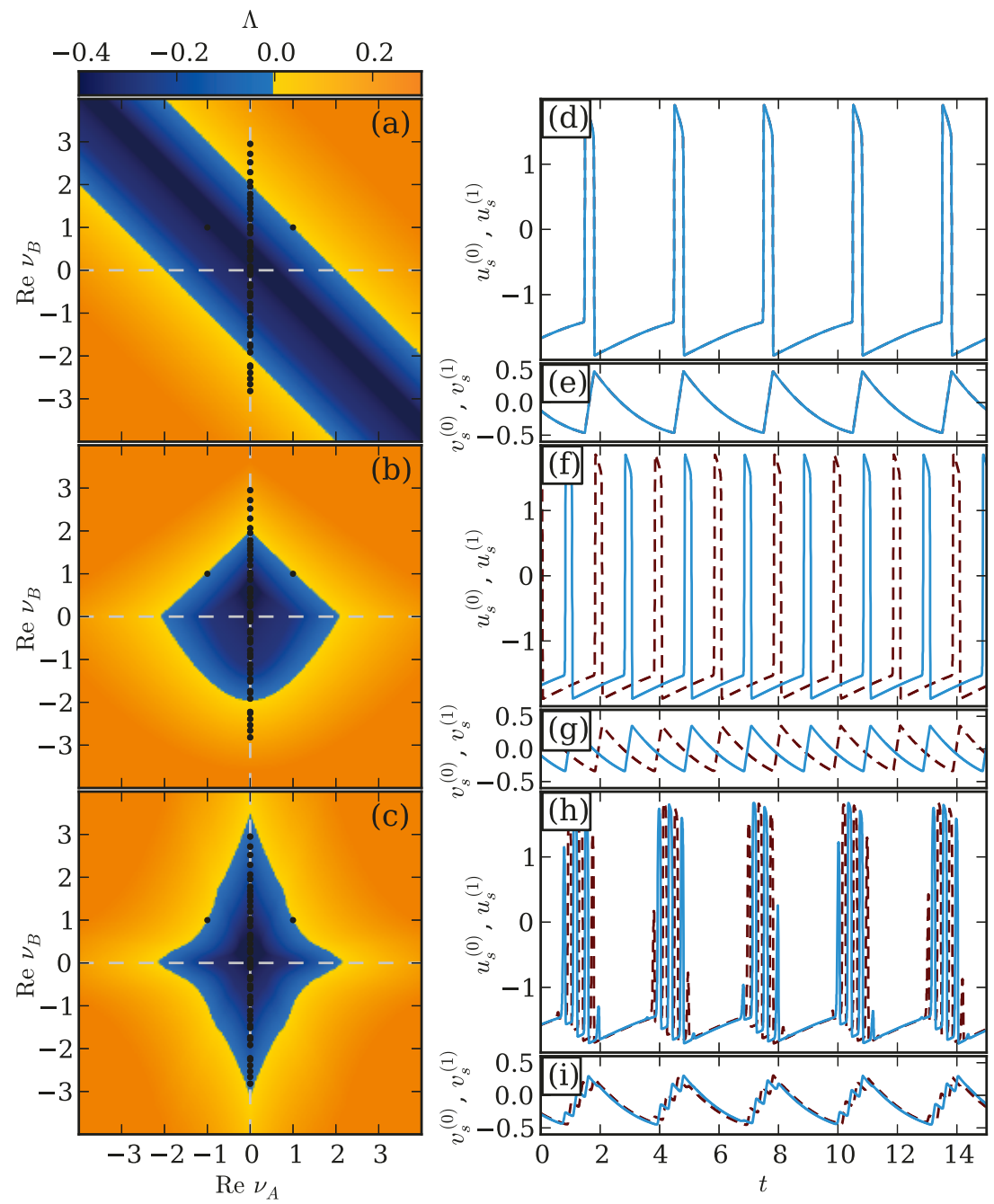

Figure 5.4.: (a)-(c): Master stability function (MSF) for networks of FitzHugh-Nagumo oscillators governed by Eq. (5.36) in the $\left(\operatorname{Re} v_{A}, \operatorname{Re} v_{B}\right)$ plane for $\operatorname{Im} v_{A}=\operatorname{Im} v_{B}=0$ and different delay times. The black dots denote the location of the eigenvalue pairs for the example topology (5.38). (d)-(i): Time series of the dynamics in the first (red) and second (blue) group. Parameters: (a),(d),(e): zero-lag synchronization $\left(\tau_{B}^{(k)}=3\right.$ ), (b), $(f),(g)$ : anti-phase synchronization $\left(\tau_{B}^{(k)}=2\right),(c),(h),(i)$ : synchronized bursting $\left(\tau_{B}^{(k)}=3.2\right.$ ). Other parameters: $\sigma_{A}^{(k)}=\sigma_{B}^{(k)}=0.5, \tau_{A}^{(k)}=3, \epsilon=0.01, a=1.3$ (groups $k=0,1)$. Figure from [Dahms et al., 2012]. 
These conditions are fulfilled for certain classes of matrices only.

The local dynamics of the $i$ th node in the $k$ th cluster are given by

$$
\mathbf{F}\left(\mathbf{x}_{i}^{(k)}\right)=\frac{\frac{1}{\epsilon}\left(u_{i}^{(k)}-\frac{1}{3} u_{i}^{(k)^{3}}-v_{i}^{(k)}\right)}{u_{i}^{(k)}+a} \quad+\left(\sigma_{A}^{(k)}+\sigma_{B}^{(k)}\right) \mathbf{H} \mathbf{x}_{i}^{(k)},
$$

with $\mathbf{x}_{i}^{(k)}=\left(u_{i}^{(k)}, v_{i}^{(k)}\right)$ and $k=0,1$, cf. Eq. (4.8). Recall that $u$ and $v$ denote the activator and inhibitor variables, respectively. The parameter $a$ determines the threshold of excitability. As in Chapter 4, we will consider the FitzHugh-Nagumo model in the excitable regime ( $a=1.3$ ) and the time-scale parameter $\epsilon$ is chosen as $\epsilon=0.01$. The synchronized dynamics and the MSE are then given by Eq. (5.31) and Eq. (5.32), respectively. The coupling scheme is given by

$$
\mathbf{H}^{(0)}=\mathbf{H}^{(1)} \equiv \mathbf{H}=\begin{array}{cc}
1 / \epsilon & 0 \\
0 & 0
\end{array} .
$$

The synchronized dynamics given by Eq. (5.31) is equivalent to a system of two coupled nodes with self-feedback. In [Schöll et al., 2009; Panchuk et al., 2013] it was shown that depending on the delay times, the coupling strength, and the strength of the selffeedback different dynamical scenarios, i.e., in-phase synchronization, anti-phase synchronization, or bursting can arise. Figure 5.4 shows the MSF in panels (a)-(c) for inphase synchronization, anti-phase synchronization and for synchronization in two bursting groups, respectively. The right hand panels of Fig. 5.4 depict the corresponding time series: In panels (d), (f), and (h) for the activator and in panels (e), (g), and (i) for the inhibitor for in-phase, anti-phase, and bursting dynamics, respectively. Obviously, the different synchronization scenarios yield very different MSFs. Thus, some topologies might show stable synchronization for one of the patterns but not for the others.

However, for all scenarios the stable region contains the unity square, i.e., $\left(\operatorname{Re} v_{A}, \operatorname{Re} v_{B}\right) \in$ $[-1,1] \times[-1,1]$. With Gershgorin's circle theorem [Gerschgorin, 1931; Earl and Strogatz, 2003] it can easily be shown that the eigenvalues of symmetric matrices with positive entries and unity-row sum are always contained in the interval $[-1,1]$. Recall that in general (in the sense of not necessarily symmetric matrices) the eigenvalue of matrices with positive entries and unity-row sum are contained in the unit circle in the $(\operatorname{Re} v, \operatorname{Im} v)$ plane (see Sec 4.1.2). In consequence, synchronization is stable for all three dynamics presented here if $\mathbf{G}_{A}$ and $\mathbf{G}_{B}$ have only positive entries, i.e., if the coupling is excitatory. 
Thus, only the introduction of inhibitory links can lead to desynchronization as it was already discussed in Sec. 4.2.2 where the case $\sigma_{A}^{(k)}=\sigma_{B}^{(k)}=\sigma$ and $\tau_{A}^{(k)}=\tau_{B}^{(k)}=\tau$ was considered; see also [Lehnert et al., 2011a].

In the following, we consider a network of two groups with inhibitory links which will exhibit stable synchronization only in one of the synchronization patterns discussed above but not in the other ones. We assume that both groups are of equal size: $N_{0}=N_{1} \equiv$ $\bar{N}=100$. The nodes inside a group are assigned to be all-to-all coupled including selfcoupling, i.e., $\mathbf{A}^{(0)}=\mathbf{A}^{(1)} \equiv \mathbf{A}$ with $A_{i j}=1 / \bar{N}, i, j=0, \ldots, \bar{N}-1$. For the links between the groups, we choose $\mathbf{B}^{(0)}=\mathbf{B}^{(1)} \equiv \mathbf{B}$ where $\mathbf{B}$ is an undirected $\bar{N} \times \bar{N}$ random matrix with both excitatory (positive entries) and inhibitory links (negative entries). Then, the coupling matrices read

$$
\mathbf{G}_{A}=\begin{array}{cc}
0 & \mathbf{A} \\
\mathbf{A} & 0
\end{array}, \quad \mathbf{G}_{B}=\begin{array}{cc}
\mathbf{B} & 0 \\
0 & \mathbf{B}
\end{array} .
$$

In this case, the commuting condition given by (5.35) simplifies to

$$
\mathbf{A B}=\mathbf{B A} .
$$

The matrix $\mathbf{B}$ is constructed to have a fixed node degree with 12 excitatory and 9 inhibitory links for each node. Then, $\mathbf{A}$ and $\mathbf{B}$ and, thus, $\mathbf{G}_{A}$ and $\mathbf{G}_{B}$ commute. The black dots in Fig. 5.4 denote the corresponding eigenvalue pairs. In panels (a) and (b) some eigenvalues are located outside the stable region, while in panel (c) they are all inside, which means that the zero-lag and anti-phase synchronized solutions will be unstable in such a network, while synchronization in the bursting state will be stable.

\section{Symmetry of the MSF for commuting coupling matrices}

As discussed in Sec. 5.1.3 the MSF of group synchrony is invariant to transformations of the form $v \rightarrow \exp (2 \pi \mathrm{i} / M) v$ if only one coupling matrix is used. In the case of two commuting matrices and an MSE given by Eq. (5.32), this invariance does not show in general but only in the case of two groups with coupling matrices $\mathbf{G}_{A}$ and $\mathbf{G}_{B}$ such that the non-empty blocks are on different positions, meaning that $\mathbf{G}_{A}$ is block antidiagonal matrix, while $\mathbf{G}_{B}$ is a block diagonal matrix, as it for example the case in Eq. (5.38). In this case the invariance is modified to

$$
\left(v_{A}, v_{B}\right) \rightarrow\left(-v_{A}, v_{B}\right)
$$


This can easily be seen by applying transformation (5.40) to Eq. (5.32) which yields

$$
\delta \dot{\mathbf{x}}^{(k)}=\mathbf{D} \mathbf{F}^{(k)}\left(\mathbf{x}_{s}^{(k)}\right) \delta \overline{\mathbf{x}}^{(k)}(t)-\sigma_{A}^{(k)} v_{A} \mathbf{H}^{(k)} \delta \overline{\mathbf{x}}^{(k-1)}\left(t-\tau_{A}^{(k)}\right)+\sigma_{B}^{(k)} v_{B} \mathbf{H}^{(k)} \delta \overline{\mathbf{x}}^{\left(n_{k}\right)}\left(t-\tau_{B}^{(k)}\right) .
$$

If we now multiply this equation by $\exp (-k \pi i)$ and transform the perturbations according to $\delta \tilde{\mathbf{x}}^{(k)} \rightarrow \exp (-k \pi i) \delta \tilde{\mathbf{x}}^{(k)}$ we recover the original Eq. (5.43) and, thus, confirm its invariance with respect to transformation (5.40).

In Fig. 5.4 (b) and (c) symmetry (5.40) shows clearly. However, in panel (a) the symmetry appears to be broken. This is because in panel (a) both groups synchronize to $\mathbf{x}_{s}^{(0)}=\mathbf{x}_{s}^{(1)}$ and, thus, strictly speaking not group but zero-lag synchrony is observed meaning that a transformation of the form $\delta \tilde{\mathbf{x}}^{(k)} \rightarrow \exp (-k \pi \mathrm{i}) \delta \tilde{\mathbf{x}}^{(k)}$, i.e., $\delta \tilde{\mathbf{x}}^{(0)} \rightarrow \delta \tilde{\mathbf{x}}^{(0)}$ and $\delta \tilde{\mathbf{x}}^{(1)} \rightarrow \delta \tilde{\mathbf{x}}^{(1)}$, is not valid, since $\delta \tilde{\mathbf{x}}^{(0)}=\delta \tilde{\mathbf{x}}^{(1)}$. Instead, we find for the case of zero-lag synchrony an invariance of the form

$$
\left(v_{A}, v_{B}\right) \rightarrow\left(v_{A}+\Delta v, v_{B}-\Delta v\right),
$$

with $\Delta v \in \mathbb{C}$ meaning that the Lyapunov exponent $\Lambda$ depends only on the sum $v_{A}+v_{B}$ (see Fig. 5.4 (a)). This follows directly from Eq. (5.32) by substituting $\delta \tilde{\mathbf{x}}^{(k)} \equiv \delta \tilde{\mathbf{x}}, \mathbf{H}^{(k)} \equiv \mathbf{H}$, $\sigma_{A}^{(k)}=\sigma_{B}^{(k)} \equiv \sigma$ and $\tau_{A}^{(k)}=\tau_{B}^{(k)} \equiv \tau, k=0,1$, which reduces Eq. (5.32) to

$$
\delta \dot{\overline{\mathbf{x}}}=\mathbf{D F}\left(\mathbf{x}_{s}\right) \delta \overline{\mathbf{x}}(t)+\sigma\left(v_{A}+v_{B}\right) \mathbf{H} \delta \overline{\mathbf{x}}(t-\tau),
$$

which only depends on the sum $v_{A}+v_{B}$ but not on the individual parameters $v_{A}$ and $v_{B}$.

\subsection{THE STUART-LANDAU OSCILLATOR:}

\section{AN ANALYTICALLY TRACTABLE EXAMPLE}

In Sec. 5.2, the MSF for group and cluster synchronization has been derived. In general, the MSE, given by Eq. (5.15), has to be solved numerically since the Jacobian $D \mathbf{F}\left(\mathbf{x}_{s}\right)$ depends on the synchronized state $\mathbf{x}_{s}$ and is, thus, in the majority of the cases time dependent. However, if the Jacobian is time-independent, as it is the case for Stuart-Landau oscillator, an analytic treatment is possible [Choe et al., 2010; Dahms, 2011; Choe et al., 2011]. As this stability analysis is the basis of the further investigations on the dynamics of coupled Stuart-Landau oscillators (see Sec. 6.2 and Chapters 9 and 10), I will give here a short recapitulation on the results obtained in [Choe et al., 2010; Dahms, 2011; Choe et al., 2011]. 


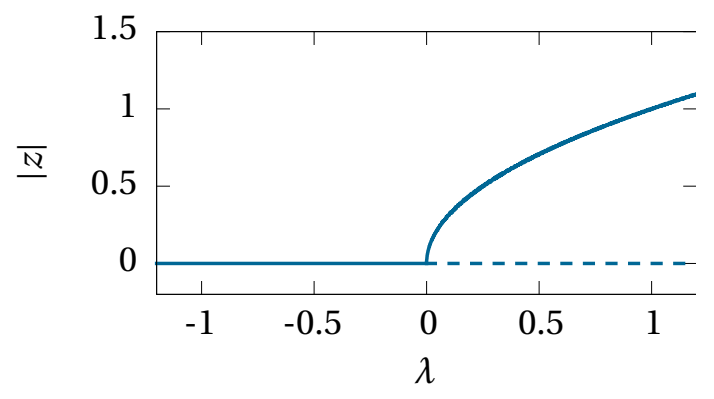

Figure 5.5.: Supercritical Hopf bifurcation. Radius of oscillations vs. the bifurcation parameter $\lambda$. Solid and dashed lines mark stable and unstable states, respectively.

The dynamics of the Stuart-Landau oscillator is given by

$$
\dot{z}=\lambda+\mathrm{i} \omega-(1+i \gamma)|z|^{2} \quad z
$$

with the complex variable $z \in \mathbb{C}$. It arises in a center manifold expansion close to a Hopf bifurcation with $\lambda$ as the bifurcation parameter. For $\lambda<0$, the system exhibits a stable focus. At the bifurcation point $\lambda=0$, the focus loses stability, and a stable limit cycle with radius $\sqrt{\lambda}$ is born. For a bifurcation diagram see Fig. 5.5. The parameters $\omega$ and $\gamma$ denote the intrinsic rotation frequency while approaching the fixed point at the origin and the frequency detuning for finite oscillation amplitudes, respectively. In the following, I will consider $\gamma=0$, in which case the frequency of the focus or the limit cycle, respectively, is given by $\omega$.

Consider now a system of $N$ delay-coupled Stuart-Landau oscillators $z_{j}, j=0, \ldots, N-$ 1:

$$
\dot{z}_{j}=\left[\lambda+\mathrm{i} \omega-\left|z_{j}\right|^{2}\right] z_{j}+\sigma_{n=0}^{N-1} G_{j n}(t)\left[z_{n, \tau}-z_{j}(t)\right]
$$

where $\sigma=K e^{\mathrm{i} \beta}$ is the complex, overall coupling strength, and $\tau$ is the delay time. In the following, I denote delayed variables by an index $\tau$, e.g., $z_{n, \tau} \equiv z_{n}(t-\tau)$. The real-valued coupling matrix $\mathbf{G}=\left\{G_{j n}(t)\right\}_{j, n=0, \ldots, N-1}$ determines the topology of the network.

Equation (5.45) can be rewritten in amplitude and phase variables with $r_{j}=\left|z_{j}\right|$ and 
$\varphi_{j}=\arg \left(z_{j}\right):$

$$
\begin{aligned}
\dot{r}_{j}(t) & =\lambda-r_{j}^{2} r_{j}+K{ }_{n=0}^{N-1} G_{j n} r_{n, \tau} \cos \beta+\varphi_{n, \tau}-\varphi_{j}-r_{j} \cos \beta, \\
\dot{\varphi}_{j}(t)= & \omega+K{ }_{n=0}^{N-1} G_{j n} \frac{r_{n, \tau}}{r_{j}} \sin \beta+\varphi_{n, \tau}-\varphi_{j}-\sin \beta .
\end{aligned}
$$

In a cluster state, $r_{j}$ and $\varphi_{j}$ follow

$$
\begin{aligned}
r_{j} & =r_{0, m}, \\
\varphi_{j} & =\Omega_{m} t+\frac{2 \pi m}{N} j,
\end{aligned}
$$

with constant $r_{0, m}$ and $\Omega_{m} . m=0$ correspond to zero-lag synchronization, i.e., one cluster arises. For $m>0$, the number of clusters $M$ can be calculated as $M=1 \mathrm{~cm}(m, N) / m$, where lcm stands for the least common multiple. Thus, depending on $N$ and $M$, several states might exist which are characterized by the same number of clusters. In the following I will refer to all of these different states by the collective term $M$-cluster state if it is not necessary to distinguish between the different $m$-states.

Figure 5.6 shows a schematic view of all cluster states described by Eq. (5.47) in a unidirectional ring configuration of four nodes. In panel (a), the nodes are in zero-lag synchronization. Panels (b) and (d) show two different splay states, i.e., $M=N=4$, where the phase difference between subsequent nodes in panel (b) is $\pi / 2$, i.e., $m=1$, and in panel (d) $3 \pi / 2$, i.e., $m=3$. Thus, the latter case can be considered as a reversed splay state, where the ordering of the nodes is given by their position in the unidirectional ring. Panel (b) depicts an anti-synchronized state, i.e., a 2-cluster state.

Substituting Eq. (5.47) into Eq. (5.46) yields a transcendental equation for the common radius $r_{0, m}$ and the common frequency $\Omega_{m}$ in an $m$-state:

$$
\begin{aligned}
r_{0, m}^{2} & =\lambda-K \cos \beta+K{ }_{n=0}^{N-1} G_{j n} \cos \left[\beta-\Omega_{m} \tau+2 \pi m(n-j) / N\right], \\
\Omega_{m} & =\omega-K \sin \beta+K{ }_{n=0}^{N-1} G_{j n} \sin \left[\beta-\Omega_{m} \tau+2 \pi m(n-j) / N\right] .
\end{aligned}
$$

Note that Eq. (5.48) depends on $j$ such that a solution with a common amplitude and 
(a)

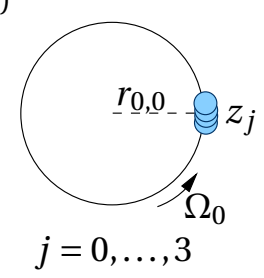

(b)

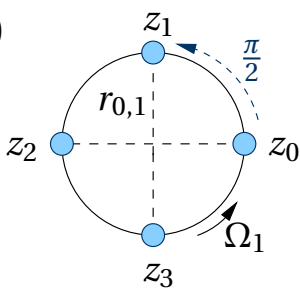

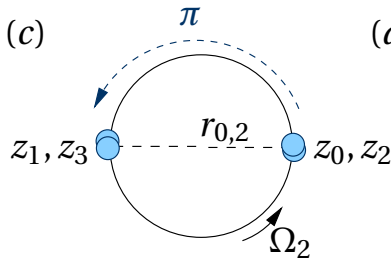

(c)

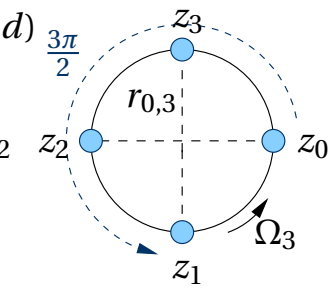

Figure 5.6.: Schematic view of (a) zero-lag synchronization ( $m=0, M=1)$, (b) the splay state ( $m=1, M=4)$, (c) anti-synchronization ( $m=2, M=2)$, and (d) the reversed splay state $(m=3, M=4)$. Each cluster consists of the same number of nodes. For convenience, the common radii of the different states are depicted as equal; however, according to Eq. (5.48) the common radius depends on $m$ and thus on the state .

frequency for all $j, j=0, \ldots, N-1$, will only be possible under restrictions regarding the topology G. Topologies for which such a solution can be found include circulant matrices, i.e., matrices with the property $A_{j,(j+i) \bmod N}=A_{0 i}, j, i=0, \ldots, N-1$; see Subsec. 2.3.1. In the remaining part of this recapitulation, the simplest case of a circulant matrix, the unidirectional ring, is investigated, i.e., $G_{i, j}=\delta_{i+1, j}, i, j=0, \ldots, N-1$; see Subsec. 2.3.1.

Choe et al. showed that the Floquet exponent $\Lambda$ transversal to the synchronous manifold can be obtained from the transcendental equation

$$
0=\operatorname{det}\left[\mathbf{J}_{0}-\Lambda \mathbf{I}_{2}+K Q_{l}(\Lambda) \mathbf{R}_{m}\right]
$$

where $Q_{l}(\Lambda)=-1+e^{-\Lambda \tau+2 \mathrm{i} l \pi / N}$,

$\mathbf{J}_{\mathbf{0}}$ is the Jacobian of the local dynamics given by

$$
\mathbf{J}_{0}=\begin{array}{cc}
-2 r_{0, m}^{2} & 0 \\
0 & 0
\end{array}
$$

and the matrix $\mathbf{R}_{m}$ reads

$$
\mathbf{R}_{m}=\begin{array}{cc}
\cos \Phi_{m} & -\sin \Phi_{m} \\
\sin \Phi_{m} & \cos \Phi_{m}
\end{array}
$$

with the abbreviation $\Phi_{m}=\beta-\Omega_{m} \tau+2 \pi m / N$.

Note that the Floquet exponent $\Lambda$ is a complex variable in contrast to the real-valued Lyapunov exponent used in Sec. 5.1 and Chapter 4. For the Floquet exponent, stability 
is characterized by its real part. Equation (5.49) has to be evaluated for $l=1, \ldots, N-$ 1. For $\tau>0$ an infinite number of solutions for the Floquet exponent $\Lambda$ arises. From these solutions, the Floquet exponent with the largest real part determines the stability; if this real part is positive the synchronization is unstable, while its is stable if all Floquet exponents have negative real parts. $l=0$ in Eq. (5.49) corresponds to the longitudinal eigenvalue; see Subsec. 3.2.3.

\subsubsection{Phase of the complex coupling strength}

Choe et al. [2010] identified the phase $\beta$ of the complex coupling strength $\sigma=K \exp (i \beta)$ as a crucial parameter in the control of cluster synchronization. They showed that for

$$
\beta_{m} \equiv \Omega_{m} \tau-2 m \pi / N+2 l \pi, \quad l=0, \pm 1, \pm 2, \ldots,
$$

the dominant Floquet exponent is given by the transcendental equation

$$
\Lambda=K\left(-1+e^{-\Lambda \tau+2 \mathrm{i} l \pi / N}\right) .
$$

Equation (5.53) has only solutions with negative real parts for all values of $\tau, K>0$. Consequently, the choice $\beta=\beta_{m}$ ensures stability and existence of the $m$-state for the whole $(K, \tau)$-plane. However, other stable cluster states may coexist in parts of the $(K, \tau)$-plane as will be discussed in Sec. 9.4.

\subsection{NON-SMOOTH SYSTEMS}

In Section 5.1, I introduced the MSF for cluster and group synchrony in smooth systems. The MSF for zero-lag as well as for group synchronization can be generalized to nonsmooth systems as we have shown in [Ladenbauer et al., 2013]. Non-smooth systems are characterized by a discontinuity in at least of of the variables. They arise in various applications [Bernardo et al., 2008]. Examples include electronic circuits [di Bernardo et al., 1998; Banarjee and Verghese, 2001], hybrid control systems [Ye et al., 1998; Cassandras et al., 2001], and biological networks [Battogtokh et al., 2006; Aihara and Suzuki, 2010; Izhikevich, 2007]. In particular, non-smooth systems are frequently used in neurocscience where neuron models are often of integrate-and-fire type and synaptic couplings are commonly modeled by non-smooth functions. In integrate-and-fire models the discontinuity arises when the voltage is set back to its resting potential after reaching 
a threshold. This type of models is often employed in large-scale computational studies of network activity [Izhikevich and Edelman, 2008; Vogels and Abbott, 2009; LitwinKumar and Doiron, 2012; Destexhe, 2009; Brunel, 2000; Gigante et al., 2007], and forms the hardware elements of neuromorphic systems designed for spike-based, brain-style computations [Indiveri et al., 2006; Jo et al., 2010]. In this Section, the MSF for nonsmooths systems is introduced and its potential is demonstrated by studying synchrony and group states for recurrent networks of adaptive integrate-and-fire models. The section is based on [Ladenbauer et al., 2013].

\subsubsection{Master stability function for non-smooth systems}

In this Subsection, I introduce the MSF for non-smooth systems. Before considering the case of group synchrony, the more simple case of zero-lag synchronization is discussed.

\section{Zero-lag synchrony}

As discussed above non-smooth systems are characterized by a discontinuity. To describe this discontinuity mathematically, we define a scalar-valued differentiable function $\varphi\left(\mathbf{x}_{i}\right)$ where $\mathbf{x}_{i}$ is the state of the $i$ th node. In the following, we assume that $\varphi\left(\mathbf{x}_{i}\right)=0$ indicates the occurrence of a discontinuity. Then, a delay-coupled network of $N$ nonsmooth systems of dimension $d$ can be described by two sets of equations, one for the dynamics of the network elements between the discontinuities,

$$
\dot{\mathbf{x}}_{i}=\mathbf{f}\left(\mathbf{x}_{i}\right)+\sigma{ }_{j=0}^{N-1} A_{i j} \mathbf{H} \mathbf{x}_{j, \tau}, \quad \text { if } \quad \varphi\left(\mathbf{x}_{i}\right) \neq 0,
$$

and one for the jumps,

$$
\mathbf{x}_{i}^{+}=\mathbf{g}\left(\mathbf{x}_{i}\right), \quad \text { if } \quad \varphi\left(\mathbf{x}_{i}\right)=0,
$$

$i=0, \ldots, N-1, \mathbf{x}_{i} \in \mathbb{R}^{d}$ and $\mathbf{x}_{i, \tau} \equiv \mathbf{x}_{i}(t-\tau)$, where $\tau$ is the delay time. We assume that $\mathbf{x}_{i}(t)$ is piecewise continuous and that $\mathbf{f}, \mathbf{g}$ are differentiable vector functions. $\mathbf{f}$ represents the local dynamics, while $\mathbf{g}$ defines the system dynamics when reaching the threshold. The $d \times d$ matrix $\mathbf{H}$ is the coupling scheme describing how node $j$ couples to node $i$. $\varphi$ is a scalar-valued, differentiable function, where $\varphi\left(\mathbf{x}_{i}\right)=0$ indicates the occurrence of a discontinuity, and $\mathbf{x}_{i}^{+}(t) \equiv \lim _{s \backslash t} \mathbf{x}_{i}(s)$ denotes a right-sided limit. $\mathbf{G}$ denotes the coupling matrix and $\sigma$ the overall coupling strength. 
As in the derivation of the standard MSF, we assume that $\mathbf{G}$ obeys the unity-row-sum condition, i.e., $\quad{ }_{j} G_{i j}=1$ for all $i=0, \ldots, N-1$ (see Subsec. 3.1). Then, a zero-lag synchronous state exists and is given by

$$
\dot{\mathbf{x}}_{s}=\mathbf{f}\left(\mathbf{x}_{s}\right)+\sigma \mathbf{H} \mathbf{x}_{s, \tau} \equiv \mathbf{F}\left(\mathbf{x}_{s}, \mathbf{x}_{s, \tau}\right)
$$

We denote the set of times at which $\mathbf{x}_{s}$ jumps by $\left\{t_{s}\right\}$. The abbreviation $\mathbf{F}\left(\mathbf{x}_{s}, \mathbf{x}_{s, \tau}\right)$ is introduced for convenience throughout this section.

From (5.54) a variational equation can be derived

$$
\delta \dot{\mathbf{X}}=\left[\mathbf{I}_{N} \otimes D \mathbf{f}\left(\mathbf{x}_{S}\right)\right] \delta \mathbf{X}+\sigma[\mathbf{G} \otimes \mathbf{H}] \delta \mathbf{X}_{\tau},
$$

where $\delta \mathbf{X}=\left(\mathbf{x}_{0}-\mathbf{x}_{s}, \ldots, \mathbf{x}_{N-1}-\mathbf{x}_{s}\right)$ denotes the deviation from the synchronization manifold. $\otimes$ is the Kronecker product. Equation (5.57) describes the time evolution of the variation if there are no jumps in the synchronous manifold. However, if a discontinuity in $\mathbf{x}_{\mathbf{s}}$ or $\mathbf{x}_{\mathbf{s}, \tau}$ occurs an appropriate linearization condition has to be found. Using first order approximations of the times at which $\varphi\left(\mathbf{x}_{s}+\delta \mathbf{x}_{i}\right)=0$ and using Taylor expansions of $\mathbf{f}\left(\mathbf{x}_{i}\right)+{ }_{j=0}^{N-1} G_{i j} \mathbf{H} \mathbf{x}_{j, \tau}$ around $\mathbf{x}_{s}$ and $\mathbf{x}_{s}^{+}$, at both $t_{s}$ and $t_{s}+\tau$, we obtain

$$
\begin{array}{ll}
\delta \mathbf{X}^{+}=\left[\mathbf{I}_{N} \otimes \mathbf{T}\right] \delta \mathbf{X}, & \text { if } t \in\left\{t_{s}\right\}, \\
\delta \mathbf{X}^{+}=\delta \mathbf{X}+[\mathbf{G} \otimes \mathbf{U}] \delta \mathbf{X}_{\tau}, & \text { if } t-\tau \in\left\{t_{s}\right\}
\end{array}
$$

The matrices $\mathbf{T}$ and $\mathbf{U}$ are given by:

$$
\begin{aligned}
& \mathbf{T} \equiv D \mathbf{g}\left(\mathbf{x}_{s}\right)+\frac{\mathbf{F}\left(\mathbf{x}_{s}^{+}, \mathbf{x}_{s, \tau}\right)-D \mathbf{g}\left(\mathbf{x}_{s}\right) \mathbf{F}\left(\mathbf{x}_{s}, \mathbf{x}_{s, \tau}\right) D \varphi\left(\mathbf{x}_{s}\right)}{D \varphi\left(\mathbf{x}_{s}\right) \mathbf{F}\left(\mathbf{x}_{s}, \mathbf{x}_{s, \tau}\right)}, \\
& \mathbf{U} \equiv \frac{\mathbf{F}\left(\mathbf{x}_{s}, \mathbf{x}_{s, \tau}^{+}\right)-\mathbf{F}\left(\mathbf{x}_{s}, \mathbf{x}_{s, \tau}\right) D \varphi\left(\mathbf{x}_{s, \tau}\right)}{D \varphi\left(\mathbf{x}_{s, \tau}\right) \mathbf{F}\left(\mathbf{x}_{s, \tau}, \mathbf{x}_{s, 2 \tau}\right)} .
\end{aligned}
$$

For the detail of the derivation see the supplements of [Ladenbauer et al., 2013].

Block-diagonalization of Eqs. (5.57) and (5.58) then leads to the MSE [Ladenbauer et al., 2013]

$$
\begin{array}{ll}
\dot{\delta \mathbf{x}}=D \mathbf{f}\left(\mathbf{x}_{s}\right) \delta \mathbf{x}+\sigma v \mathbf{H} \mathbf{x}_{s, \tau} \delta \mathbf{x}_{\tau}, & \text { if } t, t-\tau \notin\left\{t_{s}\right\}, \\
\delta \mathbf{x}^{+}=\mathbf{T} \delta \mathbf{x}, & \text { if } t \in\left\{t_{s}\right\}, \\
\delta \mathbf{x}^{+}=\delta \mathbf{x}+\sigma v \mathbf{U} \delta \mathbf{x}_{\tau}, & \text { if } t-\tau \in\left\{t_{s}\right\},
\end{array}
$$

where $\delta \mathbf{x}$ represents the $d$-dimensional perturbation from the synchronization manifold 
in the eigensystem of G. $v$ can be considered as a generalized eigenvalue of $\mathbf{G}$. As in the case of the MSF for zero-lag synchrony (see Sec. 3.2) and the one for group synchrony (see Sec. 5.1), we hereby separated the perturbations in the longitudinal direction $(v=1$ or $v=0$ in the case that the row sum of $\mathbf{G}$ is one or zero, respectively) from those in the transverse directions ( $v \neq 1$ or $v \neq 0$, respectively). From Eq. (5.60) the largest Lyapunov exponent $\Lambda(v)$ can be calculated as a function of $v$.

\section{Cluster and group synchrony}

As in the case of smooth systems the MSE as given by Eq. (5.60) can be generalized to group states. Here, we do consider the case of two groups. This case is of particular interest in the study of neural systems since it allows to distinguish between excitatory and inhibitory neurons which behave distinctly different [Izhikevich, 2003, 2004]. The generalization to more than two groups, for example in order to take several types of neurons into account, is straightforward.

In the following, quantities which are associated with the excitatory group or couple into this group are marked by an upper index ${ }^{\mathscr{E}}$, while the quantities of the inhibitory group and the coupling to this have as an upper index ${ }^{\mathscr{I}}$. We consider an excitatory group of $N_{\mathscr{E}}$ nodes and an inhibitory group of $N_{\mathscr{I}}$ elements. The coupling from the excitatory group to the inhibitory group is given by the $N_{\mathscr{I}} \times N_{\mathscr{E}}$ matrix $\mathbf{A}^{(\mathscr{\mathscr { I }})}$, while the $N_{\mathscr{E}} \times N_{\mathscr{I}}$ matrix $\mathbf{A}^{(\mathscr{E})}$ transmits the coupling from the inhibitory group to the excitatory one. The connection between the nodes of the same group are given by the $N_{\mathscr{E}} \times N_{\mathscr{E}}$ matrix $\mathbf{B}^{(\mathscr{E})}$ and the $N_{\mathscr{I}} \times N_{\mathscr{I}}$ matrix $\mathbf{B}^{(\mathscr{I})}$, respectively.

The dynamics of the $i$ th node in the $k$ th group is then given by

$$
\begin{aligned}
\dot{\mathbf{x}}_{i}^{(k)} & =\mathbf{f}^{(k)}\left(\mathbf{x}_{i}^{(k)}\right)+\sigma_{A}^{(k)}{ }_{j=0}^{N_{l}-1} A_{i j}^{(k)} \mathbf{H}^{(k)} \mathbf{x}_{j, \tau}^{(l)}+\sigma_{B}^{(k)}{ }_{j=0}^{N_{k}-1} B_{i j}^{(k)} \mathbf{H}^{(k)} \mathbf{x}_{j, \tau}^{(k)}, & & \text { if } \quad \varphi^{(k)}\left(\mathbf{x}_{i}^{(k)}\right) \neq 0, \\
\mathbf{x}_{i}^{(k),+}= & \mathbf{g}^{(k)}\left(\mathbf{x}_{i}^{(k)}\right), & & \text { if } \quad \varphi^{(k)}\left(\mathbf{x}_{i}^{(k)}\right)=0,
\end{aligned}
$$

where $i=0, \ldots, N_{k}-1, k, l \in\{\mathscr{E}, \mathscr{I}\}$ and $k \neq l$. The coupling strengths of the intra- and inter-group connections are given by the factors $\sigma_{A}^{k}$, and $\sigma_{B}^{k}$, respectively. For national convenience, the propagation delay $\tau$ to be the same for all couplings. Figure 5.8(a) shows a schema of the coupling.

In a group synchrony state the nodes in one group follow the same trajectory $\mathbf{x}_{s}^{(k)} \equiv \mathbf{x}_{i}^{(k)}$, $i=0, \ldots, N_{k}-1$. However, the dynamics between different groups may differ, see also 
Sec. 5.1. The group synchronization manifold is given by

$$
\begin{array}{ll}
\dot{\mathbf{x}}_{s}^{(k)}=\mathbf{f}^{(k)}\left(\mathbf{x}_{s}^{(k)}\right)+\sigma_{A}^{(k)} \mathbf{H}^{(k)} \mathbf{x}_{s, \tau}^{(l)}+\sigma_{B}^{(k)} \mathbf{H}^{(k)} \mathbf{x}_{s, \tau}^{(k)}, & \text { if } \quad \varphi^{(k)}\left(\mathbf{x}_{s}^{(k)}\right) \neq 0, \\
\mathbf{x}_{s}^{(k),+}=\mathbf{g}^{(k)}\left(\mathbf{x}_{s}^{(k)}\right), & \text { if } \quad \varphi^{(k)}\left(\mathbf{x}_{s}^{(k)}\right)=0 .
\end{array}
$$

The variational equations for the perturbations from the synchronous solution, i.e., the MSEs, can be derived to (cf. Eq. (5.60) for the homogenous case):

$$
\dot{\delta \mathbf{x}^{(k)}}=D \mathbf{f}^{(k)}\left(\mathbf{x}_{s}^{(k)}\right) \delta \mathbf{x}^{(k)}+\sigma_{A}^{(k)} v_{A} \mathbf{H}^{(k)} \delta \mathbf{x}_{\tau}^{(l)}+\sigma_{B}^{(k)} v_{B} \mathbf{H}^{(k)} \delta \mathbf{x}_{\tau}^{(k)},
$$

for $t, t-\tau \notin\left\{t_{s}^{(k)}\right\}$ and $t-\tau \notin\left\{t_{s}^{(l)}\right\} . v_{A}$ and $v_{B}$ are the common eigenvalues of the block matrices

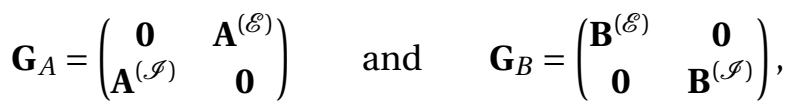

respectively (see Sec. 5.1 and [Sorrentino and Ott, 2007; Dahms, 2011; Dahms et al., 2012]). As before when deriving the MSF for group synchrony of smooth systems, we hereby assumed that $\mathbf{G}_{A}$ and $\mathbf{G}_{B}$ commute such that a common set of eigenvectors exists and the pairs $\left(v_{A}, v_{B}\right)$ of eigenvalues are well defined (see Subsec. 5.1.5 and [Dahms, 2011; Dahms et al., 2012]). $\mathbf{G}_{A}$ and $\mathbf{G}_{B}$ commute if

$$
\begin{aligned}
\mathbf{A}^{(\mathscr{E})} \mathbf{B}^{(\mathscr{I})} & =\mathbf{B}^{(\mathscr{E})} \mathbf{A}^{(\mathscr{E})} \\
\mathbf{A}^{(\mathscr{I})} \mathbf{B}^{(\mathscr{E})} & =\mathbf{B}^{(\mathscr{I})} \mathbf{A}^{(\mathscr{I})}
\end{aligned}
$$

see [Dahms, 2011].

Equation (5.63) has to be complemented by the linearized transition conditions (cf. Eq. (5.60) for the case of zero-lag synchrony):

$$
\begin{array}{rlrl}
\delta \mathbf{x}^{(k),+} & =\mathbf{T}^{(k)} \delta \mathbf{x}^{(k)}, & \text { if } t \in\left\{t_{s}^{(k)}\right\}, \\
\delta \mathbf{x}^{(k),+}=\delta \mathbf{x}^{(k)}+\sigma_{B}^{(k)} v_{B} \mathbf{U}^{(k)} \delta \mathbf{x}_{\tau}^{(k)}, & \text { if } t-\tau \in\left\{t_{s}^{(k)}\right\}, \\
\delta \mathbf{x}^{(l),+}=\delta \mathbf{x}^{(l)}+\sigma_{A}^{(l)} v_{A} \mathbf{U}^{(l)} \delta \mathbf{x}_{\tau}^{(k)} & \text { if } t-\tau \in\left\{t_{s}^{(k)}\right\} .
\end{array}
$$

$\mathbf{T}^{(k)}$ and $\mathbf{U}^{(k)}$ only depend on $\mathbf{x}_{s}^{(k)}$ just before and after the discontinuity and are given 
by

$$
\begin{aligned}
& \mathbf{T}^{(k)} \equiv D \mathbf{g}^{(k)}\left(\mathbf{x}_{s}^{(k)}\right)+\frac{\mathbf{F}^{(k)}\left(\mathbf{x}_{s}^{(k),+}, \mathbf{x}_{s, \tau}^{(k)}, \mathbf{x}_{s, \tau}^{(l)}\right)-D \mathbf{g}^{(k)}\left(\mathbf{x}_{s}^{(k)}\right) \mathbf{F}^{(k)}\left(\mathbf{x}_{s}^{(k)}, \mathbf{x}_{s, \tau}^{(k)}, \mathbf{x}_{s, \tau}^{(l)}\right) D \varphi^{(k)}\left(\mathbf{x}_{s}^{(k)}\right)}{D \varphi^{(k)}\left(\mathbf{x}_{s}^{(k)}\right) \mathbf{F}^{(k)}\left(\mathbf{x}_{s}^{(k)}, \mathbf{x}_{s, \tau}^{(k)}, \mathbf{x}_{s, \tau}^{(l)}\right)} \\
& \mathbf{U}^{(k)} \equiv \frac{\mathbf{F}^{(k)}\left(\mathbf{x}_{s}^{(k)}, \mathbf{x}_{s, \tau}^{(k)}, \mathbf{x}_{s, \tau}^{(l)}\right)-\mathbf{F}^{(k)}\left(\mathbf{x}_{s}^{(k)}, \mathbf{x}_{s, \tau}^{(k)}, \mathbf{x}_{s, \tau}^{(l)}\right) D \varphi^{(k)}\left(\mathbf{x}_{s, \tau}^{(k)}\right)}{D \varphi^{(k)}\left(\mathbf{x}_{s, \tau}^{(k)}\right) \mathbf{F}^{(k)}\left(\mathbf{x}_{s, \tau}^{(k)}, \mathbf{x}_{s, 2 \tau}^{(k)}, \mathbf{x}_{s, 2 \tau}^{(l)}\right)}
\end{aligned}
$$

where we have used the definition

$$
\mathbf{F}^{(k)}\left(\mathbf{x}^{(k)}, \mathbf{x}_{\tau}^{(k)}, \mathbf{x}_{\tau}^{(l)}\right) \equiv \mathbf{f}^{(k)}\left(\mathbf{x}^{(k)}\right)+\sigma_{A}^{(k)} \mathbf{H} \mathbf{x}_{\tau}^{(l)}+\sigma_{B}^{(k)} \mathbf{H} \mathbf{x}_{\tau}^{(k)} .
$$

Note that the transition condition Eqs. (5.66b) and (5.66c) are caused by the delayed coupling between the elements. Equation (5.66b) corresponds to a jump in the same group which occurred a delay time $\tau$ ago. Furthermore, a jump in group $k$ at time $t-\tau$ will also cause a discontinuity in the coupling of the group $l$ at time $t$. This is reflected by Eq. (5.66c).

For details of the derivation see the supplements of [Ladenbauer et al., 2013]. In order to assess the stability of a particular group state, the MSF is then evaluated for the $\left(v_{A}, v_{B}\right)$ pairs. The synchronized state as given by Eq. (5.62) is stable if the largest Lyapunov exponent is negative.

\subsubsection{Synchrony in coupled threshold models}

To demonstrate the potential of the MSF for non-smooth systems, we study in the following a recurrent network of adaptive exponential integrate-and-fire (aEIF) neurons [Brette and Gerstner, 2005]. This neuron model can well reproduce a large variety of subthreshold dynamics and spike patterns observed in cortical neurons [Touboul and Brette, 2008; Naud et al., 2008; Jolivet et al., 2008]. Note that the model employed here uses dimensionless variables. It is a rescaled version of the original model but with fewer parameters; see [Touboul and Brette, 2008]. I will first present the results for zero-lag synchronization in a network of excitatory and inhibitory nodes, where all nodes are modeled by the same dynamics and with the same parameters. Biologically more plausible is it to model the dynamics of the excitatory and inhibitory neurons with different parameters to take into account that their dynamics differ. The MSF for group synchrony allows to do this; the result is presented in the second part of this Subsection. 


\section{Zero-lag synchrony}

The subthreshold dynamics of the $i$ th aEIF neuron follow $(i=0, \ldots, N-1)$ :

$$
\begin{array}{ll}
\dot{V}_{i}=-V_{i}+\exp \left(V_{i}\right)-w_{i}+I+\sigma G_{j=0}^{N-1} G_{i j} s_{j}(t-\tau), & \text { if } \quad V_{i} \neq V_{t h}, \\
\dot{w}_{i}=\frac{a_{w} V_{i}-w_{i}}{\tau_{w}}, & \text { if } V_{i} \neq V_{t h}, \\
\dot{s}_{i}=-\frac{s_{i}}{\tau_{s}}, & \text { if } V_{i} \neq V_{t h} .
\end{array}
$$

If the membrane potential $V_{i}$ reaches the threshold $V_{t h}$, a spike is released and the dynamics is given by

$$
\begin{aligned}
& V_{i}^{(+)}=V_{r}, \\
& \text { if } V_{i}=V_{t h}, \\
& w_{i}^{(+)}=w_{i}^{(-)}+b_{w} \text {, } \\
& \text { if } V_{i}=V_{t h} \text {, } \\
& s_{i}^{(+)}=s_{i}^{(-)}+1, \\
& \text { if } V_{i}=V_{t h} \text {, }
\end{aligned}
$$

where $x_{i}^{+}(t) \equiv \lim _{s \backslash t} x_{i}(s)$ and $x_{i}^{-}(t) \equiv \lim _{s / t} x_{i}(s)$ denote the right-sided and the leftsided limit, respectively.

The terms in Eq. (5.70a) have the following meaning (from left to right): $-V_{i}$ represents a leak current; $\exp \left(V_{i}\right)$ is a fast sodium current at spike initiation; $w_{i}$ is an adaptation current which reflects slowly varying, activity dependent potassium currents; the external driving input is given by $I$; and $s_{i}$ is the strength of an effective synaptic (output) current caused by the $j$ th neuron. The adaptation current $w_{i}$ depends on the membrane potential, where $a_{w} \geq 0$, and $\tau_{w}$ is the adaptation time constant, see Eq. (5.70b). If a neuron is activated, the adaptation current increases, counteracting this activation. Equation (5.70c) describes the dynamics of the synaptic current. The activation of a synapse decays exponentially with relaxation time $\tau_{s}$.

If the membrane potential $V_{i}$ reaches the threshold $V_{t h}$, a spike is triggered and the potential is set to a lower value $V_{r}$; see Eq. (5.70d). The synaptic output current $s_{i}$ is incremented by one which mimics an outgoing spike. The adaptation current $w_{i}$ is increased by the value $b_{W}$ implementing the adaptation mechanism. This spike-based mechanism complements the subthreshold adaption driven by the increasing potential, see Eq. (5.70b). It has been shown that subthreshold adaptation can induce synchrony in pairs of symmetrically coupled excitatory neurons [Ermentrout et al., 2001; Ladenbauer et al., 2012] and that the spike-dependent adaptation can cause networks to split up into 

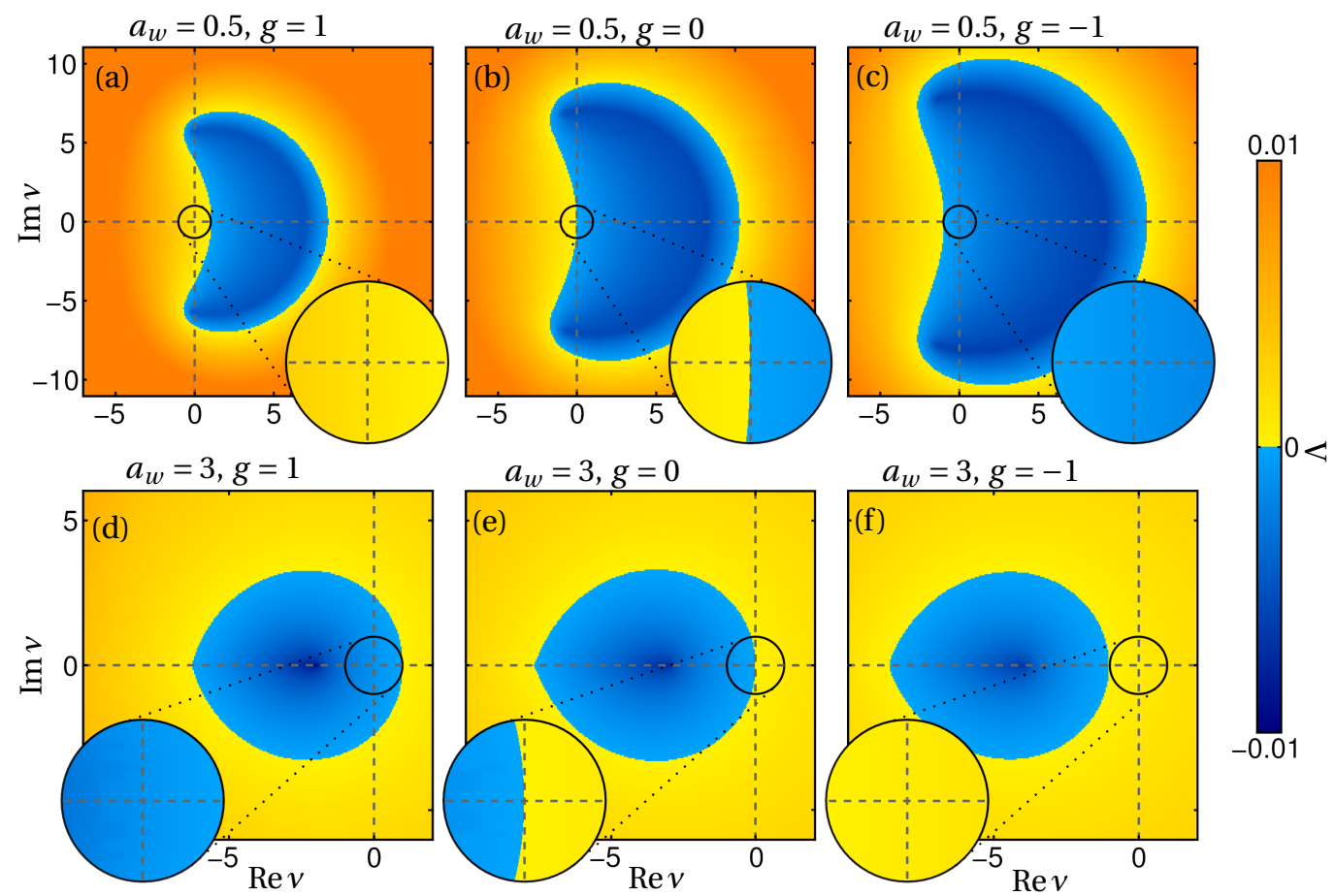

Figure 5.7.: Stability of synchrony for a homogenous population of coupled aEIF neurons as given by Eq. (5.70). The maximum Lyapunov exponent $\Lambda$ (color bar) is plotted as a function of the eigenvalues $v$ of the coupling matrix $\mathbf{G}$. Panels (a), (b) and (c) show the results for strong adaptation $a_{w}$, and panels $(d),(e)$ and $(f)$ depict the results for weak subthreshold adaptation $a_{w}$. The networks are (a) and (d) excitation dominated, (b) and (e) balanced; and (c) and ( $f$ ) inhibition dominated regimes. Parameters: $\lambda=5$, $\tau=0.3, \tau_{w}=10, \tau_{s}=0.3, V_{t h}=5, V_{r}=-5, b_{w}=2.5$. The external input $I$ is chosen such that the oscillation period is always equal to 5 . Circles indicate the unit disc in the eigenvalue plane (insets show blow-up). Figure from [Ladenbauer et al., 2013]. 
phase-locked clusters [Kilpatrick and Ermentrout, 2011]. The framework of the MSF for non-smooth systems allows for studying the two adaptation mechanism for a wide range of networks.

The coupling parameters in Eq. (5.70) are the coupling strength $\sigma$ and the delay time $\tau$. $\mathbf{G}$ is the coupling matrix. The row sum $g$ of $\mathbf{G}$ is assumed to be constant: $g \equiv{ }_{j=0}^{N-1} G_{i j}$, $i=0, \ldots, N-1$; see Sec. 3.1. In contrast to the other parts of this thesis, we do not require that $g$ is equal to one because we want to investigate the case of zero-row sum. Recall that any nonzero row sum can be scaled to one by rescaling of the amplitude of the coupling strength (see Sec 3.1).

Figure 5.7 shows the MSF for the aEIF neurons as given by Eq. (5.70). The parameters are selected such that the dynamics of the aEIF model closely matches the regular spiking dynamics of cortical neurons [Naud et al., 2008; Destexhe, 2009]. Depending on the amount of inhibition three different cases can be distinguished: In panels (a) and (d) networks with dominantly excitatory coupling, i.e., $g=1$, are depicted. The MSF for networks with dominantly inhibitory coupling, i.e., $g=-1$, is shown in panels (c) and (f). Panels (b) and (e) correspond to a situation with a balance of excitation and inhibition, i.e., $g=0$. The subthreshold adaptation $a_{w}$ is weak in panels (a), (b), and (c), while the case of strong adaptation is depicted in panels (d), (e), and (f). The black circle in each panel marks the unit circle. Recall that the eigenvalues of matrices where all entries are positive or zero, or matrices where all entries are negative or zero will always be located inside this circle if $|g|=1$ holds. This follows from Gershgorin's circle theorem [Gerschgorin, 1931; Earl and Strogatz, 2003]. Thus, the MSF inside this circle is of particular interest and is, thus, highlighted in the insets.

Stable synchronization for networks with eigenvalues lying inside the unit circle is predicted for excitation-dominated networks of neurons with strong subthreshold adaptation (panel (d)) and for inhibition dominated networks of neurons with weak subthreshold adaptation (panel (f)). For the networks with a balance of excitation and inhibition stabilizing synchrony by increasing or decreasing the adaptation is infeasible as there are negative and positive values of the largest Lyapunov exponent inside the unit circle for weak as well as strong adaption. In consequence, most realistic networks will not exhibit stable synchrony. These results show that neural adaptation is a key factor in the control of synchrony. 
(a)

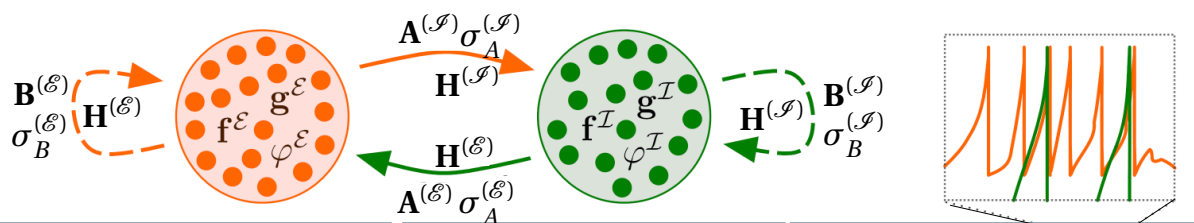

(b)
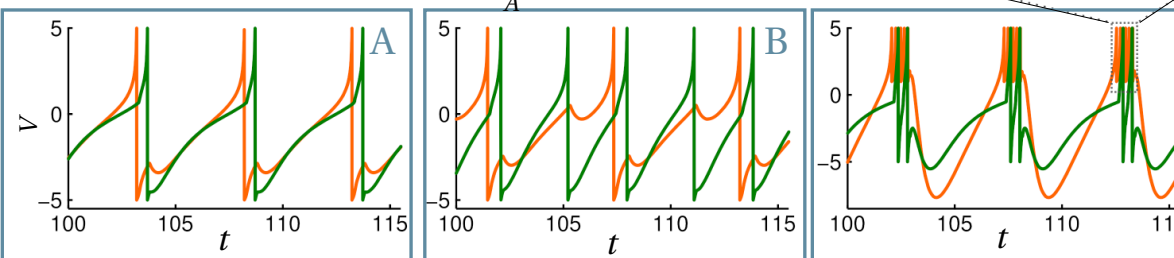

(c)
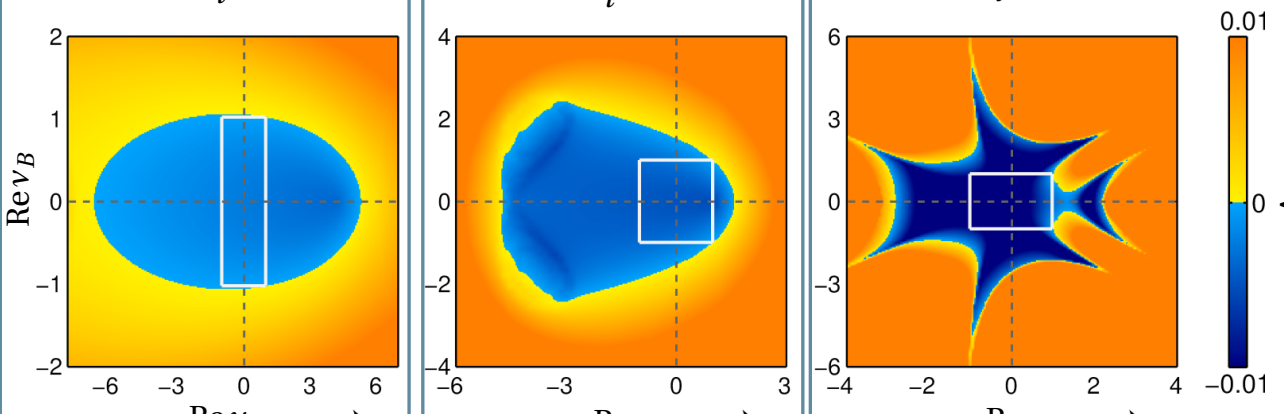

(d)
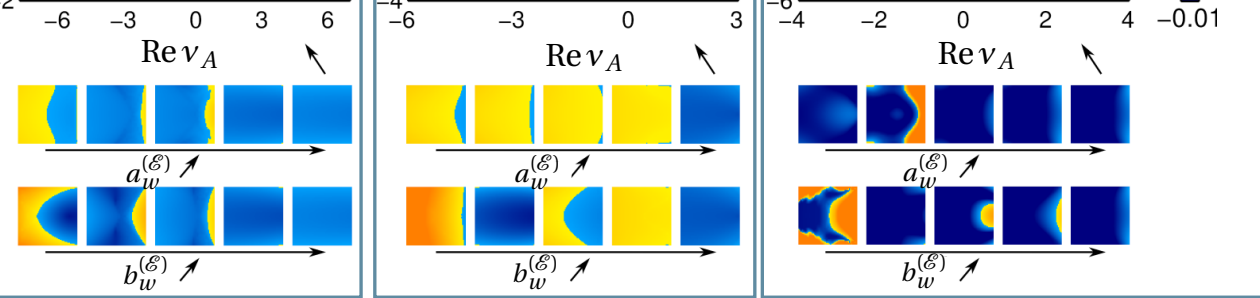

Figure 5.8.: Stability of group synchrony in a network of excitatory $(\mathscr{E})$ and inhibitory (II) neurons which differ in their local dynamics. (a) Scheme of the network according to Eq. (5.71). Solid green and dashed orange arrows correspond to excitatory (positive sign) and inhibitory coupling (negative sign), respectively. (b) Evolution of the membrane voltage $V$ for three different dynamical scenarios $A, B$, and $C$ in the left, middle, and right column, respectively. Orange and green lines mark the voltage $V$ of synchronized excitatory and inhibitory neurons, respectively. The parameter sets for the scenarios A, B, and $C$ are chosen according to Table 5.1. (c) Master stability function (MSF) for each of the three scenarios in (b). The maximum Lyapunov exponent is shown as a function of the real parts of the eigenvalues $v_{A}^{(k)}$ and $v_{B}^{(k)}$. White rectangles indicate the unit square in the eigenvalue plane. (d) Master stability function (MSF) inside the unit square as in (c), but with $a_{w}^{(\mathscr{E})}=0.1,0.2,0.3,0.4,0.5$ (top, left to right) and $b_{w}^{(\mathscr{E})}=0.5,1,1.5,2,2.5$ (bottom, left to right). Figure from [Ladenbauer et al., 2013]. 


\section{Group synchrony}

In the following, an example of group synchrony in a network of aEIF neurons is considered, where one group consist of excitatory nodes and the other one of inhibitory neurons. We choose both groups to be of equal size, i.e., $N_{\mathscr{E}}=N_{\mathscr{I}} \equiv N$. The dynamics of the $i$ th aEIF neuron in $k$ th group is given by $(i=0, \ldots, N-1 ; k, l \in\{\mathscr{E}, \mathscr{I}\})$ :

$$
\begin{aligned}
\dot{V}_{i}^{(k)}= & -V_{i}^{(k)}+\exp \left(V_{i}^{(k)}\right)-w_{i}^{(k)}+I^{(k)} \\
& +\sigma_{A}^{(k)} \quad{ }_{j=0}^{N-1} A_{i j}^{(k)} s_{j}^{(l)}(t-\tau)+\sigma_{B}^{(k)} \quad{ }_{j=0}^{N-1} B_{i j}^{(k)} s_{j}^{(k)}(t-\tau), \\
\dot{w}_{i}^{(k)}= & \frac{a_{w}^{(k)} V_{i}^{(k)}-w_{i}^{(k)}}{\tau_{w}^{(k)}}, \\
\dot{s}_{i}^{(k)}= & -\frac{s_{i}^{(k)}}{\tau_{s}^{(k)}}, \\
V_{i}^{(k,+)}= & V_{r}^{(k)}, \\
w_{i}^{(k,+)=} & w_{i}^{(k,-)}+b_{w}^{(k)}, \quad \text { if } \quad V_{i}^{(k)}=V_{t h}^{(k)}, \\
s_{i}^{(k,+)}= & s_{i}^{(k,-)}+1,
\end{aligned}
$$

where Eq. (5.71a) describes the subthreshold dynamics. Recall that $V_{i}^{(k)}$ is the membrane voltage, $w_{i}^{(k)}$ the adaptation current, $I^{(k)}$ the external driving current and $s_{i}^{(k)}$ the strength of an effective synaptic output current. The strength of the adaptation and the adaptation time constant are given by $a_{w}^{(k)} \geq 0$ and $\tau_{w}^{(k)}$, respectively. $\tau_{s}^{(k)}$ sets the relaxation time of the synapses. Equation (5.71a) describes the spiking event, where $V_{r}^{(k)}$ is the resting potential and $b_{w}^{(k)}$ the increase of the adaptation in the event of a spike. The synaptic output strength is incremented by one. For details of the model see Eq. (5.70) and its description.

We now apply our MSF formalism to the network described in Eq. (5.71), where the excitatory and the inhibitory subpopulations now have different local dynamics (see Fig. 5.8(a)) which is reflected by different parameters in Eq. (5.71). The inhibitory neurons are modeled such that they mimic the dynamics of the fast spiking inhibitory interneurons in the neocortex [Destexhe, 2009]. Their adaptation is weak, while the adaptation of the excitatory population is strong. 
We employ three different parameter sets yielding three different dynamical scenarios to which we refer in the following as scenarios A, B, and C. The corresponding parameters are given in Table 5.1. In Figs. 5.8(b) and (c) in the left and center column scenarios A and B are shown, respectively, where the parameters are chosen such that they mimic the dynamics of the pyramidal neurons in the neocortex [Destexhe, 2009]. Figures 5.8(b) and (c) depict in the right column the scenario $\mathrm{C}$ where intrinsically bursting neurons are modelled, which, when activated, produce repeated events of rapid spiking. The bursting is caused by an increased reset membrane potential $V_{r}^{(\mathscr{E})}$ [Naud et al., 2008].

The MSFs for the three different spiking behaviours A, B, and C are shown in Fig. 5.8(c) as a function of the real parts of the eigenvalue $v_{A}$ and $v_{B}$, where the imaginary part was set to zero, i.e., $\operatorname{Im} v_{A}=\operatorname{Im} v_{B}=0$. The unit square is marked by a white rectangle. Recall that the eigenvalues of symmetric matrices with unity row sum and all entries being non-negative are always contained in the unit square. This follows from Gershgorin's circle theorem [Gerschgorin, 1931; Earl and Strogatz, 2003]. For the matrices employed here this applies: We chose positive entries for the matrices and modeled the excitatory and the inhibitory characteristic of the nodes by positive $\left(\sigma_{A}^{\mathscr{I}}, \sigma_{B}^{\mathscr{E}}>0\right)$ and negative $\left(\sigma_{A}^{\mathscr{E}}, \sigma_{B}^{\mathscr{S}}<0\right)$ coupling strengths, respectively. For all three dynamical scenarios we find that the maximum Lyapunov exponent $\Lambda$ is negative inside the unit square meaning that group synchrony is stable for the employed parameters sets and all networks fulfilling the commuting criteria (see Eq. (5.65)).

Figure 5.8(d) shows the effects of increasing subthreshold adaption, i.e, increasing $a_{w}^{(\mathscr{E})}$ (upper row), and increasing spike-based adaption, i.e, increasing $b_{w}^{(\mathscr{E})}$ (lower row), on the MSF in the unit square. Clearly visible is the strong effect of the adaptation on the stability of synchronization: For weak adaptation the Lyapunov exponent becomes positive in parts or for the whole unit square, indicating the loss of stability.

\subsection{CONCLUSION}

Besides zero-lag synchrony, cluster and group synchrony are dynamical states which can be observed in a variety of biological systems ranging from central pattern generation and animal locomotion to population dynamics. While cluster synchrony refers to a state where the network splits into several clusters which undergo the same dynamics but with a constant phase shift, group synchrony describes a state where the nodes of one group are in zero-lag synchrony but the different groups can have different dynamics. In this Chapter, I have discussed the stability problem of these states and have showed that the 
master stability function (MSF) can be generalized to treat these states if the topology fulfills a certain restriction. In fact, it is necessary that the topology on the level of groups is a unidirectional ring meaning that each group couples only to one other group. The MSF for group synchrony as well as matrices fulfilling the necessary restriction are characterized by a discrete rotational symmetry in the eigenvalue plane. This symmetry is demonstrated on the example of the saddle-node infinite period (SNIPER) bifurcation model which is generic for type-I excitability as discussed in Chapter 4 .

The above restriction on the topology can be lifted in the case of commuting coupling matrices. In the case of two groups, this allows for taking intergroup and intragroup coupling with different delay times into account. The MSF translates this network to a motif of two coupled nodes with self-feedback, where the coupling and the self-feedback are characterized by potentially different delays. Panchuk et al. [2013] showed that such a motif of two coupled FitzHugh Nagumo systems can exhibit zero-lag synchrony as well as antisynchronization and bursting. We have employed the MSF for group synchrony to these different dynamics which yields their stability in large, complex networks. It emerges that purely excitatorily coupled networks can exhibit stable synchrony in all three dynamical scenarios while in networks with excitatory and inhibitory coupling the stability depends on the type of dynamics.

The MSF - for zero-lag and for group synchrony - can be extended to treat non-smooth systems which is of particular interest for applications in neurosciences where integrate and fire models (IF) play an important role. In these models, the discontinuity arises from the fact that spikes are not explicitly modeled but that the voltage is set back to a rest state after it reaches a threshold. In the MSF the discontinuity can be overcome with the help of transition matrices. We have first demonstrated the method on the example of zero-lag synchrony in a network of adaptive exponential integrate-and-fire (aEIF) neurons where it has been shown that subthreshold adaptation is a crucial parameter in the control of synchrony. Furthermore, the method has been applied to group synchrony in a network of aEIF neurons where one group consisted of excitatory neurons and the other one of inhibitory ones. Depending on the parameters three distinctly different dynamical states including bursting can be observed. For all scenarios it shows that a decreasing adaptation strength leads to destabilization of synchrony. 


\begin{tabular}{|c|c|c|c|c|}
\hline & \multirow[b]{2}{*}{ Parameters } & \multicolumn{3}{|c|}{ Dynamical scenario } \\
\hline & & A & B & $\mathrm{C}$ \\
\hline & $\tau$ & 0.1 & 0.1 & 0.1 \\
\hline \multirow{10}{*}{ 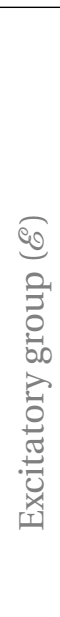 } & $V_{r}^{(\mathscr{E})}$ & -5 & -5 & 2 \\
\hline & $I^{(\mathscr{E})}$ & 3.725 & 3.725 & 25 \\
\hline & $\sigma_{A}^{(\mathscr{E})}$ & -5 & -5 & -25 \\
\hline & $\sigma_{B}^{(\mathscr{E})}$ & 5 & 5 & 25 \\
\hline & $a_{w}^{(\mathscr{E})}$ & 0.5 & 0.5 & 0.5 \\
\hline & $b_{w}^{(\mathscr{E})}$ & 2.5 & 2.5 & 2.5 \\
\hline & $\tau_{w}^{(\mathscr{E})}$ & 10 & 10 & 10 \\
\hline & $\tau_{s}^{(\mathscr{E})}$ & 0.2 & 0.2 & 0.2 \\
\hline & $\tau_{t h}^{(\mathscr{E})}$ & 5 & 5 & 5 \\
\hline & $V_{r}^{(\mathscr{E})}$ & -5 & -5 & -5 \\
\hline \multirow{8}{*}{ 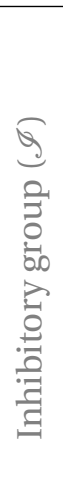 } & $I^{(\mathscr{I})}$ & 0 & 1.5 & 0 \\
\hline & $\sigma_{A}^{(\mathscr{I})}$ & 5 & 5 & 25 \\
\hline & $\sigma_{B}^{(\mathscr{I})}$ & -5 & -5 & -25 \\
\hline & $a_{w}^{(\mathscr{J})}$ & 0.1 & 0.1 & 0.1 \\
\hline & $b_{w}^{(\mathscr{I})}$ & 0.25 & 0.25 & 0.25 \\
\hline & $\tau_{w}^{(\mathscr{I})}$ & 10 & 10 & 10 \\
\hline & $\tau_{s}^{(\mathscr{I})}$ & 0.5 & 0.5 & 0.5 \\
\hline & $\tau_{t h}^{(\mathscr{I})}$ & 5 & 5 & 5 \\
\hline
\end{tabular}

Table 5.1.: Parameters used for Figs. 5.8(b) and (c). 


\section{6 | ZERO-LAG AND CLUSTER SYNCHRONY: TOWARDS APPLICATIONS}

Chapters 4 and 5 studied zero-lag, cluster and group synchrony with the help of the master stability function (MSF). Prerequisite for applying the MSF for zero-lag synchronization is that all nodes are identical and that the coupling delays are equal; see Sec. 3.1. For group synchrony, it is required that the nodes in one group are alike and that the delays for links coupling into the same group are the same; see Sec. 5.1. Depending on the topology a reduction of the delays occurring in a network is possible but only in rare cases this will reduce the number of delays to one [Lücken et al., 2013]. Two ore more discrete delays in the coupling to one group can only be considered with the MSF in the framework of commuting matrices; see Subsec. 5.1.5. However, real-world networks are not limited to cases well described by commuting coupling matrices and discrete delay times but are often characterized by a complex topology, for example of random or small-world type, and heterogeneous delays. Furthermore, heterogeneities in the local dynamics of the nodes will always be present in applications.

The aim of this Chapter is to study the application of the theory developed in the previous Chapters to networks characterized by heterogeneities. We investigated the interplay between delay heterogeneities and topology in [Cakan et al., 2014]. The results are discussed and presented in Sec. 6.1. Section 6.2 investigates cluster states in chemical oscillators which are slightly non-identical. Depending on the applied voltage smooth or non-smooth oscillations can be observed where the latter made it necessary to extent the existing theory. Section 6.2 is based on [Blaha et al., 2013]. 


\subsection{HETEROGENOUS DELAYS IN COMPLEX NETWORKS}

This Section investigates synchronization and other space-time patterns in the presence of heterogeneous delay times in neural networks. It closely follows [Cakan et al., 2014].

In neural networks the propagation speed and, thus, the time delay between neurons can vary between 1 and $100 \mathrm{~mm} / \mathrm{ms}$ where the nerve conduction velocity is determined by the length, the diameter, and the kind of the axons between the neurons [Koch, 1999]. It is, therefore, of particular interest how robust the results on synchronization in neural networks obtained with the MSF and discussed in Chapter 4 are towards delay heterogeneities. For heterogeneities in the nodes it is well known that they hinder or prevent synchronization [Strogatz, 2000; Sun et al., 2009]. Here, we will study whether this is also the case if the heterogeneity affects the delay. In particular, we consider two discrete delay times as well as unimodal and bimodal delay distributions. In the context of the brain, a bimodal distribution is a good first approximation if the coupling on two different length scales is considered, i.e., nearby connections within brain areas associated with short delays, and links between distant areas characterized by long delays.

\subsubsection{Model}

As in Chapter 4, we consider networks of FitzHugh-Nagumo systems which are generic for type-II excitability; see Sec. 4.1.4 and [FitzHugh, 1961; Nagumo et al., 1962; Lindner et al., 2004]. A network of $N$ coupled FitzHugh-Nagumo systems with heterogeneous delays is given by

$$
\begin{aligned}
\varepsilon \dot{u}_{i} & =u_{i}-\frac{u_{i}^{3}}{3}-v_{i}+K{ }_{j=0}^{N-1} G_{i j} u_{j}\left(t-\tau_{i j}\right)-u_{i}(t), \\
\dot{v}_{i} & =u_{i}+a,
\end{aligned}
$$

where $u_{i}$ and $v_{i}$ denote the activator and inhibitor variable of the nodes $i=0, \ldots, N-1$, respectively, and $\varepsilon$ is a time-scale separation parameter and typically small (here we will use $\varepsilon=0.01$ ), meaning that $u_{i}$ becomes a fast variable, while $v_{i}$ changes slowly. Recall that in the uncoupled system ( $K=0), a$ is the threshold parameter: Due to a supercritical Hopf bifurcation the systems is excitable for $a>1$, while for $a<1$ it exhibits selfsustained periodic firing. For details on the model see Sec. 4.1.4. Here, we choose $a=1.3$ such that the system operates in the excitable regime. 
$\mathbf{G}=\left\{G_{i j}\right\}$ is the coupling matrix. An invariant synchronization manifold will only exist if $\mathbf{G}$ has a constant row sum; without loss of generality we assume the row sum to be unity, i.e., $\quad{ }_{j} G_{i j}=1$, for all $i=0, \ldots, N-1$; see Subsec. 3.1. We construct the matrix $\mathbf{G}$ by setting the entry $G_{i j}$ equal to one if the $j$ th node couples into the $i$ th node, otherwise the entry is set to zero. In the following, we will construct regular, small-world, and random networks in this way; see Sec. 2.3. After repeating this procedure for all entries of $G_{i j}$, we normalize each row to unity. The overall real-valued coupling strength is given by $K$. Throughout this Section, we use bidirectional coupling, which means that signals can always be transmitted in both directions. This makes $\mathbf{G}$ a symmetric matrix (before we normalize each row sum to unity). $\mathbf{T}=\left\{\tau_{i j}\right\}$ is the delay matrix, i.e., $\tau_{i j}$ is the time the signal needs to propagate from the $j$ th node to the $i$ th node.

The fixed point of system (6.1) is given by

$$
\begin{aligned}
& u_{i} \equiv u_{*}=-a, \\
& v_{i} \equiv v_{*}=u^{*}-\frac{u_{*}^{3}}{3}, \quad i=0, \ldots, N-1 .
\end{aligned}
$$

As discussed in Sec. 4.1.4, the FitzHugh-Nagumo system undergoes a large excursion in the phase space if perturbed sufficiently strong from the fixed point. This excursion mimics the spike of a neuron. The coherence of spiking - of a single node or of nodes coupled in a network - can be measured by considering the inter-spike intervals (ISIs), i.e., the time between two successive spikes [Hövel, 2010].

\subsubsection{Unimodal delay distributions in complex networks}

The first step in going from one discrete delay time to more realistic models with heterogeneous delay times is to consider a unimodal distribution. In the following, we choose the elements of the delay matrix $\mathbf{T}$ randomly from a normal distribution $\mathscr{N}\left(\tau_{\mu}, \sigma_{\tau}^{2}\right)$ with mean $\tau_{\mu}$ and standard deviation $\sigma_{\tau}$.

In this sense, in Chapter 4, system (6.1) had a $\delta$-distribution of the delay times. For excitatory coupling, i.e., all entries of $\mathbf{G}$ are positive, it was shown that synchronized spiking with an inter-spike interval (ISI) of $\tau_{\mu}$ is always stable independently of coupling strength and delay time as long as both are large enough to induce any spiking at all. In this Section, we will discuss how robust these results are if we increase $\sigma_{\tau}$. In particular, we will focus on the effect of the underlying topology - regular, small-world, or random - on the dynamics. These topologies are constructed as follows: In a regular ring network each node is connected with equal strength to its $k$ nearest neighbors to the left and to the 
right, i.e., the node degree is $2 k$. If additional excitatory links are added with a probability $p$ to such a regular network, a small-world network arises [Watts and Strogatz, 1998; Monasson, 1999; Newman and Watts, 1999]. In a random network each node is linked with probability $p$ to every other node [Rapoport, 1957; Solomonoff and Rapoport, 1951; Erdős and Rényi, 1959, 1960]. See Sec. 2.3 for a review of the different network models.

Depending on the distribution width, the topology, and initial conditions there are essentially three different types of dynamics observable: Highly-synchronous spiking, spiking, and global amplitude death, from which the two spiking types can be subdivided into several subclasses.

\section{Spiking patterns and amplitude death}

Highly-synchronous spiking If the distribution width $\sigma_{\tau}$ is sufficiently small, stable synchronization with an ISI of $\tau_{\mu}$ will persist, while the spikes will be broader than for a delta distribution. The quality of synchronization can be measured using the Kuramoto order parameter $R$, see Sec. 3.3. Recall that $R=1$ corresponds to a perfectly synchronized state, while $R \approx 0$ means that a network spikes asynchronously. We consider a network as highly synchronized if $R>0.99$ after all transient effects have vanished.

Spiking For intermediate $\sigma_{\tau}$, different dynamical subclasses can be observed where the network still exhibits spikes, but not necessarily in a highly synchronized manner; we can distinguish approximate synchronization, traveling disruptions, and partial amplitude death.

The first scenario is that all nodes in the network still synchronize but because of the non-zero width of the delay distribution the incoming spikes do not arrive exactly at the same time but with some slight deviations. Thus, $R$ drops below 0.99, i.e., the network is not any longer highly synchronized. Figure 6.1(a) shows as an example the time series of approximate synchronization: The spike times are marked by red dots in panel (a) and the Kuramoto order parameter $R(t)$ is plotted in panel (b). After some transienst time the spikes synchronize but not perfectly. Thus, $R$ remains below 0.99 .

A fairly well synchronized behavior is the most common spiking pattern, but in regularring structures as well as in some small-world realizations a different type of behavior can be observed as well: In Fig. 6.2(a), a disruption travels along many nodes in a ring network without causing amplitude death in the network. Such traveling disruptions 


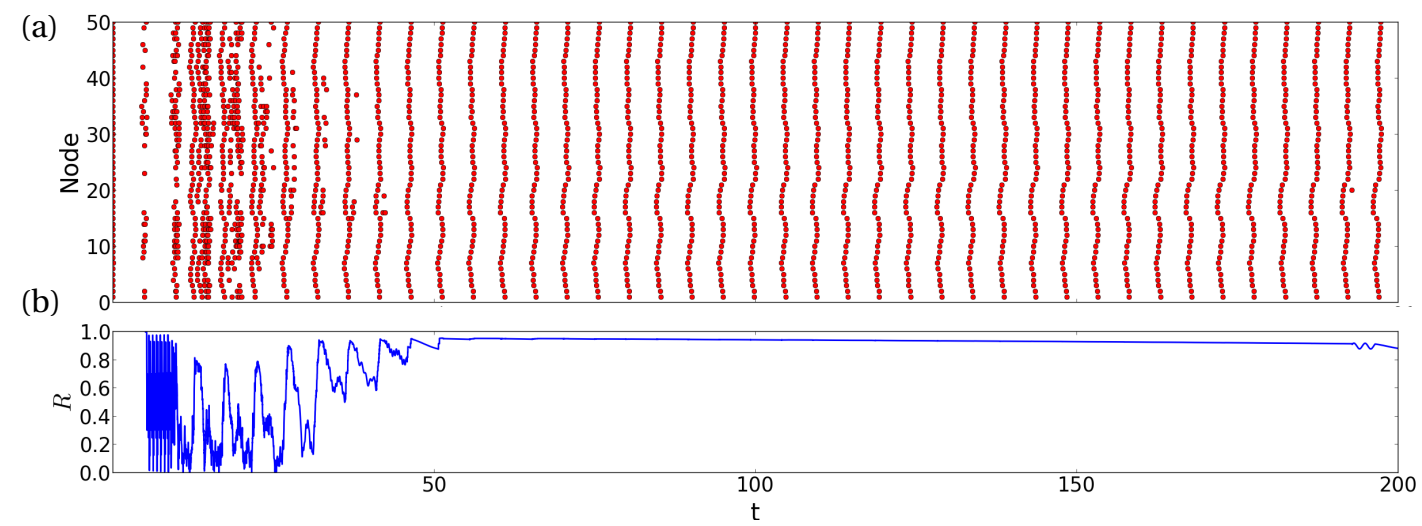

Figure 6.1.: (a) Approximately synchronized spiking pattern (red dots) and (b) order parameter $R$ (blue line) $v$ s. time for a small-world network with a delay distribution with standard deviation $\sigma_{\tau}=0.2$. Parameters: $N=50, p=0.51, k=2, K=1.0$, $a=1.3, \varepsilon=0.01$ and $\tau_{\mu}=5$. The initial condition is $u_{i}=-a, v_{i}=a-a^{3} / 3$ for all $i=0, \ldots, 49$. The history function of all nodes is the spiking state. Figure from [Cakan et al., 2014].

can arise for fairly high standard deviations, e.g., in Fig. 6.2, $\sigma_{\tau}=0.2$. However, the probability for this behavior is quite low since amplitude death is much more common as will be discussed later.

Furthermore, networks can be observed where only a subset of nodes spikes, while the other nodes undergo partial amplitude death. This is the case if, by chance, in a fairly isolated subnetwork the deviation of delay times is smaller than the deviation in the whole network. In large random networks the probability for this is small, since fairly isolated subnetworks arise rarely as they require some kind of ordered structure in the network. In the case of small-world networks though, it is more likely that a subnetwork can maintain stably synchronized spiking while other regions of the network undergo amplitude death. This scenario is depicted in Fig. 6.3. One can also observe cases where a part of the network stops spiking temporarily but then gets excited again and fires synchronously with the rest of the network. This reanimation is also observable in Fig. 6.3 around node no. 43.

Global Amplitude death If $\sigma_{\tau}$ becomes too large, amplitude death is induced, i.e., all nodes remain in the fixed point $\left(u_{*}, v_{*}\right)$, see Eq. (6.2), and no spikes are released. 


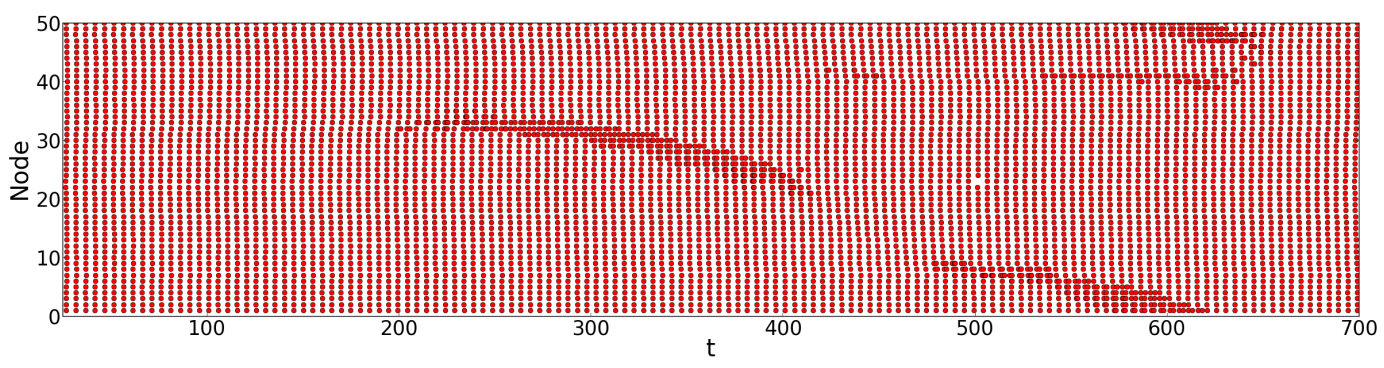

Figure 6.2.: Spiking patterns showing traveling disruptions in a regular ring network with $N=50$ nodes and $\sigma_{\tau}=0.2$. Red dots indicate spikes. Other parameters as in Fig. 6.1. Figure from [Cakan et al., 2014].

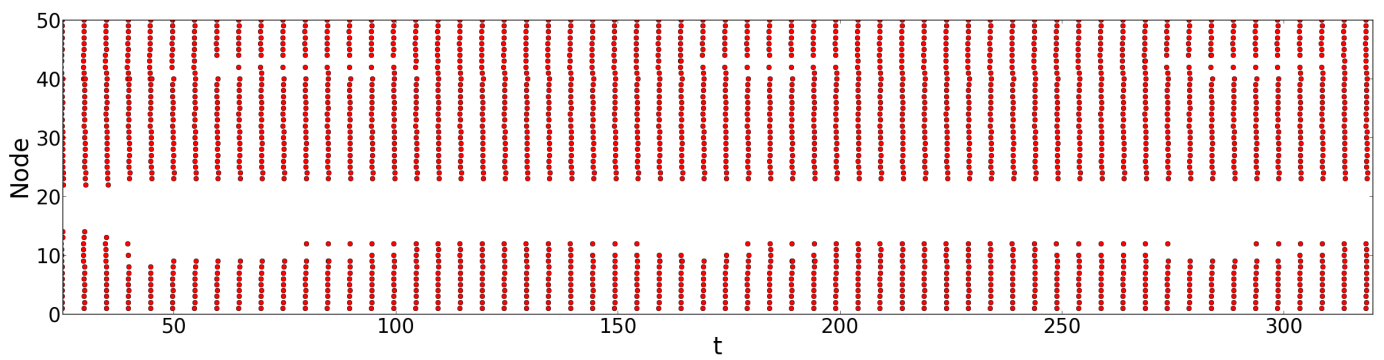

Figure 6.3.: Spiking pattern showing partial amplitude death in a small-world network $(N=50, p=0.51)$ for a delay distribution with standard deviation of $\sigma_{\tau}=0.12$. Red dots mark spikes. Other parameters as in Fig. 6.1. Figure from [Cakan et al., 2014]. 


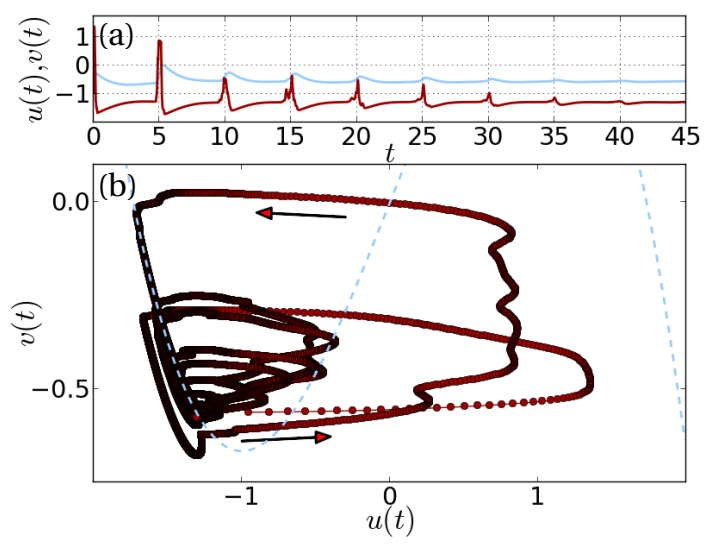

Figure 6.4.: (a) Time series of $u$ (red solid) and $v$ (blue dotted), and (b) phase space of a single node undergoing amplitude death. The node is part of a small-world network ( $N=50, p=0.51$ ) with $\sigma_{\tau}=0.15$. (b) Trajectory (red solid) and $u$-nullcline (blue dashed line). Other parameters as in Fig. 6.1. Figure from [Cakan et al., 2014].

Amplitude death is caused by two different factors. First, recall that the nodes operate in the excitable regime and only spike if perturbed sufficiently strong from the fixed point. This can be due to the coupling or due to external noise. For the intermediate coupling strength of $K=1$ we use, each node needs excitation from many neighbors at the same time to spike. As $\sigma_{\tau}$ increases, the probability that enough spikes from the neighboring neurons arrive sufficiently close in time decreases. Furthermore, the nodes' neighbors which spike too early or too late and which are therefore already or still in the fixed point $\left(u_{*}, v_{*}\right)$ will pull the node back as they give rise to a negative coupling term of the form $u_{*}-u_{i}$ in Eq. (6.1), where we considered the effect on the $i$ th node.

Secondly, even if a spike can be excited by the arriving spikes, for large $\sigma_{\tau}$ retarded spikes are likely to perturb the trajectory of the already spiking node. The result is a spike with a low amplitude, which will be fed back into the network. If this happens on a global scale, i.e., if there is no fairly isolated subnetwork which can maintain the amplitude of the spikes, the spikes will be damped in the course of time and will eventually lead to global amplitude death.

Both of the mentioned effects can be seen in the trajectory depicted in Fig. 6.4. The first effect prevents the trajectory to reach the far right nullcline (dashed blue) in the phase space, which would have been reached in the absence of the negative force pulling it to the left. The second effect causes the trajectory to wiggle around on its round trip, 
instead of allowing a smooth course. This is due to excitations arriving during the round trip which pull the trajectory back and forth. Both effects lead to a decreasing amplitude and eventually to amplitude death.

\section{Statistical analysis}

The type of dynamics taking place on a network depends on the topology, the width of the delay distribution, and on initial conditions. For a systematic study, we calculate the probabilities $p_{s}\left(\sigma_{\tau}\right)$ and $p_{h}\left(\sigma_{\tau}\right)$ that the network shows any kind of spiking behavior or highly synchronized spiking, respectively, for a given realization of the delay matrix $\mathbf{T}$ with the standard deviation $\sigma_{\tau}$ and a given realization of the network topology. Note that $p_{h} \leq p_{s}$ as the highly synchronized networks are a subset of the spiking ones. Figure 6.5 shows $p_{h}\left(\sigma_{\tau}\right)$ (dotted lines) and $p_{s}\left(\sigma_{\tau}\right)$ (solid lines) for (a) a regular ring, (b) a smallworld network, and (c) a random network, for different network sizes of $N=20$ (blue line), $N=50$ (red line), and $N=100$ (green line) nodes.

Figure 6.5 reveals that for each type of topology, a threshold value $\bar{\sigma}_{\tau}$ exists above which global amplitude death almost certainly sets in, i.e., $p_{s}\left(\sigma_{\tau}\right) \approx 0$ for $\sigma_{\tau}>\bar{\sigma}_{\tau}$ (see solid lines in Fig. 6.5). Comparing the different topologies, it is interesting to note that $\bar{\sigma}_{\tau}$ is smaller, about 0.15 for $N=100$, in the case of the small-world and the random network as compared to the regular network, where $\bar{\sigma}_{\tau}$ is about 0.2. Furthermore, in small-world and random networks $\bar{\sigma}_{\tau}$ is preceded by a steep sigmoidal transition, while in the case of the regular network the transition is less steep and characterized by a long tail making it difficult to clearly define $\bar{\sigma}_{\tau}$. As discussed later in detail, the reason for this behavior is that in regular networks often spiking subnetworks survive, which is very unlikely for small-world and random networks. Noteworthy is also that in the case of regular networks global amplitude death occurs later for larger networks, because it is more likely that spiking subnetworks exist in a large network compared to a small one. In contrast, in small-world and random networks small networks survive longer.

The topology of the network is even more critical when considering the fraction of highly synchronized networks, i.e., the curves for $p_{h}$ (dashed lines in Fig. 6.5). A particularly interesting phenomenon is the non-monotonic behavior of $p_{h}$ in the regular ring networks (Fig. 6.5(a) dashed). While the fraction of highly synchronized networks rapidly drops for small $\sigma_{\tau}$, it rises again as the fraction of spiking networks falls. For large $\sigma_{\tau}, p_{h}$ and $p_{s}$ converge, i.e., only highly synchronized networks survive for higher $\sigma_{\tau}$. This is certainly not the case for intermediate $\sigma_{\tau}$, where most of the surviving networks are not highly-synchronized. This counterintuitive behavior of $p_{h}$ can be explained by the fact that for intermediate $\sigma_{\tau}$ the network splits into subnetworks as shown in Fig. 6.6: Panel 


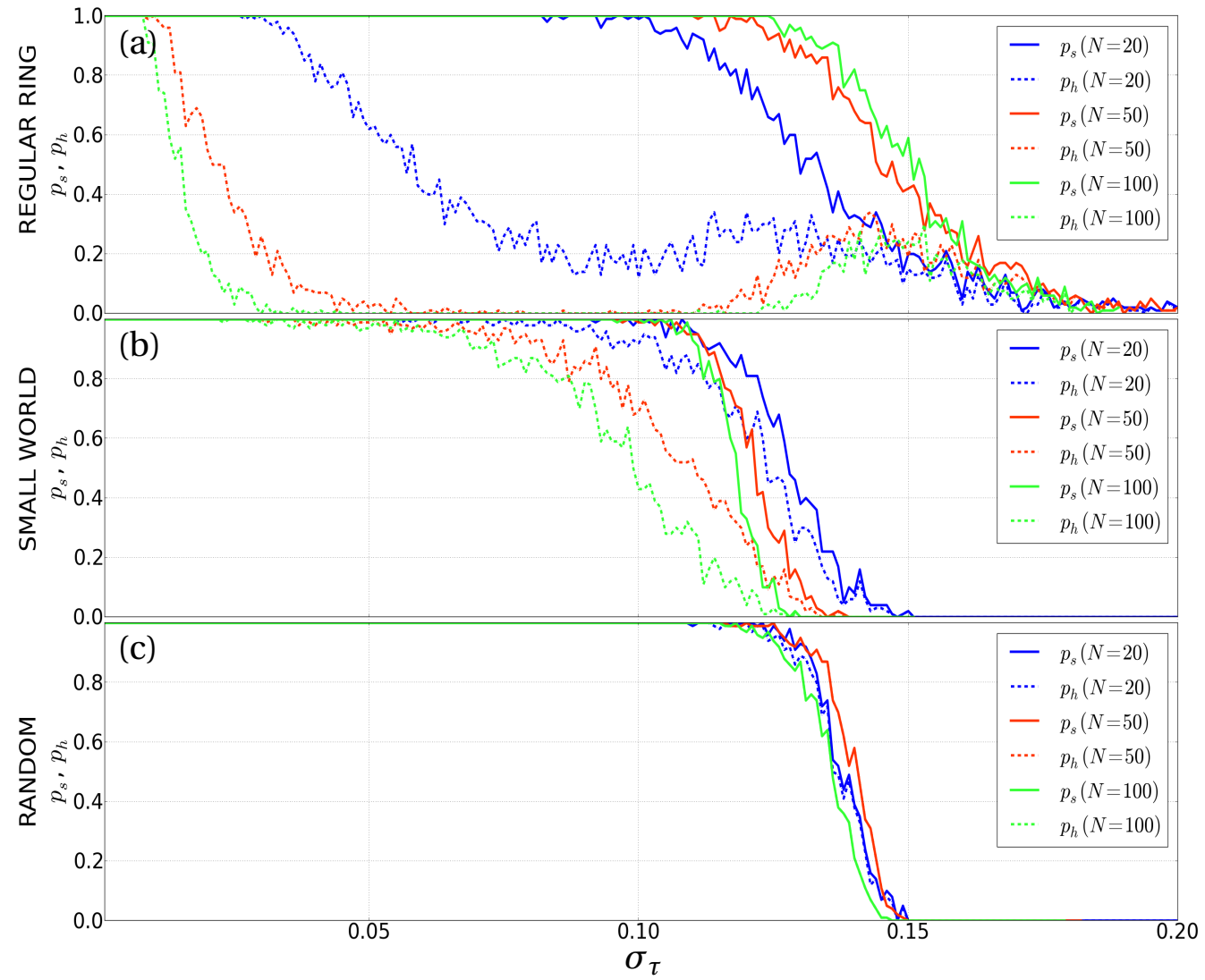

Figure 6.5.: Probability of spiking $p_{s}$ (solid lines) and of highly synchronized spiking $p_{h}$ (dotted lines) vs. standard deviation of delay distribution $\sigma_{\tau}$ for (a) regular ring networks with $k=2$, i.e, each node is connected to its nearest and next-nearest neigbor to the left and to the right, (b) small-world networks with $k=2$ and $p=0.51$, and (c) random networks with $p=0.51$ and different network sizes $N=20$ (blue), $N=50$ (red), $N=100$ (green). Other parameters as in Fig. 6.1. Figure from [Cakan et al., 2014]. 
(a)

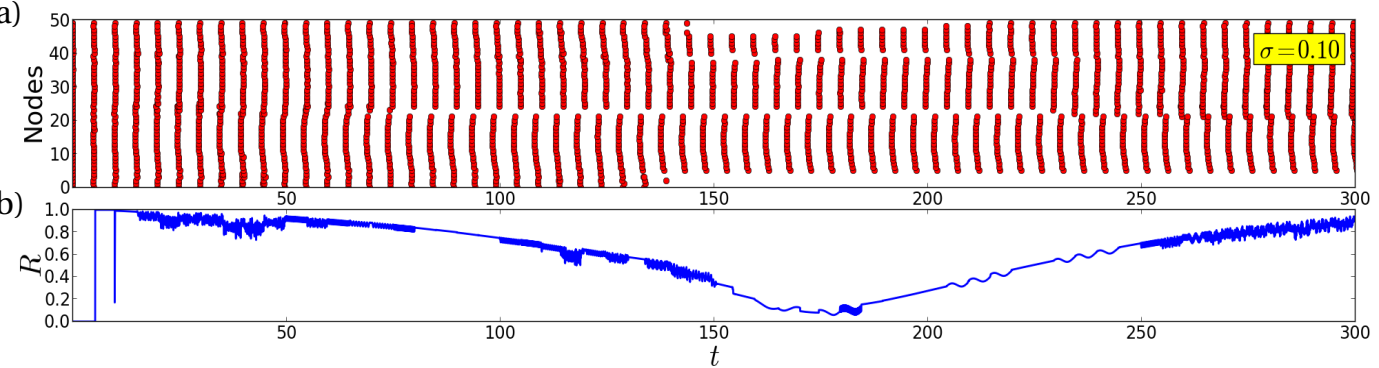

(c)

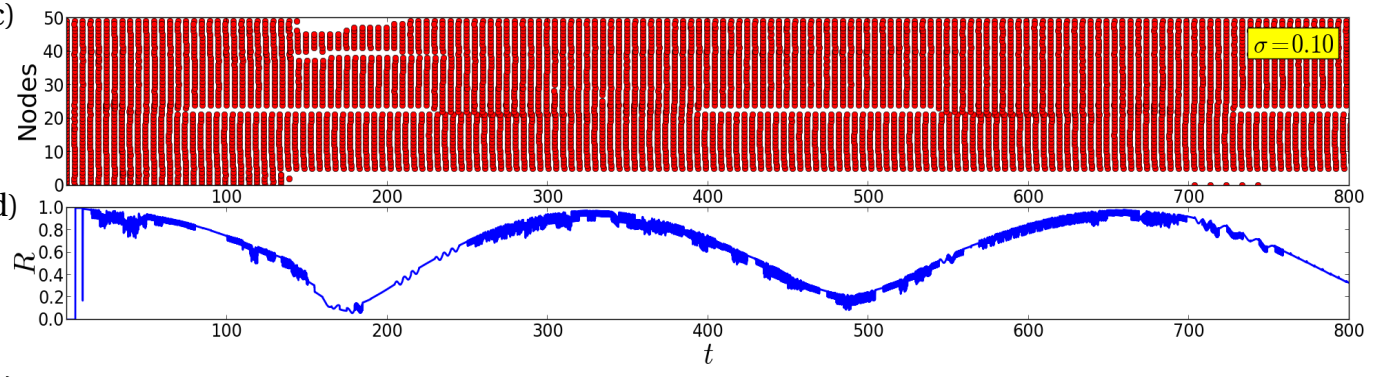

(e)

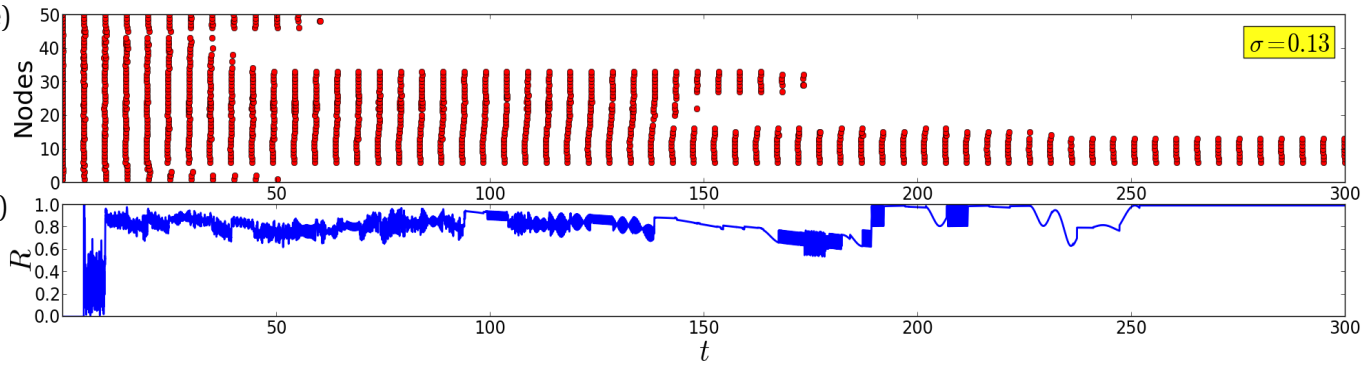

Figure 6.6.: Spiking patterns showing subnetwork synchronization in a regular ring network with $N=50$ nodes for different $\sigma_{\tau}$ : (a)-(d) $\sigma_{\tau}=0.1 ;(e),(f) \sigma_{\tau}=0.13$. Panel (a),(c),(e): Red dots indicate spikes. Panel (b),(d),(f): Kuramoto order parameter $R$. Note that (c),(d) show the same simulation as (a),(b) for a longer time series. Other parameters as in Fig. 6.1. Figure from [Cakan et al., 2014]. 
(a) shows the spiking pattern of a regular ring for intermediate $\sigma_{\tau}=0.1$, i.e., a value for which almost all networks are spiking in a highly asynchronous manner. Panel (b) depicts the corresponding Kuramoto order parameter $R$. Panels (c) and (d) show the same as panels (a) and (b) but for a longer time series up to $t=2000$. It can be clearly seen that after some transient time $(t \approx 500)$, the network consists of two different subnetworks, at times separated by a patch of partial amplitude death. We refer to this state as subnetwork synchronization in the sense that the nodes are synchronized in subnetworks which are not synchronized to each other. Simulations indicate that these subnetworks survive for infinitely long times and do not synchronize to each other. As a consequence the two networks have slightly different ISIs resulting in a beating behavior: For a while the networks are almost synchronized, then their spiking times drift apart leading to slow oscillations in the Kuramoto order parameter. Thus, most of the times $R$ is much smaller than 0.99 and thus the network is not classified as highly synchronized. Because of the slightly different ISIs we do not use the term cluster synchrony here. Recall that we required that for a cluster state the dynamics in the clusters are the same but with a constant phase shift between the clusters; see Chapter 5 .

If $\sigma_{\tau}$ is increased above approximately 0.13, the system either exhibits global amplitude death, or the spiking in only one of the subnetworks dies out. The nodes in the surviving subnetwork remain highly synchronized, since they all interact and the distribution width is not yet too large. Thus, almost all networks which do not undergo global amplitude death are highly synchronized (recall that in the definition of the Kuramoto order parameter given by Eq. (3.19) only spiking nodes are considered). For even larger $\sigma_{\tau}$, global amplitude death can be observed in almost all network realizations.

In contrast, in small-world networks a different behavior can be observed: $p_{s}$ and $p_{h}$ do not coincide for large $\sigma_{\tau}$ but the spiking networks survive longer than the highly synchronized networks; see Fig. 6.5(b). The reason is that in a small-world network, subnetworks do not easily arise because the additional long range links connect the different parts of the network. However, more and more disruptions occur for larger $\sigma_{\tau}$. They decrease $R$ and are thus responsible for the fact that less highly synchronized network persist.

Neither traveling disruptions nor partial amplitude death can be observed in a random network. Therefore, almost all surviving networks are highly synchronized, as shown in Fig. 6.5(c). 


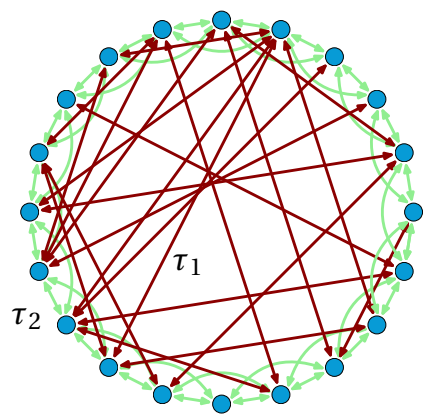

Figure 6.7.: Example of a small-world network, where inner connections (red arrows) have a delay $\tau_{1}$, while the outer connections of the underlying regular ring (green arrows) are characterized by a delay $\tau_{2}$. Figure from [Cakan et al., 2014].

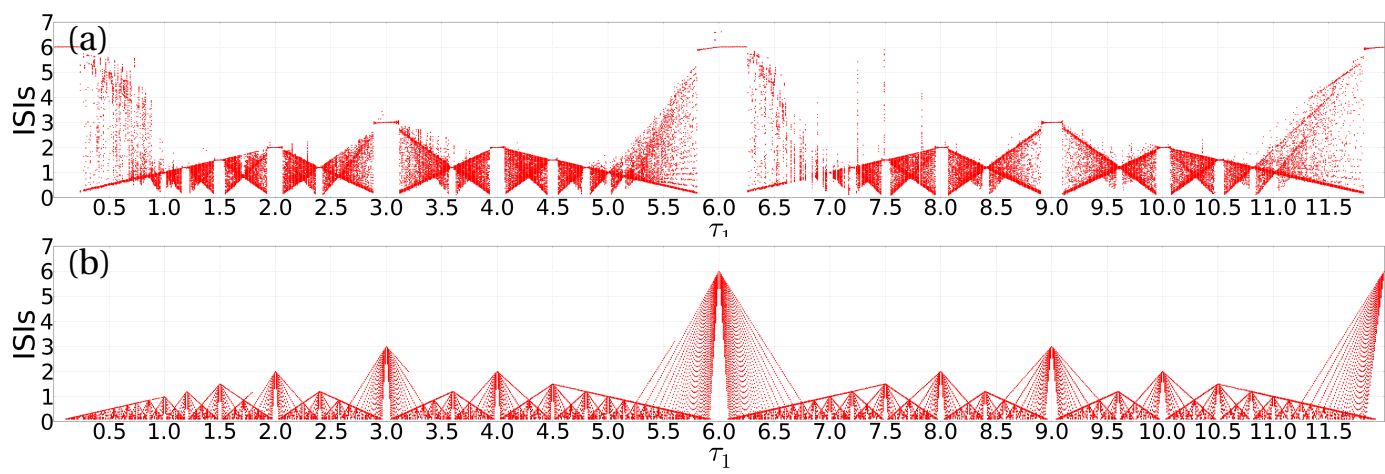

Figure 6.8.: Interspike intervals (ISIs) in a small-world network plotted $v$ s. the delay $\tau_{1}$ of the inner connections. (a) Simulations. (b) Mathematical reconstruction according to Eq. (6.1.3). $\tau_{2}=6, N=20, k=2, p=0.51$. Other parameters as in Fig. 6.1. Figure from [Cakan et al., 2014]. 


\subsubsection{Two discrete delay times}

In [Schöll et al., 2009; Panchuk et al., 2013] it was shown that already in a simple motif of two coupled FitzHugh-Nagumo systems with two or three different delay times, complex dynamics arise. In particular, resonance effects between the different delay times proved to be crucial. In Subsec. 5.1.5, we have employed this motif with two discrete delay time as the synchronization manifold to calculate whether the same dynamics also persists in large networks; see Fig. 5.4. Recall that a state of synchrony of $M$ groups moves on a synchronization manifold which corresponds to a motif of $M$ nodes. We have found for three exemplarily delay ratios that the dynamics - in this case zero-lag synchrony, antisynchronization, and bursting - are stable in larger networks when all links are excitatory.

We now want to extend this study by continuously varying the ratio of the two delay times where we simulate larger complex networks. We focus on small-world networks (see Fig. 6.7 for a schematic diagram) and separate the two parts of the network in a meaningful way by choosing $\tau_{2}$ as the delay associated with the underlying regular network (green arrows), and $\tau_{1}$ as the delay time of the additional random links (red arrows).

Depending on the ratio between $\tau_{1}$ and $\tau_{2}$, different spiking patterns emerge. We measure the ISIs (Interspike intervals) in simulations while gradually increasing $\tau_{1}$ for fixed $\tau_{2}=6$ as depicted in Fig. 6.8(a). Figure 6.8(b) shows a mathematical reconstruction of the results obtained numerically in panel (a) based on the following argument: Any spike in the network will eventually be fed into the system again with a delay. Starting from the synchronous manifold, i.e., $\left(u_{0}, v_{0}\right)=\ldots=\left(u_{N-1}, v_{N-1}\right) \equiv\left(u_{s}, v_{s}\right)$ (see also Sec. 3.1), spikes will first reappear with a delay of either $\tau_{1}$ or $\tau_{2}$. Those spikes again will be transmitted to other neurons with one of the two delays meaning that eventually nearly all possible combinations of forwarding a spike with either delay $\tau_{1}$ or $\tau_{2}$ will be observable in the network. Thus, we can obtain all possible spiking times from the expression:

$$
T_{l k}=l \tau_{1}+k \tau_{2}
$$

with $l, k \in \mathbb{N}_{0}$.

After sorting all possible $T_{l k}$ by size, the ISIs are given by the difference between neighboring elements in the sorted list. Note that for the mathematical reconstruction in Fig. $6.8(\mathrm{~b})$, ISIs $<0.1$ were discarded since the spikes have a width of approximately 0.1 if they are close, i.e., if the ISI is small. 
Coherent spiking, i.e., spiking with a constant ISI, is observable as a result of resonance effects: For a case where the ratio of the multiple delay times is given by

$$
n \tau_{1}=m \tau_{2}
$$

with $m, n \in \mathbb{N}$, coherent spiking with an ISI equal to

$$
\frac{\tau_{1}}{m}=\frac{\tau_{2}}{n}
$$

is induced, where we choose the smallest possible $m$ and $n$, i.e., $m$ and $n$ do not have common divisors. A similar relation has been obtained in [Zigzag et al., 2009] for a system of chaotic maps with several unequal delays and in [Panchuk et al., 2013] for two coupled FitzHugh-Nagumo systems with unequal coupling and self-feedback delay times. Note that $n$ and $m$ in Eq. (6.4) cannot be chosen arbitrarily large: If $\tau_{1} / m$ or $\tau_{2} / n$ is smaller than 0.4, spikes run into each other and coherent spiking is not possible any longer.

Due to the finite widths of the spikes, they show a locking behavior if the delays almost fulfill the resonance criterion given by Eq. (6.5). This yields the large locking tongues seen in Fig. 6.8(a) close to resonance delay times which are not visible in Fig. 6.8(b). Most clearly the resonance tongue is pronounced for $\tau_{1} \approx 6.0$ but can also be seen for $\tau_{1} \approx 2.0$, $\tau_{1} \approx 3.0, \tau_{1} \approx 8.0$, and $\tau_{1} \approx 9.0$, for example.

\subsubsection{Bimodal delay distributions}

In this Section, we discuss a network characterized by a bimodal delay distribution. In such a network, the superposition of two normal distributions with two mean delay times $\tau_{\mu}^{(1)}$ and $\tau_{\mu}^{(2)}$ and corresponding standard deviations $\sigma_{\tau}^{(1)}$ and $\sigma_{\tau}^{(2)}$ determines the delay between nodes.

\section{Peak distance of the distribution}

To study the effects of different bimodal distributions, we start by changing the difference $\tau_{\mu}^{(1)}-\tau_{\mu}^{(2)}$ between the two peaks of the distribution, while keeping the standard deviations constant: $\sigma_{\tau}^{(1)}=\sigma_{\tau}^{(2)}=0.01$. The result is shown in Fig. 6.9, where the ISIs are depicted vs. $\tau_{\mu}^{(1)}-\tau_{\mu}^{(2)}$. 


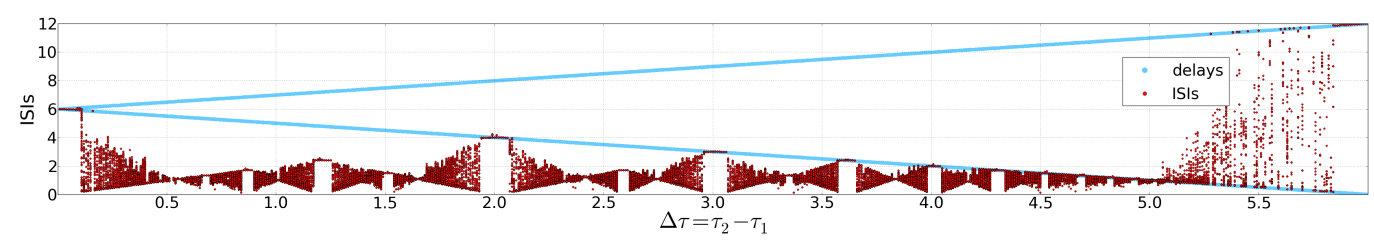

Figure 6.9.: Interspike intervals (ISIs) (red dots) and delays (blue dots) of a small-world network. $N=50, \sigma_{\tau}^{(1)}=\sigma_{\tau}^{(2)}=\sigma_{\tau}=0.01, p=0.51$. Other parameters as in Fig. 6.1. Figure from [Cakan et al., 2014].

Figure 6.9 shows that for distributions with small and medium width, i.e., $\sigma_{\tau}^{(1)}=\sigma_{\tau}^{(2)}<$ 0.01, the ISIs follow the condition of Eq. (6.4) discussed for the case of two discrete delay times if we substitute $\tau_{1}$ and $\tau_{2}$ by the two peak positions, i.e., $\tau_{1}=\tau_{\mu}^{(1)}$ and $\tau_{2}=\tau_{\mu}^{(2)}$. The same pattern also emerges for random networks.

\section{Width of the distribution}

If the width of the two peaks becomes too large, Eqs. (6.4) and (6.5) fail as good descriptions for the spike times and ISIs. Figure 6.10 depicts the ISIs as function of the peak widths $\sigma_{\tau}^{(1)}=\sigma_{\tau}^{(2)} \equiv \sigma_{\tau}$ for (a) a small world network with $\tau_{\mu}^{(1)}=6$ and $\tau_{\mu}^{(2)}=8$, (b) a small world network with $\tau_{\mu}^{(1)}=5$ and $\tau_{\mu}^{(2)}=10$, and (c) a regular ring with $\tau_{\mu}^{(1)}=6$ and $\tau_{\mu}^{(2)}=8$. For small $\sigma_{\tau}\left(\sigma_{\tau}<0.05\right.$ in panel (a), $\sigma_{\tau}<0.09$ in panel (b), and $\sigma_{\tau}<0.03$ in panel (c)) the ISIs can be found by evaluating Eq. (6.5): For the combination $\tau_{\mu}^{(1)}=6$ and $\tau_{\mu}^{(2)}=8$, we find coherent spiking with an ISI of 2 , for $\tau_{\mu}^{(1)}=5$ and $\tau_{\mu}^{(2)}=10$ the ISI is 5 .

As $\sigma_{\tau}$ increases, the coherent spiking breaks down. Instead, networks can be observed where different parts of the network spike with different ISIs. This effect is particularly prominent in the case of a ring network, since in such a network isolated subnetworks can easily arise, in which the delay distribution allows for persistent spiking. Figure 6.11 shows exemplarily the dynamics in a regular ring network for intermediate values of the distribution width $\left(\sigma_{\tau}=0.05\right.$ in panels (a),(b),(c), $\sigma_{\tau}=0.08$ in panels (d),(e),(f)). Panels (c) and (f) show the spiking patterns; each dots marks the spiking of a node. For the lower $\sigma_{\tau}$ value, shown in panel (c), a part of the network (from about node 30 to node 45 ) exhibits partial amplitude death, while the majority of the nodes keeps spiking, though in different subnetworks characterized by different ISIs. For the higher value of $\sigma_{\tau}$, only a small subset of spiking nodes persists. The time series of node 0 in panel (a) and its 


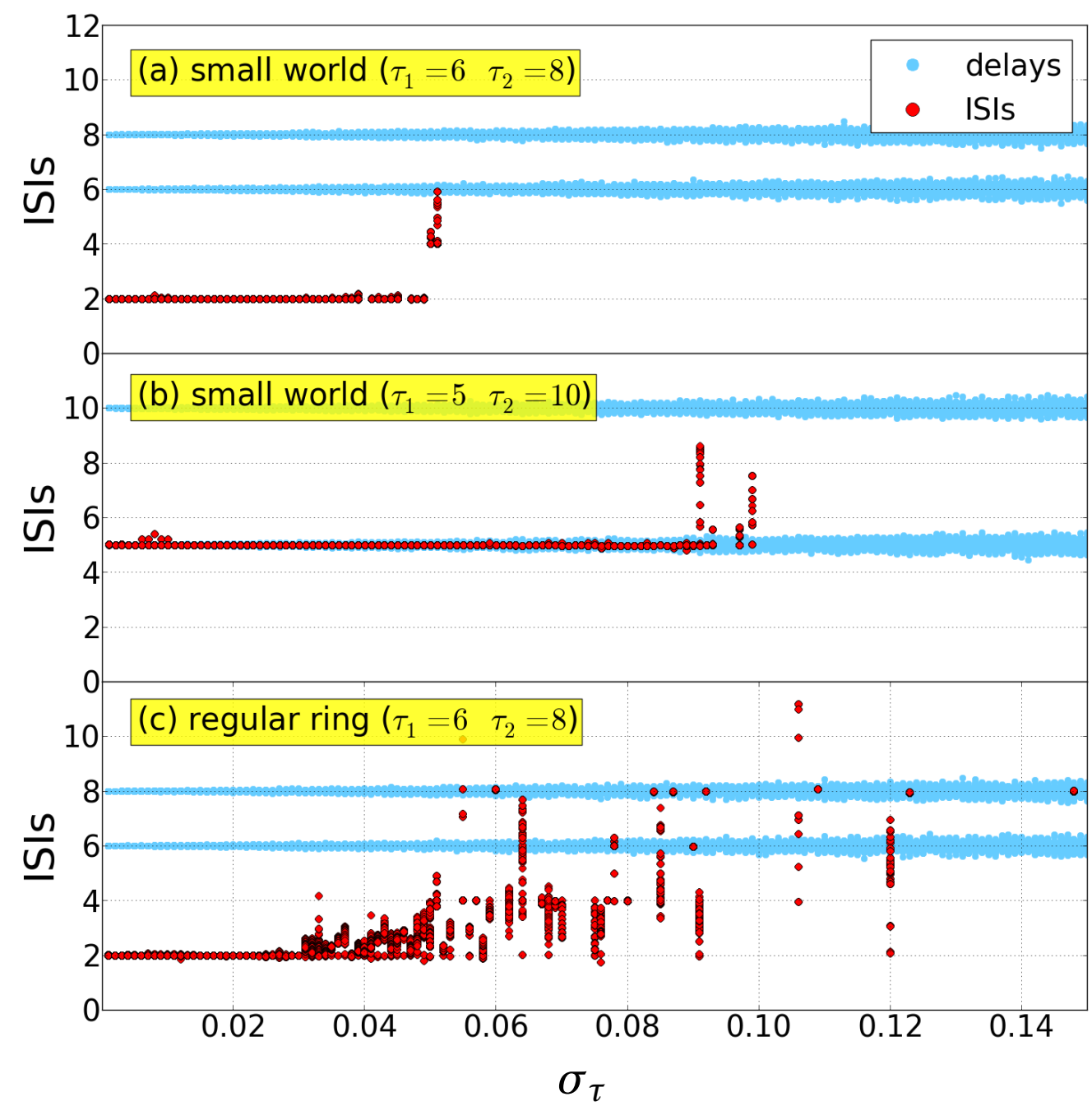

Figure 6.10.: Interspike intervals (ISIs) (red dots) and delay distributions (blue dots) vs. $\sigma_{\tau} \equiv \sigma_{\tau}^{(1)}=\sigma_{\tau}^{(2)}$ for different combinations of mean delay times. (a), (b): small-world network $(k=2, p=0.51),(c)$ : regular ring network $(k=2)$. Mean delay times are $\tau_{1}=6$ and $\tau_{2}=8$ for (a) and (c), and $\tau_{1}=5$ and $\tau_{2}=10$ for (b). $N=50$. Other parameters as in Fig. 6.1. Figure from [Cakan et al., 2014]. 

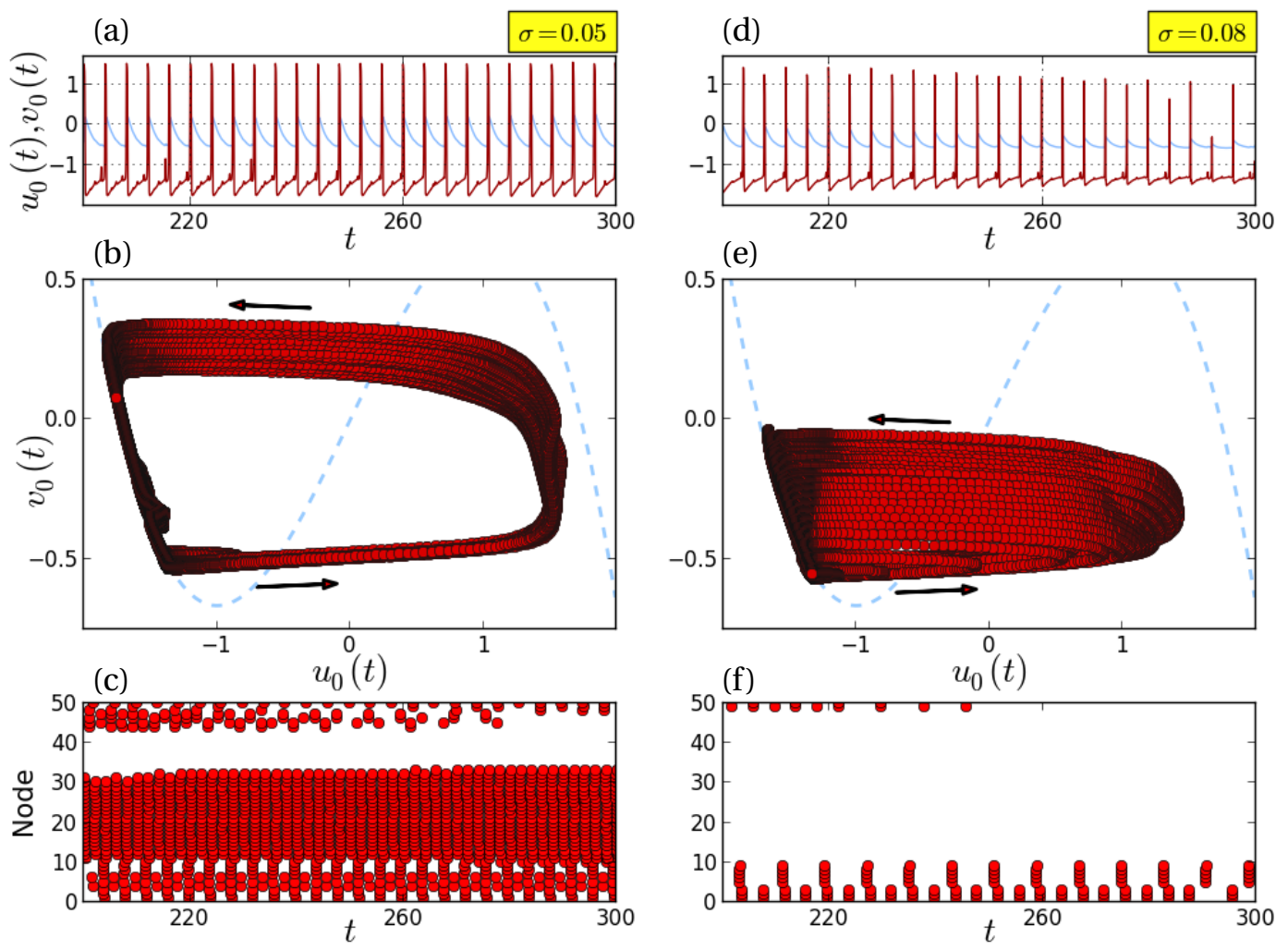

Figure 6.11.: Dynamics for a bimodal delay distribution in a regular ring with $k=2$ for different $\sigma_{\tau}$ : (a)-(c) $\sigma_{\tau}=0.05 ;(d)-(f) \sigma_{\tau}=0.08$. (a) and (d): time series of $u_{0}$ (dark red) and $v_{0}$ (light blue); (b) and (e): phase portraits of a single spiking node $\left(u_{0}, v_{0}\right)$ : trajectory (red solid) and u-nullcline (blue dashed); (c) and (e): spiking patterns. Mean delay times $\tau_{1}=6, \tau_{2}=8$. Other parameters as in Fig. 6.1. Figure from [Cakan et al., 2014]. 
phase portrait in (b) show that no longer all spikes have the same amplitude, but the amplitudes vary slightly in an irregular fashion due to the coupling with other nodes with inhomogeneous delay times. If $\sigma_{\tau}$ is further increased, global amplitude death sets in.

How robust the network is towards increasing the peak widths, depends on the topology and the mean delay times. If $m$ and $n$ in Eq. (6.5) are large, coherent spiking is less robust, since the probability that spikes overlap constructively after a time $n \tau_{\mu}^{(1)}=m \tau_{\mu}^{(2)}$ decreases. For example, in the case $\tau_{\mu}^{(1)}=6$ and $\tau_{\mu}^{(2)}=8, n=3$ and $m=2$, while $\tau_{\mu}^{(1)}=5$ and $\tau_{\mu}^{(2)}=10$ yield the combination $n=2$ and $m=1$, explaining why the synchronized spiking collapses in Fig. 6.10(a) for smaller $\sigma_{\tau}$ than in Fig. 6.10(b). The size of the interval of $\sigma_{\tau}$ in which asynchronous spiking takes place, depends on the topology. In regular rings the interval is considerably larger (see Fig. 6.10(c)), because subnetwork synchronization, can occur as already observed in unimodal delay distributions. This subnetwork synchronization causes ISIs different than the ones predicted using Eq. (6.4) while the spiking can still remain regular. In random networks (not shown here), the interval shrinks to zero as no regularity is left in the topology.

\subsection{Cluster STATES IN CHEMICAL OSCILLATORS}

In this Section, I will discuss experiments on chemical oscillators, which can, in certain regimes of operation, be mathematically described by simple models like Kuramoto phase oscillators or Stuart-Landau oscillators. Choe et al. [2010] derived stability and existence criteria for cluster states in delay-coupled Stuart-Landau oscillators as has been recapitulated in Sec. 5.2. Their results suggest that such states might also be observable in coupled chemical oscillators. This section discusses the application of their theory to cluster states in chemical oscillators and is based on [Blaha et al., 2013].

In regimes where the oscillators are not well described by the Stuart-Landau oscillator with a linear coupling but are of relaxational type an extension of this theory is needed which will be discussed in Subsec. 6.2.3.

\subsubsection{Experimental setup}

The experiments were carried out by Karen Blaha in the group of John Hudson. They were performed in an electrochemical cell consisting of four 1-mm diameter nickel working electrodes (99.98\% pure), a Platinum mesh counter electrode, and $\mathrm{Hg} / \mathrm{Hg}_{2} \mathrm{SO}_{4} / \mathrm{K}_{2} \mathrm{SO}_{4}$ 
(sat) reference electrode, with a $3 \mathrm{M} \mathrm{H}_{2} \mathrm{SO}_{4}$ electrolyte; see Fig. 6.12(a). The cell is enclosed in a jacketed glass vessel maintained at a temperature of $11^{\circ} \mathrm{C}$. The working electrodes are the network nodes and are coupled in a unidirectional ring. A resistor, $R_{p}=$ $650 \Omega$, is attached to each of the electrodes. An ACM Instruments multi-channel potentiostat is used to keep the potentials $V_{0}$ of the electrodes such that they undergo transpassive dissolution. The resulting electrodissolution currents are measured at $250 \mathrm{~Hz}$ using zero resistance amperemeters (ZRAs) attached to a real time data acquisition system.

In the transpassive state, $V_{o}$ is sufficiently high that the nickel ions are capable of penetrating the oxide layer accumulated at the electrode [Cioffi et al., 2011; Blaha, 2013]. The transpassive state follows the passive state where $V_{0}$ is high enough to oxidize the electrode but too weak to enable nickel ions to overcome this oxide layer; thus, in the passive state the current is zero, while it grows approximately linearly with $V_{0}$ in the transpassive state if no resistor is attached. The active state precedes the passive state; here $V_{0}$ is too low for oxidation. The transpassive state of nickel electrodissolution is known for its rich nonlinear dynamics [Cioffi et al., 2011]. Here, parameters are chosen such that the attached resistors induce oscillations in $I_{j}$ via a Hopf bifurcation [Haim et al., 1992].

This Hopf bifurcation occurs at approximately $V_{0} \approx 1.05 \mathrm{~V}$. Closely above the Hopf bifurcation the oscillators' phase dynamics can be modeled as sinusoidal oscillations [Kiss et al., 2005], even though the time series is not perfectly sinusoidal (see Fig. 6.12(c)). Thus, depending on the choice of applied voltage two different operating regimes can be distinguished in the uncoupled nodes:

(i) Low voltages induce nearly-harmonic oscillations (for a time series see Fig. 6.12(b)), which can be modeled by Stuart-Landau oscillators.

(ii) For high voltages the oscillations exhibit higher harmonics as can be seen in the time series in Fig. 6.12(c) and the Stuart-Landau oscillator with linear coupling fails as a good model.

We will use the terms (i) smooth and (ii) relaxation oscillations to distinguish these two regimes and will discuss the application of the theory to each oscillation type in Subsecs. 6.2.2 and 6.2.3, respectively.

Four oscillators with similar frequencies are selected from an array of 64 oscillators. Interactions are introduced via the using real-time coupling of the form

$$
V_{j}(t)=V_{0}+\delta V_{j}(t)
$$



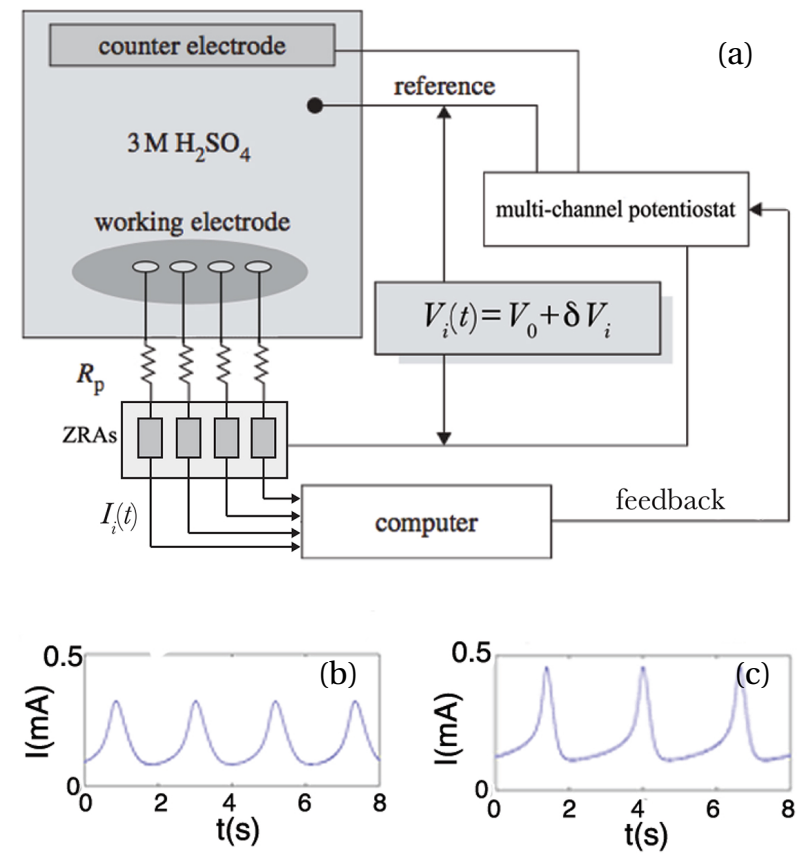

Figure 6.12.: Experimental set up and time series of electrochemical oscillations. (a) Experimental apparatus with multi-channel addressable feedback, $R_{p}$ is the channel resistance of $650 \Omega$. ZRA: zero resistance amperemeter. (b) Electrochemical dissolution time series showing smooth oscillators at a potential of $V_{0}=1.105 \mathrm{~V}$. (c) Time series of relaxation oscillators at $V_{0}=1.2$ V. Figure from [Blaha et al., 2013]. 
where $\delta V_{j}$ are the changes in the circuit potentials of the $j$ th element due to the feedback. These feedback voltages are given by

$$
\delta V_{j}(t)=K{ }_{n=1}^{N} G_{j n} V_{n}(t-\tau)-R_{p} \hat{I}_{n}(t-\tau)
$$

where $R_{p}=650 \Omega$ is the channel resistance, $K$ is the fixed overall coupling gain, and $\tau$ denotes the coupling time delay, which is realized by the real-time data acquisition system combined with the multi-channel potentiostat. $\mathbf{G}$ is the coupling matrix, i.e., in the following a unidirectional ring, i.e., $G_{i j}=\delta_{(i+1) \bmod N, j}$; see also Eq. (2.16). This coupling scheme is implemented via the multi-channel potentiostat (see Fig. 6.12). $\hat{I}_{n}$ are the normalized currents measured by the ZRAs and calculated as

$$
\hat{I}_{n}(t)=\frac{I_{j}^{\max }}{\left\langle I_{i}^{\max }\right\rangle_{i}}\left(I_{j}(t)-\left\langle I_{j}\right\rangle_{t}\right),
$$

where $I_{j}^{\max }$ is the amplitude of oscillator $j$ and $\left\langle I_{i}^{\max }\right\rangle_{i}$ is the mean amplitude of all oscillators. $\left\langle I_{j}\right\rangle_{t}$ is the time average of the $j$ th channel [Blaha, 2013].

\subsubsection{Smooth oscillations}

In this Subsection, we discuss to what extent the theory of Choe et al. [2010] (see Sec. 5.2) can predict the experimental results in the smooth regime.

As a model for the smooth oscillations, we use the Stuart-Landau oscillator with a realvalued coupling strength. For a unidirectional ring, the equations are given by

$$
\dot{z}_{j}=\left[\lambda+\mathrm{i} \omega-\left|z_{j}\right|^{2}\right] z_{j}+K z_{(j+1) \bmod N, \tau},
$$

where $z_{j} \in \mathbb{C}$. $\lambda$ and $\omega$ are the bifurcation parameter and the frequency of an uncoupled $(K=0)$ node, respectively; see also Eq. (5.46) and its description. Note that we here use - in contrast to [Choe et al., 2010] and in Sec 5.2 - a non-diffusion like coupling. There, the coupling term reads $z_{j+1}(t-\tau)-z_{j}$ instead of just $z_{j+1}(t-\tau)$. Nevertheless, this alters the involved equations only slightly such that we only have to adapt the analysis of [Choe et al., 2010] at a few points. Equation (6.9) can be rewritten in amplitude and phase 
variables with $r_{j}=\left|z_{j}\right|$ and $\varphi_{j}=\arg \left(z_{j}\right)$ :

$$
\begin{aligned}
\dot{r}_{j}(t) & =\lambda-r_{j}^{2} r_{j}+K r_{(j+1) \bmod N, \tau} \cos \varphi_{(j+1) \bmod N, \tau}-\varphi_{j}, \\
\dot{\varphi}_{j}(t) & =\omega+K \frac{r_{(j+1) \bmod N, \tau}}{r_{j}} \sin +\varphi_{(j+1) \bmod N, \tau}-\varphi_{j},
\end{aligned}
$$

where $i=0, \ldots, N-1$.

We calculate the dynamical variables (amplitudes $r_{j}$ and phases $\varphi_{j}$ ) from the ZRAs' experimental measurement of the electrodissolution current of each oscillator; see Fig. 6.12. From these currents, the phase of each oscillator is found by peak-to-peak linear interpolation, where the peak is defined as 0 or $2 \pi$ [Rusin et al., 2010]. From the phases, we can then calculate the average frequencies of the oscillators. The amplitudes are measured as half of the difference between the peak and trough value of the electrodissolution current, giving one data point per period.

The parameters $\lambda$ and $\omega$ of the theoretical model can be identified by the dynamics of a single uncoupled oscillator (see Figs. 6.12(b) and (c)): $\lambda=\sqrt{r}$, where $r$ is the amplitude of the uncoupled oscillations, while $\omega=2 \pi / T$, where $T$ is the period of its oscillation. In the experiments, the oscillators have slightly different frequencies; we, thus, use for $\omega$ the average frequency, i.e., $\omega=\left\langle\omega_{j}\right\rangle$.

For the experiment, the four electrodes were held at a voltage of $V_{0}=1.105 \mathrm{~V}$, slightly above the Hopf bifurcation. The four oscillators operate at $\omega_{0}=3.424 \pm 0.063 \mathrm{rad} / \mathrm{s}, \omega_{1}=$ $3.393 \pm 0.069 \mathrm{rad} / \mathrm{s}, \omega_{2}=3.418 \pm 0.063 \mathrm{rad} / \mathrm{s}$, and $\omega_{3}=3.456 \pm 0.057 \mathrm{rad} / \mathrm{s}$ meaning that the nodes are slightly non-identical. The frequency range is due to the slow drift of the natural frequencies of the oscillators.

We now want to predict the occurrence of cluster states in dependence upon $\tau$. The primary states which we discuss in this Section are described by

$$
\begin{aligned}
r_{j} & =r_{0, m}, \\
\varphi_{j} & =\Omega_{m} t+\frac{2 \pi m}{N} j, \quad j=0, \ldots, N-1 .
\end{aligned}
$$

Thus, these states are characterized by equal radii and equal phase differences. This is in contrast to the secondary states which we will investigate in Subsecs. 6.2.3 and 6.2.4. 

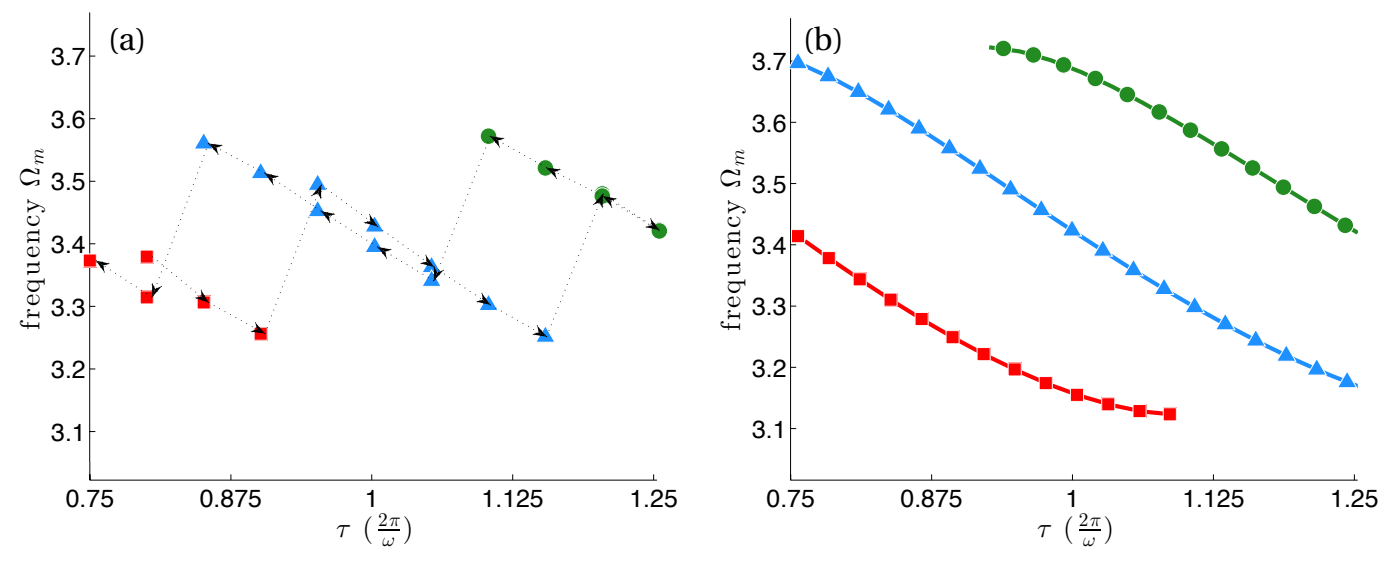

Figure 6.13.: Collective frequency $\Omega_{m}$ versus the time delay $\tau$ for the smooth oscillations. Red squares, blue triangles, and green circles represent a reverse splay state, an inphase state, and a splay state, respectively. (a) Experimental data, $V_{0}=1.105 \mathrm{~V}$, $K=0.15$. The arrows indicate an increase or decrease of $\tau$ during the measurement. (b) Solution continuation of Eq. (6.10) using DDE-BIFTOOL (symbols) and stable solutions of Eq. (6.12) (lines) where the stability is calculated according to Eq. (6.13). Parameters: $\lambda=1.1025, \omega=3.4228, K=0.3, N=4$. Figure from [Blaha et al., 2013]. 
The integer $m=0, \ldots, N-1$ labels the specific states: In the case of four nodes, $m=0$ corresponds to zero-lag synchronization, $m=1$ is the splay state, $m=2$ the 2-cluster state, while $m=3$ labels the reverse splay state (see the lower panels of Fig 6.14 for a schematic depiction). Applying this notion to Eqs. (6.10) yields the following set of transcendental equations for the collective amplitude $r_{0, m}$ and frequency $\Omega_{m}$ of the $m$-state:

$$
\begin{aligned}
& r_{0, m}^{2}=\lambda+K \cos \Phi_{m}, \\
& \Omega_{m}=\omega+K \sin \Phi_{m},
\end{aligned}
$$

where $\Phi_{m}=2 \pi m / N-\Omega_{m} \tau$.

The Floquet exponents describing the time evolution of a small perturbation from the synchronized periodic state (6.11) are given by the eigenvalues $\Lambda$ of the characteristic equation

$$
\operatorname{det} \mathbf{J}_{0, m}-\Lambda \mathbf{I}_{2}+K-1+e^{2 \mathrm{i} k \pi / N-\Lambda \tau} \mathbf{R}_{m}=0,
$$

where $\mathbf{I}_{N}$ is $N \times N$ identity matrix ,

$$
\mathbf{R}_{m}=\begin{array}{cc}
\cos \Phi_{m} & -\sin \Phi_{m} \\
\sin \Phi_{m} & \cos \Phi_{m}
\end{array},
$$

and the Jacobian of the local dynamics is given by

$$
\mathbf{J}_{0, m}=\begin{array}{cc}
-2 r_{0, m}^{2} & 0 \\
0 & 0
\end{array}
$$

If for all $k=0, \ldots, N-1$ all Floquet exponents (except the one relating to the Goldstone mode) have a negative real part, the cluster state with index $m$ will be stable.

Figure 6.13 depicts the measured and numerically calculated stable states of the compound system depending upon the time delay in panels (a) and (b), respectively. In Fig. 6.13(b), the lines are calculated from Eq. (6.12) where we only consider solutions which are stable according to Eq. (6.13). The points are based on solution continuation of Eq. (6.10) using DDE-BIFTOOL. In order to resolve the multistability present in the coupled system we slowly increase the time delay (shown by arrows in Fig. 6.13(a)) up to $\tau=1.25 \times(2 \pi / \omega)$ at which point we perform a down-ramping. During the up-sweep, $\tau$ is increased from $0.80 \times(2 \pi / \omega)$ to $1.25 \times(2 \pi / \omega)$ in increments of $0.05 \times(2 \pi / \omega)$. The system is allowed to reach a stationary state at each value of $\tau$. The qualitatively differ- 
ent states are marked by the following symbols: red squares represent the reverse splay state, blue triangles represent the in-phase state, and green circles represent the splay state.

We start at $\tau=0.8 \times(2 \pi / \omega)$ with a reverse splay state, which is characterized by a phase difference of $3 \pi / 2$ between two subsequent oscillators. Increasing to $\tau=0.95 \times(2 \pi / \omega)$, we obtain in-phase synchronization with $\varphi_{0}=\varphi_{1}=\varphi_{2}=\varphi_{3}$. For larger $\tau$ values, the system exhibits a splay state.

During the down-sweep, the time delay is decremented by $0.05 \times(2 \pi / \omega)$ until $\tau=0.75 \times$ $(2 \pi / \omega)$. We observe the same states in the down-sweep as the up-sweep. If no cluster transition occurs, the change in frequency is continuous (as expected from Eq. (6.12)), while at a cluster transition it abruptly jumps to a lower value. The system maintains the splay state until transitioning to the in-phase cluster state and then the reverse splay state. The transitions are also shown by arrows for the up- and down-sweep. Note that they occur at different time delays depending upon the direction of the time delay sweep. The coexistence of several cluster states at a given value of $\tau$ demonstrates hysteresis. In Fig. 6.13(a) showing the experimental data, the triangles representing the in-phase cluster state are slightly nonidentical near $\tau=1 \times(2 \pi / \omega)$; this is due to drift in the natural frequencies during the course of the experiment.

Figures 6.14(a)-(d) illustrate the qualitative differences between the cluster states seen in Fig. 6.13. Below the time series, corresponding schematic diagrams are also depicted. Note that the 2-cluster state (Fig. 6.14(b)) does not occur in the range of $\tau$ shown in Fig. 6.13.

In conclusion, the cluster states and hysteresis observed in the experiments can be modeled by the Stuart-Landau oscillator as given in Eq. (6.10). The numerical results, including simulations as well as path continuation using DDE-BIFTOOL [Engelborghs et al., 2001, 2002], shown in Fig. 6.13(b) closely match the experimental results. The only discrepancy seen is that the branches of each cluster state in the experiments seem to be stable for a shorter range of $\tau$, leading to an earlier jump to another cluster state. This is probably due to experimental noise and small heterogeneities in the oscillators' parameters.

\subsubsection{Relaxation oscillations}

If the voltage is increased to $V_{0}=1.2 \mathrm{~V}$, the profile of the oscillations deforms from a smooth to a strongly nonlinear relaxation oscillation. A nonlinear time transformation can be used to map the relaxation oscillations back to the smooth model such that the 

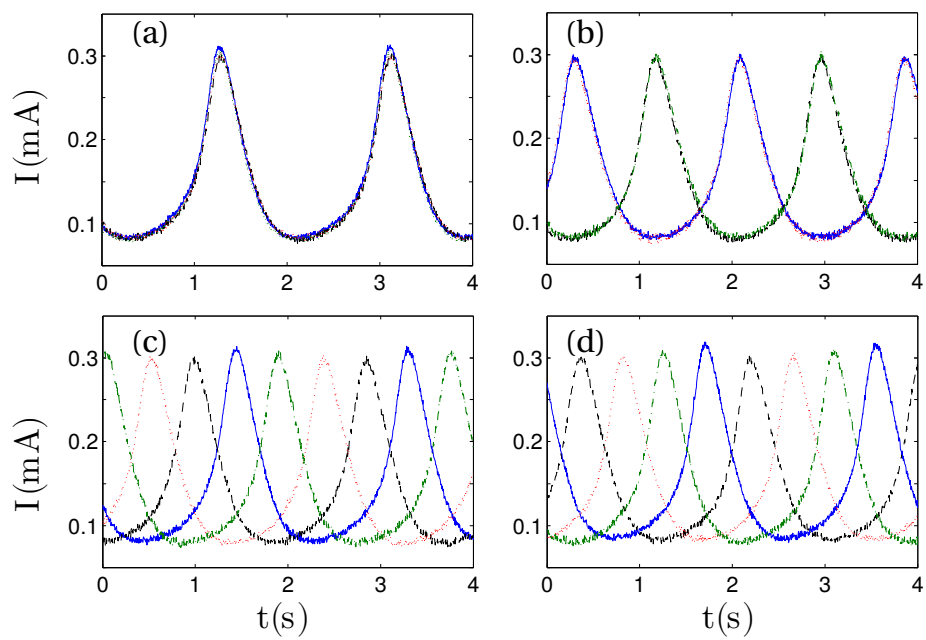

(a)

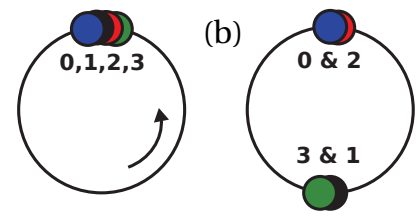

(c)

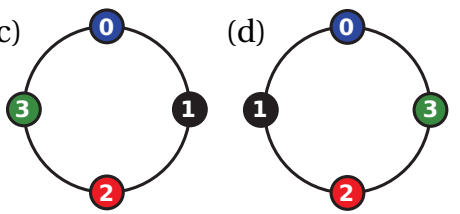

Figure 6.14.: Experimental time series (top) and schematic diagram (bottom) of an (a) inphase state, (b) a 2-cluster state, (c) a reverse splay state, and (d) a splay state as shown in Fig. 6.13(a). The schematics show phase relations between oscillators on the phase ring. Oscillator colors in the schematic correspond to the colors in the times series: $\varphi_{0}$ is shown in blue, $\varphi_{1}$ is black, $\varphi_{2}$ is red, and $\varphi_{3}$ is green. Parameters: $V_{0}=1.105 \mathrm{~V}$, $K=0.15$; time delays: (a) $\tau=1.05 \times 2 \pi / \omega$, (b) $0.5 \times 2 \pi / \omega$, (c) $1.2 \times 2 \pi / \omega$, and (d) $0.8 \times 2 \pi / \omega$ with $\omega=3.4228 \mathrm{rad} / \mathrm{s}$. Figure from [Blaha et al., 2013]. 
Stuart-Landau model can still be employed for a theoretical description. However, this nonlinear time transformation will also affect the coupling, which no longer can be assumed to be smooth. Instead we rewrite Eq. (6.10) in a more general form

$$
\begin{aligned}
& \dot{r}_{j}=\left(\lambda-r_{j}^{2}\right) r_{j}+K r_{j+1}(t-\tau) H_{r}\left(\varphi_{j+1}(t-\tau)-\varphi_{j}(t)\right), \\
& \dot{\varphi}_{j}=\omega+K \frac{r_{j+1}(t-\tau)}{r_{j}} H_{\varphi}\left(\varphi_{j+1}(t-\tau)-\varphi_{j}(t)\right),
\end{aligned}
$$

where $H_{r}$ and $H_{\varphi}$ are coupling functions, also called interaction functions, which can be obtained experimentally. For details of the corresponding experiment see [Blaha et al., 2013].

Figure 6.15 shows the experimentally measured interaction functions $H_{r}$ and $H_{\varphi}$ for the low voltage $V_{0}=1.105 \mathrm{~V}$ in panels (a) and (c) and for the higher voltage $V_{0}=1.2 \mathrm{~V}$ in panels (b) and (d). The black curve is a 5th-order Fourier fit from evenly sampled data. For $V_{0}=1.105 \mathrm{~V}$ (panels (a) and (c)), the radial interaction function remains approximately constant and the phase interaction function exhibits a sinusoidal shape. Thus, we have $H_{\varphi}(\Delta \varphi)=\sin (\Delta \varphi)$ as considered in Eq. (6.10).

At this point, we also see a possible cause for the difference between Fig. 6.13(a) and (b). The frequencies calculated from Eq. (6.11) rely on the use of an appropriate phase interaction function. The radial interaction function does not influence the frequencies, but it does influence the stability of the particular state according to Eq. (6.13). In Eqs. (6.11) and (6.13), we used a cosine for the radial interactions while in the experiment the radial coupling is approximately constant as just discussed. The lack of an appropriate radial interaction function might explain the difference between the stability of the states shown in Fig. 6.13(a) and (b).

For $V_{0}=1.2 \mathrm{~V}$, both interaction functions have a more complex structure. In order to take their complex shapes into account in our theory, we approximate $H_{r}$ and $H_{\varphi}$ by the Fourier series. Thus, Eq. (6.16a) reads:

$$
\begin{gathered}
\dot{r}_{j}=\lambda-r_{j}^{2} r_{j}+K{ }_{n=0}^{N-1} a_{j n} r_{n, \tau}{ }_{l=0}^{5} a_{l, r} \cos l\left(\varphi_{n, \tau}-\varphi_{j}\right)+b_{l, r} \sin l\left(\varphi_{n, \tau}-\varphi_{j}\right) \\
\dot{\varphi}_{j}=\omega+K_{n=0}^{N-1} a_{j n} \frac{r_{n, \tau}}{r_{n}}{ }_{l=0}^{5} a_{l, \varphi} \cos l\left(\varphi_{n, \tau)}-\varphi_{j}\right)+b_{l, \varphi} \sin l\left(\varphi_{n, \tau}-\varphi_{j}\right)
\end{gathered}
$$




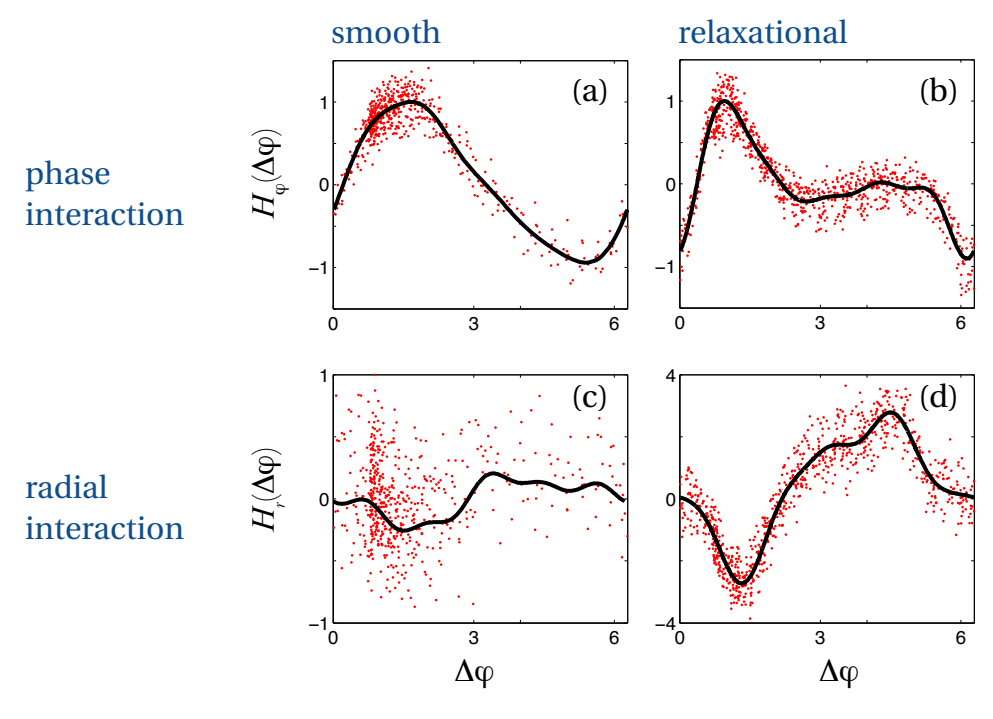

Figure 6.15.: Phase interaction function $H_{\varphi}$ in panels (a) and (b) for $V_{0}=1.105 \mathrm{~V}$ and $V_{0}=$ $1.2 \mathrm{~V}$, respectively; radial interaction function $H_{r}$ in panels (c) and (d) for $V_{0}=1.105 \mathrm{~V}$ and $V_{0}=1.2 \mathrm{~V}$, respectively. Experimentally obtained data is shown as red dots. A 5term Fourier fit from evenly sampled data is shown by the black curve. Figure from [Blaha et al., 2013].

where the Fourier coefficients $a_{l, r}, b_{l, r}, a_{l, \varphi}$, and $b_{l, \varphi}$ are determined by a fit to the experimentally obtained interaction functions $H_{r}$ and $H_{\varphi}$. The coefficients are given in Tab. 6.1. They are normalized such that $\max \left|H_{\varphi}\right|=1$. Thus, the coupling strength $K$ still represents the overall coupling strength. An extension of the theory of Choe et al. [2010] to system (6.17) will be given in Subsec. 6.2.4. In the remaining part of this Section the dependency of the frequency upon the delay time is investigated experimentially and with the help of DDE-BIFTOOL.

The oscillators for these experiments now have a different mean intrinsic frequency than in Subsec. 6.2.2. For the experiment yielding the results seen in Fig. 6.16 the four oscillators operate at $\omega_{0}=2.421 \pm 0.068 \mathrm{rad} / \mathrm{s}, \omega_{1}=2.445 \pm 0.088 \mathrm{rad} / \mathrm{s}, \omega_{2}=2.407 \pm 0.069 \mathrm{rad} / \mathrm{s}$, and $\omega_{3}=2.449 \pm 0.112 \mathrm{rad} / \mathrm{s}$.

The relaxation oscillators exhibit more complicated cluster and hysteresis behavior as depicted in Figs. 6.16(a) and (b) for experimental and numerical data, respectively. For the latter we use the continuation software DDE-BIFTOOL similar to the case of smooth oscillators. The lines in Figs. 6.16(b) will be explained in Subsec. 6.2.4. The states detected with DDE-BIFTOOL agree very well with the experimental results in Fig. 6.16(a), 


\begin{tabular}{|c|c|}
\hline \multicolumn{2}{|c|}{ Radial interaction function } \\
\hline$a_{1, r}=-0.97948$ & $b_{1, r}=-1.82354$ \\
\hline$a_{2, r}=0.36110$ & $b_{2, r}=-0.07963$ \\
\hline$a_{3, r}=0.29724$ & $b_{3, r}=0.54854$ \\
\hline$a_{4, r}=0.05846$ & $b_{4, r}=0.09098$ \\
\hline$a_{5, r}=-0.11558$ & $b_{5, r}=-0.09251$ \\
\hline \multicolumn{2}{|l|}{$a_{0, r}=0.45579$} \\
\hline \multicolumn{2}{|c|}{ Angular interaction function } \\
\hline$a_{1, \varphi}=-0.00610$ & $b_{1, \varphi}=0.31622$ \\
\hline$a_{2, \varphi}=-0.35811$ & $b_{2, \varphi}=0.29020$ \\
\hline$a_{3, \varphi}=-0.25341$ & $b_{3, \varphi}=-0.05585$ \\
\hline$a_{4, \varphi}=-0.13541$ & $b_{4, \varphi}=0.00799$ \\
\hline$a_{5, \varphi}=-0.07183$ & $b_{5, \varphi}=0.00425$ \\
\hline$a_{0, \varphi}=0$ & \\
\hline
\end{tabular}

Table 6.1.: Fourier coefficients of the coupling function used in Eq. (6.17). 

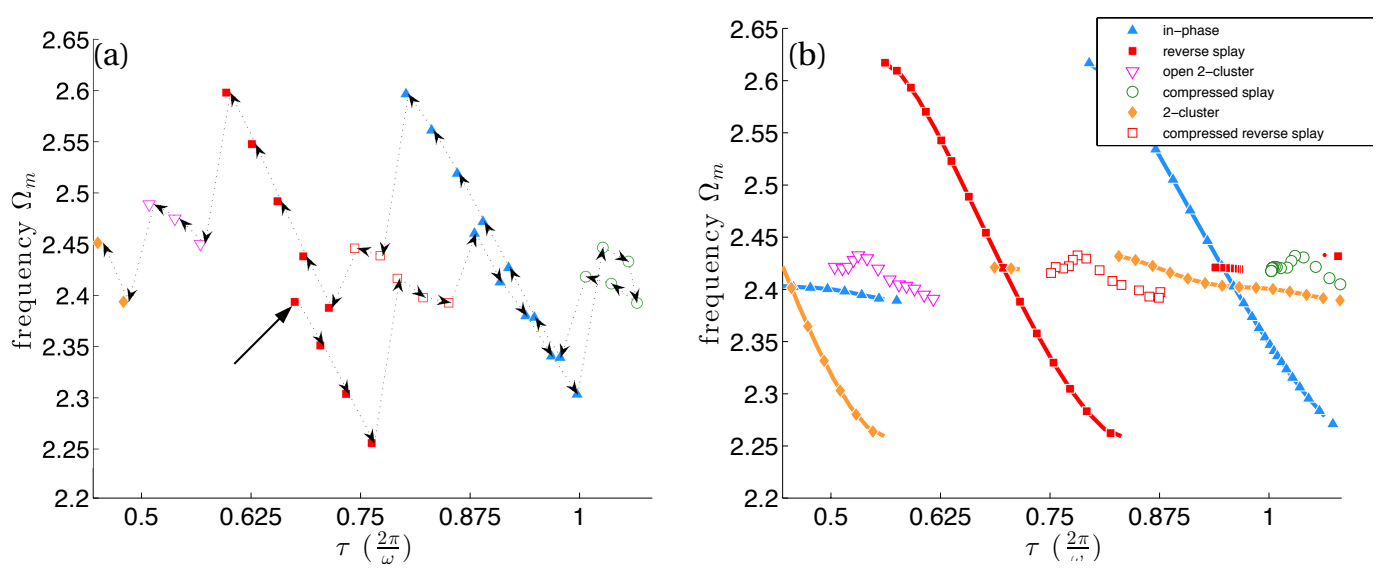

Figure 6.16.: Dynamics in dependence on the time delay $\tau$ for the relaxation oscillations. (a) Experimental data, $V_{0}=1.2 \mathrm{~V}, \mathrm{~K}=0.10$. The starting state is marked by a black arrow. The increasing and decreasing of $\tau$ during the experiment is shown with arrows. (b) Solutions of Eq. (6.17) using the continuation tool DDE-BIFTOOL (markers) and stable solutions of Eq (6.19) (lines) where the stability is given by Eq. (6.25). In-phase, 2-cluster, compressed splay, reverse splay, compressed reverse splay and open 2-cluster states are represented by upward-triangles, diamonds, open circles, squares, open squares and downward-triangles, respectively. The interaction functions $H_{r}$ and $H_{\varphi}$ are chosen as in Eq. (6.17) and Tab. 6.1. Parameters: $\lambda=2.890$, $K=0.189$ and $\omega=2.430$. Figure from [Blaha et al., 2013]. 

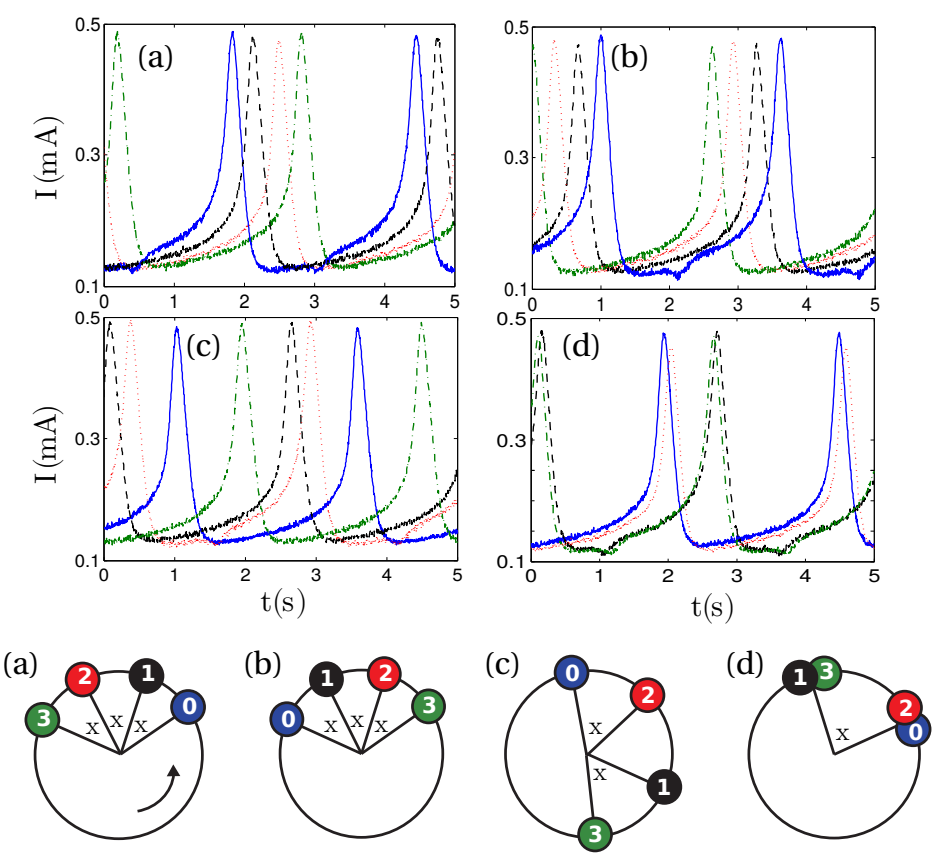

Figure 6.17.: Experimental time series (top) and schematic diagram (bottom) of secondary states: (a) a compressed splay state, (b) a reverse compressed splay state, (c) an open 2-cluster state, (d) a compressed 2-cluster state. Parameters: $V_{0}=1.2 \mathrm{~V}, \mathrm{~K}=0.10$; time delays: (a) $\tau=1.11 \times 2 \pi / \omega$, (b) $0.82 \times 2 \pi / \omega$, (c) $0.59 \times 2 \pi / \omega$ with $\omega=2.43 \mathrm{rad} / \mathrm{s}$ as in Fig. 6.16 and $(d) 0.68 \times 2 \pi / \omega$ with $\omega=2.492 \mathrm{rad} / \mathrm{s}$. Figure from [Blaha et al., 2013].

where the black arrow marks the starting configuration. Note that only stable solutions relevant to the experimental results are shown. For the comparison between Figs. 6.16(a) and (b), it must also be noted that while the natural frequency $\omega$ varies slowly during the experiments due to drift, it is kept constant in the numerical calculations. A sequence of different cluster states occurs as the time delay $\tau$ is increased or decreased. The primary states discussed earlier in Fig. 6.14, (in-phase, 2-cluster, reverse splay and splay states) are also present in the case of the relaxational oscillators. However, in the current regime of operation additional qualitatively different states are possible, which we will refer to as secondary states.

To illustrate the secondary states, Fig. 6.17 shows several experimental time series and corresponding schematic diagrams. Figure 6.17(a) depicts a compressed splay state. As in the splay state, $\varphi_{3}-\varphi_{2}=\varphi_{2}-\varphi_{1}=\varphi_{1}-\varphi_{0}$, but these phase differences (marked by $x$ in the schematic diagram) are different from a multiple of $\pi / 2$. Recall that in the case of the 
primary states the phase differences between the clusters are equal and given by $2 \pi / M$ where $M$ is the number of clusters. Thus, in the case of a normal splay state the phase difference is $\pi / 2$. Furthermore, there exists a reverse compressed splay state as shown in Fig. 6.17(b). Another secondary state is the open 2-cluster state displayed in Fig. 6.17(c). For this state, the phase differences $\varphi_{0}-\varphi_{2}$ and $\varphi_{1}-\varphi_{3}$ are equal, but the phase differences $\varphi_{3}-\varphi_{0}$ and $\varphi_{2}-\varphi_{1}$ are non-equal. Figure 6.17(d) shows a compressed 2-cluster state, when $\varphi_{0}=\varphi_{2}$ and $\varphi_{1}=\varphi_{3}$, but the phase difference between the two clusters is no longer equal to $\pi$ as it would be the case in a normal 2-cluster state. This state can be found for the $\tau$ range depicted in Fig. 6.16 but with a different starting configuration (not shown here).

\subsubsection{Extended theory}

The experiments shown in the previous section for the relaxation oscillators motivate an extension of the theory of Choe et al. [2010] which was discussed in Sec. 5.2. The extended theory includes the interaction functions with a high order of Fourier coefficients and takes into account the existence of the secondary states. The secondary states can be obtained from Eq. (6.17) with the Fourier coefficients given in Tab. 6.1, if we assume constant but non-equal radii and phase differences:

$$
\begin{aligned}
r_{j} & =\text { const. } \equiv r_{0, j}, \\
\varphi_{j} & =\Omega t+\Delta \varphi_{j},
\end{aligned}
$$

with $\Delta \varphi_{j} \in \mathbb{R}$. Note that we use here a slightly different notation than in Sec. 5.2 and Subsec. 6.2.2 by omitting the index $m$ labeling the cluster type in the previous Sec. 5.2. We do this for reasons of clarity because we now need the additional index $j$ to take into account that the nodes might have different radii or frequencies.

With the ansatz Eq. (6.18), Eq. (6.16) yields the following conditions for the solutions

$$
\begin{aligned}
0 & =\left(\lambda-r_{0, j}^{2}\right) r_{0, j}+K r_{0, j+1} c_{r}^{j}, \\
\Omega & =\omega+K \frac{r_{0, j+1}}{r_{0, j}} c_{\varphi}^{j},
\end{aligned}
$$


where we used the abbreviation $(i=r, \varphi)$

$$
c_{i}^{j} \equiv{ }_{l=1}^{L} a_{l, i} \cos l\left(\Delta \varphi_{j+1}-\Delta \varphi_{j}-\Omega \tau\right)+b_{l, i} \sin l\left(\Delta \varphi_{j+1}-\Delta \varphi_{j}-\Omega \tau\right) .
$$

Equation (6.19) is an 8-dimensional ( $\Delta \varphi_{0}=0$ without loss of generality) system of transcendental equations which can be solved numerically. A variational equations can be derived using the ansatz $r_{j}(t)=r_{0, j}\left[1+\delta r_{j}(t)\right], \varphi_{j}(t)=\Omega t+\Delta \varphi_{j}$. Substituting this ansatz into Eq. (6.16) yields for the linear order of $\boldsymbol{\xi}_{j} \equiv\left(\delta r_{j}, \delta \varphi_{j}\right)$

$$
\dot{\boldsymbol{\xi}}_{j}=\mathbf{J}_{j} \boldsymbol{\xi}_{j}+\mathbf{R}_{j} \xi_{j+1}(t-\tau),
$$

with the matrices

$$
\mathbf{J}_{j}=\begin{array}{cc}
\lambda-3 r_{0, j}^{2} & -K r_{0, j+1} d_{r}^{j} \\
-K \frac{r_{0, j+1}}{r_{0, j}^{2}} c_{\varphi} & -K \frac{r_{0, j+1}}{r_{0, j}} d_{\varphi}^{j}
\end{array}
$$

and

$$
\mathbf{R}_{j}=K \begin{array}{cc}
c_{r}^{j} & r_{0, j+1} d_{r}^{j} \\
c_{\varphi}^{j} / r_{0, j} & r_{0, j+1} / r_{0, j} d_{\varphi}^{j}
\end{array},
$$

using the abbreviation $(i=r, \varphi)$

$$
d_{i}^{j} \equiv{ }_{l=1}^{L}-a_{l, i} \sin l\left(\Delta \varphi_{j+1}-\Delta \varphi_{j}-\Omega \tau\right)+b_{l, i} \cos l\left(\Delta \varphi_{j+1}-\Delta \varphi_{j}-\Omega \tau\right)
$$

Because of the unequal phase differences and radii the variational equation cannot be block-diagonalized (cf. Sec. 3.2 and 5.2 ) and thus an MSF approach is infeasible. However, the Floquet exponents $\Lambda$ can be obtained from the transcendental equation

$$
\begin{array}{cccc}
\mathbf{J}_{0}-\Lambda \mathbf{I}_{2} & \mathbf{R}_{0} e^{-\Lambda \tau} & 0 & 0 \\
0 & \mathbf{J}_{1}-\Lambda \mathbf{I}_{2} & \mathbf{R}_{1} e^{-\Lambda \tau} & 0 \\
0 & 0 & \mathbf{J}_{2}-\Lambda \mathbf{I}_{2} & \mathbf{R}_{2} e^{-\Lambda \tau}
\end{array}=0 .
$$

This transcendental equation follows from Eq. (6.21) when applying the approach to 
solve linear delay differential equations discussed in the Appendix A. Solutions of Eq. (6.19) that were found to be stable $(\Lambda<0)$ are plotted as lines in Fig. 6.16(b). As expected the lines perfectly agree with the points obtained by the analysis using DDE-BIFTOOL. The disadvantage of this analytic method compared to using the continuation software is that it is difficult to find all solutions of Eq. (6.19); i.e., in Figs. 6.16(b) the secondary states were not found analytically but only with the help of DDE-BIFTOOL. However, the analytic method gives further insight into the system, making analysis easier.

\subsection{CONCLUSION}

In this Chapter, I have discussed zero-lag synchronization and cluster synchronization in the presence of heterogeneities. In the first Section, the heterogeneities have affected the delay times, while in the second Section an experimental setup with chemical oscillators has been investigated, in which the nodes are slightly non-identical. Heterogeneities are typical for experimental situations and engineering applications. Thus, the aim of this Chapter has been to test and extend the applicability of the theory developed for identical nodes and delay times in Chapters 4 and 5 to more realistic situations characterized by a certain degree of heterogeneity.

Section 6.1 has explored heterogeneous delays in a network of delay-coupled FitzHughNagumo systems with a focus on the interplay between the delay heterogeneity and the network topology, where regular, small-world and random networks have been considered.

First, a Gaussian delay distribution has been investigated. As anticipated, the dynamics resemble closely the dynamics of a systems with a discrete delay time if the standard deviation of the delay distribution is sufficiently small. Thus, such networks can be described in good approximation by a discrete delay time equal to the mean of the distribution which allows for further analysis, for example, using the master stability function (MSF). By this means, it has been shown in Chapter 4 that a network of FitzHughNagumo systems with one discrete coupling delay stably synchronizes in the presence of excitatory coupling and desynchronizes if a critical number of inhibitory links is added.

For intermediate standard deviations, states might arise where the network still fires but with reduced synchronicity meaning that the Kuramoto order parameter drops below the value of 0.99 which we defined as the threshold for highly synchronized networks. Dynamical scenarios with reduced synchrony include traveling disruption patterns, subnetwork synchronization, or partial amplitude death. The existence of these states depends on the topology; they require a certain degree of regularity found in regular rings 
and to a lesser extent also in small-world networks. In contrast, in random networks, i.e., in the absence of any regularity, such dynamics is hardly found. If the width of the delay distribution becomes too large, global amplitude death is induced.

Global amplitude death has first been observed by Ramirez-Pastor et al. [1998] in delaycoupled Stuart-Landau oscillators, though the mechanism there is quite different from the one discussed here. Ramirez-Pastor et al. showed that an appropriate choice of the delay time changes the stability of the fixed point and yields global amplitude death. In contrast, FitzHugh-Nagumo is an excitable system. Thus, delay in the coupling is needed to generate self-sustained oscillation with a period close to the delay time [Panchuk et al., 2013]. However, as we show, if the delay distribution becomes too broad the delay cannot play this constructive role any longer.

The effect of heterogeneities in complex network has been previously studied by other authors. Atay [2003b] showed that partial amplitude death can be invoked by node heterogeneities in two coupled weakly nonlinear oscillators. Masoller and Atay [2011] studied Gaussian and exponentially distributed delays in networks of logistic maps. They also found synchronous oscillating dynamics for narrow delay distributions and amplitude death for wider distributions. However, the network topology plays a different role; for logistic maps, subnetwork synchronization is possible in random networks, while we see this dynamical behavior only in regular and (rarely) in small-world networks.

Furthermore, two discrete delay times and bimodal delay distributions have been discussed in Sec. 6.1. In the case of two discrete delay times resonance effects arise, similar to the effects observed in small network motifs [Schöll et al., 2009; Panchuk et al., 2013]. In resonance, the network spikes coherently with an interspike interval which is described by a simple linear relation.

Bimodal distributions are characterized by two dominant mean delay times, but with a distribution of the delays around these two peaks with some widths. They combine the features of the above discussed case of a distribution centered at a mean delay and the presence of two discrete delay time and, thus, yield similar or a combination of the already described effects. If the widths around the two mean delays are small, the network behaves as in the case of two discrete delay times and resonance effects play a major role. This is in analogy to the behavior we found in the case of the Gaussian distribution with narrow width where the dynamics closely resembled the ones of a network with a discrete delay time. If the widths of the bimodial distributions increases, we see dynamical scenarios already present in the case of unimodal distributions with intermediate width. In particular, in regular networks and small-world networks we see that several subnetworks coexist which spike with different interspike intervals. For 
large distribution widths, amplitude death is the only dynamical state which we have found.

In conclusion, networks with narrow unimodial or bimodial delay distributions can be well described by discrete delays, but as the width of the distribution increases, topological features have to be taken into account as they can give rise to more complex dynamics. In networks with broad distributions, spiking is not possible any longer and initial excitations die out fast, leading to global amplitude death. This behavior is similar to the case of only two coupled oscillators with distributed delay in the coupling, where the regime of amplitude death increases with the width of the delay kernel [Atay, 2003a; Kyrychko et al., 2011, 2013]. Large regular networks and to some extent small-world networks are less prone to undergo global amplitude death, since they allow more easily for stable subnetwork spiking or travelling disruptions.

Section 6.2 has investigated cluster synchronization in a ring network of chemical oscillators. Depending upon the applied voltage two different operating regimes can be distinguished: For low voltages the oscillations are smooth, while for higher voltages the oscillations are of relaxational type. In the smooth regime, the common frequency is well described by the theory of Choe et al. [2010]. This is despite the fact that Choe et al. developed their theory for identical nodes, while the oscillators in the experiment are characterized by slightly different frequencies. For higher voltages and relaxational dynamics, secondary states which are characterized by non-equal phase lags between the different clusters are observed. To capture these states we have extended the theory of Choe et al. by applying a coupling scheme which takes higher harmonics into account. In this way, we have been able to approximate the experimental interaction functions and include them into the theoretical model. This yields numerical and analytical results that agree qualitatively and quantitatively well with the experimental measurements in a parameter regime where the standard Stuart-Landau model is no longer an appropriate model.

In summary, this Chapter has investigated zero-lag and cluster synchronization in the presences heterogeneities in the delay times or nodes as commonly appearing in experimental situations or applications. In both cases, it has showed that for sufficiently small heterogeneities the dynamics are well predicted by the theory introduced in Chapters 4 and 5, which demonstrates the applicability of this theory in real-world situations. Furthermore, the results of Sec. 6.2.4 show that the theory can be extended, if necessary, to fit the more complicated situation met in an experiment. 
Part II

\section{Adaptive Control of SYNCHRONIZATION}





\section{7 | ADAPTIVE CONTROL}

In the first part of thesis, I have discussed the stability of zero-lag and cluster synchronization in complex networks. The result of this stability analysis can be used to control the coupled systems by choosing appropriate parameters. In this way, stabilizing and selecting desired states is possible. However, under certain conditions this strategy has several drawbacks:

- If there are model uncertainties, i.e., the system's governing equations are not fully known, a linear stability analysis is very difficult, if not impossible, so that appropriate parameters cannot be determined.

- In networks, we often have to deal with a "zoo" of parameters: In addition to the system and coupling parameters, topological parameters as, for example, the probability of two nodes being connected in a random network, have a great influence. Finding a set of appropriate parameters to realize a certain target state is very challenging under such conditions.

- In large networks, we often have to deal with multistability meaning that even if we find an appropriate parameter set to stabilize our desired state we still might end up in another state which is stable for the same parameter set.

- In experiments, parameters often drift, meaning that the parameters have to be readjusted from time to time.

This leads us to adaptive control which is able to address all these issues and, in particular, the last one. In an adaptively controlled system, parameters are constantly tuned in order to reach the desired state. In this way, appropriate parameters can be found automatically and followed if other parameters not under control drift. Under certain circumstances, the adaptive control can even work around the multistability of a system as the tuning of parameters induces additional degrees of freedom, as will be shown in Chapter 10. By this means the stability of all states might change and in the best case the 
multistability can be lifted in favor of the target states. However, this is not always the case: In Chapter 9, it will be discussed that the adaptive control of just one parameter fails in large rings since it cannot deal with the increasing multistability of such networks.

In this part, I will first discuss the adaptive control of generic uncoupled systems like the unstable focus (see Chapter 8), before considering ring motifs and larger networks; see Chapters 8 and 9, respectively. To design the adaptive control algorithms, I use the speedgradient (SG) method [Fradkov, 1979, 2007], for which I will give a brief recapitulation in the next Section.

There are several other approaches to adaptive control of nonlinear systems in the control literature [Krstic et al., 1995; Fradkov et al., 1999; Astolfi et al., 2008; Selivanov et al., 2015]. For the work presented in the following Chapters, we have chosen the speedgradient method because it is simple and robust. In particular, in the form used in this thesis, no knowledge about the system parameters and the local system is needed. Here, "local system" refers to the system without control in the case of uncoupled systems discussed in Chapter 8 or to one node without the coupling term in the case of the networks discussed in Chapters 9 and 10. Instead, it is sufficient to know the form of the control or coupling term as will be shown in Sec. 7.1. In Chapters 8-9 it will be demonstrated that the SG method is able to deal with drifting parameters, noise, different initial conditions, and heterogeneities of the nodes in a network.

\subsection{SPEED-GRADIENT METHOD}

Here, I review the SG method [Fradkov, 1979; Fradkov et al., 1999; Fradkov, 2005, 2007] used in the control of non-linear systems of the following form:

$$
\dot{\mathbf{z}}=\mathbf{F}(\mathbf{z}, \mathbf{u}, t) .
$$

$\mathbf{z} \in \mathbb{C}^{n}$ is the state vector, $\mathbf{u} \in \mathbb{C}^{m}$ an input (or control) variable, and $\mathbf{F}$ a nonlinear function. We define a scalar goal function $Q(\mathbf{z}(t), t)$ so that

$$
\begin{array}{rll} 
& =0, & \text { if the goal is reached, } \\
& >0, & \text { otherwise. }
\end{array}
$$

The speed (or rate) at which $Q(\mathbf{z}(t), t)$ is changing along trajectories of Eq. 7.1 is given by 
$\dot{Q}=\omega(\mathbf{z}, \mathbf{u}, t)$ and can be calculated as follows:

$$
\omega(\mathbf{z}, \mathbf{u}, t)=\frac{\partial Q(\mathbf{z}, t)}{\partial t}+\left[\nabla_{\mathbf{z}} Q(\mathbf{z}, t)\right]^{\mathrm{T}} \mathbf{F}(\mathbf{z}, \mathbf{u}, t) .
$$

Then, the SG method changes the control vector $\mathbf{u}$ in the direction of the negative gradient of $\omega(\mathbf{z}, \mathbf{u}, t)$ with respect to input variables:

$$
\frac{d \mathbf{u}}{d t}=-\gamma \nabla_{\mathbf{u}} \omega(\mathbf{z}, \mathbf{u}, t)
$$

where $\gamma=\gamma^{\mathrm{T}}>0$ is a positive definite gain matrix.

The idea of this algorithm is the following: The term $-\nabla_{u} \omega(\mathbf{z}, \mathbf{u}, t)$ points to the direction in which the value of $\dot{Q}$ decreases with the highest speed. Therefore, if one forces the control signal to "follow" this direction, the value of $\dot{Q}$ will decrease and finally be negative. As soon as $\dot{Q}<0$, the goal function $Q$ will decrease and eventually tend to zero.

Note that I assumed that $Q(\mathbf{z}(t), t)$ is independent of $\mathbf{u}$. The reason is that a goal function which only relies on the state of the system is straight forward to construct, while a goal function which also depends on the control variables requires an a priori knowledge of the values of these variables that lead to successful control. However, if these values were known, there would be no need to apply an adaptive control algorithm. The main advantage of the SG method compared to standard gradient methods is that it can be used for such goal functions because the speed $\omega(\mathbf{z}, \mathbf{u}, t)$ depends on $\mathbf{u}$ even though the goal function $Q(\mathbf{z}(t), t)$ does not.

Depending on the control problem, the choice of appropriate values for the entries of $\gamma$ might be decisive. If the entries are small, the speed of convergence to the goal state decreases. On the other hand, if their values become too big, undesirable oscillations might appear. However, this is only a tendency. In some of the problems considered in the next Chapter, the control is successful for a wide range of values of the entries of $\gamma$.

We now assume that $\mathbf{F}(\mathbf{z}, \mathbf{u}, t)$ in Eq. (7.1) consists of one part that depends on $\mathbf{u}$ and one that does not, i.e., $\mathbf{F}(\mathbf{z}, \mathbf{u}, t)=\mathbf{F}_{1}(\mathbf{z}, t)+\mathbf{F}_{2}(\mathbf{z}, \mathbf{u}, t)$. This allows us to rewrite Eq. (7.4) as

$$
\frac{d \mathbf{u}}{d t}=-\gamma \nabla_{\mathbf{u}} \omega(\mathbf{z}, \mathbf{u}, t)=-\gamma \nabla_{\mathbf{u}}\left[\nabla_{\mathbf{z}} Q(\mathbf{z}, t)\right]^{\mathrm{T}} \nabla_{\mathbf{u}} \mathbf{F}_{2}(\mathbf{z}, \mathbf{u}, t)
$$


meaning that we do not have to know the full system equations but only the part dependent on $\mathbf{u}$. This is particularly interesting for networks, where we often want to control the coupling parameters, see Chapter 9, or the topology, see Chapter 10. In this case it is sufficient to know the coupling term but one does not have know the local dynamics of the nodes. The same applies to the adaptive form of time delayed feedback control (TDFC), which is introduced in Chapter 8.

There exist different versions of analytic conditions guaranteeing that the control goal $Q(\mathbf{z}(t), t)$ can be achieved in the system (7.1) with adaptation law (7.4); see [Fradkov, 1979; Shiriaev and Fradkov, 2000]. The main condition is that a constant value of the parameter $\mathbf{u}^{*}$ has to exist ensuring attainability of the goal in the system

$$
\dot{\mathbf{z}}=\mathbf{F}\left(\mathbf{z}, \mathbf{u}^{*}, t\right) .
$$

Details can be found in the control related literature [Fradkov, 1979; Shiriaev and Fradkov, 2000].

\subsection{SUMMARY}

In situations where parameters drift or are unknown, adaptive control methods can be used to automatically find appropriate parameters. Furthermore, they can be applied in situations where it is necessary to deal with a variety of different parameters such that a systematic consideration of the system's parameter dependency is impossible. Then, the adaptation can be used to find a parameter set which realizes a desired control goal.

A particularly simple and easy to apply control method is the speed gradient (SG) method. To apply the SG method, a goal function is constructed which has a minimum in the target state. The SG method minimizes the speed at which the goal function changes along trajectories of the system. If the goal function is appropriately chosen, its speed will become negative at some point in time, and, thus the goal function decreases until the goal is reached. 


\section{8 | AdAPTIVE TIME-DELAYED FEEDBACK CON- TROL}

The previous Chapter discussed the speed-gradient (SG) method as an easy and straightforwardly applicable scheme for deriving adaptive controllers. Before applying the SG method in the control of networks, I discuss in this Chapter its application to uncoupled systems as a proof of principle of its wide applicability in the control of systems with time delay and to discuss its strengths and weaknesses. In particular, I show that the SG method can be used to turn time-delayed feedback control (TDFC) and extended time-delayed feedback control (ETDFC) into an adaptive control scheme by tuning of the coupling strength. This scheme was developed in [Guzenko et al., 2008; Lehnert et al., 2011b].

This Chapter is structured as follows: Section 8.1 is a recapitulation on standard TDFC where standard will mean in the following without adaptation of parameters. In Secs. 8.2 and 8.3 an adaptive controller based on the TDFC is developed and applied in the stabilization of an unstable fixed point and an unstable periodic orbit embedded in a chaotic attractor. Furthermore, the influences of noise and parameter drifts are discussed. Section 8.4 is a conclusion summarizing the results and comparing them to existing adaptive control schemes.

\subsection{TIME-DELAYED FEEDBACK CONTROL}

Since the seminal paper of Ott et al. [1990], chaos control is a central topic in the theory of non-linear systems. A particular simple but efficient scheme to control chaos, i.e., to stabilize unstable periodic orbits embedded in a strange attractor, was suggested by Pyragas [1992], and is known as time-delayed feedback control (TDFC). This method generates a feedback signal from the difference of the current state of the system to its 
counterpart some time units $\tau$ in the past. TDFC has also been applied in the control of unstable steady states [Ahlborn and Parlitz, 2004; Rosenblum and Pikovsky, 2004; Hövel and Schöll, 2005].

The TDFC combines several advantages. For appropriate values of $\tau$ it is noninvasive, meaning that the orbit or fixed point under control is stabilized but not changed. Furthermore, the control scheme does not rely on a reference system or on an a priori knowledge of the system itself. In its most simple version, it has only a small number of control parameters, i.e., the feedback gain $K$ and time delay $\tau$. For reviews on TDFC see [Schöll and Schuster, 2008; Grebogi, 2010].

A generalization of the original Pyragas scheme, suggested by Socolar et al. [1994], uses multiple time delays. This extended time-delayed feedback control (ETDFC) introduces a memory parameter $R$, which serves as a weight of states further in the past. Dahms et al. [2007] showed that this method is able to control unstable fixed points for a larger range of parameters compared to the original TDFC scheme. For reviews on TDFC and ETDFC see [Schöll and Schuster, 2008; Grebogi, 2010].

The choice of appropriate control parameter is crucial for the success of TDFC as has been shown in [Hövel and Schöll, 2005; Dahms et al., 2007]. Adaptive methods can be used to find these parameters and thus widen the applicability of TDFC which is the aim of the following Sections.

\subsection{Stabilization OF AN UNSTABlE FIXED POINT}

First, we will consider the stabilization of an unstable focus which is a generic model as it arises in the linearization of a system with an unstable fixed point close to a subcritical Hopf bifurcation. The uncontrolled unstable fixed point is given by

$$
\begin{aligned}
\dot{x} & =\lambda x+\omega y, \\
\dot{y} & =-\omega x+\lambda y,
\end{aligned}
$$

where $\lambda$ and $\omega$ are positive real numbers. $\lambda$ may be viewed as the bifurcation parameter governing the distance from the instability threshold, i.e., from the Hopf bifurcation, and $\omega$ is the intrinsic eigenfrequency of the focus. As Eq. (8.1) is already linear, its Jacobian A 
can simply be determined as

$$
\mathbf{A}=\begin{array}{cc}
\lambda & \omega \\
-\omega & \lambda
\end{array} .
$$

The eigenvalues $\Lambda_{0}$ of $\mathbf{A}$ are given by $\Lambda_{0}=\lambda \pm \mathrm{i} \omega$ such that for $\lambda>0$ and $\omega \neq 0$ the fixed point is an unstable focus. Applying TDFC in order to stabilize this fixed point, Eq. (8.1) reads:

$$
\begin{aligned}
& \dot{x}(t)=\lambda x(t)+\omega y(t)-K[x(t)-x(t-\tau)], \\
& \dot{y}(t)=-\omega x(t)+\lambda y(t)-K[y(t)-y(t-\tau)],
\end{aligned}
$$

where the feedback gain $K$ and the time delay $\tau$ are real numbers. Note that the feedback term vanishes if the fixed point $\left(x^{*}, y^{*}\right)$ is stabilized since $x^{*}(t-\tau)=x^{*}(t)$ and $y^{*}(t-\tau)=$ $y^{*}(t)$ for all $t$, indicating the noninvasiveness of the TDFC method.

Since the control force applied to the $i$ th component of the system involves only the same component, this control scheme is called diagonal coupling [Beck et al., 2002] and is suitable for an analytical treatment. Hövel and Schöll [2005] showed that in the $(K, \tau)-$ plane tongues exist for which the fixed point can be stabilized, i.e., for a given $\tau$ there is a $K$-interval for which the control is successful. In this interval the real part $\operatorname{Re} \Lambda$ of the eigenvalue of the focus with standard TDFC is negative; in Fig. 8.1 showing $\operatorname{Re} \Lambda$ as a function of $K$ and $\tau$ negative $\operatorname{Re} \Lambda$ corresponds to the blue region. In particular, the $K$-interval for successful control is largest for odd multiples of half of the intrinsic period $T_{0} \equiv 2 \pi / \omega$, i.e., $\tau=(2 n+1) T_{0} / 2, n \in \mathbb{N}$, while for even multipliers, i.e., $\tau=n T_{0}, n \in \mathbb{N}$, the control fails for all values of $K$. In the following, we assume that the value of $\tau$ is known and appropriately chosen. The value of $K$ will initially be zero; the adaptive controller will tune $K$ such that its final value is in the $K$-interval of successful control for the given $\tau$ value.

To obtain an adaptation algorithm for the feedback gain $K$ according to the standard procedure of the speed-gradient method, the following the goal function or goal function is chosen

$$
Q(x, y, t)=\frac{1}{2}[x(t)-x(t-\tau)]^{2}+[y(t)-y(t-\tau)]^{2} .
$$

Successful control yields $Q(x, y, t) \rightarrow 0$ as $t \rightarrow \infty$. The speed-gradient algorithm (7.4) with $\mathbf{u}=K$ is given by $\dot{K}=-\gamma \nabla_{K} \omega(x, y, K, t)$, where $\nabla_{K}$ denotes $\partial / \partial K$ and $\omega(x, y, K, t)=\dot{Q}$ 


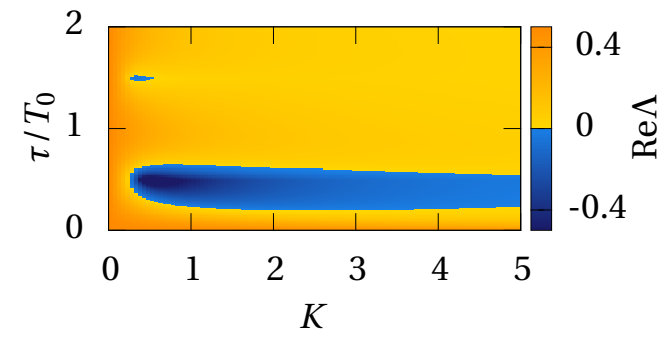

Figure 8.1.: Real part $\operatorname{Re} \Lambda$ of the eigenvalue of the unstable focus with standard TDFC vs. $K$ and $\tau . \quad \lambda=0.5$, $\omega=\pi$. Figure modified from [Hövel, 2009].

is the rate of change of the goal function. For the above goal function (8.4), we obtain:

$$
\dot{Q}=[x(t)-x(t-\tau)][\dot{x}(t)-\dot{x}(t-\tau)]+[y(t)-y(t-\tau))][\dot{y}(t)-\dot{y}(t-\tau)] .
$$

The time derivatives of $x$ and $y$ are given by Eq. (8.3). Thus, the speed-gradient method leads to the following equation for the feedback gain:

$$
\begin{aligned}
\dot{K}(t)= & \gamma\{[x(t)-x(t-\tau)][x(t)-2 x(t-\tau)+x(t-2 \tau)] \\
& +[y(t)-y(t-\tau)][y(t)-2 y(t-\tau)+y(t-2 \tau)]\} .
\end{aligned}
$$

Owing to homogeneity the right hand sides of Eqs. (8.3) and (8.6) and without loss of generality the adaptation gain $\gamma$ can be chosen as 1 , because Eqs. (8.3) and (8.6) can be rescaled by the transformation

$$
\begin{aligned}
& x(t) \longrightarrow x(t) / \sqrt{\gamma}, \\
& y(t) \longrightarrow y(t) / \sqrt{\gamma} .
\end{aligned}
$$

Figure 8.2 depicts the time series of $x$ and $K$ according to Eqs. (8.3) and (8.6) for different initial conditions $x(0) \in[0.02,0.5]$ in steps of 0.02 from light green to dark blue and $y(0)=$ 0 . In all simulations $x(t)=y(t)=0$ for $t<0$ and $K(t)=0$ for $t \leq 2 \tau$. Figure 8.2(a) shows that the adaptation algorithm works for a large range of initial conditions. Naturally, for initial conditions close to the fixed point the goal is reached faster. If the system starts initially too far from the fixed point $(x(0)>0.85, y(0)=0)$ the control fails (curves not shown). Note, however, that the basin of attraction can be enlarged by increasing $\gamma$. In fact, due to the scaling, invariance (8.7), the maximum value of $|x(0)|$ that still leads 

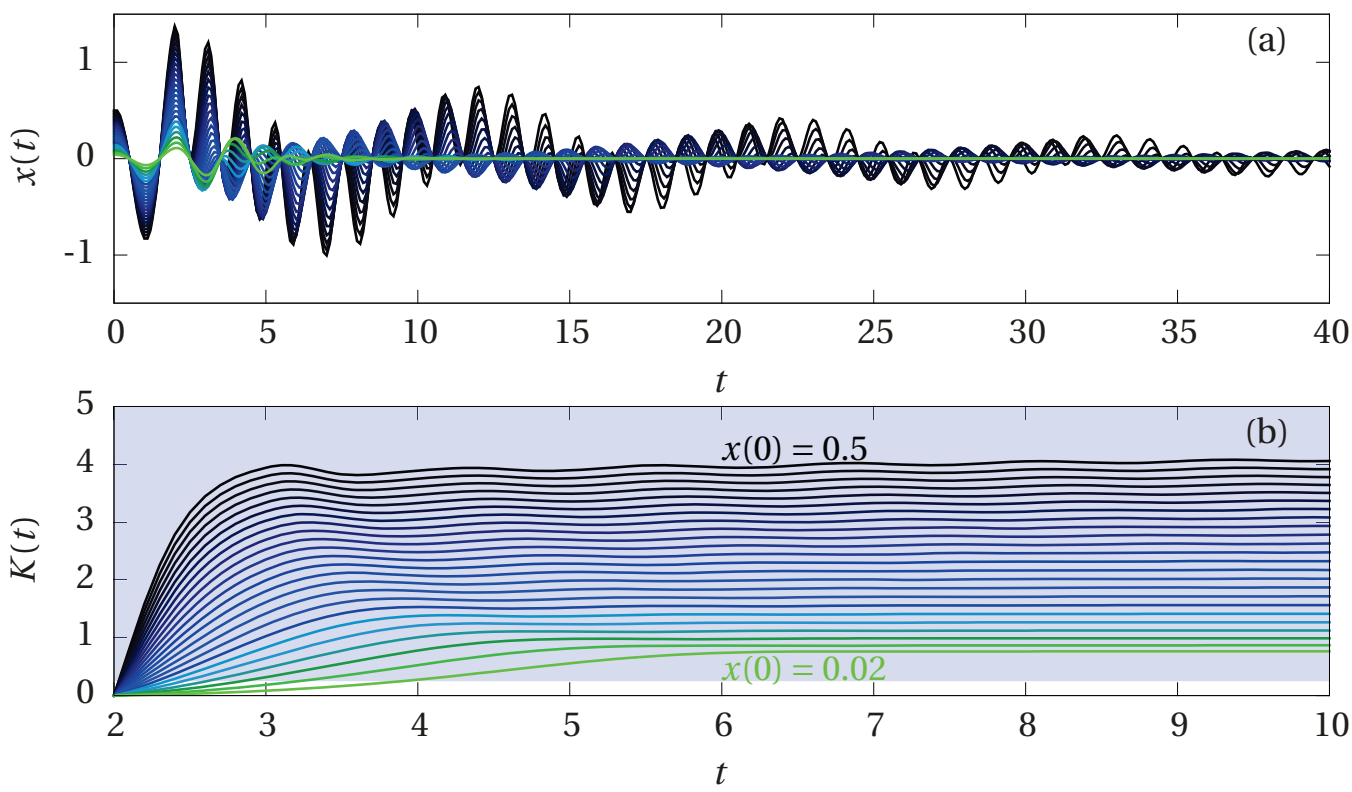

Figure 8.2.: Adaptive control of the fixed point: (a) Time series $x(t)$ and (b) feedback gain $K(t)$ for different initial conditions: $x(0) \in[0.02,0.5]$ in steps of 0.02 (from light green to dark blue; in panel (b) from top to bottom), $y(0)=0$. Blue shaded region: $K$ interval of successful control for TDFC. Parameters: $\lambda=0.5, \omega=\pi, \gamma=1, \tau=1$. Figure from [Lehnert et al., 2011b].

to successful control is proportional to $\sqrt{\gamma}$. The $K$-interval for which standard TDFC is successful (as discussed above and analytically calculated in [Hövel and Schöll, 2005]) is given by the blue shaded area. As can be seen in Fig. 8.2(b), the adaptive algorithm converges to some appropriate value of $K$ in this interval depending upon the initial conditions.

So far the value of $\tau$ optimal for TDFC and linked to the largest $K$-interval was studied, i.e., $\tau=T_{0} / 2$. However, as Fig. 8.3 demonstrates the adaptive algorithm works for a range of $\tau$, i.e., for any value of $\tau$ within the domain of stability of the TDFC control [Hövel and Schöll, 2005]. Black circles depict the transient time $t_{c}$ after which the control goal is reached in dependence on the time delay $\tau$. We consider the goal to be reached if the goal function $Q$ becomes sufficiently small. The transient time is defined by the bound $\langle Q\rangle \equiv \int_{t_{c}-2 \tau}^{t_{c}} Q\left(t^{\prime}\right) d t^{\prime}\left\langle 2 \tau \times 10^{-10}\right.$. The dark purple shaded regions correspond to the analytically obtained $\tau$-intervals of TDFC control [Hövel and Schöll, 2005]. Inside these intervals, $t_{c}$ has a finite value confirming that the adaptive control scheme 
adjusts the feedback gain $K$ to an appropriate value. For a comparison with the transient time of TDFC see [Hinz et al., 2011] where a power law scaling $t_{c} \sim\left(K-K_{c}\right)^{-1}$ with respect to the fixed feedback gain $K$ has been found (here $K_{c}$ corresponds to the boundaries of stability). The curves corresponding to non-zero memory parameter $R$ (crosses and squares) will be discussed below where the speed-gradient method is applied to the ETDFC scheme.

Mathematically speaking, the goal of the control method is to change the sign of the real part of the eigenvalue, leading to a decay of perturbations from the target fixed point. For a thorough analysis of the stability of the fixed point, a linear stability analysis for the system Eqs. (8.3) and (8.6) is performed. This system has the fixed point $\left(0,0, K^{*}\right)$ for any $K^{*}=$ const . Linearization of Eqs. (8.3) and (8.6) around the fixed point yields

$$
\begin{aligned}
\delta \dot{x}(t) & =\lambda \delta x(t)+\omega \delta y(t)-K[x(t)-x(t-\tau)], \\
\delta \dot{y}(t) & =-\omega \delta x(t)+\lambda \delta y(t)-K[\delta y(t)-\delta y(t-\tau)], \\
\delta \dot{K}(t) & =0 .
\end{aligned}
$$

Equation (8.8) is a linear delay differential equation and can be solved by considering the roots of the corresponding transcendental eigenvalue equation as is discussed in Appendix A. The transcendental equation is obtained by inserting the ansatz $\delta x, \delta y, \delta K \propto$ $\exp (\Lambda t)$ into Eq. (8.8):

$$
\begin{aligned}
& \lambda-K\left(1-e^{-\Lambda \tau}\right)-\Lambda \quad \omega \quad 0 \\
& 0=\operatorname{det} \quad-\omega \quad \lambda-K\left(1-e^{-\Lambda \tau}\right)-\Lambda \quad 0 \\
& \begin{array}{lll}
0 & 0 & -\Lambda
\end{array} \\
& =-\Lambda\left[\lambda+\mathrm{i} \omega-K\left(1-e^{-\Lambda \tau}\right)-\Lambda\right]\left[\lambda-\mathrm{i} \omega-K\left(1-e^{-\Lambda \tau}\right)-\Lambda\right],
\end{aligned}
$$

which can be solved numerically. Equation (8.9) equation is equal to the case of TDFC control with constant feedback gain considered in [Hövel and Schöll, 2005] except for the factor $\Lambda$. In other words, the adaptively controlled system has an additional eigenvalue at $\Lambda=0$. It results from the translation invariance of the system in the direction of $K$ on the fixed point line $(0,0, K)$. This means that the $K$ values found in the case of the standard TDFC lead again to a stabilization of the fixed point. The advantage of an adaptive controller is that an appropriate feedback gain is realized in an automated way, i.e., without prior knowledge of the domain of stability, as long as a stability domain exists for this value of $\tau$. 


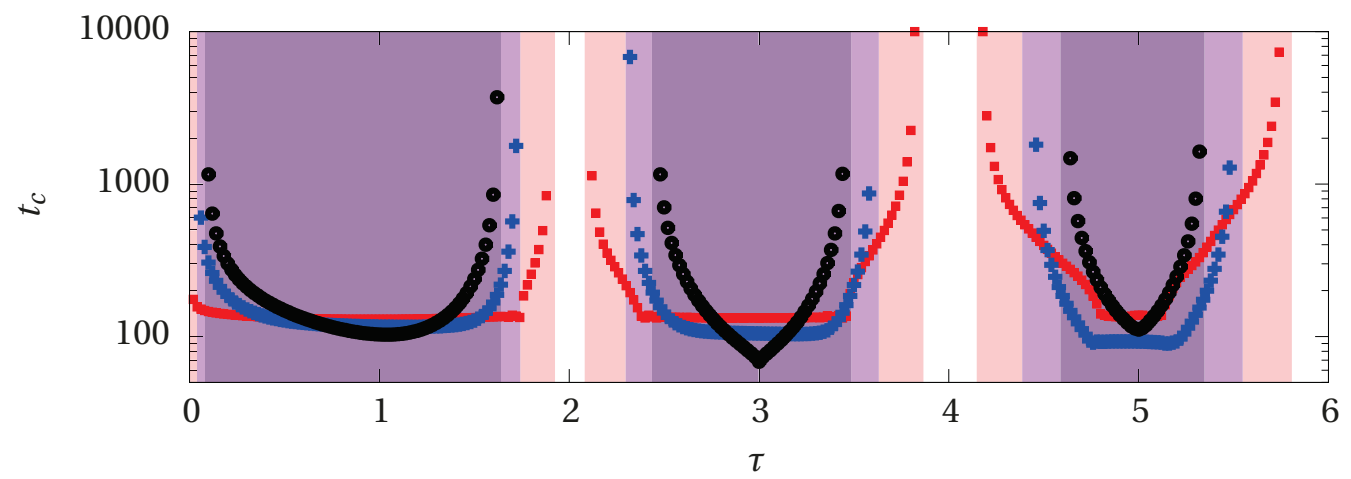

Figure 8.3.: Transient time $t_{c}$ after which the control goal is reached in dependence on the delay time $\tau$ for TDFC (black circles) and ETDFC with $R=0.35$ (blue crosses), and $R=0.95$ (red squares). The dark purple, bright purple, and red shaded regions denote the possible range of $\tau$ for $R=0,0.35$, and 0.95, respectively. Parameters as in Fig. 8.2. Figure from [Lehnert et al., 2011b].

\subsubsection{Robustness towards noise and drifting parameters}

As discussed at the beginning of Chapter 7, an additional advantage of an adaptive control scheme is that it allows one to follow slow changes of the system parameters, which are usually present in experimental situations. To test the ability of the here presented adaptive control scheme to cope with such parameter drifts, $\lambda$ is slowly varied in the following way: $\lambda(t)=0.01+1.8 \sin (0.001 t)$. The result is illustrated in Fig. 8.4. In Fig. 8.4(a) the region of stability of the standard TDFC in the $(\lambda, K)$-plane (according to [Hövel and Schöll, 2005]) is marked by blue shading. We now slowly increase $\lambda$ from its initial value 0.01 . $K$ follows the change in such a way that whenever the lower boundary of the stability region is crossed and the fixed point becomes unstable, the adaptation algorithm adjusts $K$ such that the stable region is reentered. This creates a step-like trajectory in the $(\lambda, K)$-plane, which is depicted as a red curve with an arrow. Finally, if $\lambda$ is decreased again, $K$ does not change because it already has attained a value for which the control works in a broad $\lambda$-interval resulting in a horizontal trajectory in the $(\lambda, K)$-plane. Figure 8.4(b) depicts the corresponding time series of $K(t)$ as a blue curve, and of the drifting parameter $\lambda(t)$ as a red curve, respectively.

To test the robustness of the control algorithm, Gaussian white noise $\xi_{i}(i=1,2)$ with zero mean and unity variance $\left(\left\langle\xi_{i}(t)\right\rangle=0,\left\langle\xi_{i}(t) \xi_{j}\left(t-t^{\prime}\right)\right\rangle=\delta_{i j} \delta\left(t-t^{\prime}\right)\right)$ is added to the 

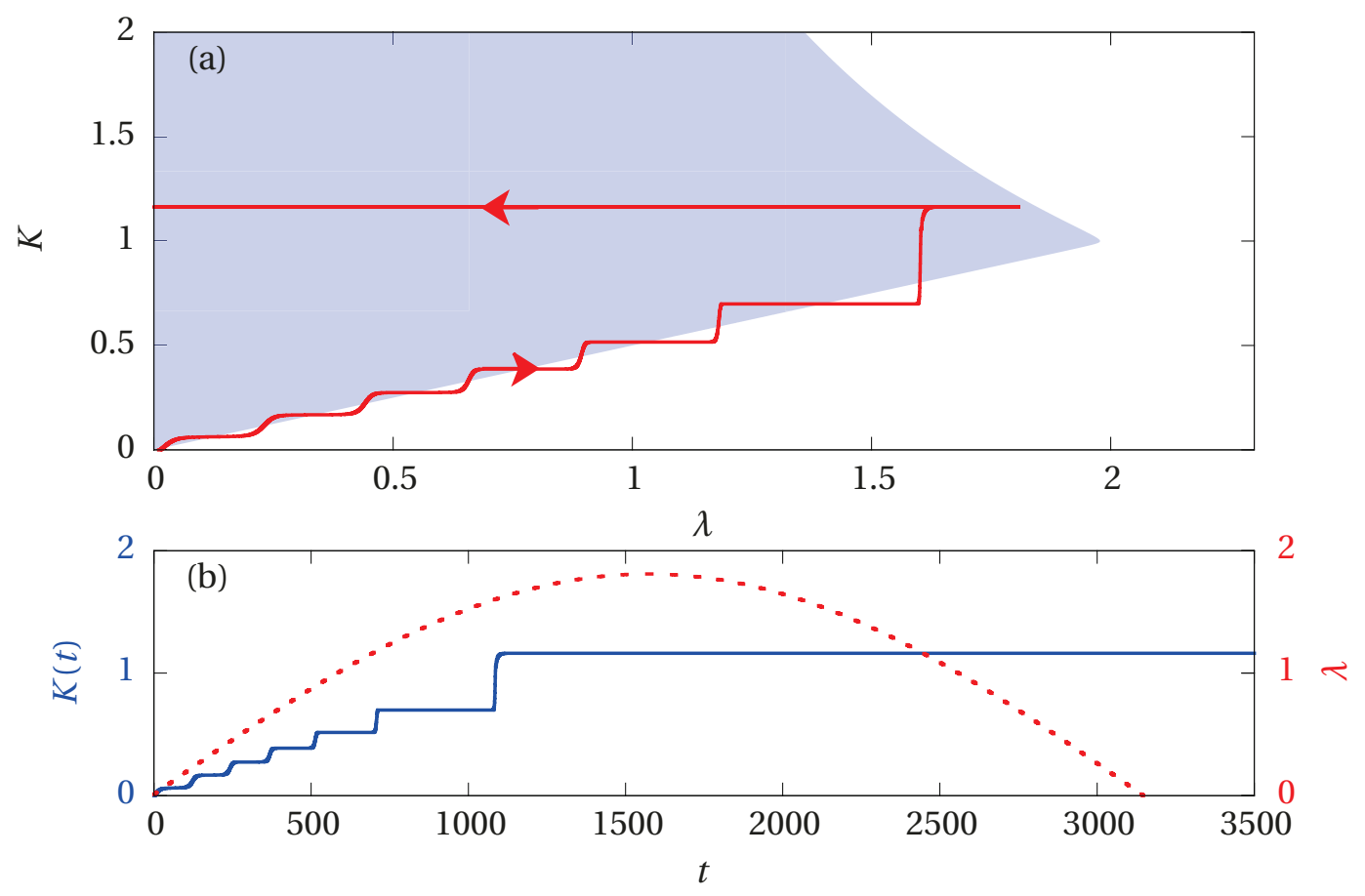

Figure 8.4.: Adaptive control of the fixed point for slowly drifting system parameter $\lambda$. (a) Adaptive adjustment of $K$ in the $(\lambda, K)$-plane. Blue shaded region: region of stability of the standard TDFC control. Red line with arrow: adaptation of feedback gain $K$ if $\lambda$ is slowly changed $(\lambda(t)=0.01+1.8 \sin (0.001 t)$ ) (b) Corresponding time series $K(t)$ (blue solid line) and $\lambda(t)$ (dashed red line). Other parameters as in Fig. 8.2. Figure from [Lehnert et al., 2011b]. 

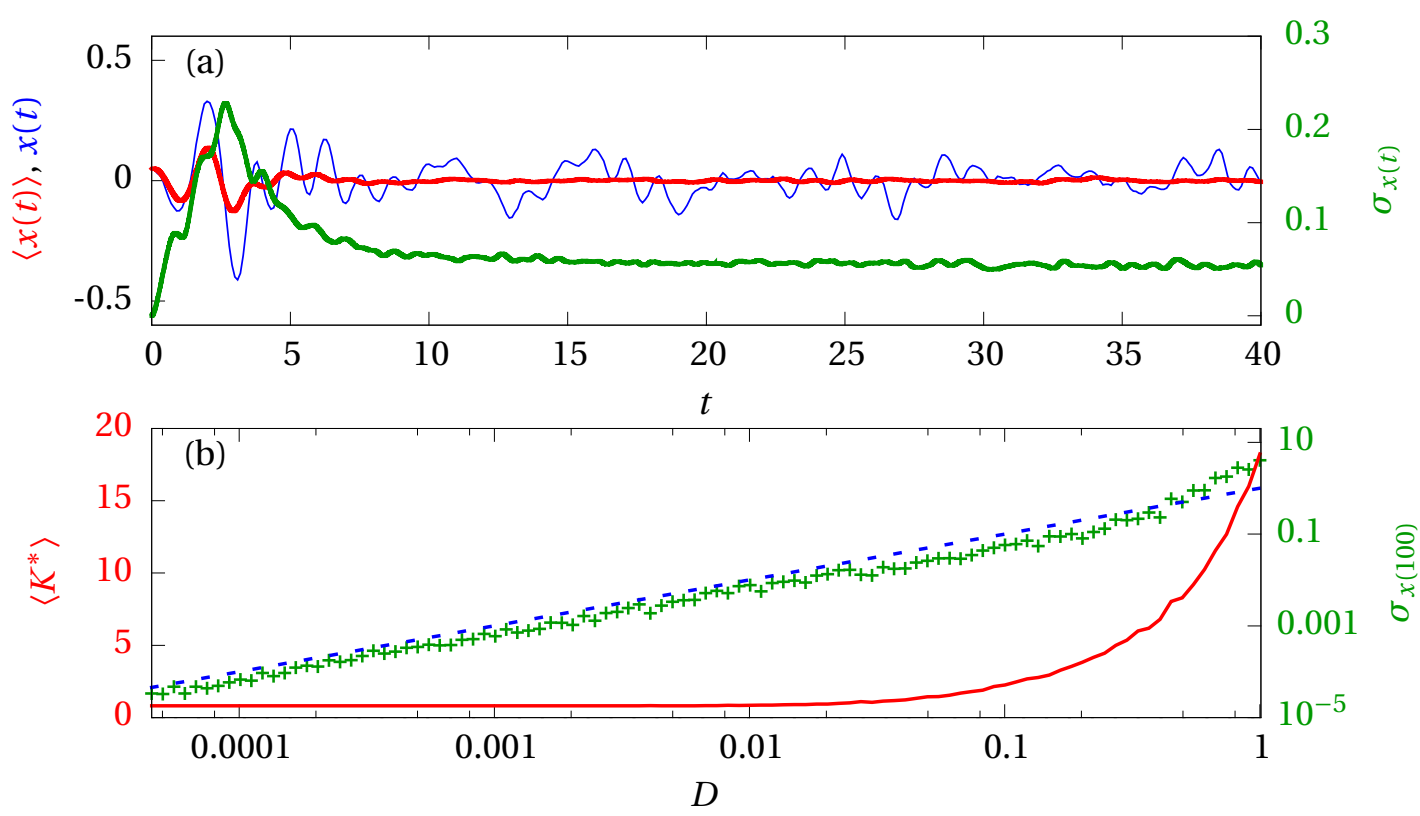

Figure 8.5.: Robustness to noise. (a): Red curve: ensemble average $\langle x(t)\rangle$ of 200 realizations; blue curve: $x(t)$ for one example trial; green curve: corresponding standard deviation $\sigma_{x(t)}$ of $x(t)$ for a fixed noise intensity $D=0.1$. (b): Green crosses: standard deviation $\sigma_{x(100)}$ of $\langle x(t=100)\rangle$; dashed blue line: standard deviation of the input noise given by $D$; red curve: asymptotic value $K^{*}$ of the feedback gain. Parameters: $\gamma=0.001, x(0)=0.05, y(0)=0$. Other parameters as in Fig.8.2. Figure from [Lehnert et al., 2011b].

system variables $x$ and $y$ :

$$
\begin{aligned}
\dot{x}(t) & =\lambda x(t)+\omega y(t)-K(t)[x(t)-x(t-\tau)]+D \xi_{1}(t), \\
\dot{y}(t) & =-\omega x(t)+\lambda y(t)-K(t)[y(t)-y(t-\tau)]+D \xi_{2}(t),
\end{aligned}
$$

where $D$ is the strength of the noise.

In Fig. 8.5(a) the ensemble average over 200 realizations, i.e., $\langle x(t)\rangle=1 / 200 \quad{ }_{i=1}^{200} x_{i}(t)$, for $D=0.1$ (intermediate noise) is depicted as a red curve. The blue curve exemplarily depicts one realization. The corresponding standard deviation $\sigma_{x(t)}$ of $x(t)$ is shown as a green curve. The control is successful in all realizations: For large $t$ the mean $\langle x(t)\rangle$ approaches the fixed point value at zero; the small fluctuations visible are due to the 
finite number of realizations. The standard deviation approaches a value smaller than the standard deviation of the input noise.

This is further elaborated in Fig. 8.5(b), which depicts the standard deviation $\sigma_{x}$ at $t=$ 100 versus the noise strength $D$ as green crosses. If $D$ becomes too large the standard deviation exceeds the one of the input noise indicated by the dashed blue line. This is the case for $D \gtrsim 0.4$. Then the control algorithm will generally fail (time series not shown here): The oscillations of $x(t)$ become larger with increasing $t$. Accordingly, the standard deviation $\sigma_{x(t)}$ increases with $t$ indicating that the dynamics is dominated by noise which forces at least some of the realizations to diverge. The red curve in Fig. 8.5(b) depicts the asymptotic value $K^{*}$ of the feedback gain. For intermediate noise strength, an increased feedback gain $K$ compensates the influence of noise ensuring that the control is still successful. For too large $D, K$ increases to a value beyond the domain of stability and stabilization cannot be achieved. In conclusion, the adaptive algorithm is quite robust to noise (the escape rate is vanishingly small for $D \lesssim 0.4$ ) and only fails for large noise $(D \gtrsim 0.4)$.

Note that the method still works if the control term is added only to the $x$-component. Then, using $Q(x)=[x(t)-x(t-\tau)]^{2} / 2$ as a goal function leads to qualitatively very similar results. This observation becomes relevant for experimental realizations of the timedelayed feedback control when only certain components of the system under control are accessible for measurements [Flunkert and Schöll, 2011].

\subsubsection{Extended time-delayed feedback control}

Next, the ETDFC scheme is considered [Socolar et al., 1994]:

$$
\dot{\mathbf{X}}(t)=\mathbf{A} \mathbf{X}(t)-\mathbf{F}(t),
$$

where $\mathbf{X}=(x, y)$ and $\mathbf{A}$ is given by Eq. (8.2). The ETDFC control force $\mathbf{F}$ can be written as

$$
\begin{aligned}
\mathbf{F}(t) & =K{ }_{n=0}^{\infty} R^{n}[\mathbf{X}(t-n \tau)-\mathbf{X}(t-(n+1) \tau)] \\
& =K \mathbf{X}(t)-(1-R)_{n=1}^{\infty} R^{n-1} \mathbf{X}(t-n \tau) \\
& =K[\mathbf{X}(t)-\mathbf{X}(t-\tau)]+R \mathbf{F}(t-\tau)
\end{aligned}
$$


Here, $R \in(-1,1)$ is a memory parameter that takes into account those states that are delayed by more than one time interval $\tau$. Note that $R=0$ recovers the TDFC control scheme introduced by Pyragas [1992]. The first form of the control force, Eq. (8.13a), indicates the noninvasiveness of the ETDFC method because $\mathbf{X}^{*}(t-\tau)=\mathbf{X}^{*}(t)$ if the fixed point is stabilized. The third form, Eq. (8.13c), is suited best for an experimental implementation since it involves states further than $\tau$ in the past only recursively.

To apply a speed-gradient adaptation algorithm for the feedback gain $K$, we follow the same strategy as before and choose the goal function as $Q(x)=\left[(x(t)-x(t-\tau))^{2}+(y(t)-\right.$ $\left.y(t-\tau))^{2}\right] / 2$. Using again $\dot{K}=-\gamma \nabla_{K} \dot{Q}$, we obtain for a diagonal control scheme

$$
\begin{aligned}
\dot{K}(t)= & \gamma\left\{[x(t)-x(t-\tau)]\left[(x(t)-2 x(t-\tau)+x(t-2 \tau))+R S_{x}(t-\tau)\right]\right. \\
& +[y(t)-y(t-\tau)]\left[(y(t)-2 y(t-\tau)+y(t-2 \tau))+R S_{y}(t-\tau)\right\}
\end{aligned}
$$

with the abbreviations

$$
\begin{aligned}
S_{x}(t) & =R_{n=0}^{\infty} R^{n}[x(t-n \tau)-2 x(t-(n+1) \tau)+x(t-(n+2) \tau)] \\
& =[x(t)-2 x(t-\tau)+x(t-2 \tau)]+R S_{x}(t-\tau), \\
S_{y}(t) & ={ }_{n=0}^{\infty}[y(t-n \tau)-2 y(t-(n+1) \tau)+y(t-(n+2) \tau)] \\
& =[y(t)-2 y(t-\tau)+y(t-2 \tau)]+R S_{y}(t-\tau) .
\end{aligned}
$$

In [Dahms et al., 2007] the domains of stability for which ETDFC works were obtained analytically. The intervals of $\tau$ increase with $R$ and are larger than in the case of TDFC $(R=0)$.

Figure 8.3 depicts the transient time $t_{c}$ in dependence on $\tau$ for $R=0.35$ and $R=0.95$ as blue crosses and red squares, respectively. The red and purple shaded regions indicate the ranges of stability of $\tau$ of the standard ETDFC [Dahms et al., 2007]. Close to the optimal values of $\tau, \tau=T_{0} / 2(2 n+1), n \in \mathbb{N}, t_{c}$ is small, demonstrating the efficiency of the adaptive algorithm. Towards the boundary of the domain of stability, $t_{c}$ increases but remains finite. The control algorithm only fails very close to the border of the intervals of $\tau$. To summarize, the adaptive control algorithm for ETDFC converges to appropriate values of $K$ and stabilizes the fixed point even for parameters where standard TDFC fails. 


\subsection{STABILIZATION OF AN UNSTABLE PERIODIC ORBIT IN THE RÖSSLER SYSTEM}

In this Section, the adaptive TDFC is utilized in the stabilization of an unstable periodic orbit of the Rössler system which is a paradigmatic model for chaotic systems. The system exhibits chaotic oscillations born via a cascade of period-doubling bifurcations and is given by the following equations including the control term:

$$
\begin{aligned}
\dot{x}(t) & =-y(t)-z(t)-K[x(t)-x(t-\tau)], \\
\dot{y}(t) & =x(t)+a y(t) \\
\dot{z}(t) & =b+z(t)[x(t)-\mu] .
\end{aligned}
$$

In the following, the parameter values are fixed as $a=0.2, b=0.2$, and $\mu=6.5$ in the chaotic regime. Unstable periodic orbits with periods $T_{1} \approx 5.91679$ ("period-1 orbit") and $T_{2} \approx 11.82814$ ("period-2 orbit") are embedded in the chaotic attractor. As shown in [Balanov et al., 2005] by a bifurcation analysis, application of TDFC with $\tau=T_{1}$ and $0.24<$ $K<2.3$ stabilizes the period-1 orbit, and it becomes the only attractor of the system. In [Just et al., 1997] it was predicted analytically by a linear expansion that control using the standard TDFC can only be realized in a finite range of the values of $K$ : At the lower control boundary the limit cycle undergoes a period-doubling bifurcation, and at the upper boundary a Hopf bifurcation occurs generating a stable or an unstable torus from a limit cycle (Neimark-Sacker bifurcation). Using the goal function

$$
Q(x)=\frac{1}{2}[x(t)-x(t-\tau)]^{2},
$$

the speed-gradient adaptation algorithm for $K$ is obtained as in the previous Section [Guzenko et al., 2008]:

$$
\dot{K}(t)=\gamma[x(t)-x(t-\tau)][x(t)-2 x(t-\tau)+x(t-2 \tau)]
$$

with the initial value $K(0)=0$. Figure 8.6(a) depicts the time series of a stabilized orbit for a time delay $\tau=T_{1}$. Panel (b) shows that the adaptation algorithm converges to an appropriate value of $K$ and the goal function tends to zero.

Contrary to the previous case, it is not possible to set the adaptation gain $\gamma$ to 1 by rescaling the system but the value of $\gamma$ is crucial for successful control. To explore the role of $\gamma$, the fraction of realizations $f_{c}$ is determined where the control goal is reached as a function of $\gamma$. The initial conditions are Gaussian distributions with the mean $\langle x(0)\rangle=$ 

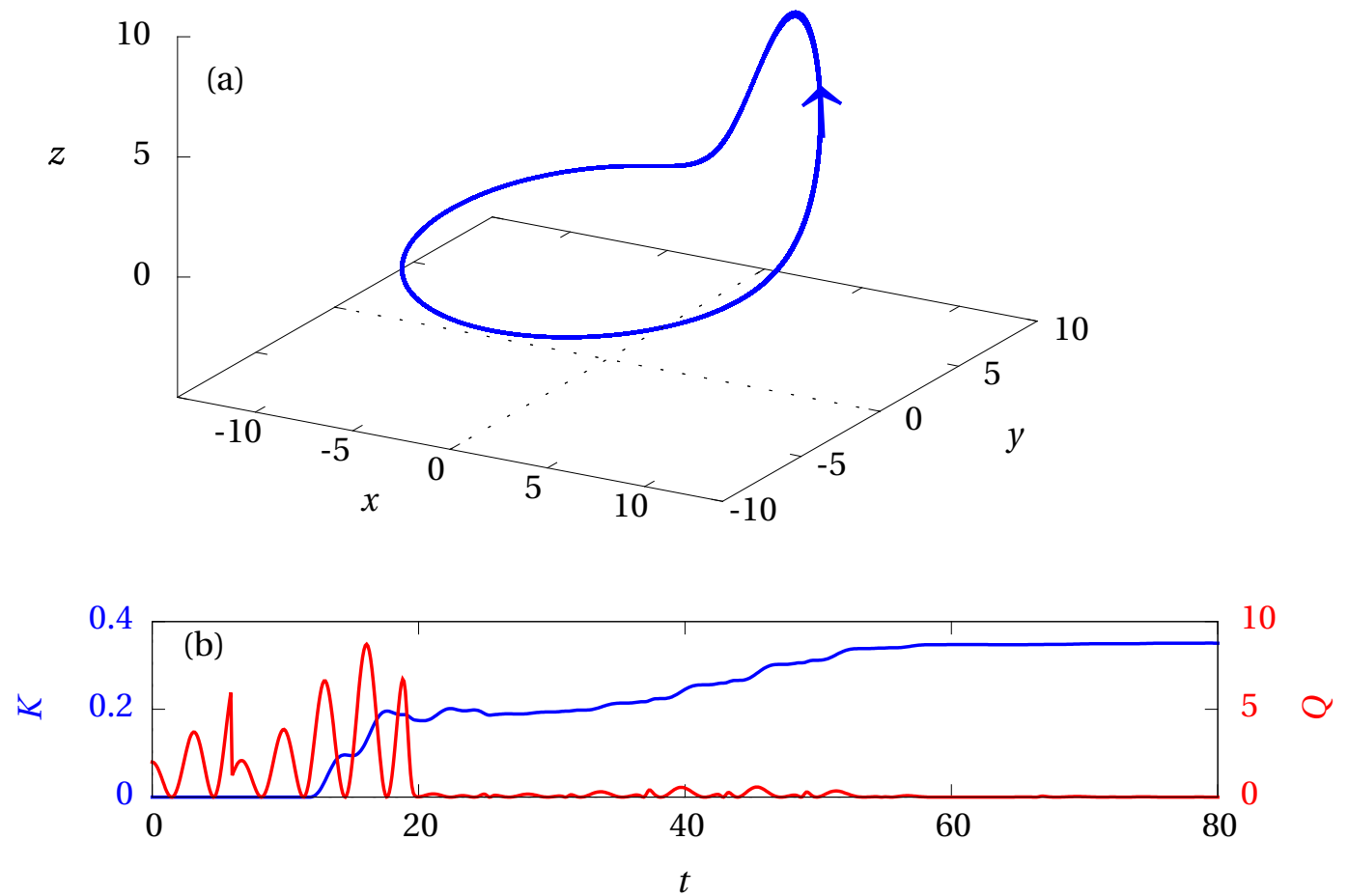

Figure 8.6.: Adaptive control of an unstable periodic orbit in the Rössler attractor Eq. (8.16). (a) Phase portrait (after a transient time of 150 time units). (b) Time series of $K(t)$ with adaptive control given by Eq. (8.18) as blue curve. The curve shows the goal function $Q$. Parameters: $a=0.2, b=0.2, \mu=6.5, \gamma=0.1, \tau=5.91679$. Figure from [Lehnert et al., 2011b]. 


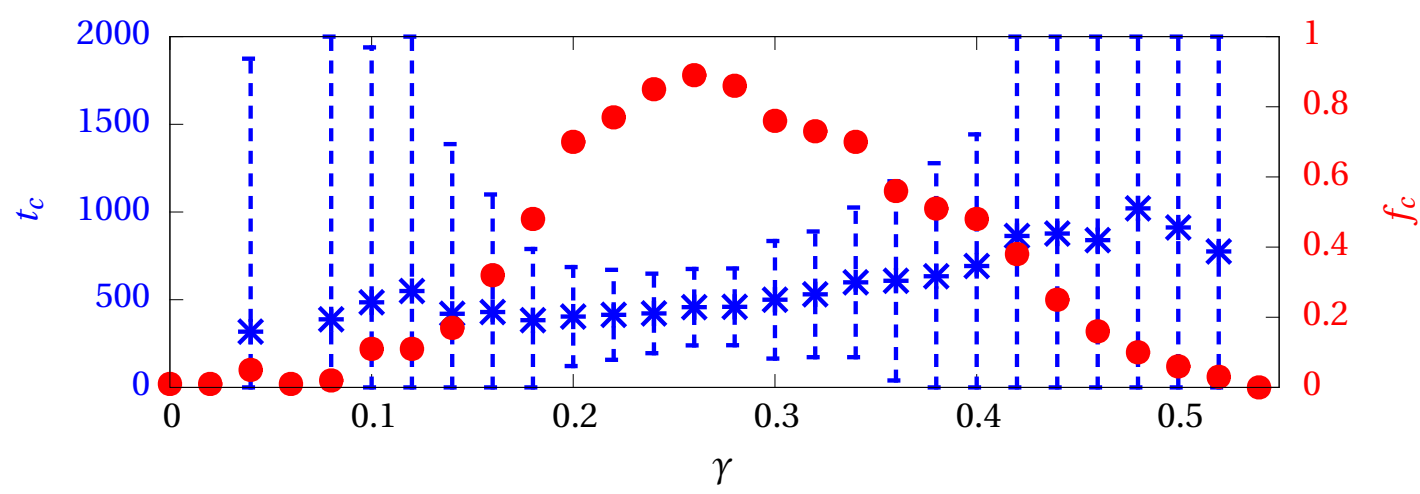

Figure 8.7.: Adaptive control of the Rössler system. Red circles: fraction of realizations $f_{c}$ where the adaptive control algorithm stabilized the orbit versus the adaptation gain $\gamma$; blue crosses: Average time $t_{c}$ after which the control goal is reached versus $\gamma$; dotted blue lines: error bars (standard deviation) corresponding to $t_{c}$. Other parameters as in Fig. 8.6. Total number of realizations for each value of $\gamma$ : 100. Figure from [Lehnert et al., 2011b].

$\langle y(0)\rangle=\langle z(0)\rangle=0$, respectively, and the standard deviations are $\sigma_{x(0)}=\sigma_{y(0)}=\sigma_{z(0)}=1$. It is assumed that the control goal is reached at time $t_{c}$ if the following condition holds: $\langle Q\rangle \equiv{ }_{t_{c}}^{t_{c}-2 \tau} Q\left(t^{\prime}\right) d t^{\prime}<0.002 \tau$.

Figure 8.7 depicts $f_{c}(\gamma)$ (red circles) and $t_{c}(\gamma)$ (blue crosses) demonstrating that the optimal adaptation gain is around $\gamma=0.26$. For $\gamma$ close to this value, the algorithm converges fast and reliably. Accordingly, the standard deviation of $t_{c}$ is small.

This demonstrates that for appropriate values of $\gamma$, the chaotic dynamics can be controlled.

\subsection{CONCLUSION}

In summary, the SG-method can be used to extent TDFC and ETDFC to an adaptive controller. We have demonstrated this adaptive TDFC by tuning the feedback gain in the stabilization of an unstable focus in a generic model and in the control of an unstable periodic orbit embedded in a chaotic attractor. The method is robust to different initial conditions and noise. Adaptive method may especially be useful for systems with unknown or slowly changing parameters where the domains of stability in parameter 
space are unknown. As shown by a simulation with a drifting bifurcation parameter $\lambda$, the adaptive TDFC is able to follow such slow parameter drifts.

Given the great interest in TDFC in the last two decades, several other adaptive methods to tune the delay time [Kittel et al., 1995; Chen and Yu, 1999; Herrmann, 2001; Pyragas and Pyragas, 2011] and/or the coupling strengths [Nakajima et al., 1997; Pyragas and Pyragas, 2013; Lin et al., 2010; Selivanov et al., 2015] have been developed. In [Selivanov et al., 2012] we developed an alternative method to adapt the coupling strength in an unstable focus with TDFC. Compared to method presented in Sec. 8.2 this method has the advantage that it is globally stable which can be shown analytically with the help of a Lyapunov-Krasovskii functional. However, the calculation of the adaptation law requires advanced techniques as the discrete Lyapunov functional approach [Gu et al., 2003]. Furthermore, the system's governing equation have to be known.

In summary, the advantage of the scheme presented here is its simplicity. Neither does it require the knowledge of the system's governing equation [Pyragas and Pyragas, 2013; Selivanov et al., 2015], nor does it involve averaging or integrating over time series [Chen and Yu, 1999; Pyragas and Pyragas, 2011].

Note, however, that the adaptive controller presented here finds - dependent on initial conditions - any appropriate feedback gain but not necessarily the optimal one. For an adaptive method optimizing for speed of convergence to the periodic orbit or fixed point see [Pyragas and Pyragas, 2013]. Similarly, Bick et al. [2012] suggest a scheme for tuning control parameters in predictive feedback control [Polyak, 2005] to an optimal value.

We have studied the adaptation of the coupling strength in two other contexts: In [Guzenko et al., 2013b,a], we considered the control of network motifs of delay-coupled chaotic Rössler systems. We showed that by simultaneously tuning the coupling strength and the self-feedback strength, local stabilization of unstable periodic orbits embedded in the Rössler attractors and global synchronization of these orbits is possible at the same time. In [Plotnikov et al., 2015], we demonstrated that the adaptive control of zero-lag synchronization in networks of coupled FitzHugh-Nagumo systems with heterogeneous threshold parameters is feasible. 


\section{9 | AdAPTIVE CONTROL OF Cluster STATES IN NETWORK MOTIFS}

In the previous Chapter, I have discussed the adaptive control of uncoupled systems and have shown that the time-delayed feedback control (TDFC) can be turned into an adaptive scheme with the help of the speed-gradient (SG) method. The aim of this Chapter is to develop an adaptive controller for network motifs. More specifically, we want to control cluster synchronization in motifs of delay-coupled Stuart-Landau oscillators. For this system, Choe et al. [2010] showed that the phase $\beta$ of the complex coupling strength $\sigma=K \exp (i \beta)$ is a crucial parameter when stabilizing cluster states. For a recapitulation of their results see Sec. 5.2. In [Selivanov et al., 2012; Schöll et al., 2012b,a], we developed an adaptive control of $\beta$ with the goal to realize a state with $M$-clusters separated by a phase lag of $2 \pi / M$. This Chapter discusses the results of the method and also its limitations in the case of larger networks. As before, we apply the SG method (see Sec. 7.1) to construct the adaptive controller.

\subsection{PHASE OF THE COMPLEX COUPLING STRENGTH}

The complex coupling constant $\sigma$ that arises from the complex state variables in networks of Stuart-Landau oscillators consists of an amplitude $K$ and a phase $\beta$. Similar coupling phases arise naturally in systems with all-optical coupling [Schikora et al., 2006; Flunkert and Schöll, 2007]. Furthermore, they can be used to overcome the odd-number limitation of time-delay feedback control [Fiedler et al., 2007; Schikora et al., 2011] and to anticipate chaos synchronization [Pyragas and Pyragiene, 2008]. In [Choe et al., 2010, 2011; Dahms, 2011; Choe et al., 2014a], it was shown that the value of the coupling phase is a crucial control parameter in these systems, and by adjusting this phase one can deliberately switch between different synchronous oscillatory states of the network; see Sec. 5.2. In [Choe et al., 2012], a rotation of the feedback term, which is closely related 
to a non-zero coupling phase, has been used to stabilize unstable periodic orbits and steady states in coupled Lorenz systems. Furthermore, a non-zero coupling phase can be used to obtain noninvasive control in delayed feedback schemes with arbitrary delays [Choe et al., 2014b].

In order to find an appropriate value of the coupling phase to control cluster states in the Stuart-Landau oscillators one could solve the transcendental characteristic equation that involves the system parameters. However, in practice the exact values of the system parameters are unknown, and analytical conditions can be derived only for special values of the complex coupling phase. An efficient way to avoid these limitations and to automatically find appropriate values of the coupling phase is the use of adaptive control. In the following, we apply the SG to develop such an adaptive control.

\subsection{GOAL FUNCTION FOR CLUSTER SYNCHRONIZATION}

The most challenging part in applying the SG method is to find an appropriate goal function. We suggested an appropriate goal function $Q_{M}$ to control an $M$-cluster state in [Selivanov et al., 2012; Schöll et al., 2012b]:

$$
Q_{M}=1-f_{M}(\varphi)+\frac{N^{2}}{2} \underset{p \mid M, 1 \leq p<M}{ } f_{p}(\varphi),
$$

where $p \mid M$ denotes that $p$ is a factor of $M$. The function

$$
f_{M}(\varphi)=\frac{1}{N^{2}}{ }_{j=0}^{N-1} e^{\mathrm{i} M \varphi_{j}}{ }_{k=0}^{N-1} e^{-\mathrm{i} M \varphi_{k}},
$$

where $\varphi_{j}$ is the phase of the $j$ th oscillator, $j=0, \ldots, N-1$, and $\varphi \equiv\left(\varphi_{0}, \ldots, \varphi_{N-1}\right)$, is a generalization of the Kuramoto order parameter [Kuramoto, 1984] (see also Subsec. 3.3) and approaches unity for an $M$-cluster state. Since $f_{M}=1$ not only holds in the desired $M$-cluster state but also for all divisors $p$ of $M$, a goal function of the form $Q_{M}=$ $1-f_{M}(\varphi)$ would also vanish if the system were in one of the $p$-states. Therefore, the term $p \mid M, 1 \leq p<M f_{p}(\varphi)$ was added in Eq. (9.1) as a penalty term. Figure 9.1 illustrates the impact of the penalty terms with the simple example of the goal function for a network with two nodes: For zero-lag synchronization no penalty term is needed as $Q_{1}$ exhibits only the desired minimum $\varphi_{0}=\varphi_{1}$ (see panel (a), where the blue line marks the minimum). In contrast, $1-f_{2}(\varphi)$, i.e., the goal function for antisynchronization but without 


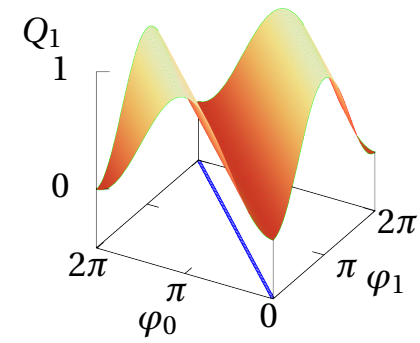

(a)

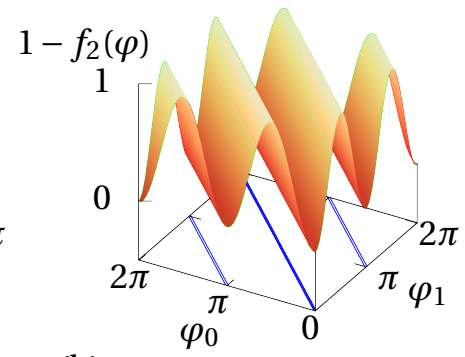

(b)

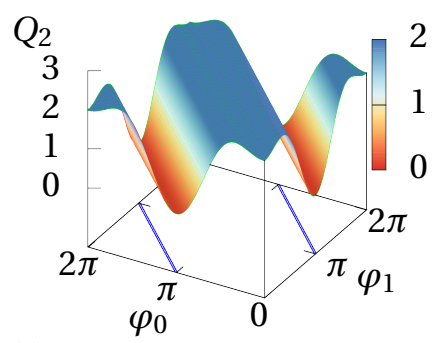

(c)

Figure 9.1.: Goal functions for cluster synchronization. (a) Goal function $Q_{1}$ for zero-lag synchronization. (b) Goal function $1-f_{2}(\varphi)$ for antisynchronization without penalty terms. (c) Goal function $Q_{2}$ for antisynchronization. Blue line: 0-contour line, i.e., minium of the depicted function. $N=2$.

a penalty term, has a minimum for the antisychronized state $\left|\varphi_{0}-\varphi_{1}\right|=\pi$ but also for the zero-lag synchronized state $\varphi_{0}=\varphi_{1}$ (see panel (b)). This side minimum can be prevented by adding the penalty term as shown in panel (c), where $Q_{2}$ is depicted. The factor $1 / N^{2}$ in Eq. (9.2) has been found to be a sensible choice in order to balance the desired minimum of the goal function (9.1) against its side maxima introduced by the penalty terms.

The advantage of goal function (9.1) is that it does not a priori assign each node to a cluster but that the ordering of nodes into clusters is a self organized process. As a result, often nodes cluster together which are already initially close to each other in phase space leading to a fast convergence of the control process. In [Selivanov et al., 2012] other goal functions were suggested which were also successful in the stabilization of an $M$-cluster state. However, due to the just discussed advantage and the fact that goal function (9.1) is the basis of further work on adaptive control which I will present in Chapter 10, I will focus on this function here. 


\subsection{Controlling AN $M$-CLUSter State}

In Eq. (5.45) in Sec. 5.2 a network of $N$ delayed Stuart-Landau oscillators was introduced

$$
\dot{z}_{j}=\left[\lambda_{j}+\mathrm{i} \omega_{j}-\left|z_{j}\right|^{2}\right] z_{j}+K e_{n=0}^{\mathrm{i} \beta{ }_{j n}^{N-1}} G_{j n}(t)\left[z_{n, \tau}-z_{j}(t)\right]
$$

where $z_{j} \in \mathbb{C}$ is the state of the $j$ th oscillator. $\omega_{j}$ and $\lambda_{j}$ are the frequency and the bifurcation parameter of the $j$ th uncoupled node, respectively. In contrast to Sec. 5.2, we consider here also nonidentical nodes. $\sigma=K e^{\mathrm{i} \beta}$ with $K, \beta \in \mathbb{R}$ is the complex overall coupling strength, $\tau$ the delay time, and $\mathbf{G}$ the $N \times N$ coupling matrix (see Sec. 2.3). Here and in the following, delayed variables are denoted by a lower index $\tau$, for example, $z_{j}(t-\tau) \equiv z_{j, \tau}$.

With $r_{j}=\left|z_{j}\right|$ and $\varphi_{j}=\arg \left(z_{j}\right)$, Eq. (9.3) can be rewritten in these amplitude and phase variables

$$
\begin{aligned}
\dot{r}_{j}(t) & =\lambda_{j}-r_{j}^{2} r_{j}+K{ }_{n=0}^{N-1} G_{j n} r_{n, \tau} \cos \beta+\varphi_{n, \tau}-\varphi_{j}-r_{j} \cos \beta, \\
\dot{\varphi}_{j}(t) & =\omega_{j}+K_{n=0}^{N-1} G_{j n} \frac{r_{n, \tau}}{r_{j}} \sin \beta+\varphi_{n, \tau}-\varphi_{j}-\sin \beta .
\end{aligned}
$$

The SG algorithm in the form of Eq. (7.4) reads [Selivanov et al., 2012]

$$
\begin{aligned}
\dot{\beta}= & -\gamma \underset{j}{ } \frac{\partial Q}{\partial \varphi_{j}} \frac{\partial \dot{\varphi}_{j}}{\partial \beta} \\
= & -\gamma K{ }_{j=0}^{N-1 N-1} \quad k=0 \quad p \mid M, 1 \leq p<M \\
& \times{ }_{n=0}^{N-1} G_{j n} \frac{r_{n, \tau}}{r_{j}} \cos \left(\beta+\varphi_{n, \tau}-\varphi_{j}\right)-\cos (\beta)
\end{aligned}
$$

where we used Eq. (9.1) as a goal function and identified $\beta$ with the control variable $\mathbf{u}$. As we consider here the one dimensional case of Eq. (7.4) $\gamma>0$ is a constant.

In the following, I will discuss as an example the control of a 3-cluster state. Recall that an $M$-cluster state might, depending on $M$ and $N$, correspond to several different 

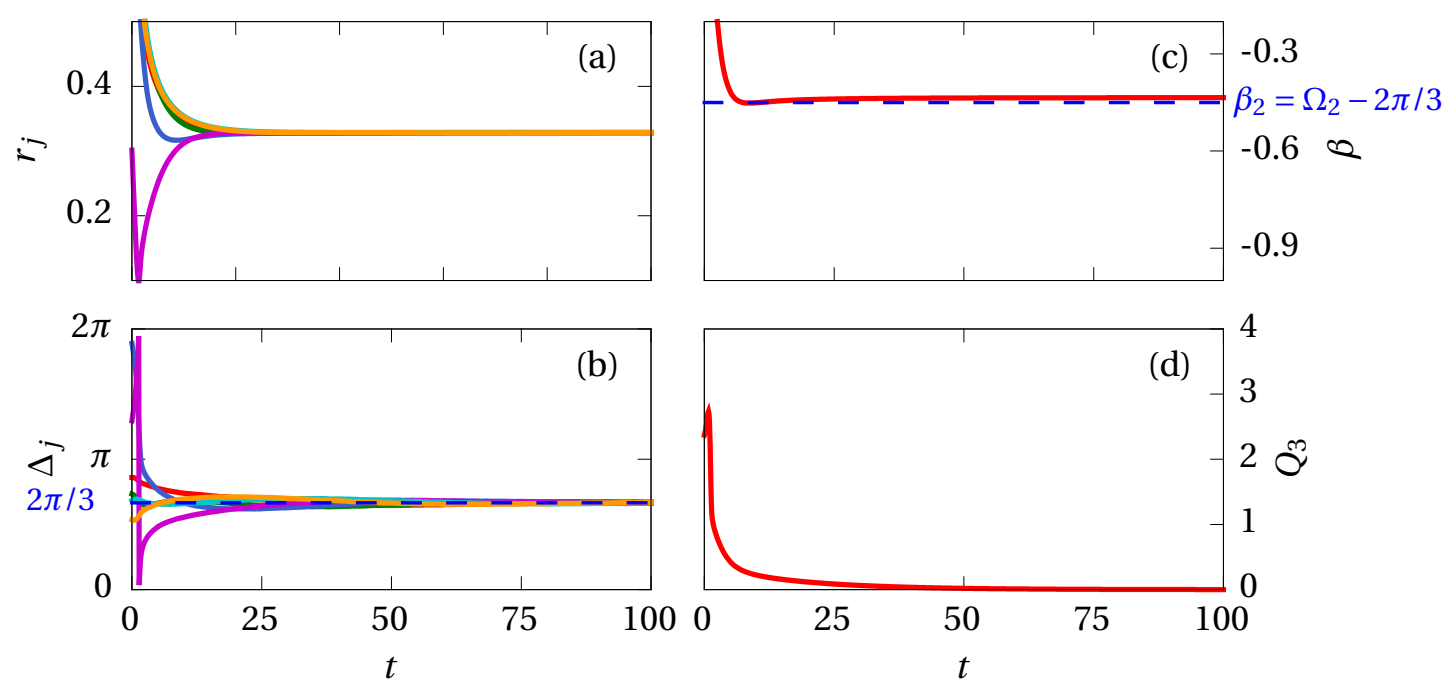

Figure 9.2.: Adaptive control of 3-cluster $(m=2,4)$ state in a network of 6 nodes according to Eq. (9.5). (a) Absolute values $r_{j}=z_{j}$. (b) Phase differences $\Delta_{j}=\varphi_{j+1}-\varphi_{j}$; blue dashed line: phase difference $\Delta_{j}=4 \pi / 3$ corresponding to a 3 -cluster state with $m=2$. (c) Temporal evolution of $\beta$; blue dashed line: $\beta_{2}$ with $\Omega_{2}=1.03$. (d) Goal function. $K=0.08, \tau=0.52 \pi, N=6, \gamma=1, \omega_{i=0, \ldots, N-1}=1, \lambda_{i=0, \ldots, N-1}=0.1$. Figure reproduced from [Selivanov et al., 2012]. 

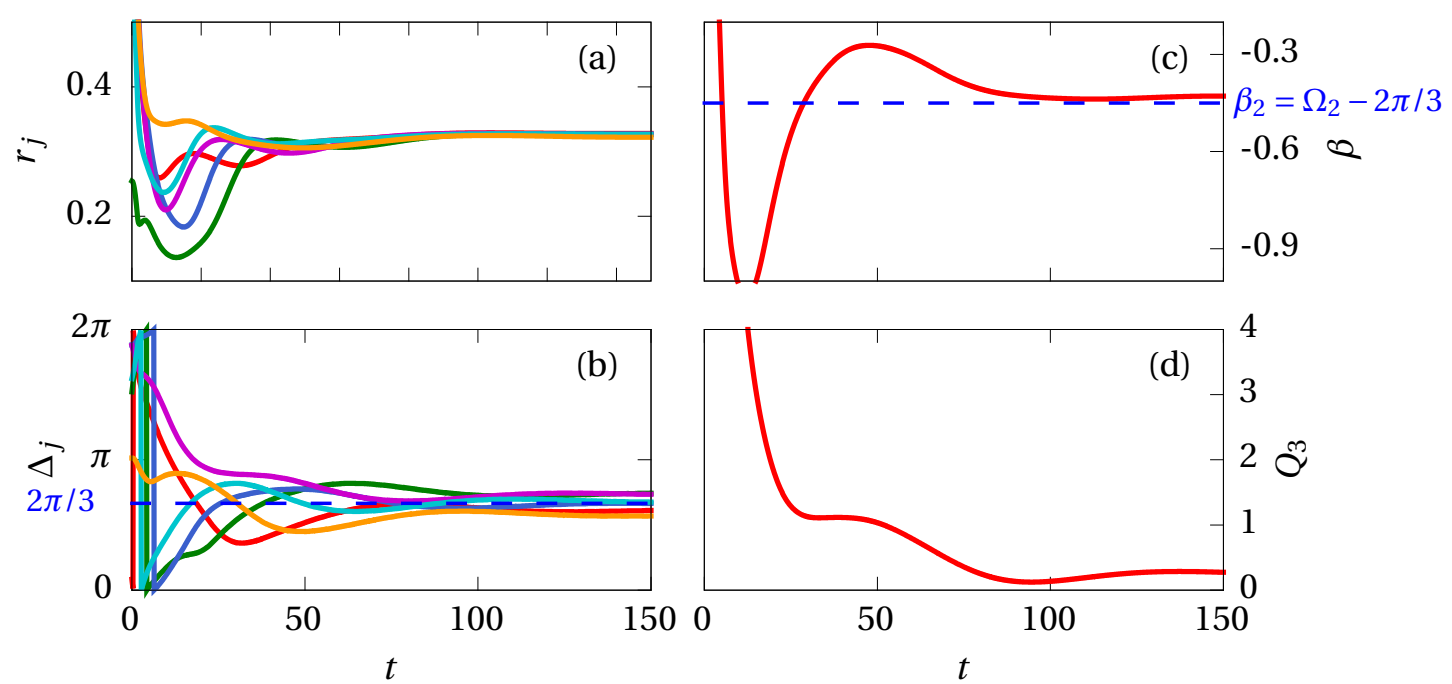

Figure 9.3.: Same as in Fig. 9.2 but for nonidentical oscillators. Parameters $\lambda_{j}$ and $\omega_{j}$ are chosen from a Gaussian distribution with $1 \%$ standard deviation and mean values $\lambda=0.1$ and $\omega=1$, respectively. Here: $\omega_{0}=1.0192, \omega_{1}=1.0093, \omega_{2}=0.9780, \omega_{3}=$ 0.9982, $\omega_{4}=0.9799, \omega_{5}=0.9930, \lambda_{0}=0.0988 \lambda_{1}=0.1017, \lambda_{2}=0.1004, \lambda_{3}=0.09914$, $\lambda_{4}=0.1008, \lambda_{5}=0.1000$. Figure reproduced from [Selivanov et al., 2012]. 

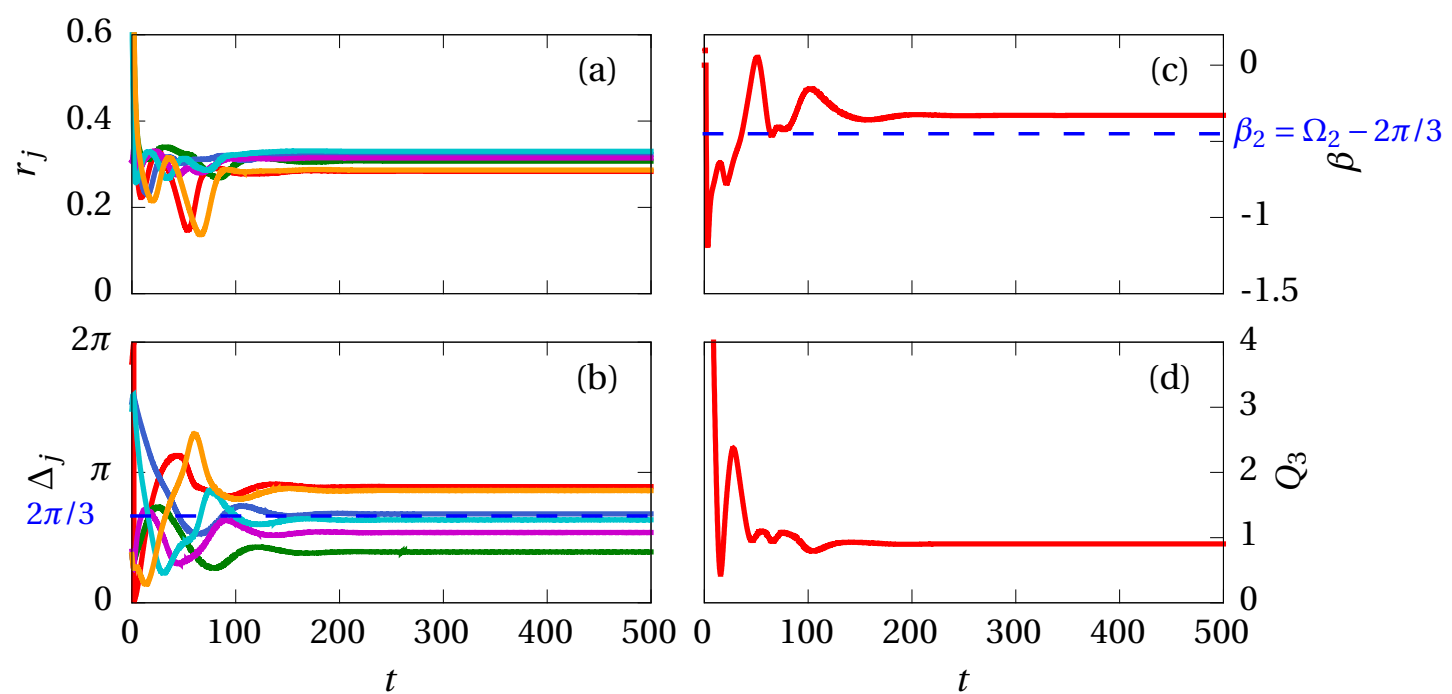

Figure 9.4.: Same as in Fig. 9.3 but for $5 \%$ standard deviation, i.e., $\omega_{0}=0.9395, \omega_{1}=$ 0.9288, $\omega_{2}=1.0495, \omega_{3}=0.9796, \omega_{4}=1.0160, \omega_{5}=0.9909, \lambda_{0}=0.0975, \lambda_{1}=0.0956$, $\lambda_{2}=0.1090, \lambda_{3}=0.1018, \lambda_{4}=0.0936, \lambda_{5}=0.1147$. Figure reproduced from [Selivanov et al., 2012].

states with different $m$ where the phase difference between neighboring nodes is given by $2 \pi m / N$ and $M$ relates to $m$ according to $M=\operatorname{lcm}(m, N) / m$ (see Sec. 5.2); lcm stands for the least common multiple. Thus, the 3 -cluster state in a network of six nodes corresponds of the states $m=2$ and $m=4$. The adaptive controller does not distinguish between the different states with the same $M$ but different $m$. So we anticipate that the system goes to either of these states depending on the initial conditions.

Figure 9.2 presents the results of a numerical simulation of system (9.3) with adaptation law (9.5) for $M=3$ and identical nodes. The topology is a unidirectional ring $\left(G_{i, j}=\right.$ $\delta_{(i+1)} \bmod N, j$ where $\delta$ is the Kronecker delta, see Sec. 2.3.1) with $N=6$ nodes. The model parameters are chosen as in [Choe et al., 2010], i.e., $\omega=1, \lambda=0.1$. In panel (a) it can be seen that the radii $r_{j}$ of all nodes converge after about 40 time units. Panel (b) shows that the phase differences $\Delta_{j}=\varphi_{j}-\varphi_{j+1}$ approach $4 \pi / 3$, which corresponds to a 3-cluster state with $m=2$. Panel (c) depicts the evolution of $\beta$. The blue dashed line represents the value of the coupling phase $\beta_{m=2}=\Omega_{m=2} \tau-2 \pi / 3$, for which stability was shown analytically in [Choe et al., 2010]. It can be seen that the adaptively adjusted phase comes close to this value. In other words, even without knowing the exact values of the system parameters, the SG algorithm yields an adequate value of $\beta$ that stabilizes the target state 
of synchronization in a 3-cluster state. Panel (d) shows that the goal function (9.1) indeed approaches zero.

So far we considered identical nodes. However, in experimental situations the nodes are always at least slightly nonidentical. In the following we want to test the robustness of our method towards such heterogeneities. For Fig. 9.3 we repeat our simulation, but chose the parameters $\lambda_{j}$ and $\omega_{j}$ from a Gaussian distribution with $1 \%$ standard deviation. Obviously, the control is still successful; though the transient time until the control goal is reached increases. Due to the heterogeneities of the nodes small deviations of the phase differences $\Delta_{j}=\varphi_{j}-\varphi_{j+1}$ from the value $4 \pi / 3$ and of the goal function from the value 0 , respectively, exist. Perfect synchronization can only be expected for identical nodes because only in this case the cluster solution is an invariant manifold of system (9.3). If the heterogeneities are further increased, i.e., $\lambda_{j}$ and $\omega_{j}$ are drawn from a Gaussian distribution with $5 \%$ standard, the control is still successful, however, the transient time further grows as shown in Fig. 9.4. As expected, the deviations of $\Delta_{j}=\varphi_{j}-\varphi_{j+1}$ and $Q$ from the values $4 \pi / 3$ and 0 , respectively, increase as well but are still small enough to consider the control as successful.

\subsection{LINEAR STABILITY ANALYSIS}

In this Section, a linear stability analysis is performed of system (9.4) with adaptation law (9.5). The aim of this analysis is to understand the mechanism behind the control of cluster states. The main question is whether the adaptation law tunes the value of $\beta$ to a value for which the system with or without adaption shows stable cluster synchronization. The other possibility is that introducing the adaptation law changes the system in such a way that cluster synchronization is now stable for values of $\beta$ for which it is unstable in the original system.

We linearize Eq. (9.4) with adaptation law (9.5) at the goal state

$$
\begin{aligned}
r_{j} & =r_{0, m}, \\
\varphi_{j} & =\Omega_{m} t+\frac{2 \pi m}{N} j, \\
\beta & =\beta^{*},
\end{aligned}
$$

where $\beta^{*}$ is some appropriate value of $\beta$ obtained by adaptation law (9.5). 
In the following, the linear stability analysis conducted by Choe et al. [2010] is extended by including the additional degree of freedom the system has due to the adaptation of $\beta$. A variational equations can be derived using the ansatz $r_{j}(t)=r_{0}\left[1+\delta r_{j}(t)\right], \varphi_{j}(t)=$ $\Omega t+j \frac{2 \pi m}{N}+\delta \varphi_{j}(t)$ and $\beta(t)=\beta^{*}+\delta \beta(t)$. Substituting this ansatz in Eqs. (9.4) and (9.5) yields for the linear order of $\delta r_{j}$ and $\delta \varphi_{j}$

$$
\begin{aligned}
& \begin{array}{l}
\delta \dot{r}_{j} \\
\dot{\delta} \dot{\varphi}_{j}
\end{array} \quad=\quad \begin{array}{ccc}
-2 r_{0}^{2} & 0 & \delta r_{j} \\
0 & 0 & \delta \varphi_{j}
\end{array} \\
& { }_{-K} \quad{ }_{n} G_{j n} \cos \Phi_{n}^{j}-{ }_{n} G_{j n} \sin \Phi_{n}^{j} \quad \delta r_{j} \\
& { }_{n} G_{j n} \sin \Phi_{n}^{j} \quad{ }_{n} G_{j n} \cos \Phi_{n}^{j} \quad \delta \varphi_{j}
\end{aligned}
$$

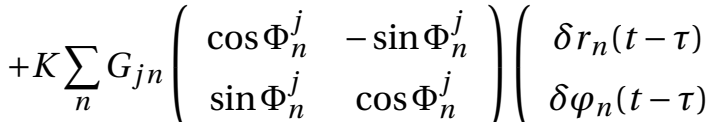

$$
\begin{aligned}
& +K \quad \begin{array}{c}
{ }_{n} G_{j n}\left(-\sin \Phi_{n}^{j}+\sin \beta^{*}\right) \\
\quad{ }_{n} G_{j n}\left(\cos \Phi_{n}^{j}-\cos \beta^{*}\right)
\end{array} \quad \delta \beta,
\end{aligned}
$$

where $\Phi_{n}^{j}=\beta^{*}-\Omega \tau+(n-j) \frac{2 \pi m}{N}$, while for $\dot{\delta} \beta$ we find

$$
\begin{aligned}
\dot{\delta \beta}= & -\gamma K_{j=0 \quad k=0}^{N-1 N-1} \quad p \mid M, 1 \leq p<M \\
& \times{ }_{n=0}^{N-1} G_{j n} \cos \left(\Phi_{n}^{j}\right)-\cos \left(\beta^{*}\right) \quad\left(\delta \varphi_{k}-\delta \varphi_{j}\right) \\
& \left.-\gamma K_{j=0}^{N-1 N-1} \quad k \quad p(k-j) \frac{2 \pi m}{N}\right]-\frac{2 M^{2}}{N^{2}} \\
& \times{ }_{n=0}^{N-1} G_{j n} \quad \cos \left(\Phi_{n}^{j}\right)-\cos \left(\beta^{*}\right) \quad\left(\delta r_{n, \tau}-\delta r_{j}\right) \\
& +\sin \left(\Phi_{n}^{j}\right)\left(\delta \varphi_{n, \tau}-\delta \varphi_{j}\right)+\sin \left(\Phi_{n}^{j}\right)-\sin \left(\beta^{*}\right) \delta \beta .
\end{aligned}
$$

Making use of ${ }_{k} \sin [p(k-j) 2 \pi m / N]=0$ if $2 p \pi m / N \notin \mathbb{N}$, we obtain 


$$
\begin{aligned}
\dot{\delta \beta}= & -\gamma K_{j=0 \quad k=0}^{N-1 N-1} \quad p \mid M, 1 \leq p<M \\
& \times{ }_{n=0}^{N-1} G_{j n} \cos \left[p(k-j) \frac{2 \pi m}{N}\right]-\frac{2 M^{2}}{N^{2}}
\end{aligned}
$$

In the following, we want to apply the master stability approach to Eq. (9.7) to separate the transversal from the longitudinal directions. As in the case of the standard master stability function (MSF), we write the variational equations in a more compact equation and, then, block diagonalize this equation; see Sec. 3.2 for the standard MSF and Sec. 5.2 for the MSF for cluster states of the Stuart-Landau oscillator. We will first study a general topology before considering a unidirectional ring. It will turn out that for unidirectional ring topologies $\delta \beta$ can be treated as a constant. Therefore, we do not include the variational equation for $\delta \beta$ in our compact equation.

Writing Eq. (9.7) in its compact version yields

$$
\dot{\boldsymbol{\xi}}_{j}=\mathbf{J}_{0} \boldsymbol{\xi}_{j}-K \Psi_{j} \boldsymbol{\xi}_{j}+K{ }_{n} G_{j n} \mathbf{R}_{n j} \boldsymbol{\xi}_{n}(t-\tau)+K \mathbf{b}_{j} \delta \beta,
$$

where $\boldsymbol{\xi}_{j}=\left(\delta r_{j}, \delta \varphi_{j}\right) . \mathbf{J}_{0}$ is the Jacobian describing the local dynamics

$$
\mathbf{J}_{0}=\begin{array}{cc}
-2 r_{0}^{2} & 0 \\
0 & 0
\end{array} .
$$

The matrices $\boldsymbol{\Psi}$ and $\mathbf{R}$ and the vector $\mathbf{b}$ are given by

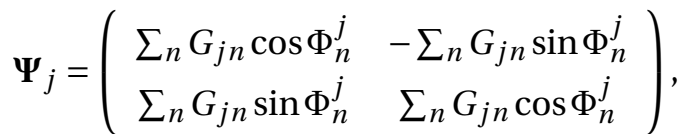

$$
\begin{aligned}
& \mathbf{R}_{n j}=\begin{array}{cc}
\cos \Phi_{n}^{j} & -\sin \Phi_{n}^{j} \\
\sin \Phi_{n}^{j} & \cos \Phi_{n}^{j}
\end{array},
\end{aligned}
$$


and

$$
\mathbf{b}_{j}=\begin{gathered}
{ }_{n} G_{j n}\left[-\sin \Phi_{n}^{j}+\sin \beta^{*}\right] \\
{ }_{n} G_{j n}\left[\cos \Phi_{n}^{j}-\cos \beta^{*}\right]
\end{gathered},
$$

respectively.

Let us now assume that the topology is given by a unidirectional $\operatorname{ring}\left(G_{i j}=\delta_{(i+1) \bmod N, j}\right.$, see Sec. 2.3.1). Then, Eq. (9.9) reads

$$
\begin{aligned}
\dot{\delta \beta}= & -\gamma K_{j=0 \quad k=0 \quad p \mid M, 1 \leq p<M}^{N-1 N-1} p^{2} \cos p(k-j) \frac{2 \pi m}{N}-\frac{2 M^{2}}{N^{2}} \\
& \times \cos \left(\Phi_{m}\right)-\cos \left(\beta^{*}\right)\left(\delta \varphi_{k}-\delta \varphi_{j}\right) \\
= & 0,
\end{aligned}
$$

with $\Phi_{m}=\beta^{*}-\Omega \tau+2 \pi m / N$. For the last step we used ${ }_{k} \cos [p(k-j) 2 \pi m / N]=0$ if $2 p \pi m / N \notin \mathbb{N}$ and $\quad k, j\left(\delta \varphi_{k}-\delta \varphi_{j}\right)=0$. From $\dot{\delta} \beta=0$ follows that $\delta \beta$ can be treated as constant.

Equation (9.10) reads for a unidirectional ring

$$
\dot{\boldsymbol{\xi}}=\mathbb{1}_{N} \otimes\left(\mathbf{J}_{0}-K \mathbf{R}_{m}\right) \boldsymbol{\xi}+K\left(\mathbf{G} \otimes \mathbf{R}_{m}\right) \boldsymbol{\xi}(t-\tau)+K \mathbf{e} \otimes \mathbf{b}_{m} \delta \beta,
$$

where $\boldsymbol{\xi}=\left(\boldsymbol{\xi}_{0}, \ldots, \boldsymbol{\xi}_{N-1}\right), \mathbb{1}_{N}$ is the $N$-dimensional identity matrix and $\mathbf{e}$ is an $N$-dimensional vector with all entries equal to one, i.e., $\mathbf{e}=(1, \ldots, 1) . \mathbf{R}_{m}$ is obtained from $\mathbf{R}_{n j}$ by substituting $G_{i j}=\delta_{(i+1) \bmod N, j}$

$$
\mathbf{R}_{m}=\begin{array}{cc}
\cos \Phi_{m} & -\sin \Phi_{m} \\
\sin \Phi_{m} & \cos \Phi_{m}
\end{array} .
$$

Analogously, the vector $\mathbf{b}_{m}$ is obtained from $\mathbf{b}_{j}$

$$
\mathbf{b}_{m}=\begin{gathered}
-\sin \Phi_{m}+\sin \beta^{*} \\
\cos \Phi_{m}-\cos \beta^{*}
\end{gathered} .
$$

Equation (9.16) consists of $2 N$ coupled variational equations. In the following, we want 
to decouple these equations by block diagonalizing $\mathbf{G}$ which corresponds to the derivation of a master stability equation (MSE); see [Pecora and Carroll, 1998] and Sec. 3.2 for the standard procedure of driving a MSE. Let $\mathbf{S}$ be the matrix diagonalizing $\mathbf{G}$, i.e., $\mathbf{D}_{\mathbf{G}}=\mathbf{S G S}^{-1}$, where $\mathbf{D}_{\mathbf{G}}$ is a diagonal matrix with the eigenvalues of $\mathbf{G}$ on its diagonal. In the case considered here of a unidirectional ring $\mathbf{S}^{-1}$ is given by

$$
S_{j n}^{-1}=\exp \frac{2 \pi j n \mathrm{i}}{N}, \quad j, n=0, \ldots, N-1,
$$

and $\mathbf{S}$ by

$$
S_{j n}=\frac{1}{N} \exp \frac{-2 \pi j n \mathrm{i}}{N}, \quad j, n=0, \ldots, N-1 ;
$$

see also Sec. 2.3.1 for the eigenvectors of a unidirectional ring. Note that we did not normalize the columns of $\mathbf{S}^{-1}$. However, $\mathbf{S}^{-1} \mathbf{S}=\mathbb{1}_{N}$ holds. This choice of $\mathbf{S}^{-1}$ leads to a consistency of the longitudinal variational equation derived with the help of the MSF with the variational equation directly obtained from Eq. (9.6) as will be shown later.

Equation (9.16) can be block diagonalized with respect to the coupling matrix by multiplication with the matrix $\mathbf{S} \otimes \mathbb{1}_{2}$ from the left yielding

$$
\dot{\tilde{\boldsymbol{\xi}}}=\mathbb{1}_{N} \otimes\left(\mathbf{J}_{0}-K \mathbf{R}_{m}\right) \tilde{\boldsymbol{\xi}}+K\left(\mathbf{D}_{\mathbf{G}} \otimes \mathbf{R}_{m}\right) \tilde{\boldsymbol{\xi}}(t-\tau)+K \mathbf{S} \otimes \mathbb{1}_{2} \mathbf{e} \otimes \mathbf{b}_{m} \delta \beta,
$$

with $\tilde{\boldsymbol{\xi}}=\mathbf{S} \otimes \mathbb{1}_{2} \boldsymbol{\xi}$, where $\mathbb{1}_{2}$ is the 2-dimensional identity matrix. The first terms of Eq. (9.21) have been rearranged according to the standard procedure of the MSF, for details see Sec. 3.1. Applying rule (B.1) (see appendix B) it follows

$$
\left(\mathbf{S} \otimes \mathbb{1}_{2}\right)\left(\mathbf{e} \otimes \mathbf{b}_{m}\right)=(\mathbf{S e}) \otimes\left(\mathbb{1}_{2} \mathbf{b}_{m}\right)=\boldsymbol{e}_{1} \otimes \mathbf{b}_{m},
$$

where $\boldsymbol{e}_{1}=(1,0, \ldots, 0)$. For the last step, we used $(\mathbf{S e})_{j}={ }_{n} S_{j n}={ }_{n} 1 / N \exp (-2 \pi j n \mathrm{i} / N)=$ $\delta_{j 0}$. Equation (9.21) consists of $N$ decoupled variational equations of the form

$$
\dot{\boldsymbol{\xi}}_{k}(t)=\mathbf{J}_{0}-K \mathbf{R}_{m} \boldsymbol{\xi}_{k}(t)+K v_{k} \mathbf{R}_{m} \boldsymbol{\xi}_{k}(t-\tau)+\delta_{k 0} K \mathbf{b}_{m} \delta \beta, \quad k=0, \ldots, N-1,
$$

where $v_{k}=e^{2 \mathrm{i} k \pi / N}, k=0, \ldots, N-1$, are the eigenvalues of the coupling matrix $\mathbf{G}$ of the unidirectional ring as calculated in Subsec. 2.3.1.

For $k>0$, Eq. (9.23) is identical to the variational equation of the system without adaptation as discussed by Choe et al. [2010]. For $k=0$ an additional term of the form $K \mathbf{b}_{m} \delta \beta$ arises which acts as an inhomogeneity (recall that $\dot{\delta} \beta=0$, thus, we treat $\delta \beta$ as a constant). 
We substitute the ansatz

$$
\begin{aligned}
\delta r_{0} & =c, \\
\delta \varphi_{0} & =\delta \Omega t,
\end{aligned}
$$

into Eq. (9.23) with $k=0$ and $v_{0}=1$, where $c, \delta \Omega \in \mathbb{R}$ are time independent constants. This yields a particular solution of Eq. (9.23) of the form

$$
\begin{aligned}
& \delta r_{0}=\frac{K}{2 r_{0, m}^{2}}\left[\left(-\sin \Phi_{m}+\sin \beta^{*}\right) \delta \beta+\tau \sin \Phi_{m} \delta \Omega\right], \\
& \delta \varphi_{0}=\delta \Omega t
\end{aligned}
$$

where the constant $\delta \Omega$ is given by

$$
\delta \Omega=\frac{K\left(\cos \Phi_{m}-\cos \beta^{*}\right)}{1+K \tau \cos \Phi_{m}} \delta \beta .
$$

Recall that $k=0$ corresponds to the longitudinal eigenvalue and, thus, to perturbations in the synchronization manifold. Here, we find that a perturbation in $\beta$ causes a shift of $\delta r_{0}$ and $\delta \Omega$ in the common radius $r_{0, m}$ and the common frequency $\Omega_{m}$. This is not surprising as the common radius and the common frequency depend on $\beta$ according to Eq. (5.48). Recall that Eq. (5.48) reads for the unidirectional ring

$$
\begin{aligned}
& r_{0, m}^{2}=\lambda-K \cos \beta+K \cos \Phi_{m}, \\
& \Omega_{m}=\omega-K \sin \beta+K \sin \Phi_{m} .
\end{aligned}
$$

If we insert the ansatz

$$
\begin{aligned}
r_{0, m}(\beta) & =r_{0, m}\left(\beta^{*}\right)\left(1+\delta r_{0}\right), \\
\Omega(\beta) & =\Omega\left(\beta^{*}\right)+\delta \Omega, \\
\beta & =\beta^{*}+\delta \beta,
\end{aligned}
$$

into Eq. (9.27) and carry out a Taylor expansion to the first order in $\delta r_{0}, \delta \Omega$, and $\delta \beta$, we recover Eqs. (9.25) and (9.26). Thus, the result obtained in Eq. (9.23) for $k=0$ is consistent with previous results.

For $k>0$, Eq. (9.23) has a solution of the form $\boldsymbol{\xi}_{k}(t)=\boldsymbol{\xi}_{k}(t=0) \exp (\Lambda t)$ where $\Lambda$ is the transversal Floquet exponent which can be obtained from the transcendental equation 


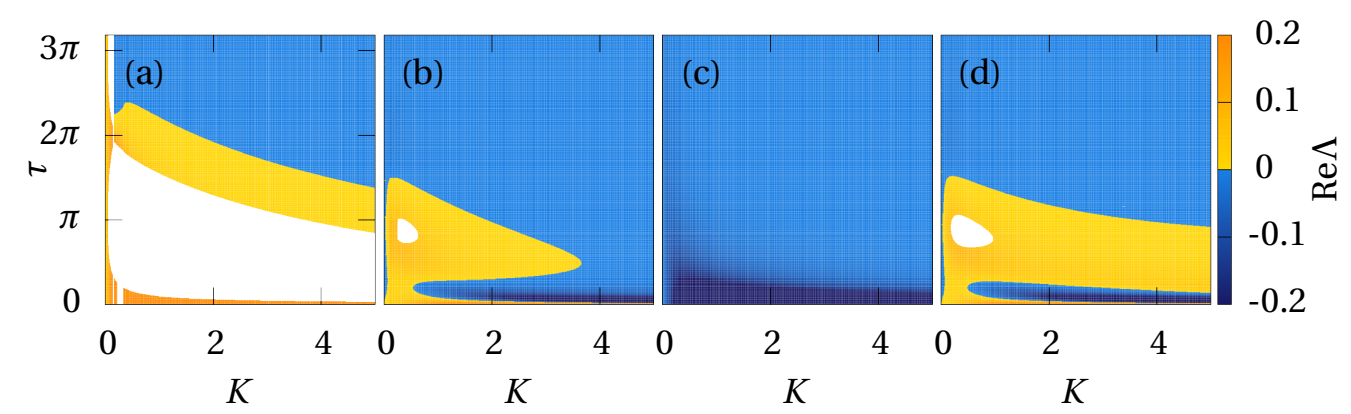

Figure 9.5.: Stability as a function of coupling strength $K$ and delay $\tau$ for (a) $m=0(M=1$, zero-lag synchronization), (b) $m=1$ ( $M=4$, splay state), (c) $m=2(M=2$, antisynchronization), and (d) $m=3$ ( $M=4$, splay-states). In the white areas no solution of Eq. (9.27) with $r_{0, m}^{2}>0$ exists. In all panels, $\beta^{*}=\beta_{m=2}$ according to Eq. (9.33), i.e., the $\beta$ value which stabilizes the $m=2$ state in the whole $(K, \tau)$-plane. $\omega_{i}=1, \lambda_{i}=0.1$, $i=0, \ldots, N-1$. Other parameters as in Fig. 9.3.

$$
0=\operatorname{det}\left[\mathbf{M}-\Lambda \mathbf{I}_{3}\right]
$$

$\mathbf{M}$ is defined as

$$
\mathbf{M}=\begin{array}{ccc}
-2 r_{0}^{2}+K Q_{k} \cos \Phi_{m} & -K Q_{k} \sin \Phi_{m} & 0 \\
K Q_{k} \sin \Phi_{m} & K Q_{k} \cos \Phi_{m} & 0 \\
0 & 0 & 0
\end{array}
$$

with $Q_{k}(\Lambda)=-1+e^{-\Lambda \tau+2 \pi \mathrm{i} k / N}$. We obtained Eq. (9.29) by substituting the ansatz $\xi_{k}(t)=$ $\boldsymbol{\xi}_{k}(t=0) \exp (\Lambda t)$ into Eqs. (9.15) and (9.23); see also the standard procedure of solving linear delay differential equation in Appendix A. If follows that $\Lambda$ is given by

$$
0=\begin{array}{cc}
-2 r_{0}^{2}+K Q_{k}(\Lambda) \cos \Phi_{m}-\Lambda & -K Q_{k}(\Lambda) \sin \Phi_{m} \\
K Q_{k}(\Lambda) \sin \Phi_{m} & K Q_{k}(\Lambda) \cos \Phi_{m}-\Lambda
\end{array}
$$

or by

$\Lambda=0$. 
Condition (9.31) is identical to the condition Choe et al. [2010] found for networks of Stuart-Landau oscillators without adaptive control. $\Lambda=0$ corresponds to a shift in the $\beta$. Such a shift exists because Eq. (9.4) with adaption law (9.5) has a solution for all values of $\beta$, i.e., a line of fixed points exists instead of a single fixed point. This is equivalent to the situation we met in the case of the adaptive control of the coupling strength of the focus in Chapter 8 (see Eq. (8.9) and its discussion). From $\Lambda=0$, we conclude that a change in $\beta$ does not directly affect the stability of Eq. (9.4) with adaption law (9.5) but indirectly because Eq. (9.31) depends on $\beta$ via $\Phi_{m}$. Thus, the stabilizing mechanism of our control method is that $\beta$ is adapted to values where the system with or without control is stable.

In the following, we want to use the stability criterion given by Eq. (9.31) to discuss under which conditions we expect the adaptive control to work well. Prerequisite for the success of the SG method is that a $\beta^{*}$ exists ensuring $\dot{Q}=w\left(z_{0}, \ldots, z_{N-1}, z_{0, \tau}, \ldots, z_{N-1, \tau}, \beta^{*}\right)<$ 0 . This is the case if $\beta^{*}$ stabilizes only the desired state. Otherwise, initial conditions close to one of the other stable states will cause the system to approach this state; the condition $\dot{Q}=w\left(z_{0}, \ldots, z_{N-1}, z_{0, \tau}, \ldots, z_{N-1, \tau}, \beta^{*}\right)<0$ is violated and the control fails. Thus, the question arises whether a $\beta$ exists which ensures monostability. Choe et al. [2010] found that Eq. (9.31) has only negative roots for all values of $K$ and $\tau$ if

$$
\beta_{m} \equiv \Omega_{m} \tau-2 m \pi / N+2 l \pi, l=0, \pm 1, \pm 2, \ldots
$$

is chosen meaning that in this case solution (9.6) is stable for all values $K$ and $\tau$ (see also Eq. (5.52)).

In the following, we want to discuss whether the choice of $\beta_{m}$ not only ensures the existence and stability of the $m$ state but the monostability of this state in which case we expect the adaptive control to work better than in cases where the system is multistable. We explore this question in a unidirectional ring of four nodes and with the control goal to reach antisynchronization, i.e., $M=2$. For a ring of 4 nodes this corresponds to $m=2$. The stability of the different cluster states is determinded by calculating the Floquet exponent with the largest real part according to Eq. (9.31) for $\beta^{*}=\beta_{2}=\Omega_{2} \tau-\pi$. Figure 9.5 shows the result for (a) zero-lag synchronization $(M=1, m=0)$, (b) antisynchronization $(M=2, m=2)$, and (d) splay-states $(M=4, m=1$ and $m=3){ }^{1}$

As anticipated, the antisynchronized state is stable in the whole $(K, \tau)$-plane (see panel

\footnotetext{
${ }^{1}$ Recall that an $M$-cluster state can correspond to several different $m$-states. Furthermore, depending on the parameters, we can find for each $m$-state several different solutions $r_{0, m}$ and $\Omega_{m}$ of Eq. (9.27). To obtain Fig. 9.5, we chose for each value of $K$ and $\tau$ the most stable of these solutions, i.e., the solution whose largest $\operatorname{Re} \Lambda$ had the smallest, if possible negative, value compared to all other solutions.
} 
(c)). However, for large $K$ and $\tau$, the other stable states coexist. Thus, only in a part of the $(K, \tau)$-plane we find monostability of the antisynchronized states. For values of $K$ and $\tau$ from the multistable region and initial conditions close to one of the state with $M \neq 2, \dot{Q}=w\left(z_{0}, \ldots, z_{N-1}, z_{0, \tau}, \ldots, z_{N-1, \tau}, \beta^{*}\right)<0$ will not be fulfilled and the control fails. Though this is only a local discussion, we already can conclude that for large $K$ and $\tau$ the adaptive control will fail for certain initial conditions since a value of $\beta$ ensuring monostability does not exist and, thus, cannot be found adaptively.

\subsection{PERFORMANCE OF THE ADAPTIVE CONTROL OF THE COUPLING PHASE}

The results of Sec. 9.3 showed that the SG method is able to drive the network dynamics into the desired cluster or splay state by adaptively adjusting the coupling phase, where the goal function is chosen according to the corresponding target state. We have, however, used only exemplary values of the coupling parameters $K$ and $\tau$ so far. Furthermore, the results of the linear stability analysis indicated that problems for large $K$ and $\tau$ might arise.

To systematically study the parameter dependency of our method, we calculate the fraction $f_{c}$ of successful realizations. We ran simulations with 20 different initial conditions chosen randomly from the complex interval $[-1,1] \times[-i, i]$ for each oscillator $z_{j}$. A realization is considered to be successful if it approaches the goal state within $t<1000$. Figure 9.6 shows $f_{c}$ in dependence on the coupling strength $K$ and the coupling delay $\tau$ for the goal of (a) zero-lag synchronization, (b) antisynchronization, and (c) a splay state. We observe that the control is successful in a wide parameter range. In the case of zero-lag synchronization and for the splay state $f_{c}$ is close to one for small $K$ and $\tau$, while in the case of antisynchronization $f_{c}$ is for most values of $K$ and $\tau$ smaller than one, indicating that the control of this state is sensitive to initial conditions. Common to all three states is that for large $K$ and $\tau, f_{c}=0$. The region $f_{c}=0$ is separated from the region $f_{c}>0$ by a sharp border which has qualitatively and quantitatively the same form in all three cases.

This border can be explained by considering the transient times. Figure 9.6 (d), (e), and (f) show the time $t_{c}$ until the control goal is reached for zero-lag synchronization, antisynchronization, and a splay state, respectively. Note that only successful realizations are considered. Obviously, the time $t_{c}$ grows with increasing $\tau$ and $K$ until it reaches the value of 1000 at the border. Thus, the border seems to be determined by the maximum time we allow for the control. 

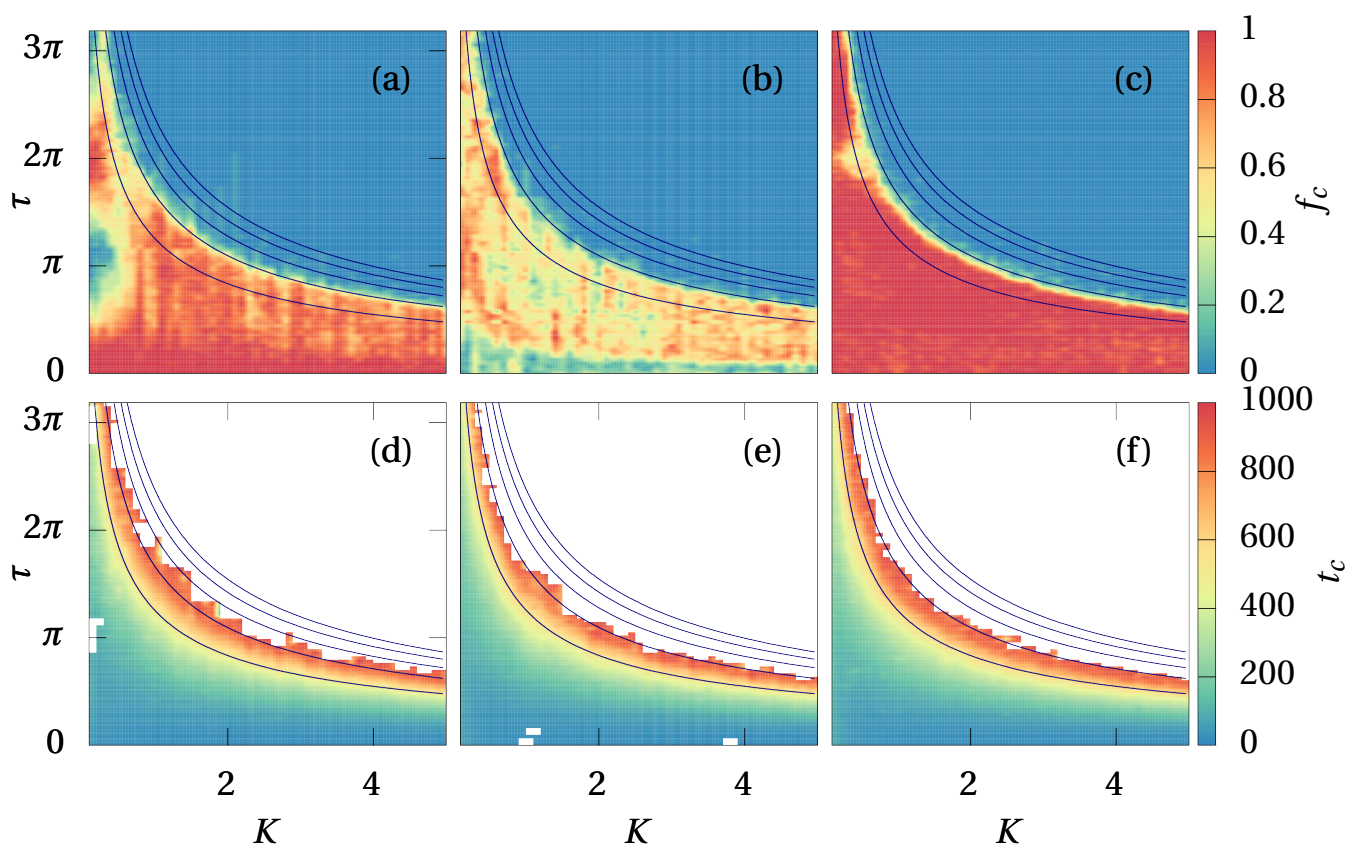

Figure 9.6.: Fraction $f_{c}$ of successfully controlled networks and control time $t_{c}$ as a function of coupling strength $K$ and delay $\tau$ for $(a),(d)$ zero-lag synchronization $(M=1$, $m=0)$; (b), (e) antisynchronization $(M=2, m=2)$; and (c), $(f)$ splay-states $(M=4$, $m=1$ and $m=3)$, respectively. White areas in $(d),(e)$, and $(f)$ correspond to parameter values where no realization was successful within $t<1000$. Dark blue lines correspond to contour lines of $1 /|\operatorname{Re} \Lambda|$ according to Eq. (9.35). $N=4.20$ realizations. Other parameters as in Fig. 9.2. 
$t_{c}$ cannot be obtained analytically because we required for a successful realization that the goal function averaged over 10 unit of time is smaller $3 \times 10^{-5}$. To estimate this, an analytic expression for $Q(t)$ and thus of all phases $\varphi_{j}, j=0, \ldots, N-1$, would be needed. However, $t_{c}$ is in very good approximation proportional to $1 /|\operatorname{Re} \Lambda|$, an expression which can be obtained analytically. Recall that $1 /|\operatorname{Re} \Lambda|$ denotes the time it takes for an initial perturbation to decrease by a factor $1 / e$ and thus is a characteristic time scale of the system.

To obtain an expression $1 /|\operatorname{Re} \Lambda|$ we assume that the control works and $\beta$ approaches $\beta_{m}$ (which is not necessary but often the case). Then, the Floquet exponent with the largest real part is given by

$$
\Lambda=K\left(-1+e^{-\Lambda \tau+2 \mathrm{i} k \pi / N}\right),
$$

where $k$ labels the eigenvalues of the unidirectional ring (see [Choe et al., 2010; Dahms, 2011] and Sec. 5.2.1). Solving for $\Lambda$ yields

$$
\Lambda=-K+\frac{1}{\tau} W\left(K \tau e^{K \tau+2 \mathrm{i} k \pi / N}\right),
$$

with $W$ denoting the Lambert function which is defined as the inverse function of $f(z)=$ $z e^{z}$ for complex variable z [Wright, 1949, 1955; Corless et al., 1996; Asl and Ulsoy, 2003; Amann et al., 2007]. Equation (9.35) constitutes an analytic expression for $\Lambda$. The dark blue lines in Fig. 9.6 mark the contour lines $1 /|\operatorname{Re} \Lambda|=-100,-200,-300,-400,-500$, from bottom to top. Obviously, they have qualitatively the same form as the border between $f_{c}>0$ and $f_{c}=0$ and predict well the increase in $t_{c}$ with growing $K$ and $\tau$. Note that the contour lines become closer to each other as $K$ and $\tau$ increase. Thus, $t_{c}$ grows faster than linearly making the control increasingly time consuming for large $K$ and $\tau$.

\subsection{CONTROLLING SEVERAL PARAMETERS SIMULTANEOUSLY}

The failure of the adaptive control of $\beta$ for large $K$ and $\tau$ raises the question whether it is possible to control these two parameters in addition to $\beta$. In fact, the general form of the SG method as given in Eq. (7.4) allows for controlling several parameters simultaneously. The control vector $u$ in Eq. (7.4) is then given by $u=(\beta, K, \tau)$. We realized such a control in [Schöll et al., 2012b]; the results will be discussed in this Section. In the following the 

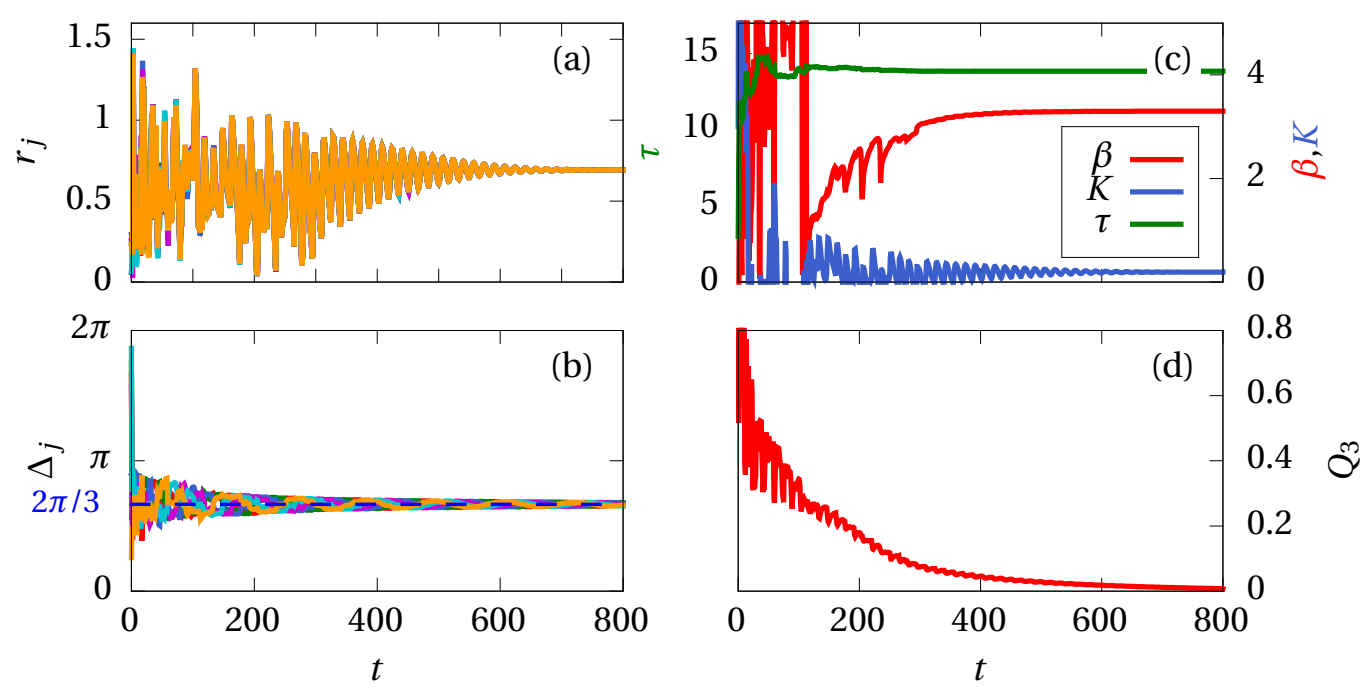

Figure 9.7.: Adaptive control of 3-cluster state in a network of 6 nodes by simultaneously tuning $K, \beta$ and $\tau$ according to Eqs. (9.5), (9.38), and (9.37), respectively. (a) common radii $r_{j}$; (b) phase differences $\Delta_{j}=\varphi_{j}-\varphi_{j+1}$; (c) temporal evolution of control parameters $\beta$ (red line), $K$ (blue line), $\tau$ (green line), (d) goal function. $\gamma_{\beta}=\gamma_{K}=10$, $\gamma_{\tau}=0.05$. Other parameters as in Fig. 9.2. 
feedback gain matrix $\gamma$ is chosen as

$$
\gamma=\begin{array}{ccc}
\gamma_{\beta} & 0 & 0 \\
0 & \gamma_{K} & 0 \\
0 & 0 & \gamma_{\tau}
\end{array}
$$

with $\gamma_{\beta}, \gamma_{K}, \gamma_{\tau}$ being positive real constants.

Using the goal function $Q_{M}$ of Eq. (9.1) yields for $\beta$ the adaptive algorithm given by Eq. (9.5). For $\dot{K}=-\gamma_{K} \frac{\partial}{\partial K} \dot{Q}_{M}$, we obtain

$$
\begin{aligned}
\dot{K}= & -\gamma_{K}{ }_{j=0}^{N-1 N-1} p \ln p \mid d, 1 \leq p<d \\
& \times{ }_{n=0}^{N-1} G_{j n} \frac{r_{n, \tau}}{r_{j}} \sin \left[p\left(\varphi_{k}-\varphi_{j}\right)\right]-\frac{2 d}{N^{2}} \sin \left[d\left(\varphi_{k}-\varphi_{j}\right)\right]
\end{aligned}
$$

and for $\dot{\tau}=-\gamma_{\tau} \frac{\partial}{\partial \tau} \dot{Q}_{M}$

$$
\begin{gathered}
\dot{\tau}=-\gamma_{\tau}^{N-1 N-1} \quad p \sin \left[p\left(\varphi_{k}-\varphi_{j}\right)\right]-\frac{2 d}{N^{2}} \sin \left[d\left(\varphi_{k}-\varphi_{j}\right)\right] \\
\quad \times{ }_{j=0}^{N-1} G_{j n}-\frac{\dot{r}_{n, \tau}}{r_{j}} \sin \left(\beta+\varphi_{n, \tau}-\varphi_{j}\right)-\dot{\varphi}_{n, \tau} \frac{r_{n, \tau}}{r_{j}} \cos \left(\beta+\varphi_{n, \tau}-\varphi_{j}\right) .
\end{gathered}
$$

Figure 9.7 shows the successful control of a 3-cluster state in a network consisting of 6 nodes where appropriate values of $\beta, K$, and $\tau$ are found adaptively. For initial values, $\beta=0, K=3$, and $\tau=3$ were chosen. The adaptation process manages to decrease the values of $K$ and $\tau$ such that the control goal can be reached in a reasonable time of about 600 units of time, while without adaptation of $K$ and $\tau$ the control time would easily exceed 1000 units of time and the control process would be considered as unsuccessful.

\subsection{CONCLUSION}

The adaptive control of $\beta$ allows for controlling cluster states in oscillator networks with time-delayed coupling. The adaptive controller has been developed by means of the 
speed-gradient (SG) method where the goal function has been based on the generalized Kuramoto order parameter. The advantage of this goal function is that it does not require any a priory ordering of nodes. However, the use of other goal functions is also possible but requires that the nodes have to be assigned to the clusters a priori to the control [Selivanov et al., 2012]. In the desired state, e.g., zero-lag synchronization, splay, or cluster states, the goal function vanishes.

By numerical simulations we have shown that the different cluster states can be stabilized, and the coupling phase converges to a value that is predicted to enable stable synchronization in [Choe et al., 2010]. This also works for slightly nonidentical oscillators. A linear stability analysis reveals that the stability of the adaptive system is equal to the stability of the system without adaptation except that an additional Floquet exponent $\Lambda=0$ arises as a result of the shift invariance in the direction of $\beta$. This means that the stabilizing mechanism is such that the control tunes $\beta$ to a value where the system is stable with or without adaptive control. Here, this seems trivial. However, in the next Chapter it will be discussed that other mechanisms might also act in a stabilizing manner in adaptive control problems.

The robustness of the control scheme can be investigated by determining the success rates of the algorithm in dependence on the coupling parameters, i.e., on the coupling strength and on the time delay. We have shown that for small $K$ and $\tau$ the control is successful. The success rate, however, is not equal to $100 \%$ but lower, in particular for the antisynchronized state. The reason is that the system is multistable between different states. This problem is worsened with growing $N$ since the number of possible states is equal to $N$ and, thus, the multistability is increased in large networks.

For large $K$ and $\tau$, the time until the control goal is reached grows rapidly. This time can be estimated analytically by calculating the real part of the largest Floquet exponent whose inverse modulus is proportional to the control time. If it is required that the goal is reached in a given time - which will always be the case in practical applications - the control has to be considered as failing for large $K$ and $\tau$.

The simultaneous control of $K, \tau$, and $\beta$ allows for controlling the system for values of $K$ and $\tau$ which are initially too large for an acceptable control time. The control drives $K$ and $\tau$ to lower values and decreases the time until the control goal is reached. However, the control time remains two orders of magnitude higher than the time scale of the system.

We have to conclude that despite the successful control of, in particular, zero-lag synchronization and the splay state for small $K$ and $\tau$, the control of $\beta$ exhibits two main limitations. First, due the multistability of the systems the control rate can be much 
smaller than $100 \%$ depending on the parameters and the goal state and, second, the control time increases rapidly for large $K$ and $\tau$. Consequently, there is the need for a control method which is more reliable in the sense that it works for different initial conditions for all clusters states, and which is furthermore fast and also applicable to large networks. The adaptive control of the topology discussed in the next Chapter fulfills these criteria. 


\section{0 | AdAptive Topologies}

The previous Chapter introduced a new method to control cluster synchronization by adaptively tuning $\beta$, the phase of the complex coupling strength $\sigma=K \exp (i \beta)$. Although, this method works well for the control of zero-lag synchronization and splay states in small network motifs, it is sensitive to initial conditions when it comes to other cluster states. Furthermore, it is not applicable if the absolute value of the coupling strength $K$ and the delay time $\tau$ become too large since the time to reach the control goal increases rapidly with these parameters. The aim of this Chapter is to find a control method that overcomes these shortcomings. To this end, a control algorithm is designed that changes the topology such that a desired cluster state is reached. Networks which are characterized by an interplay between a changing topology and the dynamics of the nodes are called adaptive networks. To find an algorithm to appropriately adapt the topology, the speed-gradient (SG) method (see Sec. 7.1) is used, which has already proven to be a useful tool for designing adaptive controller of single, uncoupled systems in Chapter 8 and of coupled systems in Chapter 9.

To what extent structure determines function, i.e., which topologies allow for cluster synchronization, is a hot topic of current research [Golubitsky and Stewart, 2002; Kanter et al., 2011b,a; Pecora et al., 2014]. In this Chapter, I will demonstrate that the control of synchronization via the adaptation of the topology provides an easy and self-organized way to generate weighted networks that are able to exhibit cluster synchronization. The topology of these networks will contain some randomness as each control process is started with random initial conditions. However, on average the topology is characterized by common features that enable synchronization and hence yield the desired cluster state. It will be shown that the delay time is a crucial parameter shaping this topology.

An introduction to adaptive networks is given in Sec. 10.1. Section 10.2 extends the goal function utilized in the previous Chapter in order to fit the new problem, i.e., the realization of control of cluster synchronization by adjusting the network topology. The 
adaptive algorithm is introduced and its capability is demonstrated with an example in Sec. 10.3, while its performance is evaluated in Sec. 10.4. In Sec. 10.5, a linear stability analysis is conducted. The topology of the networks after sucessful control is studied in Sec. 10.6. Section 10.7 demonstrates how a network can be constructed which oscillates with a prescribed frequency. Not all links have to be adapted for successful control of cluster synchronization but it suffices to target a subset of links which is discussed in Sec. 10.8. The Chapter concludes with Sec. 10.9.

\subsection{ADAPTIVE NETWORKS}

Previous research in the field of network science focused either on the construction of topologies [Rapoport, 1957; Erdős and Rényi, 1959; Watts and Strogatz, 1998; Albert and Barabasi, 2002; Newman, 2003; Boccaletti et al., 2006b] or on the dynamics on a network with fixed topology [Albert et al., 2000; Dhamala et al., 2004; Chavez et al., 2005; Sorrentino and Ott, 2007; Zigzag et al., 2009; Choe et al., 2010; Kinzel et al., 2009; Lehnert et al., 2011a; Keane et al., 2012]. Recently, adaptive networks attracted a lot of interest as they bring these two aspects together: In such networks the topology evolves according to the state of the system while the dynamics on the network and thus the state is influenced by the topology [Gross and Blasius, 2008].

Figure 10.1 schematically illustrates the idea behind adaptive networks. The state of the nodes affects the topological evolution, which determines the topology. The topology, in turn, has a strong impact on the local dynamics, which governs the evolution of the state of the nodes. Note that the state of the system at time $t$ is given by the state of the nodes and the topology at time $t$, or in the case of a system with delay by the state of the nodes and the topology on the interval $[t-\tau, t]$, where $\tau$ is the maximum delay present in the system (see also Sec. 2.4 for a description of networks with delay). Thus, one major challenge of dealing with adaptive networks is the high dimensionality of the phase space.

Adaptive networks are found as a descriptive tool in all situations where there is an interplay of topology and dynamics. Prominent examples are neural networks where the probability of a neuron to spike highly depends on its input from other neurons. The spiking behavior, in turn, can influence the neural connectivity via synaptic plasticity or via the emergence of new connections. A first model describing this interplay was given in [Hopfield, 1982]. Another example of an adaptive network is swarm dynamics where the decision of the individual agents is influenced by the positions of the other agents, i.e., the topology, while their decisions where to go determines the topology [Huepe et al., 


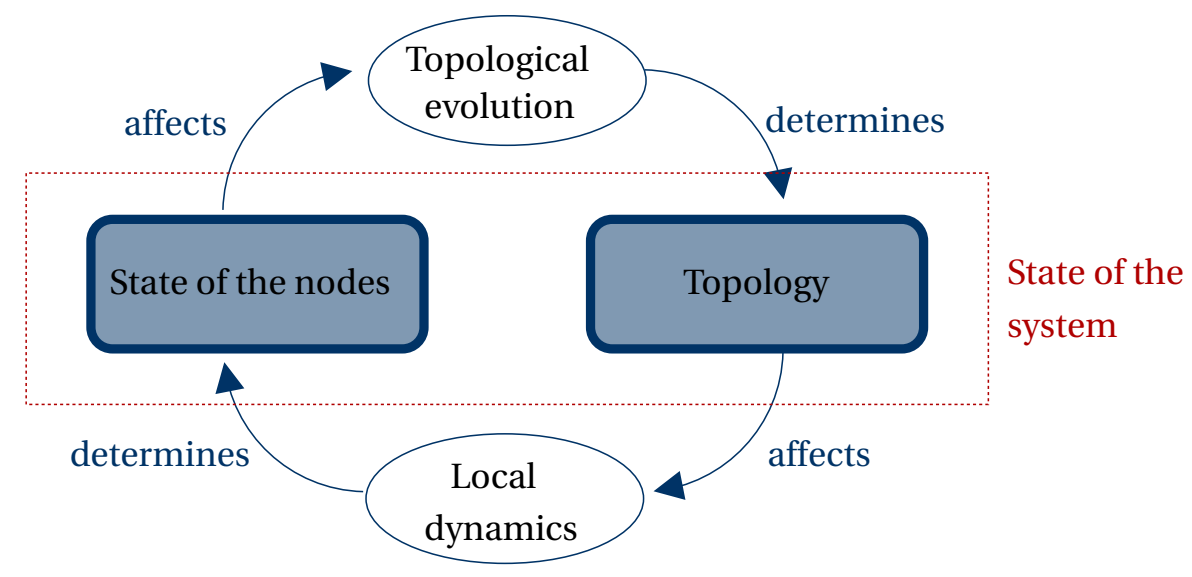

Figure 10.1.: Schematic view of the interplay of topology and local dynamics in a adaptive network. Figure modified from Fig. 1 in [Gross and Blasius, 2008].

2011]. Further examples include communication networks, the network of blood vessels, social network, food webs, and chemical networks; see [Gross and Blasius, 2008] and reference therein.

Despite the success of adaptive networks in the description of all kinds of phenomena observed in nature, little research has focused on how a topology has to be designed to ensure certain dynamics; or in other words how to use the topology to control the dynamics. Control of cluster synchronization by adaptively changing the topology of the network has previously been investigated, to my knowledge, only by a few researchers: Lu et al. consider control of cluster synchronization by means of changing topology. As a limiting restriction for the applicability, their method requires a-priori knowledge to which cluster each node should belong in the final state [Lu and Qin, 2009]. In contrast, for the method introduced in this Chapter, no a-priori ordering of nodes is needed, i.e., it is not necessary to assign each node to a specific cluster in advance; but the final assignment is a built-in consequence of the initially designed goal function.

Furthermore, the majority of algorithms developed to control (mainly in-phase) synchronization by adaptation of the network topology are based on local mechanisms. Most of them are related to Hebb's rule: Cells that fire together, wire together [Hebb, 1949]. The method investigated in this Chapter [Lehnert et al., 2014] uses a global goal function to realize self-organized control and is therefore a powerful alternative and complements existing control schemes. 


\subsection{GOAL FUNCTION}

The main ingredient of the SG method [Fradkov, 2007] is a goal function $Q(z(t), t)$ that vanishes for the target state, and is larger than zero otherwise (see Sec. 7.1). Here, as in Chapter 9, the target state is an $M$ cluster state, i.e., a state consisting of $M$ equally sized clusters with a constant phase lag $2 \pi / M$ between subsequent clusters ${ }^{1}$.

In principle, the goal function presented in the previous Chapter (see Eq. (9.1)) could also be used to control cluster synchronization by means of adapting the topology and, in fact, the results are qualitatively similar to the ones presented here. However, Eq. (9.1) has two drawbacks: First, it only ensures phase synchronization, i.e., the radii of the oscillators do not synchronize, and, second, the clusters are not of equal size. Therefore, we use an extended version of Eq. (9.1) as a goal function for the control of an $M$-cluster state in a network of $N$ nodes:

$$
\begin{aligned}
& Q_{M}=\frac{1-f_{M}(\varphi)+\frac{N^{2}}{2}{ }_{p=1}^{M-1} f_{p}(\varphi)+\frac{1}{2}_{i, k=0}^{N-1}\left(r_{i}-r_{k}\right)^{2}}{q_{M}} \\
& +\frac{c}{2}{ }_{0}^{t N-1} \quad{ }_{k=0}^{N-1} G_{k i}\left(t^{\prime}\right)-1 \quad d t^{\prime} .
\end{aligned}
$$

$r_{j}$ and $\varphi_{j}, j=0, \ldots, N-1$, are the amplitude and the phase of the $j$ th oscillator, respectively. $\varphi$ is the vector $\varphi \equiv\left(\varphi_{0}, \ldots, \varphi_{N-1}\right)$ and $f_{M}(\varphi)$ the generalized Kuramoto order parameter given by

$$
f_{M}(\varphi)=\frac{1}{N^{2}}{ }_{j=0}^{N-1} e^{\mathrm{i} M \varphi_{j}}{ }_{k=0}^{N-1} e^{-\mathrm{i} M \varphi_{k}} ;
$$

see also Secs. 3.3 and 9.2. Recall that the sum over $p$ represents a penalty term. In comparison to Eq. (9.1), the condition $p \mid M$ (meaning that $p$ has to be a factor of $M$ ) was dropped in the sum, in other words penalty terms are added for all $p$-cluster states, where

\footnotetext{
${ }^{1}$ In the previous Chapters, I discussed that each $M$-state might, depending on $M$ and the number of nodes $N$, correspond to several different states with different $m$ where $m$ define the phase difference between neighboring nodes, i.e., $\varphi_{(i+1)} \bmod N-\varphi_{i}=2 \pi m / N$, and $M$ relates to $m$ according to $M=\operatorname{lcm}(m, N) / m$ (see Sec. 5.2). Recall that lcm stands for the least common multiplier. Here, the different $m$ states for the same $M$ cannot be distinguished any longer because the term "neighboring nodes" is not well defined in a topology which is not a regular ring network. Thus, in the following, I will only distinguish between different $M$ but not $m$.
} 
$p<M$. This ensures clusters of equal size since it prevents side maxima of the goal function. Synchronization of the radii is reached due to the term $i, k\left(r_{i}-r_{k}\right)^{2}$, where $r_{i}$ is the amplitude of the $i$ th oscillator.

The last term in Eq. (10.1), where $\mathbf{G}$ is the $N \times N$ coupling matrix and $c>0$ is a parameter, yields a unity row sum. Without it, a constant but arbitrary row sum would arise. Ensuring unity row sum helps to avoid side effects of changing the effective coupling strengths by this arbitrary row sum. As this term takes into account all deviations from the unity row sum during the growth of the network, $Q_{M}$ will not vanish completely in the goal state. Thus, $q_{M}$ is a better measure for the quality of synchronization. Although this might be regarded as a disadvantage of the integral in the unity-row-sum term, the advantage of the integral is that only terms in $G_{i j}$ but not in $\dot{G}_{i j}$ appear in the right hand side of the SG algorithm. Thus, it is not necessary to solve for $\dot{G}_{i j}$.

\subsection{AdAPTING THE TOPOLOGY}

As in the previous Chapter, I consider as a model a network of delay-coupled StuartLandau oscillators (see Sec. 5.2), however, with a real-valued coupling strengths, i.e, here, the phase $\beta$ of the complex coupling strength $\sigma=K \exp (i \beta)$ is equal to zero. Then, the equation of motion for the phases and amplitudes reads

$$
\begin{aligned}
\dot{r}_{j}(t) & =\lambda-r_{j}^{2} r_{j}+K{ }_{n=0}^{N-1} G_{j n} r_{n, \tau} \cos \varphi_{n, \tau}-\varphi_{j}-r_{j}, \\
\dot{\varphi}_{j}(t) & =\omega+K{ }_{n=0}^{N-1} G_{j n} \frac{r_{n, \tau}}{r_{j}} \sin \varphi_{n, \tau}-\varphi_{j}
\end{aligned}
$$

with $r_{j}=\left|z_{j}\right|$ and $\varphi_{j}=\arg \left(z_{j}\right)$, where $z_{j}(t)$ is the state of the $j$ th node at time $t$. Recall that $\tau$ is the delay time, and $\omega$ and $\lambda$ are the frequency and the bifurcation parameter of an uncoupled node, respectively; for details on the Stuart-Landau oscillator see Sec. 5.2. We abbreviate delayed variables with a lower index $\tau$, for example, $r_{n}(t-\tau) \equiv r_{n, \tau}$.

In the following, an adaption algorithm for $\mathbf{G}$ will be derived ensuring synchronization in an $M$-cluster state. Recall the SG algorithm in its finite form (see also Eq. (7.4)):

$$
\frac{d \mathbf{u}}{d t}=-\gamma \nabla_{\mathbf{u}} \omega(\mathbf{z}, \mathbf{u}, t)
$$


where $\gamma=\gamma^{\mathrm{T}}>0$ is a positive definite gain matrix, $\mathbf{u}$ the control variable and $\omega(\mathbf{z}, \mathbf{u}, t)$ the speed at which the goal function $Q(\mathbf{z}(t), t)$ is changing, i.e., $\omega(\mathbf{z}, \mathbf{u}, t)=\dot{Q}=\frac{\partial Q(\mathbf{z}, t)}{\partial t}+$ $\left[\nabla_{\mathbf{z}} Q(\mathbf{z}, t)\right]^{\mathrm{T}} \mathbf{F}(\mathbf{z}, \mathbf{u}, t)$. For a recapitulation on the SG algorithm see Sec. 7.1.

The SG algorithm (10.4) with $u=\left(G_{00}, G_{01}, \ldots, G_{N-1, N-2}, G_{N-1, N-1}\right) \in \mathbb{R}^{N^{2}}$ and $\gamma_{i j}=\delta_{i j} \gamma_{G}$, where $\gamma_{G}$ is a positive constant, yields

$$
\dot{G}_{j n}=-\gamma_{G} \frac{\partial}{\partial G_{j n}} \dot{Q}_{M}=-\gamma_{G}{\frac{\partial}{\partial G_{j n}}}_{k=0}^{N-1} \frac{\partial q_{M}}{\partial \varphi_{k}} \frac{d \varphi_{k}}{d t}+\frac{c}{2}{ }_{i=0}^{N-1} G_{k i}-1
$$

where we use $Q_{M}$ as given by Eq. (10.1) as a goal function. Recall that $q_{M}$ is the reduced goal function, i.e., the goal function without the unity-row-sum term (see also Eq. (10.1)).

Finally, with Eqs. (10.1), (10.2), and (10.3) the following control scheme is obtained

$$
\begin{aligned}
\dot{G}_{j n}(t)= & -\gamma_{G} K \frac{r_{n, \tau}}{r_{j}} \sin \left(\varphi_{n, \tau}-\varphi_{j}\right) \\
& \times{ }_{k=0}^{N-1} \quad{ }_{p=1}^{M-1} p \sin \left[p\left(\varphi_{k}-\varphi_{j}\right)\right]-\frac{2 M}{N^{2}} \sin \left[M\left(\varphi_{k}-\varphi_{j}\right)\right] \\
& -2 \gamma_{G} K{ }_{k=0}^{N-1}\left(r_{j}-r_{k}\right) \quad r_{n, \tau} \cos \left(\varphi_{n, \tau}-\varphi_{j}\right)-r_{j}-\gamma_{G} c{ }_{i=0}^{N-1} G_{j i}-1 .
\end{aligned}
$$

Figure 10.2 shows an example of the evolution of the network by applying the control algorithm (10.6) with $Q_{8}$, i.e., the goal to reach an 8-cluster state. The initial topology is a unidirectional ring, see Fig. 10.2(a). However, the nodes are approximately depicted according to their position $z_{j}$ in the phase space; because the phase differences are initially random, the unidirectional coupling structure does not clearly show here. At $t=0$ the control is switched on (Fig. 10.2(b)), and links rapidly change as can be seen in Fig. 10.2(c). The final state of the network is shown Fig. 10.2(d): 8 equally sized clusters are formed (to distinguish all nodes, the nodes in one cluster are not depicted exactly according to their phases but on a circle around the point which would correspond to their phase and radius). Black links mark excitatory links, i.e., links that correspond to positive entries of the coupling matrix, while the blue links are inhibitory ones, i.e., links due to negative entries of the coupling matrix. Clearly, the final topology is characterized by a distinct distribution of excitatory and inhibitory links: While the inhibitory links mainly connect neighboring clusters, the excitatory ones dominate the connections to 


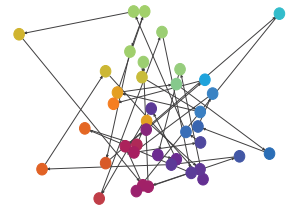

(a) $t=-50$ (initial state)

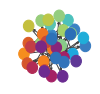

(b) $t=0$ (control starts)
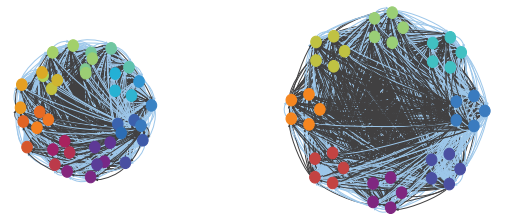

(c) $t=2$ (shortly after (d) $t=50$ (final state) control started)

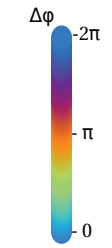

excitatory link inhibitory link

Figure 10.2.: Evolution of the network topology with the goal to achieve an 8-cluster state. Black: excitatory weighted links; blue: inhibitory weighted links. Node colors denote phase differences with respect to the first node. Parameters: $\lambda=0.1, \omega=1$, $c=0.01, K=0.1, \tau=\pi, \gamma_{G}=10, N=40, M=8$. Figure from [Lehnert et al., 2014].
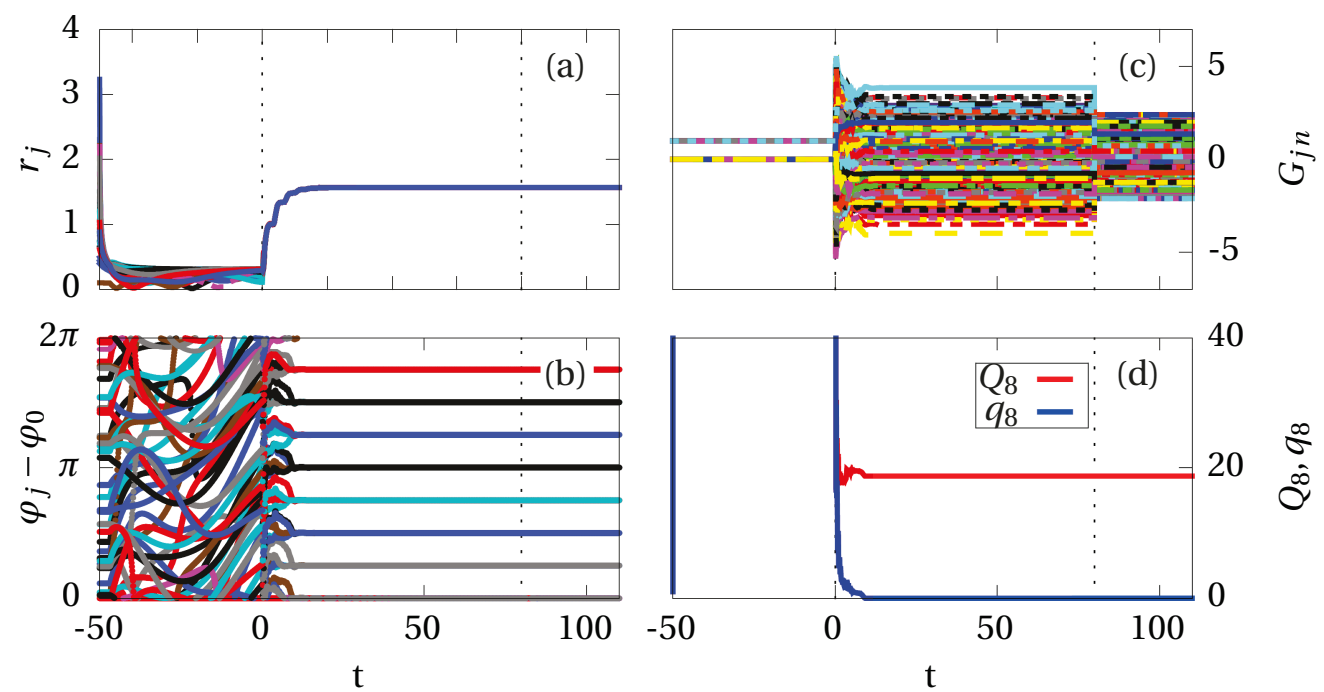

Figure 10.3.: Control of an 8-cluster state: (a) radii $r_{j}$, (b) phase difference $\varphi_{j}-\varphi_{0}$ with respect to the first node, (c) coupling weights $G_{i j}$, and (d) goal function $Q_{8}$ and its reduced part $q_{8}$ (excluding the unity-row-sum term). Control starts at $t=0$. The vertical dotted line at $t=80$ is explained in Sec. 10.6.2. Parameters as in Fig. 10.2. Figure from [Lehnert et al., 2014]. 


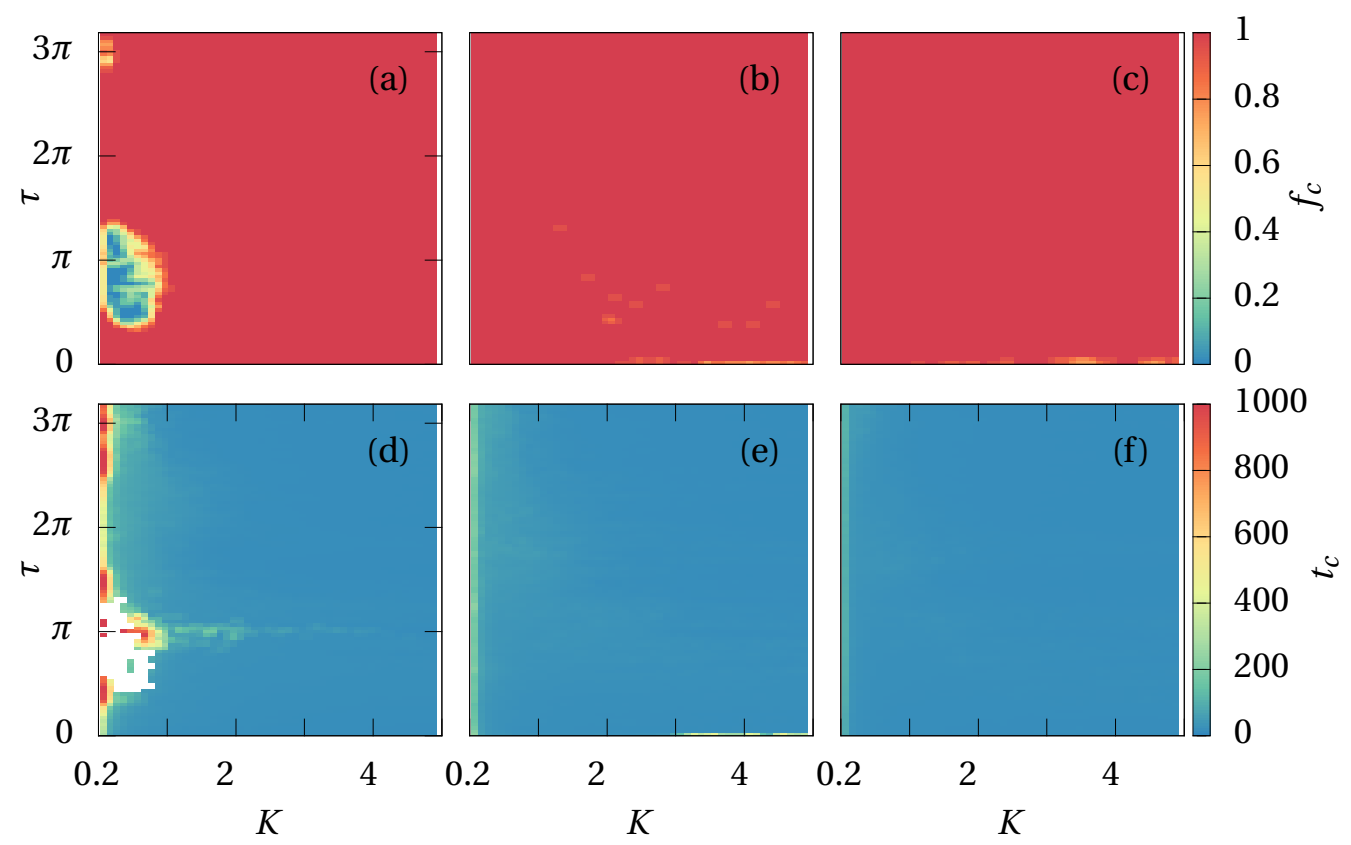

Figure 10.4.: Fraction $f_{c}$ of successfully controlled networks and control time $t_{c}$ as a function of coupling strength $K$ and delay $\tau$ for $(a),(d)$ zero-lag synchronization $(M=1)$, (b), (e) a 3-cluster state $(M=3)$, and $(d),(f)$ a splay-states $(M=15)$, respectively. White areas in (d) correspond to parameter values where the control is unsuccessful for all realizations. Averaged over 20 realizations. $N=15, \gamma_{G}=1$. Other parameters as in Fig. 10.2.

clusters further away in phase space. In the Sec 10.6 this distribution is investigated more closely.

Corresponding to the network realization in Fig. 10.2, Fig. 10.3 shows the time series of (a) the radii, (b) the phase differences, (c) the coupling weights, and (d) the goal function. After the control is switched on (dotted line at $t=0$ ), the radii and phases rapidly converge to the 8-cluster state, and the goal function $Q_{8}$ and its reduced part $q_{8}$ approach their minimum. The final common value of the radii $r_{i}, i=0, \ldots, N-1$, is determined by the value of the feedback gain $\gamma_{G}$ as will be discussed in Sec. 10.6. 


\subsection{ROBUSTNESS}

This Section discusses the performance of the method; in particular, the robustness towards different initial conditions, the control parameter dependency, and the time needed to reach the control goal are investigated. These issues are of particular interest as they determine the applicability of the method and allow for comparing the adaptive control of the topology with the control of the phase $\beta$ of the complex coupling strength presented in the previous Chapter.

Figure 10.4 depicts the fraction $f_{c}$ of successful realization and the time $t_{c}$ to reach the control goal for (a),(d) zero-lag synchronization, (b),(e) a 3-cluster state, and (d),(f) a splay-states, respectively, in dependence on the coupling strength $K$ and the delay time $\tau$. A realization is considered to be successful at time $t_{c}$ if $q_{M}<0.001$ for $t \in\left(t_{c}-1, t_{c}\right)$ and there has been no other time interval of length one before for which $q_{M}<0.001$ also holds. For each value of $K$ and $\tau$, the initial conditions for $z_{j}(t), j=0, \ldots, N-1$, are drawn from the complex interval $[-1,1] \times[-1,1]$. For the 3 -cluster state and the splay state, the method works remarkably fast and well for all value of $K$ and $\tau$ as becomes obvious from the success rate of $100 \%$ shown in panels (a), (b) and (c) and the low values of $t_{c}$ visible in panels (d), (e) and (f). Only in the case of zero-lag synchronization and $\tau=\pi$ the control needs more time and fails for small coupling strengths $K$. The reason is that at these parameters amplitude death sets in, which we do not consider as successful synchronization in a state of zero-lag synchrony. Comparing Fig. 10.4 with Fig. 9.6, which depicts $f_{c}$ and $t_{c}$ for the adaptive control of $\beta$, it becomes clear that the adaptive control of the topology is much more reliable and faster, in particular, for high coupling strength and delay times, where the adaptive control of $\beta$ fails. This suggests that the choice of appropriate parameters to adapt is crucial for the success of the SG-method in the control of dynamics on networks.

\subsection{LINEAR STABILITY ANALYSIS}

As in the case of the focus with adaptive time-delayed feedback control (see Sec. 8.2) and the adaptive control of the phase $\beta$ of the complex coupling strength (see Chapter 9), a linear stability analysis yields further insight into the problem. In the following, we label 
the nodes such that the final, synchronized state is described by

$$
\begin{aligned}
r_{j} & \equiv r_{0, M}, \\
\varphi_{j} & \equiv \Omega_{M} t+j \frac{2 \pi}{M}, \\
G_{i j} & \equiv G_{i j}^{*},
\end{aligned}
$$

for $j=0, \ldots, N-1$, where $r_{0, M}$ and $\Omega_{M}$ denote the common radius and the common frequency in an $M$-cluster state, respectively. $\mathbf{G}^{*}$ is the topology after successful control.

A variational equation is obtained by using the ansatz $r_{j}(t)=r_{0, M}\left[1+\delta r_{j}(t)\right], \varphi_{j}(t)=$ $\Omega_{M} t+j \frac{2 \pi m}{N}+\delta \varphi_{j}(t)$ and $G_{i j}(t)=G_{i j}^{*}+\delta G_{i j}(t)$. Substituting this ansatz into Eqs. (10.3) and (10.6) yields for the linear order of $\delta r_{j}, \delta \varphi_{j}$ and $\delta G_{i j}$ :

$$
\begin{aligned}
\delta \dot{r}_{j}= & -2 r_{0, M}^{2} \delta r_{j}+K_{n} G_{j n}^{*}-\cos \Phi_{1, n, j} \delta r_{j}+\sin \Phi_{1, n, j} \delta \varphi_{j} \\
& +K{ }_{n} G_{j n}^{*} \cos \Phi_{1, n, j} \delta r_{n, \tau}-\sin \Phi_{1, n, j} \delta \varphi_{n, \tau}+K_{n} \cos \Phi_{1, n, j}-1 \delta G_{j n}, \\
\delta \dot{\varphi}_{j}= & -K_{n} G_{j n}^{*} \sin \Phi_{1, n, j} \delta r_{j}+\cos \Phi_{1, n, j} \delta \varphi_{j} \\
& +K{ }_{n} G_{j n}^{*} \sin \Phi_{1, n, j} \delta r_{n, \tau}+\cos \Phi_{1, n, j} \delta \varphi_{n, \tau}+K_{n} \sin \Phi_{1, n, j} \delta G_{j n}, \\
\delta \dot{G}_{j n}= & -\gamma_{G} K \sin \Phi_{1, n, j}{ }_{k} \quad p^{2} \cos \frac{2 \pi p}{M}(k-j)-\frac{2 M^{2}}{N^{2}} \delta \varphi_{k}-\delta \varphi_{j} \\
& -2 \gamma_{G} K r_{0, M}^{2} \underset{k}{\left(\delta r_{j}-\delta r_{k}\right) \cos \Phi_{1, n, j}-1-\gamma_{G} c_{i} \delta G_{j i},}
\end{aligned}
$$

where we used the abbreviation $\Phi_{l, k, j}=l(k-j) 2 \pi / M-\Omega_{M} \tau$. From $\delta \dot{G}_{j n} \neq 0$ follows that no shift invariance in the direction of the adapted parameter exists as observed in the case of the adaptive control of a focus (cf. Chapter 7) or the adaptive control of the coupling phase $\beta$ (cf. Chapter 9). In Chapter 7 and Chapter 9, we observed that eigenvalues of the fixed point and the Floquet exponents of the limit cycle, respectively, of the system with adaptive control are given by the ones of the system without adaption plus an additional one equal to 0 , reflecting this invariance in the direction of the adapted parameter. Here, this is not longer the case but the Floquet exponents are completely changed by the adaptation. Consequently, the adaptation mechanism here is different from the previous Chapters. Instead of tuning the parameter under adaptive control to values for which the system with or without adaptation would be stable, the adaptive control introduces ad- 


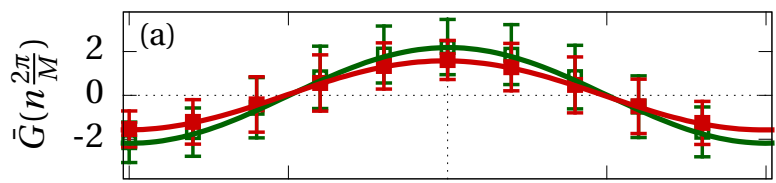

$$
\begin{gathered}
\tau=0 \text { 버 } \\
\tau=2 \pi \text { 배 }
\end{gathered}
$$

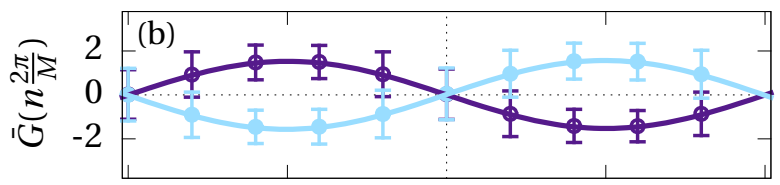

$$
\begin{gathered}
\tau=\frac{\pi}{2} \\
\tau=\frac{3 \pi}{2}
\end{gathered}
$$

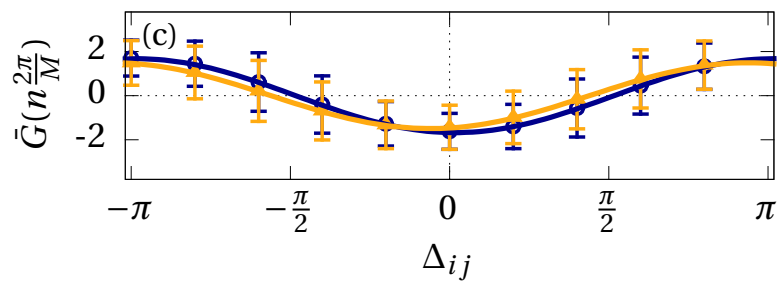

$\tau=\pi$ ю1

$\tau=3 \pi$

Figure 10.5.: Average link strength $\bar{G} \frac{2 \pi}{M} n$ versus phase differences $\Delta_{i j}=$ $\lim _{t \rightarrow \infty}\left[\varphi_{i}(t)-\varphi_{j}(t)\right]$ in the final state as defined in Eq. (10.9) for different values of $\tau$ : (a) $\tau=0$ and $\tau=2 \pi$, (b) $\tau=\pi / 2$ and $\tau=3 \pi / 2$, and (c) $\tau=\pi$ and $\tau=3 \pi$. $N=30, M=10, K=0.08$. Average over 100 realizations. Other parameters as in Fig. 10.2. Figure from [Lehnert et al., 2014].

ditional degrees of freedom which act stabilizingly.

\subsection{STRUCTURAL PROPERTIES}

In the field of complex networks, topological features enhancing or weakening synchronizability are of great interest [Pecora and Carroll, 1998; Chavez et al., 2005; Lehnert et al., 2011a; Keane et al., 2012]. Here, we discuss the structural properties of the networks after successful control, in order to elucidate the common features that enable synchronization in a cluster state. To this end we consider the coupling weights of the final topology as a function of the final phase difference, i.e., the function $\bar{G}_{i j}\left(\Delta_{i j}\right)$ where $\Delta_{i j} \equiv \lim _{t \rightarrow \infty}\left[\varphi_{i}(t)-\varphi_{j}(t)\right]$. In Fig. 10.5 we plot

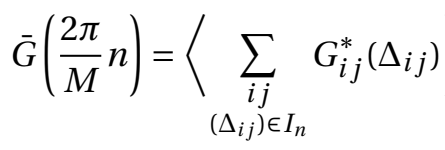




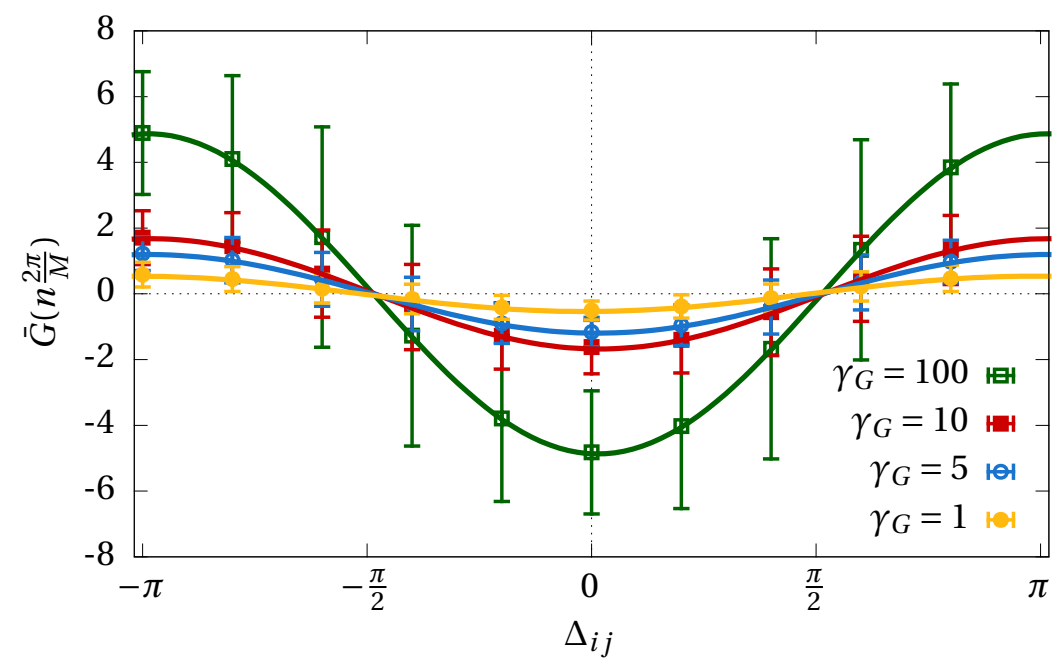

Figure 10.6.: Average link strength $\bar{G} \frac{2 \pi}{M} n$ versus phase differences $\Delta_{i j}=$ $\lim _{t \rightarrow \infty}\left[\varphi_{i}(t)-\varphi_{j}(t)\right]$ in the final state as defined in Eq. (10.9) for different values of $\gamma_{G}$ : green empty squares: $\gamma_{G}=100$; red filled squares: $\gamma_{G}=100$; blue empty circles: $\gamma_{G}=100$; yellow filled circles: $\gamma_{G}=100 . N=30, M=10, K=0.08$. Average over 100 realizations. Other parameters as in Fig. 10.2. 
with the interval $I_{n}=\frac{2 \pi n-\pi}{M}, \frac{2 \pi n+\pi}{M}, n=-M / 2, \ldots, M / 2-1$ if $M$ is even and $n=-(M-$ 1) $/ 2, \ldots,(M-1) / 2$ if $M$ is odd. $\langle\cdot\rangle$ denotes the ensemble average over 100 realizations, i.e., $\bar{G} \frac{2 \pi}{M} n$ is the average of all weights linking nodes which have a phase difference in the interval $I_{n}$. In the case of successful control these are the weights linking nodes in cluster $i$ with nodes in cluster $(i+n) \bmod M$. Figure 10.5 depicts the weights for different delay times. Obviously, the curves have the form of a delay time shifted cosine: i.e., $\bar{G} \frac{2 \pi}{M} n \propto$ $\cos \frac{2 \pi n}{M}-\tau$. This explains the topological structure we observe in Fig. 10.2(c): For $\tau=\pi$, we expect a structure as described by Fig. 10.5(c). Thus, a negative coupling between nodes with a small phase difference and a positive coupling between nodes with a phase equal or close to $\pi$.

Figure 10.6 shows $\bar{G} \frac{2 \pi}{M} n$ vs. $\Delta_{i j}$ for $\tau=\pi$ and different values of the feedback gain $\gamma_{G}$, where the green, red, blue, and yellow symbols correspond to $\gamma_{G}=100, \gamma_{G}=10$, $\gamma_{G}=5$, and $\gamma_{G}=1$, respectively. Clearly, the amplitude of the cosine depends on $\gamma_{G}$. In Subsecs. (10.6.1) and 10.6.2, it will be shown that this amplitude is proportional to the square of the common radius $r_{0, M^{2}}$. In conclusion, the common radius depends on the feedback gain where higher feedback gains results in higher radii.

\subsubsection{Existence of cluster solutions}

Insight into the network structure can be obtained by a row-wise discrete Fourier transform of the coupling matrix after successful control. To do so, we introduce the following $N \times M$ matrix

$$
\Gamma_{j k}={ }_{l=0}^{\bar{m}-1} G_{j, k+l M}^{*}
$$

where $\bar{m}$ is the number of nodes in one cluster, i.e, $\bar{m}=N / M$. Thus, $\Gamma_{j k}$ represents the total input which node $j$ receives from all nodes in cluster $k$. In the case of a splay state, i.e., $M=N, \Gamma$ and $\mathbf{G}$ coincide. We now represent each row of $\Gamma$ as a discrete Fourier series

$$
\begin{aligned}
\Gamma_{j k}= & \frac{b_{0}^{j}}{2 \cos \left(\Omega_{M} \tau\right)}+{ }_{l=1}^{N_{0}-1} a_{l}^{j} \sin \Phi_{l, k, j}+b_{l}^{j} \cos \Phi_{l, k, j} \\
& +\frac{b_{N_{0}}^{j}}{\frac{\cos \left(\Omega_{M} \tau\right)^{2}}{\text { if } M \text { is even }}} \frac{\cos \Phi_{N_{0}, k, j}, \quad j=0, \ldots, N-1, k=0, \ldots, M-1,}{}
\end{aligned}
$$


with the abbreviation $\Phi_{l, k, j}=l(k-j) 2 \pi / M-\Omega_{M} \tau$, which was already used in Eq. (10.8). $N_{0}=(M+1) / 2$ if $M$ is odd and $N_{0}=M / 2$ if $M$ is even. The Fourier coefficients are given by $(j=0, \ldots, N-1)$

$$
\begin{array}{ll}
a_{l}^{j}=\frac{2}{M}{ }_{k=0}^{M-1} \Gamma_{j k} \sin \Phi_{l, k, j}=\frac{2}{N}{ }_{k=0}^{N-1} G_{j k}^{*} \sin \Phi_{l, k, j}, & l=0, \ldots, N_{0}-1, \\
b_{l}^{j}=\frac{2}{M}{ }_{k=0}^{M-1} \Gamma_{j k} \cos \Phi_{l, k, j}=\frac{2}{N}{ }_{k=0}^{N-1} G_{j k}^{*} \cos \Phi_{l, k, j}, & l=0, \ldots, N_{0} .
\end{array}
$$

With the unity-row-sum condition, i.e., $\quad{ }_{k=0}^{M-1} \Gamma_{j k}={ }_{k=0}^{N-1} G_{j k}=1, b_{0}^{j} \equiv b_{0}=2 \cos \left(\Omega_{M} \tau\right) / M$ follows. Note that $\bar{G} n \frac{2 \pi}{M}$ from Eq. (10.9) can be expressed as

$$
\bar{G} n \frac{2 \pi}{M}={ }_{j=0}^{N-1} \Gamma_{j,(j+n) \bmod M} .
$$

Next, we discuss the necessary conditions for $a_{l}^{j}$ and $b_{l}^{j}$ such that an $M$-cluster state exists as a solution of Eq. (10.3). Substituting Eq. (10.7) into Eq. (10.3) yields the following conditions for $r_{0, M}$ and $\Omega_{M}$ :

$$
\begin{aligned}
r_{0, M}^{2} & =\lambda+K{ }_{k=0}^{N-1} G_{j k}^{*} \cos \Phi_{1, k, j}-1, \\
\Omega_{M} & =\omega+K{ }_{k=0}^{N-1} G_{j k}^{*} \sin \Phi_{1, k, j} .
\end{aligned}
$$

Using Eq. (10.12) this can be rewritten as

$$
\begin{aligned}
r_{0, M}^{2} & =\lambda+K \frac{b_{1}^{j} N}{2}-1, \\
\Omega_{M} & =\omega+K \frac{a_{1}^{j} N}{2} .
\end{aligned}
$$

Equation (10.15) has to be fulfilled for all $j=0, \ldots, N-1$. Thus, only if $a_{1}^{0}=a_{1}^{1}=\ldots=$ $a_{1}^{N-1} \equiv a$ and $b_{1}^{0}=b_{1}^{1}=\ldots=b_{1}^{N-1} \equiv b$ a solution with a common radius and a common frequency exists. Note that there is no restriction on the higher Fourier coefficients, i.e., 
on $a_{l}^{j}$ and $b_{l}^{j}$ with $l>1$.

10.6.2 Stability of cluster states

So far we have discussed the existence of the solution but not its stability. Unfortunately, it is not possible to carry out a systematic stability analysis in all Fourier coefficients as their number strongly increases for large $M$. However, there is evidence that the higher Fourier coefficients do not affect the stability. Rewriting the variational equation (10.8) with the help of the Fourier coefficients given by Eq. (10.12) yields

$$
\begin{aligned}
\delta \dot{r}_{j}= & \left(-3 r_{0, M}^{2}+\lambda-K\right) \delta r_{j}+\left(\Omega_{M}-\omega\right) \delta \varphi_{j}+K_{n} G_{j n}^{*} \cos \Phi_{1, n, j} \delta r_{n, \tau}-\sin \Phi_{1, n, j} \delta \varphi_{n, \tau} \\
& +\frac{K N}{2} \delta b_{1}^{j}-\frac{\delta b_{0}^{j}}{\cos \left(\Omega_{M} \tau\right)}, \\
\delta \dot{\varphi}_{j}= & \left(-\Omega_{M}+\omega\right) \delta r_{j}+\left(-r_{0, M}^{2}+\lambda-K\right) \delta \varphi_{j}+K_{n} G_{j n}^{*} \sin \Phi_{1, n, j} \delta r_{n, \tau}+\cos \Phi_{1, n, j} \delta \varphi_{n, \tau} \\
& +\frac{K N}{2} \delta a_{1}^{j}, \\
\delta \dot{a}_{l}^{j}= & -\delta_{l, 1} \gamma_{G} K{ }_{k} \quad p^{2} \cos \frac{2 \pi p}{M}(k-j)-\frac{2 M^{2}}{N^{2}}\left(\delta \varphi_{k}-\delta \varphi_{j}\right), \\
\delta \dot{b}_{l}^{j}= & -\delta_{l, 0} \frac{1}{2} N^{2} \gamma_{G} c \delta b_{0}^{j}-2 \gamma_{G} K r_{0, M}^{2}{ }_{k}\left[\delta_{l 1}-2 \cos \left(\Omega_{M} \tau\right) \delta_{l 0}\right]\left(\delta r_{j}-\delta r_{k}\right) .
\end{aligned}
$$

It follows from Eq. (10.16) that $\delta \dot{a}_{l}^{j}=0$ and $\delta \dot{b}_{l}^{j}=0$ for $l>1$ because Eqs. (10.16c) and (10.16d) contain only terms which are proportional to $\delta_{l, 0}$ or $\delta_{l, 1}$. Thus, we have a shift invariance in the directions of the higher Fourier coefficients. In the following, $\delta a_{l}^{j}$ and $\delta b_{l}^{j}$ with $l>1$ are treated as constant.

With the abbreviations $\xi_{j} \equiv\left(\delta r_{j}, \delta \varphi_{j}, \delta b_{0}^{j}, a_{1}^{j}, b_{1}^{j}\right)$ and $\xi \equiv\left(\xi_{0}, \ldots, \xi_{N-1}\right)$, Eq. (10.16) can be rewritten in a compact form as

$$
\dot{\boldsymbol{\xi}}=\mathbb{1} \otimes D \mathbf{f} \boldsymbol{\xi}+K(\mathbf{B} \otimes \mathbf{H}+\mathbf{C} \otimes \mathbf{I}) \boldsymbol{\xi}_{\tau}+\mathbf{A} \otimes \mathbf{J} \boldsymbol{\xi}+\mathbf{D} \otimes \mathbf{K} \boldsymbol{\xi},
$$


where $\mathbb{1}$ is a $N \times N$ unity matrix. $D \mathbf{f}$ describes the local dynamics:

$$
\begin{array}{ccccc}
-3 r_{0, M}^{2}+\lambda-K & \Omega_{M}-\omega & -\frac{K N}{2 \cos \left(\Omega_{M} \tau\right)} & 0 & \frac{K N}{2} \\
-\Omega_{M}+\omega & -3 r_{0, M}^{2}+\lambda-K & 0 & \frac{K N}{2} & 0 \\
D \mathbf{f}=4 \gamma_{G} K r_{0, M}^{2} N \cos \left(\Omega_{M} \tau\right) & 0 & -\frac{N^{2} \gamma_{G} c}{2} & 0 & 0 \\
0 & -\frac{\gamma_{G} K 2 M^{2}}{N} & 0 & 0 & 0 \\
-2 \gamma_{G} K r_{0, M}^{2} N & 0 & 0 & 0 & 0
\end{array},
$$

where we used ${ }_{k} \cos 2 \pi p / M(k-j)=0$ because $2 \pi p / M \notin \mathbb{N}$.

$\mathbf{B}$ and $\mathbf{C}$ are given by $B_{i j}=G_{i j}^{*} \cos \left(\Phi_{1, n, j}\right)$ and $C_{i j}=G_{i j}^{*} \sin \left(\Phi_{1, n, j}\right), i, j=0, \ldots, N-1$, respectively. $\mathbf{B}$ and $\mathbf{C}$ transmit the delayed coupled terms. The term of the form ${ }_{k} \ldots \delta r_{k}$ in Eq. (10.16d) constitutes an all-to all coupling (see Sec. 2.3.1) which is described by the all-to-all matrix A with self-coupling, i.e., $A_{i j}=1, i, j=0, \ldots, N-1$. D takes care off the more complicated all-to-all coupling into the term $\delta \dot{a}_{l}^{j}$ and is given by $D_{i j}=$ $-\gamma_{G} K \quad p_{p}^{2} \cos \frac{2 \pi p}{M}(k-j)-\frac{2 M^{2}}{N^{2}}$.

$\mathbf{H}, \mathbf{I}, \mathbf{J}$ and $\mathbf{K}$ represent the coupling schemes: $\mathbf{H}$ is a matrix with $H_{00}=H_{00}=1$ and zeros otherwise. Analogously, $\mathbf{I}$ is a matrix with $I_{01}=-I_{10}=1$ and zeros otherwise. $\mathbf{K}$ is a matrix with $K_{31}=1$ and zeros otherwise, and $\mathbf{J}$ is given by $J_{2,0}=-4 \gamma_{G} K r_{0, M}^{2} \cos \left(\Omega_{M} \tau\right)$, $J_{4,0}=2 \gamma_{G} K r_{0, M}^{2}$, and zeros otherwise.

Unfortunately, a master stability approach (see Sec. 3.2) is not possible here since the matrices $\mathbf{A}, \mathbf{B}, \mathbf{C}$, and $\mathbf{D}$ do not commute. However, commuting matrices are a prerequisite for the application of the master stability approach in cases where more than one coupling matrix is present; see Sec. 5.1.5 or [Dahms, 2011; Dahms et al., 2012]. Furthermore, a stability analysis in dependence of all higher Fourier coefficients is not possible since their number grows linearly in $N$ and $M$. However, their effect can be tested by choosing random coefficients.

The Floquet exponent $\Lambda$ that arises from Eq. (10.17) can be obtained from the corresponding characteristic equation

$$
0=\operatorname{det} \mathbb{1} \otimes D \mathbf{f}-\Lambda \mathbb{1}_{5 N}+K(\mathbf{B} \otimes \mathbf{H}+\mathbf{C} \otimes \mathbf{I}) \exp (-\Lambda \tau)+\mathbf{A} \otimes \mathbf{J}+\mathbf{D} \otimes \mathbf{K}
$$

where $\mathbb{1}_{5 N}$ is a unity matrix of dimension $5 N$; see also Appendix A for a recapitulation on solving linear delay differential equations. Equation (10.19) yields an infinite number of Floquet exponents for $\tau>0$. We consider only the one with the largest real part. If this 


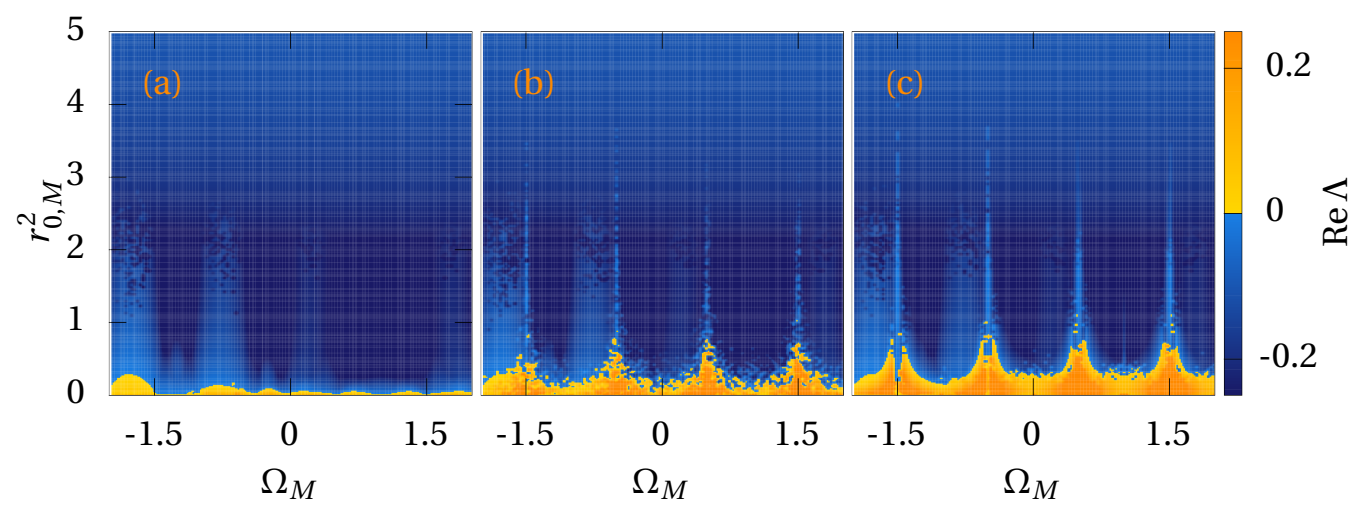

Figure 10.7.: Stability as a function of common frequency $\Omega_{M}$ and radius $r_{0, M}^{2}$ for (a) vanishing higher Fourier coefficients, i.e., $a_{l}^{j}=b_{l}^{j}=0$ for $l>1$, (b) random higher Fourier coefficients, (c) constant higher Fourier coefficients, i.e, $a_{l}^{j}=b_{l}^{j}=10$ for $l>1 . N=8$, $M=4$. Other parameters as in Fig. 10.2.

real part $\operatorname{Re} \Lambda$ is negative, the cluster solution given by Eq. (10.14) or (10.15) will be stable otherwise the solution is unstable.

Figure 10.7 shows the result of this stability analysis for (a) all higher Fourier coefficients being zero, i.e., $a_{l}^{j}=b_{l}^{j}=0$ for $l>1$ and $j=0, \ldots, N-1$, (b) random higher Fourier coefficients, and (c) constant higher Fourier coefficients, i.e, $a_{l}^{j}=b_{l}^{j}=10$ for $l>1$ and $j=0, \ldots, N-1$, in dependence on the common radius $r_{0, M}^{2}$ and the common frequency $\Omega_{M}$. Random means that for each value of $r_{0, M}^{2}$ and $\Omega_{M}$ the coefficients are drawn from a uniform distribution on the interval $[-10,10]$. Clearly it shows that the stability is only affected by the higher Fourier coefficients if $r_{0, M}^{2}$ is small: For small $r_{0, M}^{2}$ the unstable regions (yellow to orange color code) have a qualitatively different form in panels (a), (b) and (c), while for large $r_{0, M}^{2}$ stability is found in all three cases. This is due to the terms $\delta \dot{r}_{j}=-3 r_{0, M}^{2} \delta r_{j}$ and $\delta \dot{\varphi}_{j}=-r_{0, M}^{2} \delta \varphi_{j}$ in Eq. (10.16a) and Eq. (10.16b), respectively, which act stabilizing and outweigh the other terms for large $r_{0, M}^{2}$.

Another possibility to test the influence of the higher coefficients is to disturb them during or after the course of the adaptation process. Figures 10.8 and 10.9 show the time series of $a_{l}^{j}, j=0, \ldots, N-1, l=1, \ldots, N_{0}-1$, and $b_{l}^{j}, j=0, \ldots, N-1, l=1, \ldots, N_{0}$, respectively. The time series are from the simulation shown in Fig. 10.3. Clearly, $a_{1}^{j}$ and $b_{1}^{j}$ converge to the common values $a$ and $b$, respectively, after the control has been switched on at $t=0$ assuring the existence of a common frequency and radius according to Eq. (10.15). In 
contrast, the higher Fourier coefficients do not approach each other. At $t=80$, the higher Fourier coefficients are set to a random value in the interval $[-3,3]$. This interval was chosen for convenience of depiction. Qualitatively, the result is the same for much larger random numbers. As expected and apparent in Figs. 10.3 and 10.9, $a$ and $b$, and thus, according to Eq. (10.15), the common frequency and radius, do not change as a result of this perturbation. The higher Fourier coefficients stay at their new values, since there is no need for them to readjust. In conclusion, the existence and stability of the cluster solution does not depend on the higher Fourier coefficients. Thus, the final values of these higher coefficients follow from the random initial conditions and are therefore random themselves. As a consequence they average out, while, on average, the first Fourier coefficients dominate the topology. In fact, the average topology is mainly given by $b$ as $a$ and, for large $M, b_{0}=2 \cos \left(\Omega_{M} \tau\right) / M$ are typically small. Thus,

$$
\bar{G} n \frac{2 \pi}{M}={ }_{j=0}^{N-1} \Gamma_{j,(j+n) \bmod M} \approx b \cos \frac{2 \pi n}{M}-\Omega_{M} \tau
$$

explaining the cosine form of the curves shown in Fig. 10.5.

We now can also explain the relation between the common radius $r_{0, M}{ }^{2}$ and the feedback gain $\gamma_{G}$ : From Fig. 10.6, we have concluded that the amplitude of the curve $\bar{G} n \frac{2 \pi}{M}$ depends on $\gamma_{G}$ (for the description of the Figure, see p. 195). We now know that this amplitude is given by $b$ and that $b$, according to Eq. (10.15), is proportional to the common radius $r_{0, M^{2}}$. We, thus, can conclude that the common radius is a function of the feedback gain. A high feedback gain drives the system to high radii, while for a low gain the common radii are smaller.

From Eq. (10.20), it follows that the curve $\bar{G} n \frac{2 \pi}{M}$ is periodic in the delay time, which is also observable in Fig. 10.5 when comparing the different panels. This is closely related to [Yanchuk and Perlikowski, 2009] where it has been shown that systems with time delay generically have families of periodic solutions which are reappearing for infinitely many delay times. Simultaneously, with the return of a solution the structure reappears to realize this solution.

\subsection{CHOOSING A FREQUENCY}

The representation of the coupling matrix in a Fourier series as in Eq. (10.11) is particularly convenient if one wants to choose a common frequency via constructing an appro- 


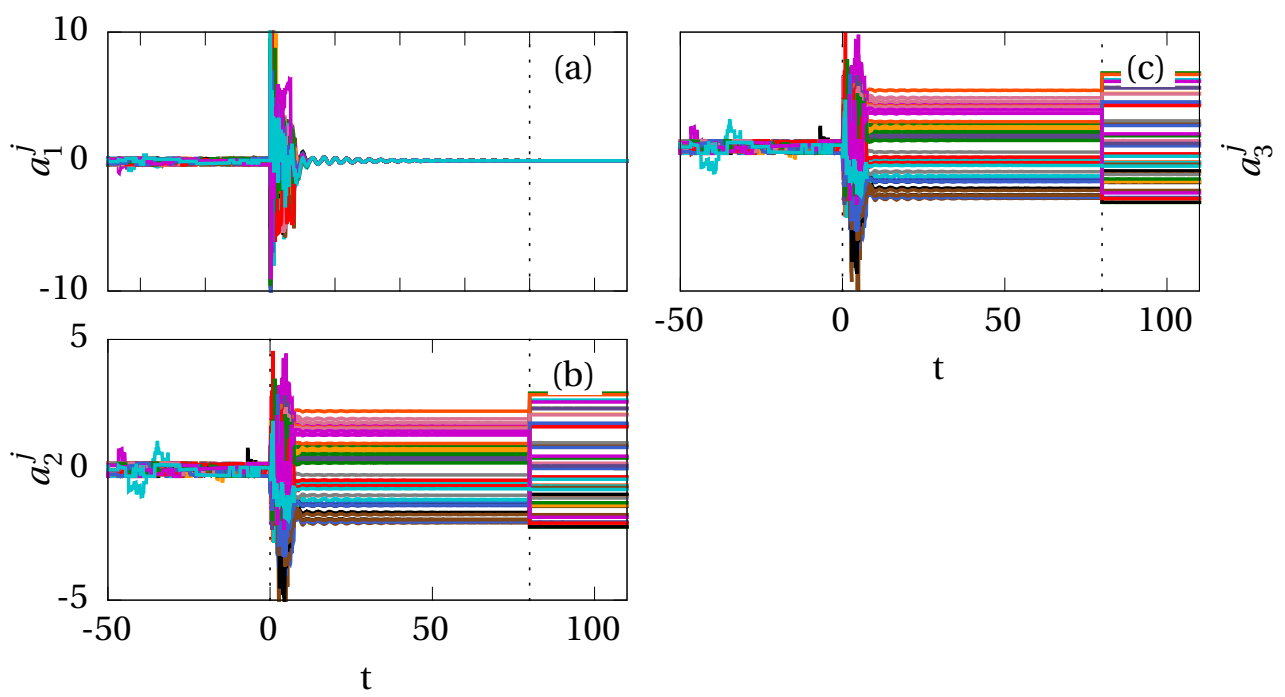

Figure 10.8.: Control of an 8-cluster state: Fourier coefficients as defined in Eq. (10.12): (a) $a_{1}^{j}$, (b) $a_{2}^{j}$, (c) $a_{3}^{j}$. At $t=0$, the control is switched on (first vertical dotted line). At $t=80, a_{2}^{j}$ and $a_{3}^{j}$ are set to random values (second vertical dotted line). Parameters as in Fig. 10.2. 

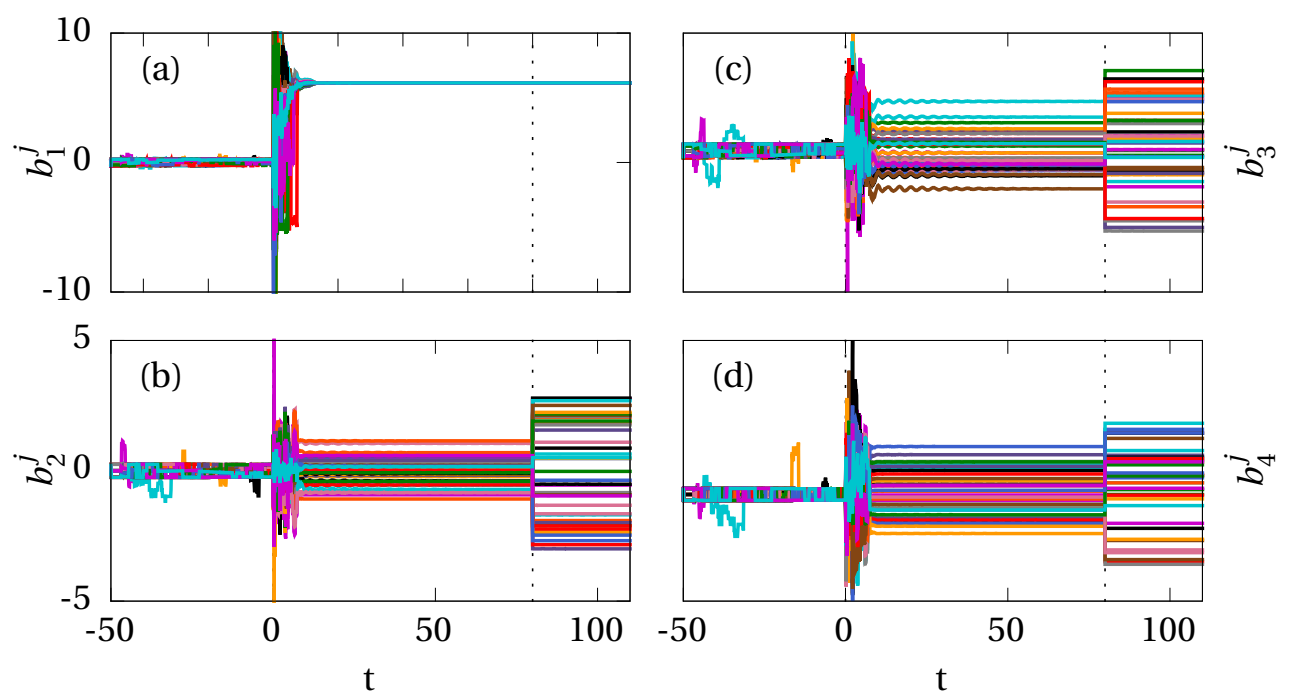

Figure 10.9.: Control of an 8-cluster state: Fourier coefficients as defined in Eq. (10.12): (a) $b_{1}^{j}$, (b) $b_{2}^{j}$, (c) $b_{3}^{j}$, (d) $b_{4}^{j}$. At $t=0$, the control is switched on (first vertical dotted line). At $t=80, b_{2}^{j}, b_{3}^{j}, b_{4}^{j}$ are set to random values (second vertical dotted line). Parameters as in Fig. 10.2. Figure from [Lehnert et al., 2014]. 

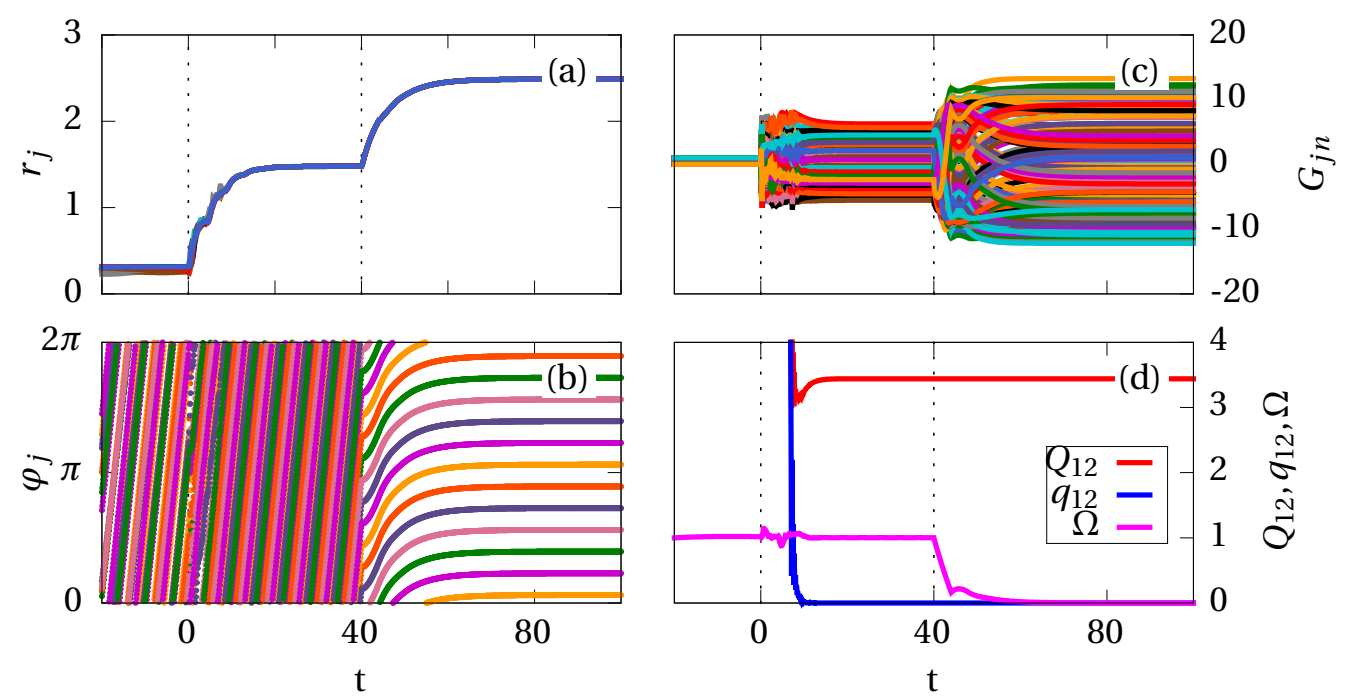

Figure 10.10.: Oscillation quenching of a splay state: (a) radii $r_{j}$, (b) phases $\varphi_{j}$, (c) coupling weights $G_{i j}$, and (d) goal function $Q_{12}$, its reduced part $q_{12}$ and the collective frequency $\Omega_{M} . N=12, M=12$. At $t=40$ the adaptive control is switched off and $a$ is set to $a=\frac{2 \omega}{N K}$. Other parameters as in Fig. 10.2. Figure from [Lehnert et al., 2014]. 
priate matrix. To select the common frequency $\Omega_{M}^{*}, a$ is set to

$$
a=\frac{2}{N} \frac{\Omega_{M}^{*}-\omega}{K}
$$

according to Eq. (10.15)(b). Note that we are free to choose the higher Fourier coefficients, i.e., $a_{l}^{j}$ and $b_{l}^{j}, l>1, j=0, \ldots, N-1$, when constructing $\Gamma$ according to Eq. (10.11). Furthermore, there is some freedom when choosing $\mathbf{G}$ from $\Gamma$ since Eq. (10.10) is only bijective if $M=N$, i.e., for the splay state.

As an example, Fig. 10.10 shows the quenching of oscillations in a splay state, i.e., we tune $\Omega_{M}^{*}$ to zero: At $t=0$ the adaptive control start with $M=N$, i.e., with the goal function leading to a splay state. At $t=40$ the adaptive control is switched off and $a$ is kept fixed at $a=\frac{2 \omega}{N K}$ forcing $\Omega_{M}$ to approach zero. As a result of $\Omega_{M}^{*}$ decaying to zero (see magenta line in Fig. $10.10(\mathrm{~d})$ ) while $a$ is fixed, the coupling weights slightly change after $t=40$ even though the adaptive control is switched off.

\subsection{CONTROL OF A SUBSET OF LINKS}

So far every link of the network was controlled. However, this is not necessary. It is sufficient to control a subset of links, while the other links are left fixed. This is useful for applications where not all links are accessible. We demonstrate this with an example of a directed random network constructed of $P$ links, which are chosen from the $L=N(N-1)$ possible links excluding self-coupling. From these $P$ links, we select, again randomly, $A$ links which are subject to adaptation as given by Eq. (10.6). As an example, Fig. 10.11 depicts the generation of a 3-cluster state in a network of 15 nodes. Here $30 \%$ of all links are accessible. Figure 10.11(a) shows the network before the control: Black links mark the $P-A$ fixed links, green links depict the $A$ links that will be adapted. One can see that only these links change their strength during the adaptation process as can be seen in Fig. 10.11(b), which displays the network after it has reached the desired 3-cluster state and the change of topology terminates.

The corresponding time series are shown in Fig. 10.12(a)-(d) for the radii, the phase differences, the elements of the coupling matrix, and the goal function, respectively. As can be seen in Fig. 10.12(b) and (d), after successful control with goal function $Q_{3}$ the network consists of 3 equally sized clusters and the reduced goal function $q_{3}$ is zero.

We now want to test how successful our method is in dependence upon the links present in the networks, and upon the fraction of these links subject to adaptation: Figure 10.13 

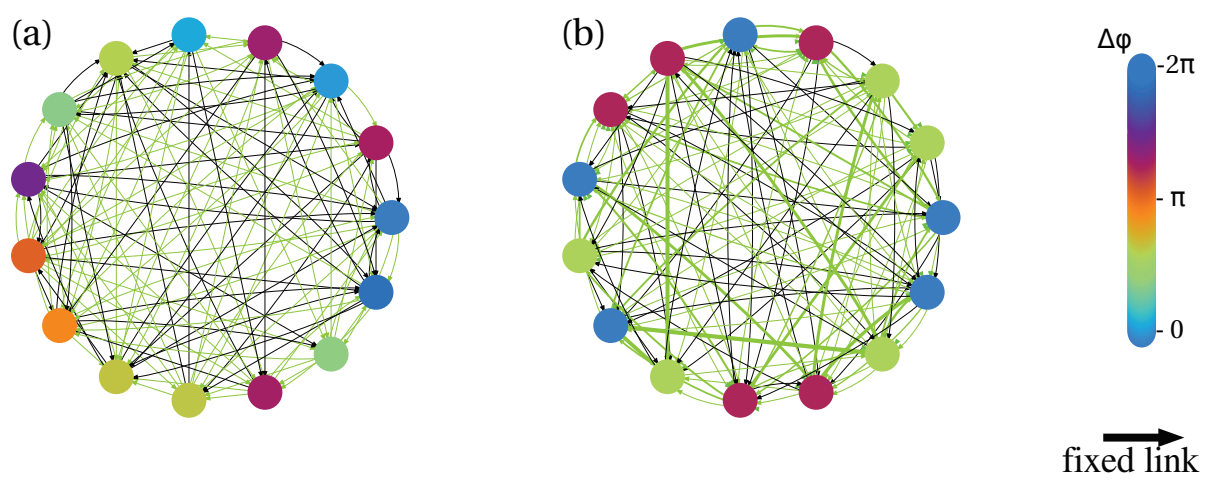

adapted link

Figure 10.11.: Topology (a) before $(t=0)$ and (b) after control, i.e., after the vanishing of all transient effects. Black links: fixed links; green: adapted links. Color code of nodes: Phase difference $\Delta \varphi=\varphi_{j}-\varphi_{0}$ with respect to the first node. Parameters: $\tau=3, A / L=$ $0.3, P / L=0.4, N=15, M=3$. Other parameters as in Fig. 10.2. Initial conditions: directed random network. Figure from [Lehnert et al., 2014].

shows the fraction $f_{c}$ of successfully controlled networks and as an inset, the control time $t_{c}$ as function of $P / L$ and $A / L$. We use the definition for successful control at time $t_{c}$ introduced in Sec. 10.4.

Note that the success rate is fairly independent of the total number of links $P$ in the network, but depends mainly on the ratio of adapted links to all possible links. In other words, the links additionally present in the network, but not subject to control, have almost no effect on the synchronizability. If the number of adapted links reaches about $40 \%$ the control still works in more than $90 \%$ of the cases.

A good approximation of the success rate $f_{c}$ can be obtained if we assume that for successful control each node in the networks needs at least two incoming links which are adapted. One adapted link is not sufficient because it could not change due to the unityrow sum condition. Only if a second incoming link is present the links can change in order to control the dynamics of the node because the effect of the adaptation of the first link on the row-sum can be counteracted by the second link. Figure 10.14 depicts $f_{c}$ vs. $A / L$ as red circles for a fixed ratio of (a) $P / L=0.4$, (b) $P / L=0.6$, and (c) $P / L=1$. The blue circles depict the fraction $p_{>1}$ of networks where all nodes have at least two incoming links. Obviously, $p_{>1}$ well approximates $f_{c}$ even though they are not identical meaning 

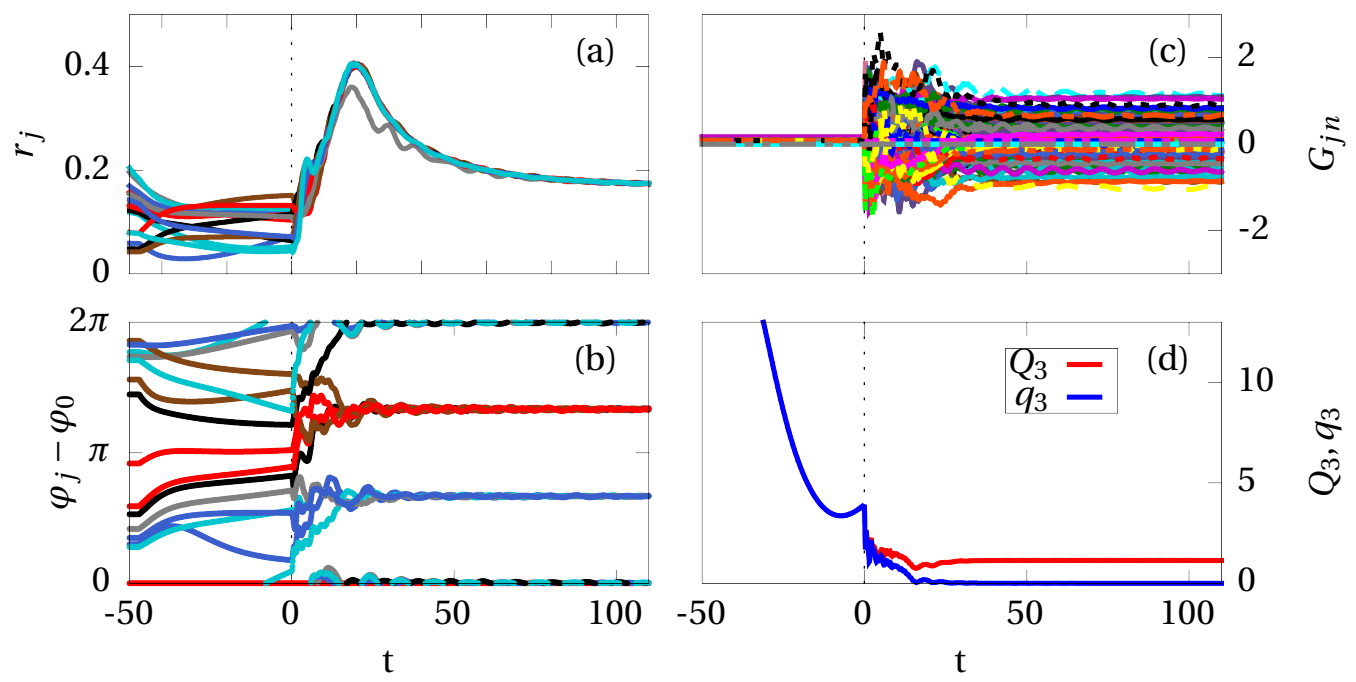

Figure 10.12.: Restricted control of a 3-cluster state: (a) radii $r_{j}$, (b) phase difference $\varphi_{j}-$ $\varphi_{0}$ with respect to the first node, (c) coupling weights $G_{i j}$, and (d) goal function $Q_{3}$ and it its reduced part $q_{3}$. Parameters as in Fig. 10.11. Figure from [Lehnert et al., 2014].
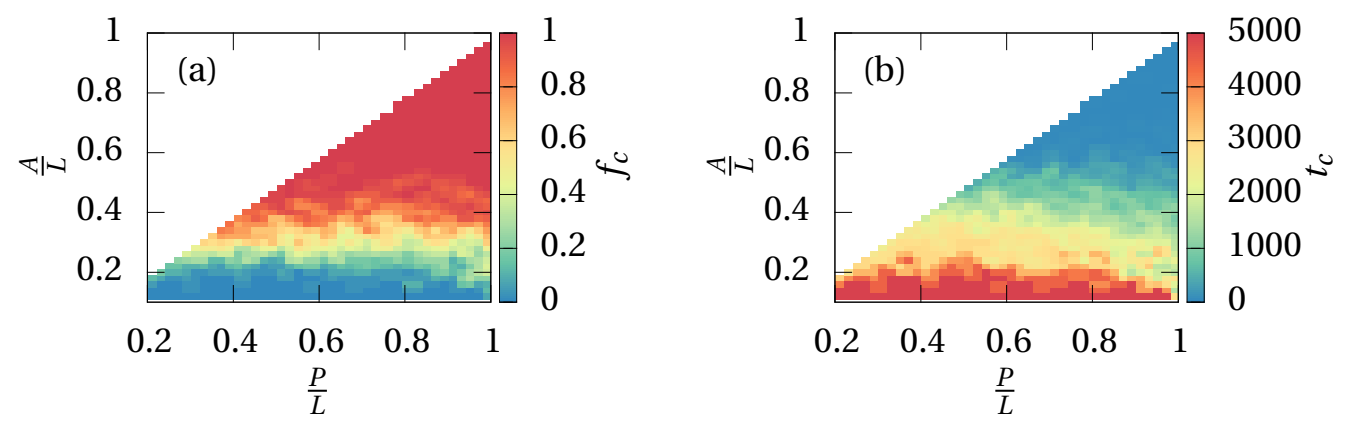

Figure 10.13.: Control of a subset of links. (a) Fraction $f_{c}$ of successfully controlled networks, and (b) time $t_{c}$ needed to reach the control goal as a function of the number of random links $P$ and the number of controlled links $A$, normalized by $L=N(N-1)$. 10 realizations. $K=0.2$. Other parameters as in Fig. 10.11. Figure modified from [Lehnert et al., 2014]. 


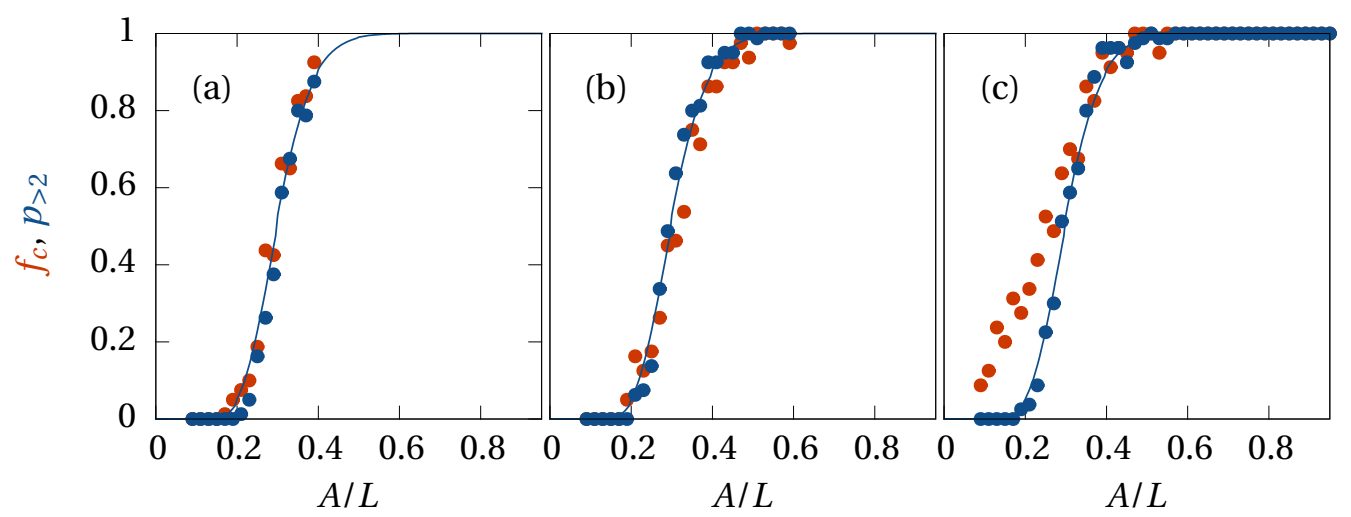

Figure 10.14.: Control of a subset of links for fixed ratio of (a) $P / L=0.4$, (b) $P / L=0.6$, and (c) $P / L=1$. Red circles: success rate $f_{c}$; blue circles: probability $p_{>1}$ that no node in the network has less than 2 incoming links which are adapted; blue line: $p_{>1}$ according to Eq. (10.22). $K=0.2 .80$ realizations for each value of $A / L$. Other parameters as in Fig. 10.11.

that cases exist where the network can be controlled though one node has less than two adapted incoming links, or where the control fails although each node has two incoming links.

The probability that no node in the network has less than two incoming links, i.e., the probability that all nodes have more than one incoming link, can be analytically calculated as

$$
p_{>1}=1-\left(p_{0} p_{1}+\bar{p}_{0} p_{1}+p_{0} \bar{p}_{1}\right),
$$

where $p_{i}, i=0,1$, is the probability that at least one of the nodes in the network has exactly $i$ links, and $\bar{p}_{i}$ is the complementary probability, i.e., $\bar{p}_{i}=1-p_{i}$. To calculate $p_{i}$, we assume $P / L=1$, i.e., a fully connected networks and distribute than the $A$ adapted links, meaning that we convert $A$ of the static links to adaptive links. The probability $p_{0}$ 
that at least one of the nodes has exactly zero incoming links reads

$$
p_{o}=1-1-\underbrace{A-1}_{\text {probability that none of the nodes has exactly zero incoming links }} \stackrel{N-\frac{N-1}{N(N-1)-i}}{N} .
$$

We calculate the probability that a particular node has zero incoming adapteds link by distributing the $A$ links on the $N(N-1)$ possible link positions, where we exclude the selffeedback positions. Since the considered node has $(N-1)$ possible incoming links the probability that it gets no link when the first link is distributed is

$$
1-\frac{N-1}{N(N-1)}
$$

reducing the number of possible links position to $N(N-1)-1$ because we do not allow double links. Thus, in the next step the probability that the link is not assigned to the node under consideration is given by

$$
1-\frac{N-1}{N(N-1)-1}
$$

This procedure continues until all links are allocated.

The probability $p_{1}$ that at least one of the nodes has exactly one incoming adapted link is given by

$$
\begin{aligned}
& p_{1}=1-1-{ }_{l=0}^{A-1} i_{i=0}^{l-1} 1-\frac{N-1}{N(N-1)-i)} \frac{N-1}{N(N-1)-l}{ }_{j=l+1}^{A-1} 1-\frac{N-2}{N(N-1)-j} \\
& \text { probability that a particular node has one incoming links } \\
& \text { probability that none of the nodes has exactly one incoming link }
\end{aligned}
$$

To calculate the probability that a particular node has exactly one incoming link, we assume that it acquires this link in the $l$ th step of the link distribution with a probability of $\frac{N-1}{N(N-1)-l}$. We, then, sum over $l$ to get all possible scenarios. In Fig. 10.14, $p_{>1}$ calculated according to Eq. (10.22) is depicted as a blue line as a function of the fraction of accessi- 
ble links $A$. Even though, we assumed in the derivation of Eq. (10.22) that $P / L=1, p_{>1}$ is reasonable well predicted by Eq. (10.22) even if $P / L<1$ as can bee seen in Fig. 10.14(a) and (b).

\subsection{CONCLUSION}

In adaptive networks, the topology changes according to the states of the nodes, while, in turn, the topology influences the node dynamics. Adaptive networks have been used to described all kind of systems that are characterized by a complicated interplay of the topology and the dynamics. Examples include neural networks, swarm networks, social networks, the food web, and networks in chemestry and biology. However, less effort has been put in the design of algorithms which shape the topology of a network such that the nodes are forced to a desired dynamical state.

The aim of this Chapter has been to make use of a changing topology to control cluster synchronization in a network of time-delay coupled oscillators. The method uses the speed-gradient (SG) algorithm to adapt the topology. The considered goal function is based on a generalized Kuramoto order parameter and is independent of the ordering of the nodes. An additional term ensures amplitude synchronization.

The control scheme is very robust. Numerically, we have studied the robustness towards different initial conditions and its dependence on the overall coupling strength and delay time. For all values of the delay time and coupling strength, the method works reliable and fast. Only for the case of zero-lag synchrony, we have found that the control fails for $\tau$ close to $\pi$ and small coupling strengths $K$ because amplitude death takes place. Thus, the method is able to overcome the shortcomings of the adaptive control of $\beta$ presented in Chapter 9, which is sensitive towards different initial conditions and needs an increasing amount of time for large $K$ and $\tau$.

A linear stability analysis reveals that the adaptive control does not work by changing the topology to a topology which will allows for stable cluster synchronization even if the adaptation process is switched off. Instead, additional degrees of freedom act in a stabilizing manner.

The topology of the network after successful control is modulated by the coupling delay. A row-wise discrete Fourier transform of the coupling matrix gives insight into these delay modulations. Necessary conditions for the existence of a common radius and a common frequency give rise to restrictions affecting the first Fourier coefficients, while there is no restriction for the higher Fourier coefficients. There is strong evidence that 
for sufficiently large common radii, the stability of the cluster states is not affected by the higher Fourier coefficients. In conclusion, the higher Fourier coefficients are mainly dependent on the random initial conditions and are therefore randomly distributed. On average, the network topology is therefore dominated by the first Fourier coefficients leading to the observed delay modulation.

We have found that the first Fourier coefficients can be used to find topologies that lead to cluster states with a given common frequency. As an example, we have quenched the oscillations in a Stuart-Landau oscillator. Such an inhomogeneous steady state is known as oscillation death [Koseska et al., 2010, 2013a,b; Zakharova et al., 2013] and has been found in various systems including tunnel diodes [Heinrich et al., 2010], neuronal networks [Curtu, 2010], and genetic oscillators [Koseska et al., 2009]. This example demonstrates that we have found a very versatile method to construct networks that show a desired dynamical behavior.

Since in many real-world networks not all links are accessible to control, the adaptation algorithm has been applied to random networks where a random subset of links has been subject to control, while the other links remained fixed. We have found that the control is successful if the number of adapted links is equal or higher than approximately $40 \%$ of all possible links, independently of the number of actual fixed links. For practical applications this opens up the possibility to apply the method more efficiently. 


\section{CONCLUSION}

\subsection{SUMMARY}

The focus of this thesis has been to study and to control the interplay of dynamics and topology in complex networks with time-delayed coupling where the dynamical patterns include zero-lag as well as group and cluster synchronization. In the first part, I have studied the stability of different synchronization patterns, while the second part has focused on the adaptive control of these dynamical states. Besides developing a theoretical framework, I have investigated neural networks as applications, where I have considered generic neural models like the FitzHugh-Nagumo or the SNIPER (saddle-node infinite period bifurcation) model as well as more sophisticated models like the adaptive exponential integrate-and-fire model.

The study of synchrony performed in part I of this thesis is based on the master stability function (MSF) which was suggested by Pecora and Carroll [1998] to investigate the stability of zero-lag synchronization in complex networks. I have discussed its application to neural networks consisting of excitable elements. Depending on the underlying bifurcation, type-I and type-II excitability can be distinguished. We have investigated these types on the example of the saddle-node infinite period (SNIPER) model and the FitzHugh-Nagumo system, which can be considered as generic models for the respective type of excitability. It has turned out that the rate of inhibition is a crucial parameter when controlling synchrony in these networks and that its effect depends upon the type of excitability and the delay time: In the FitzHugh-Nagumo model a transition from synchronization to desynchronization takes place as the number of inhibitory links approaches a critical value. While the same result applies to the case of the SNIPER model for large delays, the situation is much more complex for this model as the delays become small. Here, depending on the coupling and network parameters multiple transitions between synchronization and desynchronization can take place. 
The MSF can be generalized to group and cluster synchronization if the network topology is multipartite. This framework allows for incorporating different local dynamics in the different groups as well as different coupling strengths and delay times between the groups such that complex structural and dynamical patterns can be investigated. We have shown that for group synchrony the MSF as well as the coupling matrix are characterized by a discrete rotational symmetry which allows for restricting the evaluation of the MSF to a part of the complex plane. In the case of commuting coupling matrices, the limitation of multipartite networks can be lifted. In the case of two groups, for example, this allows for taking inter- as well as intra-group coupling into account. The emerging complexity has been demonstrated on the example of two groups of FitzHugh-Nagumo systems where we have chosen different delay times for the coupling between and the coupling inside the group. Depending on the ratio of delay times we have observed zerolag synchrony and antisynchronization as well as bursting dynamics.

The MSF for zero-lag as well as for group synchrony can be extended to non-smooth systems which are of particular interest for applications in neurosciences where integrateand-fire models are commonly used. These models are characterized by a discontinuity if the membrane voltage reaches a threshold. We have applied the MSF for non-smooth systems to networks of adaptive exponential integrate-and-fire model where we have considered a group of excitatory and a group of inhibitory neurons which we have modelled with different local dynamics. This is in contrast to previous studies which were either fully numerically and, thus, considered only example topologies, or used the MSF with identical dynamics for all nodes. Here, we have made use of the full potential of the MSF for group synchrony by employing different dynamics for excitatory and inhibitory neurons which is biologically more plausible. By these means, we have shown that for different parameter sets, and, thus, for different dynamical scenarios, a decreasing adaptation strength leads to destabilization of synchrony independently of the topology.

Part II of this thesis is devoted to the adaptive control of network dynamics. In adaptive methods one or several control parameters are constantly tuned. This is particularly useful in situations where parameters drift or are unknown since it allows for adapting the control to the changing environment automatically. In this thesis, I have employed the speed gradient method (SG) [Fradkov, 1979, 2007] to develop adaptive controllers to control unstable fixed points and unstable periodic orbits in uncoupled systems as well as states of cluster synchrony in delay-coupled network motifs and large networks. As models I have considered generic models, namely, the normal form of the unstable focus, the Rössler system which is a well known model for chaotic dynamics, and the Stuart-Landau oscillator which is paradigmatic for systems close to the Hopf bifurcation. 
I have investigated several adaptive controllers and discussed their robustness towards drifting parameters, noise, and different initial conditions. The controllers can cope with all of these challenges, however, not all perform equally well. Consider for example the control of cluster synchronization in networks of coupled Stuart-Landau oscillators, here it has been shown that due to the high multistability of the system without adaptive control, the tuning of one parameter does not suffice to realize a control fairly independently of the initial conditions for all different cluster states. Instead, in such a highdimensional system only the simultaneous adaptation of several control parameters allows for a control which is independent of different initial conditions in a large range of parameters. This was demonstrated on the example of the adaption of the topology, where each entry of the coupling matrix acts as a control parameter.

Two different stabilizing mechanism can be distinguished when considering the above controllers. In the first mechanism, the adaptive controller tunes the parameter to values for which the original system, i.e., the system without adaptive control is stable. The system then approaches the target state because the adaptive controllers of this first type do not change the stability of the original system. Instead, they only introduce one additional Lyapunov exponent equal to zero in the direction of the control parameter. In this case the adaptive control could be switched off once the goal state has been reached without this target state becoming unstable again. This is the mechanism behind the control of the unstable focus presented in Sec. 8.2 and the control of the coupling phase discussed in Chapter 9. In the second case, the control induces additional degrees of freedom meaning that a linear stability analysis of the combined system, i.e., the original system and the adaptive controller, contains several terms which stabilize the formerly unstable directions. Consequently, the target state can become unstable again if the adaptation is switched off. As an example for a control scheme relying on this mechanism consider the adaption of the topology investigated in Chapter 10 .

In summary, I have investigated the control of different states of synchrony. In the first part of the thesis, I have used the MSF and its generalizations to consider the dependency of synchronization on the system and coupling parameters as well as on the topology. This knowledge can be used to realize a control goal by choosing appropriate parameters or topologies. In the second part, I have employed adaptive control methods to find these appropriate parameters and topologies in a self-organized way without the need to know the details of the local dynamics. 


\subsection{OUTLOOK}

The topics addressed in the first part of this thesis, i.e., the MSF, its generalizations, and its application in neural networks with inhibition and delay, are already quite far developed, where this thesis has contributed substantially. The MSF has been generalized to cluster and group synchrony, non-smooth systems, and distributed delays. Different versions of the topology like network topologies with inhibitory and excitatory nodes rather than inhibitory and excitatory links have been considered (see references in Chapters 4 and 5). Therefore, a fundamental understanding of the interplay between the topology, synchrony, and inhibition could be reached in the studies of generic models like the SNIPER, the FitzHugh-Nagumo, or the Morris-Lecar model. The focus of further research should, therefore, be to consider more realistic neural models and to search for direct applications of the theory in neurophysiological questions.

In contrast, I believe that there exists a variety of possibilities to extent the work on adaptive controllers on a fundamental level. First of all, further studies of the robustness could be carried out regarding the control via adaptation of the topology. In this context, the following questions could be fruitful: What are the effects of delay distributions in this control method? Is the method able to cope with these heterogeneities? Which is the effect on the final topology? It could be expected that the simple cosine we observed in Fig. 10.5 depicting the final coupling weights vs. the final coupling strengths changes to a more complicated function to counteract the influence of the delay heterogeneities. Similarly, it would be interesting to investigate the robustness in the presence of uni- or bimodal distributions of the nodes' eigenfrequencies. A question would be whether the nodes arrange themselves during the course of control into groups in which the nodes resemble each other in frequency, i.e., whether the width of the frequency distribution within a group is smaller than the width of the overall distribution. A first step in the direction of heterogeneous parameters has been made in [Plotnikov et al., 2015], where we have investigated heterogeneous threshold parameters in networks of coupled FitzHugh-Nagumo systems and showed that an adaptive control of zero-lag synchrony is feasible.

Secondly, a comparison or combination with other control concepts would be worthwhile. For example, one could think of minimizing the wiring length in the adaptive control of topology by complementing the method with optimal control theory [Lewis et al., 2012]. In [Kaluza and Mikhailov, 2014] an adaptive control scheme involving timedelayed feedback has been developed and applied to control zero-lag synchronization in oscillatory networks by changing the link strength. In principal, this method could also be applied in the control of cluster states and a comparison to the results obtained in 
Chapter 10 would be interesting. In [Yanchuk et al., 2011; Popovych et al., 2011], it was shown that arbitrary periodic firing patterns can be achieved by appropriately choosing the delay times in feed-forward oscillatory neural loops. By using adaptive techniques similar to the ones discussed in Chapters 8-10, these delay times could be found automatically and, thus, an adaptive scheme to control theses patterns could be realized.

Furthermore, the adaptive control of other dynamical states in networks could be rewarding. Travelling waves have recently been investigated on networks with a certain degree of regularity, e.g., regular rings, tree networks, and small-world networks [Isele, 2014; Kouvaris et al., 2014; Isele and Schöll, 2015; Isele et al., 2015]; Turing patterns, which have been originally described in reaction diffusion systems, have been generalized to networks [Nakao and Mikhailov, 2010; Hata et al., 2012; Wolfrum, 2012; Hata et al., 2014]; Chimera states, where the nodes in a regular network with homogeneous local dynamics separate into two groups with distinctly different dynamical behavior, gained a lot of attention recently [Kuramoto and Battogtokh, 2002; Abrams and Strogatz, 2004; Sethia et al., 2008; Wolfrum and Omel'chenko, 2011; Wolfrum et al., 2011; Omelchenko et al., 2011, 2012; Hagerstrom et al., 2012; Omelchenko et al., 2013; Vüllings et al., 2014; Zakharova et al., 2014; Omelchenko et al., 2015]. In principle, all of these dynamical states could be controlled with an adaptive control scheme based on the SG method. However, Turing patterns on networks as well as Chimera states are characterized by a certain degree of randomness in their respective dynamical patterns. Thus, the challenge is to find a goal function which describes these dynamical patterns sufficiently well without requiring too much knowledge of the system. 


\section{A $\mid$ Delay Differential equations}

Linear delay differential equations (DDE) are (semi-)analytically solvable [Asl and Ulsoy, 2003]. Consider the following linear system of DDEs

$$
\dot{\mathbf{x}}(t)=\mathbf{A} \mathbf{x}(t)+\mathbf{B x}(t-\tau),
$$

with $\mathbf{x} \in \mathbb{R}^{d}$ and $\mathbf{A}$ and $\mathbf{B}$ being $d \times d$ matrices. As in the case of an ordinary linear equation we proceed with an exponential ansatz $\delta \mathbf{x}(t)=\mathbf{u} e^{\Lambda t}$ where $\mathbf{u}$ and $\Lambda$ are time independent. Inserting this Ansatz into Eq. (A.1) yields

$$
\Lambda \mathbf{u} e^{\Lambda t}=\mathbf{A}+\mathbf{B} e^{-\Lambda \tau} \mathbf{u} e^{\Lambda t} .
$$

Equation (A.2) has a solution only for $\mathbf{u}$ if the following transcendental equation is fullfilled

$$
\operatorname{det} \mathbf{A}+\mathbf{B} e^{-\Lambda \tau}-\Lambda \mathbb{1}_{d}=0,
$$

where $\mathbb{1}_{d}$ is the $d$-dimensional unity matrix. We refer to Eq. (A.3) as the characteristic equation of system (A.1). Equation (A.3) has an infinite number of roots $\Lambda_{1}, \Lambda_{2}, \ldots$. The solution of Eq. (A.1) is then given by a superposition of all modes:

$$
\mathbf{x}(t)={ }_{j=1}^{\infty} \mathbf{u}_{j} e^{\Lambda_{j} t} .
$$

However, if we are interested only in the stability of the origin of Eq. (A.1), for example, if we consider a variational equation and $\mathbf{x}$ corresponds to a variation, it suffices to consider the exponent $\Lambda_{i}$ with the largest real part. If this real part is negative the 
corresponding mode in Eq. (A.4) will decrease. Thus, the origin is stable. Otherwise, if the real part is positive, the mode will increase and the origin is unstable. For a variational equation, this would correspond to a decreasing or increasing variation, respectively.

For details on the spectrum $\Lambda_{1}, \Lambda_{2}, \ldots$ for large delay $\tau$ see [Lichtner et al., 2011]. 


\section{B | KRONECKER PRODUCT}

The derivation of the master stability function (MSF) for zero-lag synchrony (see Chapter 3) and of MSF for group synchrony (see Chapter 5) relies on the Kronecker product. The following rules are used (assuming appropriate dimensions of all involved matrices, in particular, the matrix products $\mathbf{A C}$ and $\mathbf{B D}$ have to be defined):

Rule for block matrix multiplication

$$
(\mathbf{A} \otimes \mathbf{B})(\mathbf{C} \otimes \mathbf{D})=\mathbf{A C} \otimes \mathbf{B D} .
$$

Commutativity

$$
\left(\mathbf{A} \otimes \mathbb{1}_{n}\right)\left(\mathbb{1}_{m} \otimes \mathbf{B}\right)=\left(\mathbb{1}_{m} \otimes \mathbf{B}\right)\left(\mathbf{A} \otimes \mathbb{1}_{n}\right) .
$$

Rule (B.2) follows from rule (B.1): Let $\mathbf{A}$ be a matrix of the dimension $m \times m$ and $\mathbf{B}$ a matrix of the dimension $n \times n$. Then $:\left(\mathbf{A} \otimes \mathbb{1}_{n}\right)\left(\mathbb{1}_{m} \otimes \mathbf{B}\right)=\left(\mathbf{A} \mathbb{1}_{m}\right) \otimes\left(\mathbb{1}_{n} \mathbf{B}\right)=\left(\mathbb{1}_{m} \mathbf{A}\right) \otimes\left(\mathbf{B} \mathbb{1}_{n}\right)=$ $\left(\mathbb{1}_{m} \otimes \mathbf{B}\right)\left(\mathbf{A} \otimes \mathbb{1}_{n}\right)$ where the multiplication rule (B.1) was used. 


\section{ACRONYMS}

aEIF adaptive exponential integrate-and-fire

DDE delay differential equation

ETDFC extended time-delayed feedback control

ISI inter-spike interval

Icm least common multiple

MSE master stability equation

MSF master stability function

SG speed gradient

SNIC saddle-node bifurcation on invariant cycle

SNIPER saddle-node infinite period

TDFC time-delayed feedback control

ZRA zero resistance amperemeter 


\section{LIST OF FIGURES}

2.1. Rewiring procedure in the Watts-Strogatz model. (a) $p=0$ (regular network). (b) $p=0.08$ (small-world network). (c) $p=1$ (random network). Parameters: $N=20, k=2$. Figure modified from [Lehnert, 2010] . . . . . . . . . . 28

2.2. Small-world network. Shortest path length $\mathscr{L} / \mathscr{L}(p=0)$ (blue symbols) and clustering coefficient $\mathscr{C} / \mathscr{C}(p=0)$ (red symbols) versus rewiring probability $p$. Parameters: $N=100, k=4$. Number of realizations: 70 . (Reproduction of Fig. 2 in [Watts and Strogatz, 1998]) . . . . . . . . . . . . . . 31

3.1. Master stability function (MSF) for the saddle-node infinite period (SNIPER) bifurcation model as given by Eqs. (4.5)-(4.7). $\tau=6.5, \sigma=0.35, b=0.95$. Figure modified from [Keane et al., 2012]. . . . . . . . . . . . . . 44

4.1. SNIPER model. Phase portraits and typical trajectories of the uncoupled system (4.1): (a) below the SNIPER bifurcation, $b=0.95$; (b) at the bifurcation, $b=1$; and (c) above the bifurcation, $b=1.05$. Labels: F: unstable focus; S: Saddle; N: stable node. (d) Time series of the coupled system (4.4) with $\tau=8$, $\sigma=0.4$, and $b=0.95$. The green line shows $x(t)$, the blue one $y(t) . \ldots 49$

4.2. Master stability function (MSF) for the SNIPER model. Largest Lyapunov exponent $\Lambda$ for different delay times: (a) $\tau=6$, (b) $\tau=6.5$, (c) $\tau=7$, and (d) $\tau=10 . v$ is the eigenvalue of the coupling matrix, and $\Lambda$ is the largest Lyapunov exponent. Other parameters: $K=0.35, b=0.95$. Figure reproduced from $[$ Keane et al., 2012] . . . . . . . . . . . . . . . . . . . . 52

4.3. Master stability function (MSF) $\Lambda$ for a fixed coupling strength of (a) $K=$ 0.25 , (b) $K=0.3$ and (c) $K=0.35$ in the plane of the real part $\operatorname{Re} v(\operatorname{Im} v=$ 0 ) and the delay time $\tau$. The horizontal red lines show the position of the critical delay time $\tau_{c} . b=0.95$. Figure reproduced from [Keane et al., 2012]. . 52 
4.4. Dynamics of a FitzHugh-Nagumo system in the excitable regime after being sufficiently perturbed from the stable fixed point A. (a) Phase space of the FitzHugh-Nagumo system. Dark green line: trajectory, red lines: nullclines. (b) Time series of a spike. Dark blue line: activator $u$, pink line: inhibitor $v$. A, B, C, D mark corresponding points in panels (a) and (b). Red arrows: Schematic depiction of the phase portrait close to the cubic nullcline. Parameters: $a=1.05, \epsilon=0.01$. Figure reproduced from [Schöll et al., 2009]. . . 54

4.5. MSF for the FitzHugh-Nagumo system as given by Eq. (3.15) with Eqs. (3.2) and (4.11). $K=0.3$ and $\tau=1$. Figure modified from [Lehnert et al., 2011a]. . 55

4.6. Schematic view of networks consisting of excitatory (gray arrows) and inhibitory links (blue arrows). (a) Small-world type: Regular ring structure of excitatory coupling and additional randomly added long-range inhibitory links. (b) Random excitatory network with additional inhibitory links. Parameters: $N=20, k=2, p=0.1$. Number of excitatory links $E=40$, number of inhibitory links $I=4$. Modified from [Lehnert et al., 2011a] . . . . . . . 57

4.7. Fraction $f(p)$ of synchronized networks $f$ vs. the probability of additional inhibitory links $p$ for MSFs where the stable region is the unit cycle. $N=100$. $k$ varies from 6 to 30. Thin black curve: Example fit of $f(p)$ according to Eq. (4.12) ( $p_{c}=0.20387, k_{s}=186$ ) for $k=24.500$ realizations for each value of $k$. Figure modified from [Lehnert et al., 2011a]. . . . . . . . . . . . 58

4.8. Critical value $p_{c}$ for different network sizes, (a) in dependence on $k / N$, (b) in dependence on $k$ : small-world networks with $N=60$ (blue circles), $N=100$ (purple squares), and $N=500$ (turquoise triangles). Random networks with $N=60$ (red crosses), $N=100$ (orange circles), and $N=500$ (yellow squares). (c) $p_{c}$ vs. $N$ for $k=10$ for a small-world network (red filled circles) and a random network (red empty squares); number of inhibitory links $\left\langle I_{c}\right\rangle$ vs. $N$ for constant $k$ for a small-world (blue empty circles) and a random (blue filled squares) network. 500 realizations for each data point. Figure modified from [Lehnert et al., 2011a]. . . . . . . . . . . . . . . . . . . . . . . 58

4.9. Fraction of synchronized networks $f$ in dependence on the probability of additional inhibitory links $p$ for coupling ranges of (a) $k=20$, (b) $k=40$, and (c) $k=50$ with 500 realization for each $p$ value. Corresponding eigenvalue spectra for (d) $k=20$, (e) $k=40$, and (f) $k=50$ with 50 realizations for each $p$ value. The turquoise shaded regions represent the stable regions of the real part of the MSF. Red line in panels (a) and (d) marks the parameters used in Fig. 4.10. Parameters: $N=200, K=0.3, \tau=6.5, b=0.95$. Figure reproduced from [Keane et al., 2012]. . . . . . . . . . . . . . . . . . . . . 61 
4.10. Normalized histograms (depicted in blue) of eigenvalue spectra for 1000 realizations of (a) a small-world network and (b) a random network with the same number of nodes, $N=200$; the same number of excitatory links, $k=$ 20; and the same probability of inhibitory links, $p=0.23$. Turquoise shading: Stable region of the MSF for $K=0.3$ and $\tau=6.5$. Green lines in (a): Position of eigenvalues of a small-world network for $p=0$ according to Eq. (2.15). Green histogram in (b): histogram of eigenvalue spectra for 1000 realizations of a random network for $p=0$. Pink line in (b): eigenvalue distribution $\rho(v)$ according to Eq. (4.14). Number of bins is 1000. Figure reproduced and extended from $[$ Keane et al., 2012] . . . . . . . . . . . . . . . . . . . 62

5.1. (a) Schematic diagram of two groups visualizing parameters and dynamical variables as in Eq. (5.2) for multipartite topologies. (b) The corresponding synchronization manifold according to Eq. (5.6). Figure modified from [Dahms et al., 2012] . . . . . . . . . . . . . . . . . . . . . . 71

5.2. Master stability function (MSF) for group synchrony according to Eq. (5.15) for the SNIPER model (see Sec. 4.1.2, Eq. (4.4)) in the oscillatory regime for $M=1,2,3,4$ in panels (a) and (e), (b) and (f), (c) and (g), and (d) and (h), respectively. Black circles mark the longitudinal eigenvalues. Top: $\tau^{(k)} \equiv$ $\tau=3.4$, bottom: $\tau^{(k)} \equiv \tau=60$. Other parameters: $\sigma^{(k)} \equiv \sigma=0.1, b^{(k)} \equiv b=$ 1.05 , where $b^{(k)}$ is the bifurcation parameter of the SNIPER model for the $k$ th group. . . . . . . . . . . . . . . . . . . . 75

5.3. (a) Schematic diagram of two groups visualizing parameters and dynamical variables as in Eq. (5.30) for multiple commuting coupling matrices. Solid arrows denote coupling between the groups; dashed arrows correspond to coupling of nodes in the same group. (b) The corresponding synchronization manifold according to Eq. (5.31). Figure modified from [Dahms et al., 2012]. . . . . . . . . . . . . . . . . . . . . . . 79

5.4. (a)-(c): Master stability function (MSF) for networks of FitzHugh-Nagumo oscillators governed by Eq. (5.36) in the $\left(\operatorname{Re} v_{A}, \operatorname{Re} v_{B}\right)$ plane for $\operatorname{Im} v_{A}=\operatorname{Im} v_{B}=$ 0 and different delay times. The black dots denote the location of the eigenvalue pairs for the example topology (5.38). (d)-(i): Time series of the dynamics in the first (red) and second (blue) group. Parameters: (a),(d),(e): zero-lag synchronization $\left(\tau_{B}^{(k)}=3\right)$, (b),(f),(g): anti-phase synchronization $\left(\tau_{B}^{(k)}=2\right)$, (c),(h),(i): synchronized bursting $\left(\tau_{B}^{(k)}=3.2\right)$. Other parameters: $\sigma_{A}^{(k)}=\sigma_{B}^{(k)}=0.5, \tau_{A}^{(k)}=3, \epsilon=0.01, a=1.3$ (groups $k=0,1$ ). Figure from [Dahms et al., 2012] . . . . . . . . . . . . . . . . . . . . 82 
5.5. Supercritical Hopf bifurcation. Radius of oscillations vs. the bifurcation parameter $\lambda$. Solid and dashed lines mark stable and unstable states, respectively. . . . . . . . . . . . . . . . . . . . . . . . . 86

5.6. Schematic view of (a) zero-lag synchronization ( $m=0, M=1)$, (b) the splay state ( $m=1, M=4)$, (c) anti-synchronization ( $m=2, M=2)$, and (d) the reversed splay state ( $m=3, M=4)$. Each cluster consists of the same number of nodes. For convenience, the common radii of the different states are depicted as equal; however, according to Eq. (5.48) the common radius depends on $m$ and thus on the state . . . . . . . . . . . . . . . 88

5.7. Stability of synchrony for a homogenous population of coupled aEIF neurons as given by Eq. (5.70). The maximum Lyapunov exponent $\Lambda$ (color bar) is plotted as a function of the eigenvalues $v$ of the coupling matrix G. Panels (a), (b) and (c) show the results for strong adaptation $a_{w}$, and panels (d), (e) and (f) depict the results for weak subthreshold adaptation $a_{w}$. The networks are (a) and (d) excitation dominated, (b) and (e) balanced; and (c) and (f) inhibition dominated regimes. Parameters: $\lambda=5, \tau=0.3, \tau_{w}=10$, $\tau_{s}=0.3, V_{t h}=5, V_{r}=-5, b_{w}=2.5$. The external input $I$ is chosen such that the oscillation period is always equal to 5 . Circles indicate the unit disc in the eigenvalue plane (insets show blow-up). Figure from [Ladenbauer et al., 2013]. . . . . . . . . . . . . . . . . . . . . . 96

5.8. Stability of group synchrony in a network of excitatory $(\mathscr{E})$ and inhibitory $(\mathscr{I})$ neurons which differ in their local dynamics. (a) Scheme of the network according to Eq. (5.71). Solid green and dashed orange arrows correspond to excitatory (positive sign) and inhibitory coupling (negative sign), respectively. (b) Evolution of the membrane voltage $V$ for three different dynamical scenarios A, B, and C in the left, middle, and right column, respectively. Orange and green lines mark the voltage $V$ of synchronized excitatory and inhibitory neurons, respectively. The parameter sets for the scenarios A, B, and C are chosen according to Table 5.1. (c) Master stability function (MSF) for each of the three scenarios in (b). The maximum Lyapunov exponent is shown as a function of the real parts of the eigenvalues $v_{A}^{(k)}$ and $v_{B}^{(k)}$. White rectangles indicate the unit square in the eigenvalue plane. (d) Master stability function (MSF) inside the unit square as in (c), but with $a_{w}^{(\mathscr{E})}=0.1,0.2,0.3,0.4,0.5$ (top, left to right) and $b_{w}^{(\mathscr{E})}=0.5,1,1.5,2,2.5$ (bottom, left to right). Figure from [Ladenbauer et al., 2013]. . . . . . . . . . 98 
6.1. (a) Approximately synchronized spiking pattern (red dots) and (b) order parameter $R$ (blue line) vs. time for a small-world network with a delay distribution with standard deviation $\sigma_{\tau}=0.2$. Parameters: $N=50, p=0.51$, $k=2, K=1.0, a=1.3, \varepsilon=0.01$ and $\tau_{\mu}=5$. The initial condition is $u_{i}=-a$, $v_{i}=a-a^{3} / 3$ for all $i=0, \ldots, 49$. The history function of all nodes is the spiking state. Figure from [Cakan et al., 2014]. . . . . . . . . . . . . . . . . 107

6.2. Spiking patterns showing traveling disruptions in a regular ring network with $N=50$ nodes and $\sigma_{\tau}=0.2$. Red dots indicate spikes. Other parameters as in Fig. 6.1. Figure from [Cakan et al., 2014]. . . . . . . . . . . . . . . 108

6.3. Spiking pattern showing partial amplitude death in a small-world network $(N=50, p=0.51)$ for a delay distribution with standard deviation of $\sigma_{\tau}=$ 0.12. Red dots mark spikes. Other parameters as in Fig. 6.1. Figure from [Cakan et al., 2014]. . . . . . . . . . . . . . . . . . . . . . . 108

6.4. $\quad$ (a) Time series of $u$ (red solid) and $v$ (blue dotted), and (b) phase space of a single node undergoing amplitude death. The node is part of a small-world network ( $N=50, p=0.51$ ) with $\sigma_{\tau}=0.15$. (b) Trajectory (red solid) and $u$ nullcline (blue dashed line). Other parameters as in Fig. 6.1. Figure from [Cakan et al., 2014]. . . . . . . . . . . . . . . . . . . . . . . . . . 109

6.5. Probability of spiking $p_{s}$ (solid lines) and of highly synchronized spiking $p_{h}$ (dotted lines) vs. standard deviation of delay distribution $\sigma_{\tau}$ for (a) regular ring networks with $k=2$, i.e, each node is connected to its nearest and nextnearest neigbor to the left and to the right, (b) small-world networks with $k=2$ and $p=0.51$, and (c) random networks with $p=0.51$ and different network sizes $N=20$ (blue), $N=50$ (red), $N=100$ (green). Other parameters as in Fig. 6.1. Figure from [Cakan et al., 2014] . . . . . . . . . . . . . . 111

6.6. Spiking patterns showing subnetwork synchronization in a regular ring network with $N=50$ nodes for different $\sigma_{\tau}$ : (a)-(d) $\sigma_{\tau}=0.1$; (e),(f) $\sigma_{\tau}=0.13$. Panel (a),(c),(e): Red dots indicate spikes. Panel (b),(d),(f): Kuramoto order parameter $R$. Note that (c),(d) show the same simulation as (a),(b) for a longer time series. Other parameters as in Fig. 6.1. Figure from [Cakan et al., 2014]. . . . . . . . . . . . . . . . . . . . . . . . . . . 112

6.7. Example of a small-world network, where inner connections (red arrows) have a delay $\tau_{1}$, while the outer connections of the underlying regular ring (green arrows) are characterized by a delay $\tau_{2}$. Figure from [Cakan et al., 2014]. . . . . . . . . . . . . . . . . . . . . . . . . . . . . . . . 114

6.8. Interspike intervals (ISIs) in a small-world network plotted vs. the delay $\tau_{1}$ of the inner connections. (a) Simulations. (b) Mathematical reconstruction according to Eq. (6.1.3). $\tau_{2}=6, N=20, k=2, p=0.51$. Other parameters as in Fig. 6.1. Figure from [Cakan et al., 2014]. . . . . . . . . . . . . . . . . 114 
6.9. Interspike intervals (ISIs) (red dots) and delays (blue dots) of a small-world network. $N=50, \sigma_{\tau}^{(1)}=\sigma_{\tau}^{(2)}=\sigma_{\tau}=0.01, p=0.51$. Other parameters as in Fig. 6.1. Figure from [Cakan et al., 2014] . . . . . . . . . . . . . . . . . . 117

6.10. Interspike intervals (ISIs) (red dots) and delay distributions (blue dots) vs. $\sigma_{\tau} \equiv \sigma_{\tau}^{(1)}=\sigma_{\tau}^{(2)}$ for different combinations of mean delay times. (a), (b): small-world network ( $k=2, p=0.51)$, (c): regular ring network $(k=2)$. Mean delay times are $\tau_{1}=6$ and $\tau_{2}=8$ for (a) and (c), and $\tau_{1}=5$ and $\tau_{2}=10$ for (b). $N=50$. Other parameters as in Fig. 6.1. Figure from [Cakan et al., 2014]. . . . . . . . . . . . . . . . . . . . . . . . . . . . 118

6.11. Dynamics for a bimodal delay distribution in a regular ring with $k=2$ for different $\sigma_{\tau}$ : (a)-(c) $\sigma_{\tau}=0.05$; (d)-(f) $\sigma_{\tau}=0.08$. (a) and (d): time series of $u_{0}$ (dark red) and $v_{0}$ (light blue); (b) and (e): phase portraits of a single spiking node $\left(u_{0}, v_{0}\right)$ : trajectory (red solid) and $u$-nullcline (blue dashed); (c) and (e): spiking patterns. Mean delay times $\tau_{1}=6, \tau_{2}=8$. Other parameters as in Fig. 6.1. Figure from [Cakan et al., 2014]. . . . . . . . . . . . . . . . 119

6.12. Experimental set up and time series of electrochemical oscillations. (a) Experimental apparatus with multi-channel addressable feedback, $R_{p}$ is the channel resistance of $650 \Omega$. ZRA: zero resistance amperemeter. (b) Electrochemical dissolution time series showing smooth oscillators at a potential of $V_{0}=1.105 \mathrm{~V}$. (c) Time series of relaxation oscillators at $V_{0}=1.2 \mathrm{~V}$. Figure from [Blaha et al., 2013]. . . . . . . . . . . . . . . . . . . . . 122

6.13. Collective frequency $\Omega_{m}$ versus the time delay $\tau$ for the smooth oscillations. Red squares, blue triangles, and green circles represent a reverse splay state, an in-phase state, and a splay state, respectively. (a) Experimental data, $V_{0}=$ $1.105 \mathrm{~V}, K=0.15$. The arrows indicate an increase or decrease of $\tau$ during the measurement. (b) Solution continuation of Eq. (6.10) using DDE-BIFTOOL (symbols) and stable solutions of Eq. (6.12) (lines) where the stability is calculated according to Eq. (6.13). Parameters: $\lambda=1.1025, \omega=3.4228, K=0.3$, $N=4$. Figure from [Blaha et al., 2013]. . . . . . . . . . . . . . . . 125

6.14. Experimental time series (top) and schematic diagram (bottom) of an (a) in-phase state, (b) a 2-cluster state, (c) a reverse splay state, and (d) a splay state as shown in Fig. 6.13(a). The schematics show phase relations between oscillators on the phase ring. Oscillator colors in the schematic correspond to the colors in the times series: $\varphi_{0}$ is shown in blue, $\varphi_{1}$ is black, $\varphi_{2}$ is red, and $\varphi_{3}$ is green. Parameters: $V_{0}=1.105 \mathrm{~V}, K=0.15$; time delays: (a) $\tau=$ $1.05 \times 2 \pi / \omega$, (b) $0.5 \times 2 \pi / \omega$, (c) $1.2 \times 2 \pi / \omega$, and (d) $0.8 \times 2 \pi / \omega$ with $\omega=3.4228$ $\mathrm{rad} / \mathrm{s}$. Figure from [Blaha et al., 2013]. . . . . . . . . . . . . . . . 128 
6.15. Phase interaction function $H_{\varphi}$ in panels (a) and (b) for $V_{0}=1.105 \mathrm{~V}$ and $V_{0}=$ $1.2 \mathrm{~V}$, respectively; radial interaction function $H_{r}$ in panels (c) and (d) for $V_{0}=1.105 \mathrm{~V}$ and $V_{0}=1.2 \mathrm{~V}$, respectively. Experimentally obtained data is shown as red dots. A 5-term Fourier fit from evenly sampled data is shown by the black curve. Figure from [Blaha et al., 2013] . . . . . . . . . . . . . 130

6.16. Dynamics in dependence on the time delay $\tau$ for the relaxation oscillations. (a) Experimental data, $V_{0}=1.2 \mathrm{~V}, K=0.10$. The starting state is marked by a black arrow. The increasing and decreasing of $\tau$ during the experiment is shown with arrows. (b) Solutions of Eq. (6.17) using the continuation tool DDE-BIFTOOL (markers) and stable solutions of Eq (6.19) (lines) where the stability is given by Eq. (6.25). In-phase, 2-cluster, compressed splay, reverse splay, compressed reverse splay and open 2-cluster states are represented by upward-triangles, diamonds, open circles, squares, open squares and downward-triangles, respectively. The interaction functions $H_{r}$ and $H_{\varphi}$ are chosen as in Eq. (6.17) and Tab. 6.1. Parameters: $\lambda=2.890, K=0.189$ and $\omega=2.430$. Figure from $[$ Blaha et al., 2013] . . . . . . . . . . . . . 132

6.17. Experimental time series (top) and schematic diagram (bottom) of secondary states: (a) a compressed splay state, (b) a reverse compressed splay state, (c) an open 2-cluster state, (d) a compressed 2-cluster state. Parameters: $V_{0}=$ $1.2 \mathrm{~V}, K=0.10$; time delays: (a) $\tau=1.11 \times 2 \pi / \omega$, (b) $0.82 \times 2 \pi / \omega$, (c) $0.59 \times 2 \pi / \omega$ with $\omega=2.43 \mathrm{rad} / \mathrm{s}$ as in Fig. 6.16 and (d) $0.68 \times 2 \pi / \omega$ with $\omega=2.492 \mathrm{rad} / \mathrm{s}$. Figure from [Blaha et al., 2013]. . . . . . . . . . . . . . . . 133

8.1. Real part $\operatorname{Re} \Lambda$ of the eigenvalue of the unstable focus with standard TDFC vs. $K$ and $\tau . \lambda=0.5, \omega=\pi$. Figure modified from [Hövel, 2009]. . . . . . . 148

8.2. Adaptive control of the fixed point: (a) Time series $x(t)$ and (b) feedback gain $K(t)$ for different initial conditions: $x(0) \in[0.02,0.5]$ in steps of 0.02 (from light green to dark blue; in panel (b) from top to bottom), $y(0)=0$. Blue shaded region: $K$-interval of successful control for TDFC. Parameters: $\lambda=$ $0.5, \omega=\pi, \gamma=1, \tau=1$. Figure from [Lehnert et al., 2011b]. . . . . . . . . 149

8.3. Transient time $t_{c}$ after which the control goal is reached in dependence on the delay time $\tau$ for TDFC (black circles) and ETDFC with $R=0.35$ (blue crosses), and $R=0.95$ (red squares). The dark purple, bright purple, and red shaded regions denote the possible range of $\tau$ for $R=0,0.35$, and 0.95, respectively. Parameters as in Fig. 8.2. Figure from [Lehnert et al., 2011b]. . . 151 
8.4. Adaptive control of the fixed point for slowly drifting system parameter $\lambda$. (a) Adaptive adjustment of $K$ in the $(\lambda, K)$-plane. Blue shaded region: region of stability of the standard TDFC control. Red line with arrow: adaptation of feedback gain $K$ if $\lambda$ is slowly changed $(\lambda(t)=0.01+1.8 \sin (0.001 t)$ ) (b) Corresponding time series $K(t)$ (blue solid line) and $\lambda(t)$ (dashed red line). Other parameters as in Fig. 8.2. Figure from [Lehnert et al., 2011b] . . . . . . . 152

8.5. Robustness to noise. (a): Red curve: ensemble average $\langle x(t)\rangle$ of 200 realizations; blue curve: $x(t)$ for one example trial; green curve: corresponding standard deviation $\sigma_{x(t)}$ of $x(t)$ for a fixed noise intensity $D=0.1$. (b): Green crosses: standard deviation $\sigma_{x(100)}$ of $\langle x(t=100)\rangle$; dashed blue line: standard deviation of the input noise given by $D$; red curve: asymptotic value $K^{*}$ of the feedback gain. Parameters: $\gamma=0.001, x(0)=0.05, y(0)=0$. Other parameters as in Fig.8.2. Figure from [Lehnert et al., 2011b]. . . . . . . . . . 153

8.6. Adaptive control of an unstable periodic orbit in the Rössler attractor Eq. (8.16). (a) Phase portrait (after a transient time of 150 time units). (b) Time series of $K(t)$ with adaptive control given by Eq. (8.18) as blue curve. The curve shows the goal function $Q$. Parameters: $a=0.2, b=0.2, \mu=6.5, \gamma=0.1$, $\tau=5.91679$. Figure from [Lehnert et al., 2011b]. . . . . . . . . . . . 157

8.7. Adaptive control of the Rössler system. Red circles: fraction of realizations $f_{c}$ where the adaptive control algorithm stabilized the orbit versus the adaptation gain $\gamma$; blue crosses: Average time $t_{c}$ after which the control goal is reached versus $\gamma$; dotted blue lines: error bars (standard deviation) corresponding to $t_{c}$. Other parameters as in Fig. 8.6. Total number of realizations for each value of $\gamma:$ 100. Figure from [Lehnert et al., 2011b]. . . . . . . . 158

9.1. Goal functions for cluster synchronization. (a) Goal function $Q_{1}$ for zero-lag synchronization. (b) Goal function $1-f_{2}(\varphi)$ for antisynchronization without penalty terms. (c) Goal function $Q_{2}$ for antisynchronization. Blue line: 0contour line, i.e., minium of the depicted function. $N=2$. . . . . . . 163

9.2. Adaptive control of 3-cluster $(m=2,4)$ state in a network of 6 nodes according to Eq. (9.5). (a) Absolute values $r_{j}=z_{j}$. (b) Phase differences $\Delta_{j}=\varphi_{j+1}-\varphi_{j}$; blue dashed line: phase difference $\Delta_{j}=4 \pi / 3$ corresponding to a 3-cluster state with $m=2$. (c) Temporal evolution of $\beta$; blue dashed line: $\beta_{2}$ with $\Omega_{2}=1.03$. (d) Goal function. $K=0.08, \tau=0.52 \pi, N=6, \gamma=1$, $\omega_{i=0, \ldots, N-1}=1, \lambda_{i=0, \ldots, N-1}=0.1$. Figure reproduced from [Selivanov et al., 2012]. . . . . . . . . . . . . . . . . . . . . . . 165 
9.3. Same as in Fig. 9.2 but for nonidentical oscillators. Parameters $\lambda_{j}$ and $\omega_{j}$ are chosen from a Gaussian distribution with $1 \%$ standard deviation and mean values $\lambda=0.1$ and $\omega=1$, respectively. Here: $\omega_{0}=1.0192, \omega_{1}=1.0093$, $\omega_{2}=0.9780, \omega_{3}=0.9982, \omega_{4}=0.9799, \omega_{5}=0.9930, \lambda_{0}=0.0988 \lambda_{1}=0.1017$, $\lambda_{2}=0.1004, \lambda_{3}=0.09914, \lambda_{4}=0.1008, \lambda_{5}=0.1000$. Figure reproduced from [Selivanov et al., 2012]. . . . . . . . . . . . . . . . . . . . . . . . . . 166

9.4. Same as in Fig. 9.3 but for $5 \%$ standard deviation, i.e., $\omega_{0}=0.9395, \omega_{1}=$ 0.9288, $\omega_{2}=1.0495, \omega_{3}=0.9796, \omega_{4}=1.0160, \omega_{5}=0.9909, \lambda_{0}=0.0975, \lambda_{1}=$ 0.0956, $\lambda_{2}=0.1090, \lambda_{3}=0.1018, \lambda_{4}=0.0936, \lambda_{5}=0.1147$. Figure reproduced from [Selivanov et al., 2012] . . . . . . . . . . . . . . . . . . . . . 167

9.5. Stability as a function of coupling strength $K$ and delay $\tau$ for (a) $m=0$ ( $M=$ 1, zero-lag synchronization), (b) $m=1$ ( $M=4$, splay state), (c) $m=2$ ( $M=$ 2 , antisynchronization), and (d) $m=3$ ( $M=4$, splay-states). In the white areas no solution of Eq. (9.27) with $r_{0, m}^{2}>0$ exists. In all panels, $\beta^{*}=\beta_{m=2}$ according to Eq. (9.33), i.e., the $\beta$ value which stabilizes the $m=2$ state in the whole $(K, \tau)$-plane. $\omega_{i}=1, \lambda_{i}=0.1, i=0, \ldots, N-1$. Other parameters as in Fig. 9.3. . . . . . . . . . . . . . . . . . . . . . . 174

9.6. Fraction $f_{c}$ of successfully controlled networks and control time $t_{c}$ as a function of coupling strength $K$ and delay $\tau$ for (a),(d) zero-lag synchronization $(M=1, m=0)$; (b),(e) antisynchronization ( $M=2, m=2)$; and (c),(f) splaystates ( $M=4, m=1$ and $m=3$ ), respectively. White areas in (d), (e), and (f) correspond to parameter values where no realization was successful within $t<1000$. Dark blue lines correspond to contour lines of $1 /|\operatorname{Re} \Lambda|$ according to Eq. (9.35). $N=4.20$ realizations. Other parameters as in Fig. 9.2 . . . . . . 177

9.7. Adaptive control of 3-cluster state in a network of 6 nodes by simultaneously tuning $K, \beta$ and $\tau$ according to Eqs. (9.5), (9.38), and (9.37), respectively. (a) common radii $r_{j}$; (b) phase differences $\Delta_{j}=\varphi_{j}-\varphi_{j+1}$; (c) temporal evolution of control parameters $\beta$ (red line), $K$ (blue line), $\tau$ (green line), (d) goal function. $\gamma_{\beta}=\gamma_{K}=10, \gamma_{\tau}=0.05$. Other parameters as in Fig. 9.2. . . . . 179

10.1. Schematic view of the interplay of topology and local dynamics in a adaptive network. Figure modified from Fig. 1 in [Gross and Blasius, 2008] . . . . . . . 185

10.2. Evolution of the network topology with the goal to achieve an 8-cluster state. Black: excitatory weighted links; blue: inhibitory weighted links. Node colors denote phase differences with respect to the first node. Parameters: $\lambda=$ $0.1, \omega=1, c=0.01, K=0.1, \tau=\pi, \gamma_{G}=10, N=40, M=8$. Figure from [Lehnert et al., 2014]. 
10.3. Control of an 8-cluster state: (a) radii $r_{j}$, (b) phase difference $\varphi_{j}-\varphi_{0}$ with respect to the first node, (c) coupling weights $G_{i j}$, and (d) goal function $Q_{8}$ and its reduced part $q_{8}$ (excluding the unity-row-sum term). Control starts at $t=0$. The vertical dotted line at $t=80$ is explained in Sec. 10.6.2. Parameters as in Fig. 10.2. Figure from [Lehnert et al., 2014] . . . . . . . . . . . 189

10.4. Fraction $f_{c}$ of successfully controlled networks and control time $t_{c}$ as a function of coupling strength $K$ and delay $\tau$ for (a),(d) zero-lag synchronization $(M=1)$, (b),(e) a 3-cluster state $(M=3)$, and (d),(f) a splay-states $(M=15)$, respectively. White areas in (d) correspond to parameter values where the control is unsuccessful for all realizations. Averaged over 20 realizations. $N=15, \gamma_{G}=1$. Other parameters as in Fig. 10.2 . . . . . . . . . . . 190

10.5. Average link strength $\bar{G} \frac{2 \pi}{M} n$ versus phase differences $\Delta_{i j}=\lim _{t \rightarrow \infty}\left[\varphi_{i}(t)-\varphi_{j}(t)\right]$ in the final state as defined in Eq. (10.9) for different values of $\tau$ : (a) $\tau=0$ and $\tau=2 \pi$, (b) $\tau=\pi / 2$ and $\tau=3 \pi / 2$, and (c) $\tau=\pi$ and $\tau=3 \pi . N=30, M=10$, $K=0.08$. Average over 100 realizations. Other parameters as in Fig. 10.2. Figure from [Lehnert et al., 2014]. . . . . . . . . . . . . . . . . . . . 193

10.6. Average link strength $\bar{G} \frac{2 \pi}{M} n$ versus phase differences $\Delta_{i j}=\lim _{t \rightarrow \infty}\left[\varphi_{i}(t)-\varphi_{j}(t)\right]$ in the final state as defined in Eq. (10.9) for different values of $\gamma_{G}$ : green empty squares: $\gamma_{G}=100$; red filled squares: $\gamma_{G}=100$; blue empty circles: $\gamma_{G}=100$; yellow filled circles: $\gamma_{G}=100 . N=30, M=10, K=0.08$. Average over 100 realizations. Other parameters as in Fig. 10.2 . . . . . . . . . . . 194

10.7. Stability as a function of common frequency $\Omega_{M}$ and radius $r_{0, M}^{2}$ for (a) vanishing higher Fourier coefficients, i.e., $a_{l}^{j}=b_{l}^{j}=0$ for $l>1$, (b) random higher Fourier coefficients, (c) constant higher Fourier coefficients, i.e, $a_{l}^{j}=$ $b_{l}^{j}=10$ for $l>1 . N=8, M=4$. Other parameters as in Fig. 10.2. . . . . . 199

10.8. Control of an 8-cluster state: Fourier coefficients as defined in Eq. (10.12): (a) $a_{1}^{j}$, (b) $a_{2}^{j}$, (c) $a_{3}^{j}$. At $t=0$, the control is switched on (first vertical dotted line). At $t=80, a_{2}^{j}$ and $a_{3}^{j}$ are set to random values (second vertical dotted line). Parameters as in Fig. 10.2 . . . . . . . . . . . . . . . . 201

10.9. Control of an 8-cluster state: Fourier coefficients as defined in Eq. (10.12): (a) $b_{1}^{j}$, (b) $b_{2}^{j}$, (c) $b_{3}^{j}$, (d) $b_{4}^{j}$. At $t=0$, the control is switched on (first vertical dotted line). At $t=80, b_{2}^{j}, b_{3}^{j}, b_{4}^{j}$ are set to random values (second vertical dotted line). Parameters as in Fig. 10.2. Figure from [Lehnert et al., 2014]. . 202 
10.10. Oscillation quenching of a splay state: (a) radii $r_{j}$, (b) phases $\varphi_{j}$, (c) coupling weights $G_{i j}$, and (d) goal function $Q_{12}$, its reduced part $q_{12}$ and the collective frequency $\Omega_{M} . N=12, M=12$. At $t=40$ the adaptive control is switched off and $a$ is set to $a=\frac{2 \omega}{N K}$. Other parameters as in Fig. 10.2. Figure from [Lehnert et al., 2014] . . . . . . . . . . . . . . . . . . . . . . . 203

10.11. Topology (a) before $(t=0)$ and (b) after control, i.e., after the vanishing of all transient effects. Black links: fixed links; green: adapted links. Color code of nodes: Phase difference $\Delta \varphi=\varphi_{j}-\varphi_{0}$ with respect to the first node. Parameters: $\tau=3, A / L=0.3, P / L=0.4, N=15, M=3$. Other parameters as in Fig. 10.2. Initial conditions: directed random network. Figure from [Lehnert et al., 2014] . . . . . . . . . . . . . . . . . . . . . . . . . 205

10.12. Restricted control of a 3-cluster state: (a) radii $r_{j}$, (b) phase difference $\varphi_{j}-\varphi_{0}$ with respect to the first node, (c) coupling weights $G_{i j}$, and (d) goal function $Q_{3}$ and it its reduced part $q_{3}$. Parameters as in Fig. 10.11. Figure from [Lehnert et al., 2014] . . . . . . . . . . . . . . . . . . . . . . . 206

10.13. Control of a subset of links. (a) Fraction $f_{c}$ of successfully controlled networks, and (b) time $t_{c}$ needed to reach the control goal as a function of the number of random links $P$ and the number of controlled links $A$, normalized by $L=N(N-1) .10$ realizations. $K=0.2$. Other parameters as in Fig. 10.11. Figure modified from [Lehnert et al., 2014]. . . . . . . . . . . . . . 206

10.14. Control of a subset of links for fixed ratio of (a) $P / L=0.4$, (b) $P / L=0.6$, and (c) $P / L=1$. Red circles: success rate $f_{c}$; blue circles: probability $p_{>1}$ that no node in the network has less than 2 incoming links which are adapted; blue line: $p_{>1}$ according to Eq. (10.22). $K=0.2 .80$ realizations for each value of A/L. Other parameters as in Fig. 10.11. . . . . . . . . . . . . . 207 


\section{LIST OF TABLES}

3.1. Dynamics within the synchronization manifold as determined by the longitudinal Lyapunov exponent $\Lambda\left(v_{0}\right)$ when numerically calculating the MSF. . . . . 43

5.1. Parameters used for Figs. 5.8(b) and (c). . . . . . . . . . . . . . . . . . . . . 102

6.1. Fourier coefficients of the coupling function used in Eq. (6.17). . . . . . . . . . 131 


\section{BIBLIOGRAPHY}

Abrams, D. M. and Strogatz, S. H. (2004), Chimera States for Coupled Oscillators, Phys. Rev. Lett. 93, 174102. (Cited on pages 27 and 215.)

Adamic, L. A. (1999), The Small World Web, Lecture Notes in Computer Science, volume 1696/1999, Springer Berlin / Heidelberg, ISBN 978-3-540-66558-8. (Cited on pages 12 and 21.)

Ahlborn, A. AND Parlitz, U. (2004), Stabilizing Unstable Steady States Using Multiple Delay Feedback Control, Phys. Rev. Lett. 93, 264101. (Cited on pages 17 and 146.)

AIHARA, K. AND SUZUKI, H. (2010), Theory of hybrid dynamical systems and its applications to biological and medical systems, Phil. Trans. R. Soc. A 368, 4893. (Cited on page 89.)

Albert, R. AND BARABASI, A. L. (2002), Statistical mechanics of complex networks, Rev. Mod. Phys. 74, 47. (Cited on pages 11, 12, 23, 31 and 184.)

ALBERT, R., JEONG, H., AND BARABASI, A. L. (2000), Error and attack tolerance of complex networks, Nature 406, 378. (Cited on pages 13 and 184.)

AmAnn, A., SCHÖLL, E., AND JuST, W. (2007), Some basic remarks on eigenmode expansions of time-delay dynamics, Physica A 373, 191. (Cited on page 178.)

ANDERson, R. H. AND MAY, R. M. (1992), Infectious diseases of humans: dynamics and control., Oxford University Press, Oxford and New York. (Cited on page 32.)

Arenas, A., DíAz-Guilera, A., Kurths, J., Moreno, Y., And Zhou, C. (2008), Synchronization in complex networks, Phys. Rep. 469, 93, ISSN 0370-1573. (Cited on page 13.)

Arenas, A., DíAz-Guilera, A., And Pérez-Vicente, C. J. (2006a), Synchronization Reveals Topological Scales in Complex Networks, Phys. Rev. Lett. 96, 114102. (Cited on pages 13 and 14.) 
Arenas, A., DíAz-Guilera, A., And Pérez-Vicente, C. J. (2006b), Synchronization Reveals Topological Scales in Complex Networks, Phys. Rev. Lett. 96, 114102. (Cited on page 32.)

Ashwin, P., Orosz, G., Wordsworth, J., And Townley, S. (2007), Dynamics on Networks of Cluster States for Globally Coupled Phase Oscillators, SIAM J. Appl. Dyn. Syst. 6, 728. (Cited on page 67.)

AsL, F. M. AND Ulsoy, A. G. (2003), Analysis of a system of linear delay differential equations, ASME J. Dyn. Syst., Meas., Control 125, 215. (Cited on pages 178 and 217.)

Astolfi, A., Karagiannis, D., AND Ortega, R. (2008), Nonlinear and Adaptive Control with Applications, Springer, Heidelberg. (Cited on page 142.)

AtaY, F. M. (2003a), Distributed delays facilitate amplitude death of coupled oscillators, Phys. Rev. Lett. 91, 094101. (Cited on page 138.)

ATAY, F. M. (2003b), Total and partial amplitude death in networks of diffusively coupled oscillators, Physica D 183, 1, ISSN 0167-2789. (Cited on page 137.)

AtaY, F. M. (Editor) (2010), Complex Time-Delay Systems, Understanding Complex Systems, Springer, Berlin Heidelberg. (Cited on page 33.)

Aviad, Y., Reidler, I., Zigzag, M., Rosenbluh, M., And Kanter, I. (2012), Synchronization in small networks of time-delay coupled chaotic diode lasers, Opt. Express 20, 4352. (Cited on pages 14 and 67.)

Balanov, A. G., Janson, N. B., Postnov, D. E., And Sosnovtseva, O. V. (2009), Synchronization: From Simple to Complex, Springer, Berlin. (Cited on page 13.)

Balanov, A. G., Janson, N. B., AND SchÖlL, E. (2005), Delayed feedback control of chaos: Bifurcation analysis, Phys. Rev. E 71, 016222. (Cited on page 156.)

Banarjee, S. And Verghese, G. C. (2001), Nonlinear phenomena in power electronics: Bifurcations, chaos, control, and applications, Wiley-IEEE Press [Imprint]. (Cited on page 89.)

BARABASI, A. L. AND Albert, R. (1999), Emergence of Scaling in Random Networks, Science 286, 509. (Cited on page 12.)

Barabasi, A. L. and Bonabeau, E. (2003), Scale-free networks, Sci. Am. 288, 50. (Cited on page 21.) 
Barahona, M. And Pecora, L. M. (2002), Synchronization in Small-World Systems, Phys. Rev. Lett. 89, 054101. (Cited on pages 12 and 21.)

BARbaY, S., Kuszelewicz, R., AND YaCOMOtTI, A. M. (2011), Excitability in a semiconductor laser with saturable absorber, Opt. Lett. 36, 4476. (Cited on page 47.)

Bassett, D. S., Meyer-Lindenberg, A., Achard, S., Duke, T., And Bullmore, E. T. (2006), Adaptive reconfiguration of fractal small-world human brain functional networks, Proc. Natl. Acad. Sci. USA 103, 19518. (Cited on page 12.)

Battogtokh, D., Aihara, K., And Tyson, J. J. (2006), Synchronization of Eukaryotic Cells by Periodic Forcing, Phys. Rev. Lett. 96, 148102. (Cited on page 89.)

Bazhenov, M. And Timofeev, I. (2006), Thalamocortical oscillations, Scholarpedia $\mathbf{1}$, 1319. (Cited on page 81.)

Beck, O., Amann, A., Schöll, E., Socolar, J. E. S., AND Just, W. (2002), Comparison of time-delayed feedback schemes for spatio-temporal control of chaos in a reactiondiffusion system with global coupling, Phys. Rev. E 66, 016213. (Cited on page 147.)

Belik, V., Geisel, T., AND Brockmann, D. (2011), Natural Human Mobility Patterns and Spatial Spread of Infectious Diseases, Phys. Rev. X 1, 011001. (Cited on page 32.)

Belykh, I., Hasler, M., Lauret, M., ANd Nijmeijer, H. (2005), Synchronization and graph topology, Int. J. Bifurcation Chaos 15, 3423. (Cited on page 14.)

Belykh, I. AND Shilnikov, A. (2008), When Weak Inhibition Synchronizes Strongly Desynchronizing Networks of Bursting Neurons, Phys. Rev. Lett. 101, 078102. (Cited on page 15.)

BelzIG, W. (2005), Fluktuierender Strom - Quantenrauschen in mesoskopischen Systemen, Physik Journal 4, 75. Schoee Einfuerung in die full-counting-statistics auf deutsch!Aug./Sept. (Cited on page 14.)

Benoit, E. E., Callot, J. L., Diener, F., And Diener, M. M. (1981), Chasse au canard (première partie), Collect. Math. 32, 37. (Cited on page 65.)

Bernardo, M., Budd, C., Champneys, A. R., And Kowalczyk, P. (2008), Piecewisesmooth dynamical systems: theory and applications, volume 163, Springer. (Cited on page 89.)

Bick, C., Timme, M., And Kolodziejski, C. (2012), Adapting Predictive Feedback Chaos Control for Optimal Convergence Speed, SIAM J. Appl. Dyn. Syst. 11, 1310.(Cited on page 159.) 
BlaHA, K. (2013), Improving Reduced Variable Models for Complex Systems via Experiment, Ph.D. thesis, University of Virginia. (Cited on pages 121 and 123.)

Blaha, K., Lehnert, J., Keane, A., Dahms, T., Hövel, P., Schöll, E., And Hudson, J. L. (2013), Clustering in delay-coupled smooth and relaxational chemical oscillators, Phys. Rev. E 88, 062915. (Cited on pages 14, 16, 67, 103, 120, 122, 125, 128, 129, 130, 132, 133, 228 and 229.)

Blasius, B., Huppert, A., AND Stone, L. (1999), Complex dynamics and phase synchronization in spatially extended ecological systems, Nature (London) 399, 354. (Cited on pages 13, 14 and 67.$)$

Boccaletti, S., Bianconi, G., Criado, R., Del Genio, C. I., Gómez-Gardeñes, J., Romance, M., Sendiña NAdal, I., WANG, Z., And Zanin, M. (2014), The structure and dynamics of multilayer networks, Physics Reports 544, 1, ISSN 0370-1573. The structure and dynamics of multilayer networks. (Cited on page 12.)

Boccaletti, S., Hwang, D. U., Chavez, M., Amann, A., Kurths, J., and Pecora, L. M. (2006a), Synchronization in dynamical networks: Evolution along commutative graphs, Phys. Rev. E 74, 016102. (Cited on page 32.)

Boccaletti, S., Latora, V., Moreno, Y., Chavez, M., and Hwang, D. U. (2006b), Complex networks: Structure and dynamics, Phys. Rep. 424, 175, ISSN 0370-1573. (Cited on pages 11, 23 and 184.)

Brette, R. and Gerstner, W. (2005), Adaptive Exponential Integrate-and-Fire Model as an Effective Description of Neuronal Activity, J. neurophysiol. 94, 3637, ISSN 00223077. (Cited on page 94.)

BRUNEL, N. (2000), Dynamics of sparsely connected networks of excitatory and inhibitory spiking neurons, J. Comput. Neurosci. 8, 183. (Cited on page 90.)

Buldyrev, S. V., Parshani, R., Paul, G., Eugene Stanley, H., and Shlomo, H. (2010), Catastrophic cascade of failures in interdependent networks, Nature 464, 1025, ISSN 0028-0836. 10.1038/nature08932. (Cited on page 12.)

CAKAn, C., LehnerT, J., AND SChÖLl, E. (2014), Heterogeneous Delays in Neural Networks, Eur. Phys. J. B 87, 54. (Cited on pages 15, 103, 104, 107, 108, 109, 111, 112, 114, $117,118,119,227$ and 228.)

Cassandras, C. G., Pepyne, D. L., And WARdi, Y. (2001), Optimal control of a class of hybrid systems, IEEE trans. automat. contr. 46, 398. (Cited on page 89.) 
Chavez, M., Hwang, D. U., Amann, A., And Boccaletti, S. (2006), Synchronizing weighted complex networks, Chaos 16, 015106. (Cited on page 32.)

Chavez, M., Hwang, D. U., Amann, A., Hentschel, H. G. E., and Boccaletti, S. (2005), Synchronization is Enhanced in Weighted Complex Networks, Phys. Rev. Lett. 94, 218701. (Cited on pages 13, 184 and 193.)

CHEN, L. AND YU, X. (1999), On time-delayed feedback control of chaotic systems, IEEE Trans. Circuits Syst. Regul. Pap. 46, 767, ISSN 1057-7122. (Cited on page 159.)

Chen, Q., Chang, H., Govindan, R., Jamin, S., Shenker, S. J., and Willinger, W. (2002), The Origin of Power Laws in Internet Topologies Revisited, in Proceedings of the 21st Annual Joint Conference of the IEEE Computer and Communications Societies, IEEE Computer Society ISSN 0743-166X. (Cited on pages 12 and 31.)

Choe, C. U., Dahms, T., Hövel, P., AND SchöLl, E. (2010), Controlling synchrony by delay coupling in networks: from in-phase to splay and cluster states, Phys. Rev. E 81, 025205(R). (Cited on pages 13, 16, 18, 40, 68, 85, 88, 89, 120, 123, 130, 134, 138, 161, $167,169,172,175,178,181$ and 184.)

Choe, C. U., Dahms, T., Hövel, P., And Schöll, E. (2011), Control of synchrony by delay coupling in complex networks, in Proceedings of the Eighth AIMS International Conference on Dynamical Systems, Differential Equations and Applications, pages 292301, American Institute of Mathematical Sciences, Springfield, MO, USA. DCDS Supplement Sept. 2011. (Cited on pages 85 and 161.)

Choe, C. U., Jang, H., Ri, H. M., Dahms, T., Flunkert, V., Hövel, P., And Schöll, E. (2012), Simultaneous stabilization of periodic orbits and fixed points in delay-coupled Lorenz systems, Cybernetics and Physics 1, 155. (Cited on page 161.)

Choe, C. U., Kim, R.-S., Hövel, P., AND Schöll, E. (2014a), Optimal and resonant time-delayed feedback control of unstable steady states: Self-adaptive tuning of coupling phase, Int. J. Dynam. Control (available online) ISSN 2195-268X. (Cited on page 161.)

Choe, C. U., Kim, R.-S., Jang, H., Hövel, P., And Schöll, E. (2014b), DelayedFeedback Control with Arbitrary and Distributed Delay-Time and Noninvasive Control of Synchrony in Networks Coupled with Heterogeneous Delays, Int. J. Dynam. Control 2, 2. (Cited on page 162.)

CiOfFI, A. G., MARTIN, R. S., AND Kiss, I. Z. (2011), Electrochemical oscillations of nickel electrodissolution in an epoxy-based microchip flow cell, J. Electroanal. Chem. 659, 92, ISSN 1572-6657. (Cited on page 121.) 
CONNOR, J. A., WALTER, D., AND MCKown, R. (1977), Neural repetitive firing: modifications of the Hodgkin-Huxley axon suggested by experimental results from crustacean axons, Biophys. J. 18, 81, ISSN 0006-3495. (Cited on page 49.)

Corless, R. M., Gonnet, G. H., Hare, D. E. G., Jeffrey, D. J., And Knuth, D. E. (1996), On the Lambert W Function, Adv. Comput. Math 5, 329. (Cited on page 178.)

Curtu, R. (2010), Singular Hopf bifurcations and mixed-mode oscillations in a two-cell inhibitory neural network, Phys. D 239, 504. (Cited on page 210.)

Dahlem, M. A., Hiller, G., Panchuk, A., And Schöll, E. (2009), Dynamics of delaycoupled excitable neural systems, Int. J. Bifur. Chaos 19, 745. (Cited on pages 55 and 64.)

Dahms, T. (2011), Synchronization in Delay-Coupled Laser Networks, Ph.D. thesis, Technische Universität Berlin. (Cited on pages 13, 14, 15, 17, 32, 40, 68, 69, 72, 81, 85, 93, 161, 178 and 198.)

DAhMs, T., HöVel, P., AND SCHÖLL, E. (2007), Control of unstable steady states by extended time-delayed feedback, Phys. Rev. E 76, 056201. (Cited on pages 17, 146 and 155.)

DAHMS, T., LEHNERT, J., AND SCHÖLL, E. (2012), Cluster and group synchronization in delay-coupled networks, Phys. Rev. E 86, 016202. (Cited on pages 14, 15, 67, 68, 69, 70, 71, 72, 75, 79, 82, 93, 198 and 225.)

Dale, H. (1935), Pharmacology and Nerve-endings (Walter Ernest Dixon Memorial Lecture), Proc. R. Soc. Med. 28, 319. (Cited on page 56.)

Dayan, P. AND AвBOtT, L. F. (2005), Theoretical Neuroscience: Computational And Mathematical Modeling of Neural Systems, Computational Neuroscience, Massachusetts Institute of Technology Press, ISBN 9780262541855. (Cited on pages 32 and 48.)

De Solla Price, D. J. (1965), Networks of Scientific Papers, Science 149, 510. (Cited on pages 12 and 31.)

Deco, G., Ponce-Alvarez, A., Hagmann, P., Romani, G. L., Mantini, D., And CorBETTA, M. (2014), How Local Excitation-Inhibition Ratio Impacts the Whole Brain Dynamics, The Journal of Neuroscience 34, 7886. (Cited on page 15.)

Degesys, J., Rose, I., PATEl, A., AND NagPal, R. (2007), DESYNC: self-organizing desynchronization and TDMA on wireless sensor networks, in Proceedings of the 6th international conference on Information processing in sensor networks, pages 11-20, ACM. (Cited on page 13.) 
DesteXHe, A. (2009), Self-sustained asynchronous irregular states and Up-Down states in thalamic, cortical and thalamocortical networks of nonlinear integrate-and-fire neurons, J. Comput. Neurosci. 27, 493. (Cited on pages 90, 97, 99 and 100.)

Dhamala, M., JiRSA, V. K., AND Ding, M. (2004), Enhancement of Neural Synchrony by Time Delay, Phys. Rev. Lett. 92, 074104. (Cited on pages 13, 14, 15, 40, 81 and 184.)

D’Huys, O., JÜngling, T., AND KInZel, W. (2014), Stochastic switching in delay-coupled oscillators, arXiv. 1409.0479. (Cited on page 17.)

D’Huys, O., Zeeb, S., JÜngling, T., Heiligenthal, S., Yanchuk, S., And Kinzel, W. (2013), Synchronisation and scaling properties of chaotic networks with multiple delays, EPL 103, 10013. (Cited on pages 14, 17 and 40.)

di Bernardo, M., Garefalo, F., Glielmo, L., and Vasca, F. (1998), Switchings, bifurcations, and chaos in DC/DC converters, Circuits and Systems I: Fundamental Theory and Applications, IEEE Transactions on 45, 133. (Cited on page 89.)

Diaz-Guilera, A., Gómez-Gardenes, J., Moreno, Y., And Nekovee, M. (2009), Synchronization in random geometric graphs, International Journal of Bifurcation and Chaos 19, 687. (Cited on page 13.)

Earl, M. G. AND Strogatz, S. H. (2003), Synchronization in oscillator networks with delayed coupling: A stability criterion, Phys. Rev. E 67, 036204. (Cited on pages 83, 97 and 100.)

ECCLES, J. (1976), From electrical to chemical transmission in the central nervous system, Notes rec. R. Soc. Lond. 30, 219, ISSN 0035-9149. (Cited on page 56.)

Engel, A., Fries, P., And Singer, W. (2001), Dynamic predictions: Oscillations and synchrony in top-down processing, Nature Reviews Neuroscience 2, 704. (Cited on page 13.)

Engelborghs, K., LuZYAnina, T., AND Roose, D. (2002), Numerical bifurcation analysis of delay differential equations using DDE-Biftool, ACM Transactions on Mathematical Software 28, 1. (Cited on page 127.)

Engelborghs, K., LuZYAnina, T., AND SAMAeY, G. (2001), DDE-BIFTOOL v. 2.00: a Matlab package for bifurcation analysis of delay differential equations, Technical Report TW-330, Department of Computer Science, K.U.Leuven, Belgium. (Cited on page 127.)

Englert, A., Heiligenthal, S., Kinzel, W., And Kanter, I. (2011), Synchronization of chaotic networks with time-delayed couplings: An analytic study, Phys. Rev. E 83, 046222. (Cited on page 17.) 
Englert, A., Kinzel, W., Aviad, Y., Butkovski, M., Reidler, I., Zigzag, M., Kanter, I., AND Rosenbluh, M. (2010), Zero lag synchronization of chaotic systems with time delayed couplings, Phys. Rev. Lett. 104, 114102. (Cited on page 17.)

ERdős, P. And RÉNYI, A. (1959), On random graphs, Publ. Math. Debrecen 6, 290. (Cited on pages $11,21,29,106$ and 184.)

ERDős, P. AND RÉNYI, A. (1960), On the evolution of random graphs, Publ. Math. Inst. Hung. Acad. Sci 5, 17. (Cited on pages 12, 21, 29 and 106.)

Ermentrout, B., Pascal, M., And Gutkin, B. (2001), The effects of spike frequency adaptation and negative feedback on the synchronization of neural oscillators, Neural Comp. 13, 1285, ISSN 0899-7667. (Cited on page 95.)

Ermentrout, G. B. And Kopell, N. (1986), Parabolic Bursting in an Excitable System Coupled with a Slow Oscillation, SIAM j. appl. math. 46, 233. (Cited on page 49.)

Erneux, T. (2009), Applied delay differential equations, Springer. (Cited on page 33.)

ERnst, U., PAWElziK, K., AND GeISEL, T. (1998), Delay-induced multistable synchronization of biological oscillators, Phys. Rev. E 57, 2150. (Cited on page 32.)

Euler, L. (1741), Solutio problematis ad geometriam situs pertinentis, Commentarii academiae scientiarum Petropolitanae 8, 128. (Cited on page 11.)

FARMER, J. D. (1982), Chaotic attractors of an infinite-dimensional dynamical system, Physica D 4, 366. (Cited on page 33.)

FERrer I CANChO, R. AND SolÉ, R. V. (2001), The small world of human language, Proceedings of the Royal Society of London. Series B: Biological Sciences 268, 2261. (Cited on page 21.)

Fiedler, B., Flunkert, V., Georgi, M., Hövel, P., And Schöll, E. (2007), Refuting the odd number limitation of time-delayed feedback control, Phys. Rev. Lett. 98, 114101. (Cited on page 161.)

Fink, K. S., Johnson, G., Carroll, T. L., Mar, D., And Pecora, L. M. (2000), Three coupled oscillators as a universal probe of synchronization stability in coupled oscillator arrays, Phys. Rev. E 61, 5080. (Cited on page 37.)

FitzHugh, R. (1961), Impulses and physiological states in theoretical models of nerve membrane, Biophys. J. 1, 445. (Cited on pages 18, 47, 53 and 104.) 
Flunkert, V. (2011), Delay-Coupled Complex Systems, Springer Theses, Springer, Heidelberg, ISBN 978-3-642-20249-0. (Cited on page 32.)

FlunKert, V., FisCHER, I., AND SCHÖLL, E. (2013a), Dynamics, control and information in delay-coupled systems, Theme Issue of Phil. Trans. R. Soc. A 371, 20120465. (Cited on page 17.)

FLUNKERT, V. AND SCHÖLL, E. (2007), Suppressing noise-induced intensity pulsations in semiconductor lasers by means of time-delayed feedback, Phys. Rev. E 76, 066202. (Cited on page 161.)

FLUNKERT, V. AND SCHÖLL, E. (2011), Towards easier realization of time-delayed feedback control of odd-number orbits, Phys. Rev. E 84, 016214. (Cited on page 154.)

Flunkert, V., YANChuK, S., Dahms, T., AND SchÖll, E. (2010), Synchronizing distant nodes: a universal classification of networks, Phys. Rev. Lett. 105, 254101. (Cited on pages $14,17,32,51,55,64,65$ and 76.$)$

Flunkert, V., YanchuK, S., Dahms, T., AND Schöll, E. (2013b), Synchronizability of networks with strongly delayed links: a universal classification, Contemporary Mathematics. Fundamental Directions 48, 134. English version: J. of Math. Sciences (Springer), 2014. (Cited on page 17.)

Fortunato, S. (2010), Community detection in graphs, Phys. Rep. 486, 75. (Cited on page 24.)

FRADKOV, A. L. (1979), Speed-gradient scheme and its application in adaptive control problems, Autom. Remote Control 40, 1333. (Cited on pages 3, 4, 16, 142, 144 and 212.)

FradKov, A. L. (2005), Application of cybernetic methods in physics, Physics-Uspekhi 48, 103. (Cited on pages 16 and 142.)

Fradkov, A. L. (2007), Cybernetical Physics: From Control of Chaos to Quantum Control, Springer, Heidelberg, Germany. (Cited on pages 3, 4, 16, 142, 186 and 212.)

Fradkov, A. L., Miroshnik, I. V., And Nikiforov, V. O. (1999), Nonlinear and Adaptive Control of Complex Systems, Kluwer, Dordrecht. (Cited on page 142.)

Fradkov, A. L. AND Pogromsky, A. Y. (1998), Introduction to Control of Oscillations and Chaos, World Scientific, Singapore. (Cited on page 16.)

Fridman, E. (2014), Discrete-Time Delay Systems, in Introduction to Time-Delay Systems, Systems \& Control: Foundations \& Applications, pages 243-272, Springer International Publishing, ISBN 978-3-319-09392-5. (Cited on page 33.) 
FRIES, P. (2005), A mechanism for cognitive dynamics: neuronal communication through neuronal coherence, Trends in cognitive sciences 9,474 . (Cited on page 13.)

Fukuda, H., Nakamichi, N., Hisatsune, M., Murase, H., and Mizuno, T. (2007), Synchronization of Plant Circadian Oscillators with a Phase Delay Effect of the Vein Network, Phys. Rev. Lett. 99, 098102. (Cited on page 13.)

Ganopolski, A. And Rahmstorf, S. (2002), Abrupt Glacial Climate Changes due to Stochastic Resonance, Phys. Rev. Lett. 88, 038501. (Cited on page 47.)

GAO, J., LI, D., AND HAVLIN, S. (2014), From a single network to a network of networks, National Science Review 1, 346. (Cited on page 12.)

García-Ojalvo, J., Elowitz, M. B., And Strogatz, S. H. (2004), Modeling a synthetic multicellular clock: Repressilators coupled by quorum sensing, PNAS 101, 10955. (Cited on page 13.)

GefFert, P. M. (2015), Stochastic Non-Excitable Systems with Time Delay, BestMasters 2014, Springer. (Cited on page 32.)

GERSCHGORIN, S. A. (1931), Über die Abgrenzung der Eigenwerte einer Matrix, Izv. Akad. Nauk. SSSR 6, 749. (Cited on pages 83, 97 and 100.)

Gigante, G., Mattia, M., And Giudice, P. D. (2007), Diverse Population-Bursting Modes of Adapting Spiking Neurons, Phys. Rev. Lett. 98, 148101. (Cited on page 90.)

Golubitsky, M. AND SteWART, I. (2002), The symmetry perspective, Birkhäuser, Basel. (Cited on pages 14, 67 and 183.)

Goulding, D., Hegarty, S. P., Rasskazov, O., Melnik, S., Hartnett, M., Greene, G., MCINERNEY, J. G., RACHINSKII, D., AND HUYET, G. (2007), Excitability in a Quantum Dot Semiconductor Laser with Optical Injection, Phys. Rev. Lett. 98, 153903. (Cited on page 47.)

GraY, R. M. (2005), Toeplitz and Circulant Matrices: A Review., Found. Trends Commun. Inf. Theory 2. (Cited on page 27.)

Grebogi, C. (2010), Recent Progress in Controlling Chaos, Series on stability, vibration, and control of systems, World Scientific Publishing Company, Incorporated, ISBN 9789814291705. (Cited on pages 17 and 146.)

Gross, T. AND BlasiUs, B. (2008), Adaptive coevolutionary networks: a review, J. R. Soc. Interface 5, 259. (Cited on pages 16, 184, 185 and 231.) 
Grossman, J. W. AND ION, P. D. F. (1995), On a portion of the well-known collaboration graph, Congr. Numer. 108, 129. (Cited on page 31.)

Gu, K., Kharitonov, V. L., and Chen, J. (2003), Stability of Time-Delay Systems, Birkhauser Boston. (Cited on page 159.)

Gutiérrez, R., Amann, A., Assenza, S., Gómez-Gardeñes, J., Latora, V., And BocCALETTI, S. (2011), Emerging Meso- and Macroscales from Synchronization of Adaptive Networks, Phys. Rev. Lett. 107, 234103. (Cited on page 14.)

Guzenko, P. Y., Hövel, P., Flunkert, V., Fradkov, A. L., And Schöll, E. (2008), Adaptive Tuning of Feedback Gain in Time-Delayed Feedback Control, Proc. 6th EUROMECH Nonlinear Dynamics Conference (ENOC-2008), ed. A. Fradkov, B. Andrievsky, IPACS Open Access Library http://lib.physcon.ru (e-Library of the International Physics and Control Society). (Cited on pages 145 and 156.)

GuzenKo, P. Y., Lehnert, J., Fradkov, A. L., AND SChÖLl, E. (2013a), Adaptive control of networks of delay-coupled chaotic systems, in Proceedings of the 6th International Conference on Physics and Control (PhysCon 2013), IPACS Electronic library. (Cited on page 159.)

GuZENKo, P. Y., LeHnerT, J., AND SCHÖLL, E. (2013b), Application of Adaptive Methods to Chaos Control of Networks of Rössler Systems, Cybernetics and Physics 2, 15. (Cited on page 159.)

Hagerstrom, A. M., Murphy, T. E., Roy, R., Hövel, P., Omelchenko, I., And SchölL, E. (2012), Experimental Observation of Chimeras in Coupled-Map Lattices, Nature Physics 8, 658. (Cited on pages 27 and 215.)

Haim, D., Lev, O., Pismen, L. M., And Sheintuch, M. (1992), Modeling periodic and chaotic dynamics in anodic nickel dissolution, J. Phys. Chem. 96, 2676. (Cited on page 121.)

Hata, S., NakaO, H., AND Mikhailov, A. S. (2012), Global feedback control of Turing patterns in network-organized activator-inhibitor systems, Europhys. Lett. 98, 64004. (Cited on page 215.)

Hata, S., NakaO, H., And Mikhailov, A. S. (2014), Dispersal-induced destabilization of metapopulations and oscillatory Turing patterns in ecological networks, Scientific Reports 4, 3585. (Cited on page 215.)

Heвв, D. (1949), The Organization of Behavior: A Neuropsychological Theory, Wiley, New York, new edition edition, ISBN 0805843000. (Cited on page 185.) 
Heiligenthal, S., Dahms, T., Yanchuk, S., Jüngling, T., Flunkert, V., Kanter, I., SCHÖLl, E., AND KINZEL, W. (2011), Strong and Weak Chaos in Nonlinear Networks with Time-Delayed Couplings, Phys. Rev. Lett. 107, 234102. (Cited on pages 17 and 40.)

Heinrich, M., Dahms, T., Flunkert, V., Teitsworth, S. W., And Schöll, E. (2010), Symmetry breaking transitions in networks of nonlinear circuit elements, New J. Phys. 12, 113030. (Cited on page 210.)

Henderson, J. A. And Robinson, P. A. (2011), Geometric Effects on Complex Network Structure in the Cortex, Phys. Rev. Lett. 107, 018102. (Cited on page 15.)

Herrmann, G. (2001), A robust delay adaptation scheme for Pyragas' chaos control method, Phys. Lett. A 287, 245, ISSN 0375-9601. (Cited on page 159.)

Hethсоте, H. W. (2000), The mathematics of infectious diseases, SIAM review 42, 599. (Cited on page 32.)

Hilgetag, C. C., Burns, G. A., O’Neill, M. A., Scannell, J. W., And Young, M. P. (2000), Anatomical connectivity defines the organization of clusters of cortical areas in the macaque and the cat, Philos. trans. R. Soc. Lond., Ser. B 355, 91. (Cited on page 12.)

Hindmarsh, J. L. AND Rose, R. M. (1982), A model of the nerve impulse using two firstorder differential equations, Nature 296, 162. (Cited on page 49.)

Hindmarsh, J. L. AND Rose, R. M. (1984), A model of neuronal bursting using three coupled first order differential equations, Proc. Roy. Soc. B 221, 87. (Cited on page 49.)

Hinz, R., HÖVEL, P., AND SCHÖLL, E. (2011), Transient behavior in systems with timedelayed feedback, Chaos 21, 023114. (Cited on page 150.)

HizAnidis, J., Aust, R., AND SCHÖlL, E. (2008), Delay-induced multistability near a global bifurcation, Int. J. Bifur. Chaos 18, 1759. (Cited on pages 18, 47, 49 and 50.)

Hodgkin, A. L. (1948), The local electric changes associated with repetitive action in a medullated axon, J. Physiol. 107, 165. (Cited on pages 47 and 53.)

Hodgkin, A. L. AND HuXley, A. F. (1952), A Quantitative Description of Membrane Current and its Application to Conduction and Excitation in Nerve, J. Physiol. 117, 500. (Cited on page 53.)

Hopfield, J. J. (1982), Neural networks and physical systems with emergent collective computational abilities, Proc. Nat. Acad. Sci. U. S. A. 79, 2554. (Cited on page 184.) 
Hövel, P. (2009), Control of complex nonlinear systems with delay, Ph.D. thesis, Technische Universität Berlin. (Cited on pages 148 and 229.)

Hövel, P. (2010), Control of Complex Nonlinear Systems with Delay, Springer Theses, Springer, Heidelberg, ISBN 978-3-642-14109-6. (Cited on pages 32 and 105.)

HÖVEL, P. AND SCHÖLL, E. (2005), Control of unstable steady states by time-delayed feedback methods, Phys. Rev. E 72, 046203. (Cited on pages 17, 146, 147, 149, 150 and 151.)

Hu, G., Ditzinger, T., Ning, C. Z., AND HAKEn, H. (1993), Stochastic resonance without external periodic force, Phys. Rev. Lett. 71, 807. (Cited on pages 18, 47 and 49.)

Huepe, C., Zschaler, G., Do, A. L., AND Gross, T. (2011), Adaptive-network models of swarm dynamics, New J. Phys. 13, 073022. (Cited on page 184.)

Humphries, M. D., Gurney, K., And Prescott, T. J. (2006), The brainstem reticular formation is a small-world, not scale-free, network, Proceedings of the Royal Society B: Biological Sciences 273, 503. (Cited on page 12.)

Hunt, D., Korniss, G., AND Szymanski, B. K. (2010), Network Synchronization in a Noisy Environment with Time Delays: Fundamental Limits and Trade-Offs, Phys. Rev. Lett. 105, 068701. (Cited on page 32.)

IJSPEERT, A. (2008), Central pattern generators for locomotion control in animals and robots: A review, Neural Networks 21, 642, ISSN 0893-6080. (Cited on pages 14 and 67.)

IlLING, L., PANDA, C. D., AND SHARESHIAN, L. (2011), Isochronal chaos synchronization of delay-coupled optoelectronic oscillators, Phys. Rev. E 84, 016213. (Cited on pages 14, 67 and 70.$)$

INDIVERI, G., CHICCA, E., AND Douglas, R. (2006), A VLSI array of low-power spiking neurons and bistable synapses with spike-timing dependent plasticity, IEEE trans. neural netw. 17, 211. (Cited on page 90.)

ISELE, T. M. (2014), Excitation waves on complex networks, Ph.D. thesis, Technische Universität Berlin. (Cited on page 215.)

Isele, T. M., Hartung, B., Hövel, P., AND Schöll, E. (2015), Excitation Waves on a Minimal Small-World Model, Eur. Phys. J. B 88, 104, ISSN 1434-6028. (Cited on page 215.)

ISELE, T. M. AND SCHÖLL, E. (2015), Effect of small-world topology on wave propagation on networks of excitable elements, New J. Phys. 17, 023058. (Cited on page 215.) 
IZHIKEVICH, E. M. (2000a), Neural excitability, spiking and bursting, Int. J. Bifurcation Chaos 10, 1171. (Cited on page 47.)

IZHIKeVICH, E. M. (2000b), Neural Excitability, Spiking, and Bursting, Int. J. Bifurcation Chaos 10, 1171. (Cited on page 48.)

IZHIKEVICH, E. M. (2003), Simple model of spiking neurons, IEEE Trans. on Neur. Netw. 14, 1569. (Cited on page 92.)

IZHIKEVICH, E. M. (2004), Which model to use for cortical spiking neurons?, Neural Networks, IEEE Transactions on 15, 1063, ISSN 1045-9227. (Cited on page 92.)

IzHikevich, E. M. (2007), Dynamical Systems in Neuroscience, MIT Press, Cambridge, MA. (Cited on page 89.)

IZHIKEVICH, E. M. AND EDELMAN, G. M. (2008), Large-scale model of mammalian thalamocortical systems, Proc. Natl. Acad. Sci. U. S. A. 105, 3593. (Cited on page 90.)

Jahnke, S., Memmesheimer, R. M., And Timme, M. (2008), Stable Irregular Dynamics in Complex Neural Networks, Phys. Rev. Lett. 100, 048102. (Cited on page 32.)

Jeong, H., Mason, S. P., Barabasi, A. L., And Oltvai, Z. N. (2001), Lethality and centrality in protein networks, Nature 411, 41. (Cited on pages 12 and 31.)

Jeong, H., Tombor, B., Albert, R., Oltvai, Z. N., And Barabasi, A. L. (2000), The large-scale organization of metabolic networks, Nature 407, 651. (Cited on pages 12 and 21.)

JIRSA, V. K. (2008), Dispersion and time delay effects in synchronized spike-burst networks, Cogn. Neurodyn. 2, 29. (Cited on page 81.)

Jo, S. H., Chang, T., Ebong, I., Bhadviya, B. B., Mazumder, P., And Lu, W. (2010), Nanoscale memristor device as synapse in neuromorphic systems, Nano lett. 10, 1297. (Cited on page 90.)

Jolivet, F., R.and SchÜrmann, Berger, T. K., Naud, R., Gerstner, W., And Roth, A. (2008), The quantitative single-neuron modeling competition, Biol. cybern. 99, 417, ISSN 0340-1200. (Cited on page 94.)

JONAS, P. AND BuZSÁKI, G. (2007), Neural inhibition, Scholarpedia 2, 3286. (Cited on page 15.) 
Just, W., Bernard, T., Ostheimer, M., Reibold, E., And Benner, H. (1997), Mechanism of time-delayed feedback control, Phys. Rev. Lett. 78, 203. (Cited on pages 17 and 156.)

Just, W., Pelster, A., Schanz, M., And Schöll, E. (2010), Delayed Complex Systems, Theme Issue of Phil. Trans. R. Soc. A 368, ISSN 1364-503X. Pp.301-513. (Cited on page 17.)

Kaluza, P. And Mikhailov, A. S. (2014), Autonomous Learning by Simple Dynamical Systems with Delayed Feedbacks, Phys. Rev. E 90, 030901. 1406.7534. (Cited on page 214.)

Kandel, E., Schwartz, J. H., AND Jessell, T. M. (1996), Neurowissenschaften, Spektrum Akademischer Verlag, Heidelberg. (Cited on page 15.)

Kanter, I., Kopelowitz, E., Vardi, R., Zigzag, M., Kinzel, W., Abeles, M., And CoHEN, D. (2011a), Nonlocal mechanism for cluster synchronization in neural circuits, Europhys. Lett. 93, 66001. (Cited on pages 14, 67, 68, 69 and 183.)

Kanter, I., Zigzag, M., Englert, A., Geissler, F., And Kinzel, W. (2011b), Synchronization of unidirectional time delay chaotic networks and the greatest common divisor, Europhys. Lett. 93, 60003. (Cited on pages 14, 17, 67, 68, 69 and 183.)

KANTNER, M. AND YANCHUK, S. (2013), Bifurcation analysis of delay-induced patterns in a ring of Hodgkin-Huxley neurons, Phil. Trans. R. Soc. A 371, 20120470. (Cited on page 17.)

Keane, A., Dahms, T., Lehnert, J., Suryanarayana, S. A., Hövel, P., and Schöll, E. (2012), Synchronisation in networks of delay-coupled type-I excitable systems, Eur. Phys. J. B 85, 407, ISSN 1434-6028. (Cited on pages 13, 14, 15, 44, 50, 51, 52, 60, 61, 62, 63, 65, 184, 193, 223, 224 and 225.)

Kestler, J., Kinzel, W., AND Kanter, I. (2007), Sublattice synchronization of chaotic networks with delayed couplings, Phys. Rev. E 76, 035202. (Cited on pages 14 and 67.)

Kestler, J., Kopelowitz, E., Kanter, I., And Kinzel, W. (2008), Patterns of chaos synchronization, Phys. Rev. E 77, 046209. (Cited on pages 14, 17 and 67.)

KilPATRICK, Z. P. AND ERMENTROUT, B. (2011), Sparse gamma rhythms arising through clustering in adapting neuronal networks, PLOS comput. biol. 7, e1002281. (Cited on page 97.) 
Kinzel, W. (2013), Chaos in networks with time-delayed couplings, Phil. Trans. R. Soc. A 371, 20120461. (Cited on pages 17 and 40.)

Kinzel, W., Englert, A., Reents, G., Zigzag, M., And Kanter, I. (2009), Synchronization of networks of chaotic units with time-delayed couplings, Phys. Rev. E 79, 056207. (Cited on pages 13, 17, 40 and 184.)

KinZel, W. AND Kanter, I. (2008), Secure Communication with Chaos Synchronization, in E. Schöll and H. G. Schuster (Editors), Handbook of Chaos Control, Wiley-VCH, Weinheim. Second completely revised and enlarged edition. (Cited on page 17.)

Kiss, I. Z., Zhai, Y., AND Hudson, J. L. (2005), Predicting Mutual Entrainment of Oscillators with Experiment-Based Phase Models, Phys. Rev. Lett. 94, 248301. (Cited on page 121.)

Kittel, A., Parisi, J., And Pyragas, K. (1995), Delayed feedback control of chaos by selfadapted delay time, Phys. Lett. A 198, 433. (Cited on page 159.)

Kivelä, M., Arenas, A., Barthélemy, M., Gleeson, J. P., Moreno, Y., And Porter, M. A. (2014), Multilayer networks, Journal of Complex Networks 2, 203. (Cited on pages 12, 13 and 69.)

KocH, C. (1999), Biophysics of Computation: Information Processing in Single Neurons, Oxford University Press, New York. (Cited on pages 17 and 104.)

Kori, H. AND KuRAmoto, Y. (2001), Slow switching in globally coupled oscillators: robustness and occurrence through delayed coupling, Phys. Rev. E 63, 046214. (Cited on page 67.)

Koseska, A., Volkov, E., And KurThs, J. (2009), Detuning-dependent dominance of oscillation death in globally coupled synthetic genetic oscillators, Europhys. Lett. 85, 28002. (Cited on page 210.)

Koseska, A., Volkov, E., AND Kurths, J. (2010), Parameter mismatches and oscillation death in coupled oscillators, Chaos 20, 023132. (Cited on page 210.)

Koseska, A., Volkov, E., AND KurThs, J. (2013a), Oscillation quenching mechanisms: Amplitude vs. oscillation death, Phys. Rep. 531, 173. (Cited on page 210.)

Koseska, A., Volkov, E., AND Kurths, J. (2013b), Transition from Amplitude to Oscillation Death via Turing Bifurcation, Phys. Rev. Lett. 111, 024103. (Cited on page 210.) 
Kouvaris, N. E., Isele, T. M., Mikhailov, A. S., AND Schöll, E. (2014), Propagation failure of excitation waves on trees and random networks, EPL 106, 68001. (Cited on page 215.)

Krstic, M., Kanellakopoulos, I., and Kokotovic, P. (1995), Nonlinear and Adaptive Control Design, Wiley, New York. (Cited on page 142.)

Kuramoto, Y. (1984), Chemical Oscillations, Waves and Turbulence, Springer-Verlag, Berlin. (Cited on pages 17, 44 and 162.)

Kuramoto, Y. And Battogtokn, D. (2002), Coexistence of Coherence and Incoherence in Nonlocally Coupled Phase Oscillators., Nonlin. Phen. in Complex Sys. 5, 380. (Cited on pages 27 and 215.)

KyRYChKo, Y. N., Blyuss, K. B., AND SChÖLL, E. (2011), Amplitude death in systems of coupled oscillators with distributed-delay coupling, Eur. Phys. J. B 84, 307. (Cited on page 138.)

KyrychKo, Y. N., Blyuss, K. B., AND SChÖLL, E. (2013), Amplitude and phase dynamics in oscillators with distributed-delay coupling, Phil. Trans. R. Soc. A 371, 20120466. (Cited on page 138.)

KyRYchKo, Y. N., Blyuss, K. B., AND SCHÖLL, E. (2014), Synchronization of networks of oscillators with distributed-delay coupling, Chaos 24, 043117. (Cited on page 15.)

Ladenbauer, J., Augustin, M., Shiau, L., And Obermayer, K. (2012), Impact of Adaptation Currents on Synchronization of Coupled Exponential Integrate-and-Fire Neurons, PLoS Comput. Biol. 8, e1002478. ArXiv:1310.2430. (Cited on page 95.)

Ladenbauer, J., Lehnert, J., Rankoohi, H., Dahms, T., Schöll, E., and Obermayer, K. (2013), Adaptation controls synchrony and cluster states of coupled threshold-model neurons, Phys. Rev. E 88, 042713. (Cited on pages 14, 15, 89, 90, 91, 94, 96, 98 and 226.)

LANG, R. AND KOBAYASHI, K. (1980), External optical feedback effects on semiconductor injection laser properties, IEEE J. Quantum Electron. 16, 347. (Cited on page 70.)

LEHNERT, J. (2010), Dynamics of Neural Networks with Delay, Master's thesis, Technische Universität Berlin. (Cited on pages 14, 23, 28, 53, 55, 59, 68, 75 and 223.)

LEHNERT, J., DAHMS, T., HÖVEL, P., AND SCHÖLL, E. (2011a), Loss of synchronization in complex neural networks with delay, Europhys. Lett. 96, 60013. (Cited on pages 13, 14, 15, 55, 57, 58, 59, 63, 81, 84, 184, 193 and 224.) 
Lehnert, J., Hövel, P., Flunkert, V., Guzenko, P. Y., Fradkov, A. L., ANd Schöll, E. (2011b), Adaptive tuning of feedback gain in time-delayed feedback control, Chaos 21, 043111. (Cited on pages 16, 17, 145, 149, 151, 152, 153, 157, 158, 229 and 230.)

Lehnert, J., Hövel, P., Selivanov, A. A., Fradkov, A. L., ANd SchÖll, E. (2014), Controlling cluster synchronization by adapting the topology, Phys. Rev. E 90, 042914. (Cited on pages 16, 17, 185, 189, 193, 202, 203, 205, 206, 231, 232 and 233.)

Lentz, H. H. K., Selhorst, T., AND Sokolov, I. M. (2012), Spread of infectious diseases in directed and modular metapopulation networks, Phys. Rev. E 85, 066111. (Cited on page 32.)

Lewis, F. L., Vrabie, D., AND Syrmos, V. L. (2012), Optimal Control, EngineeringPro collection, Wiley, ISBN 9781118122723 . (Cited on page 214.)

Lichtner, M., Wolfrum, M., AND YANCHUK, S. (2011), The spectrum of delay differential equations with large delay, SIAM J. Math. Anal. 43, 788. (Cited on page 218.)

Lin, W., MA, H., Feng, J., AND Chen, G. (2010), Locating unstable periodic orbits: When adaptation integrates into delayed feedback control, Phys. Rev. E 82, 046214. (Cited on page 159.)

Lindner, B., García-Ojalvo, J., Neiman, A. B., and Schimansky-Geier, L. (2004), Effects of noise in excitable systems, Phys. Rep. 392, 321. (Cited on pages 47 and 104.)

Litwin-Kumar, A. And Doiron, B. (2012), Slow dynamics and high variability in balanced cortical networks with clustered connections, Nat. Neurosci. 15, 1498, ISSN 10976256. 10.1038/nn.3220. (Cited on page 90.)

LU, X. AND QIN, B. (2009), Adaptive cluster synchronization in complex dynamical networks, Phys. Lett. A 373, 3650, ISSN 0375-9601. (Cited on page 185.)

LÜcken, L., Pade, J. P., Knauer, K., And Yanchuk, S. (2013), Reduction of interaction delays in networks, EPL 103, 10006. (Cited on pages 17 and 103.)

LÜCKEN, L. AND YANCHUK, S. (2012), Two-cluster bifurcations in systems of globally pulse-coupled oscillators, Physica D 241, 350, ISSN 0167-2789. (Cited on pages 14 and 67.)

LÜDGE, K. (2012), Nonlinear Laser Dynamics - From Quantum Dots to Cryptography, Wiley-VCH, Weinheim, ISBN 978-3-527-41100-9. (Cited on page 17.)

Mantegna, R. N. (1999), Hierarchical structure in financial markets, The European physical journal. B 11, 193, ISSN 1434-6028. (Cited on page 13.) 
MASOller, C. AND ATAY, F. M. (2011), Complex transitions to synchronization in delaycoupled networks of logistic maps, Eur. Phys. J. D 62, 119. (Cited on page 137.)

Mikhailov, A. S., Davydov, V. A., And ZyKov, V. S. (1994), Complex dynamics of spiral waves and motion of curves, Phys. D 70, 1, ISSN 0167-2789. (Cited on page 47.)

Miyano, T. And Tsutsui, T. (2007), Data Synchronization in a Network of Coupled Phase Oscillators, Phys. Rev. Lett. 98, 024102. (Cited on page 13.)

Monasson, R. (1999), Diffusion, localization and dispersion relations on"small-world" lattices, Eur. Phys. J. B 12, 555. (Cited on pages 12, 21 and 106.)

Montoya, J. M. And SolÉ, R. V. (2002), Small world patterns in food webs, J. Theor. Biol. 214(3), 405. (Cited on pages 12 and 21.)

MORRIS, C. AND LECAR, H. (1981), Voltage oscillations in the barnacle giant muscle fiber., Biophys. J. 35, 193. (Cited on pages 49 and 53.)

Mosekilde, E., Maistrenko, Y., And Postnov, D. (2002), Chaotic Synchronization: Applications to Living Systems, World Scientific, Singapore. (Cited on pages 13, 14 and 67.)

Murthy, V. N. AND FetZ, E. E. (1996), Oscillatory activity in sensorimotor cortex of awake monkeys: synchronization of local field potentials and relation to behavior, J. neurophysiol. 76, 3949, ISSN 0022-3077. (Cited on page 13.)

Nagumo, J., Arimoto, S., And Yoshizawa., S. (1962), An active pulse transmission line simulating nerve axon., Proc. IRE 50, 2061. (Cited on pages 18, 47, 53 and 104.)

NaKajima, H., Ito, H., AND UedA, Y. (1997), Automatic Adjustment of Delay Time and Feedback Gain in Delayed Feedback Control of Chaos, Trans. Fundam. Electron. Commun. Comput. Sci. 80, 1554, ISSN 09168508. (Cited on page 159.)

NAKAO, H. AND MiKHAILOV, A. S. (2010), Turing patterns in network-organized activatorinhibitor systems, Nature Physics 6, 544. (Cited on page 215.)

Naud, R., Marcille, N., Clopath, C., and Gerstner, W. (2008), Firing patterns in the adaptive exponential integrate-and-fire model, Biol. cybern. 99, 335, ISSN 0340-1200. (Cited on pages 94, 97 and 100.)

NeWman, M. E. J. (2003), The Structure and Function of Complex Networks, SIAM Review 45, 167. (Cited on pages 11, 23, 30 and 184.) 
Newman, M. E. J. (2010), Networks: an introduction, Oxford University Press, Inc., New York. (Cited on pages 11 and 23.)

Newman, M. E. J., Barabasi, A. L., And Watts, D. J. (2006), The Structure and Dynamics of Networks, Princeton University Press, Princeton, USA, ISBN 0-691-11357-2. (Cited on pages 11 and 23.)

NeWman, M. E. J. AND WATTS, D. J. (1999), Renormalization group analysis of the smallworld network model, Phys. Lett. A 263, 341, ISSN 0375-9601. (Cited on pages 12 and 106.)

OKun, M. AND LAMPL, I. (2009), Balance of excitation and inhibition, Scholarpedia 4, 7467. (Cited on page 15.)

Olfati-SABer, R. AND MurRay, R. M. (2004), Consensus problems in networks of agents with switching topology and time-delays, IEEE Trans. Autom. Control 49, 1520, ISSN 0018-9286. (Cited on page 13.)

Omelchenko, I., Maistrenko, Y., Hövel, P., And SchölL, E. (2011), Loss of coherence in dynamical networks: spatial chaos and chimera states, Phys. Rev. Lett. 106, 234102. (Cited on pages 27 and 215.)

Omelchenko, I., Omel'chenko, O. E., Hövel, P., AND Schöll, E. (2013), When nonlocal coupling between oscillators becomes stronger: patched synchrony or multichimera states, Phys. Rev. Lett. 110, 224101. (Cited on pages 27 and 215.)

Omelchenko, I., Provata, A., Hizanidis, J., Schöll, E., And Hövel, P. (2015), Robustness of chimera states for coupled FitzHugh-Nagumo oscillators, Phys. Rev. E 91, 022917. (Cited on pages 27 and 215.)

Omelchenko, I., Riemenschneider, B., Hövel, P., Maistrenko, Y., And Schöll, E. (2012), Transition from spatial coherence to incoherence in coupled chaotic systems, Phys. Rev. E 85, 026212. (Cited on pages 27 and 215.)

Omelchenko, I., Rosenblum, M., And Pikovsky, A. (2010), Synchronization of slowfast systems, Eur. Phys. J. ST 191, 3. (Cited on page 13.)

Ott, E., Grebogi, C., And Yorke, J. A. (1990), Controlling Chaos, Phys. Rev. Lett. 64, 1196. (Cited on page 145.)

Oтто, C. (2013), Dynamics of Quantum Dot Lasers: Effects of Optical Feedback and External Optical Injection, Ph.D. thesis, TU Berlin. (Cited on page 17.) 
Panchuk, A., Rosin, D. P., Hövel, P., AND Schöll, E. (2013), Synchronization of coupled neural oscillators with heterogeneous delays, Int. J. Bifurcation Chaos 23, 1330039. (Cited on pages 83, 101, 115, 116 and 137.)

Parshani, R., Buldyrev, S. V., AND HAVlin, S. (2010), Interdependent Networks: Reducing the Coupling Strength Leads to a Change from a First to Second Order Percolation Transition, Phys. Rev. Lett. 105, 048701. (Cited on page 12.)

Pecora, L. M. (1998), Synchronization conditions and desynchronizing patterns in coupled limit-cycle and chaotic systems, Phys. Rev. E 58, 347. (Cited on page 37.)

Pecora, L. M. And Carroll, T. L. (1998), Master Stability Functions for Synchronized Coupled Systems, Phys. Rev. Lett. 80, 2109. (Cited on pages 3, 4, 13, 14, 32, 37, 39, 40, 42, 172, 193 and 211.)

Pecora, L. M., Sorrentino, F., Hagerstrom, A. M., Murphy, T. E., and Roy, R. (2014), Symmetries, Cluster Synchronization, and Isolated Desynchronization in Complex Networks, Nat. Commun. 5, 4079. (Cited on pages 14, 15, 67, 68 and 183.)

Pikovsky, A., Rosenblum, M. G., And Kurths, J. (2001), Synchronization, A Universal Concept in Nonlinear Sciences, Cambridge University Press, Cambridge. (Cited on page 13.)

Plotnikov, S. A., Lehnert, J., Fradkov, A. L., And Schöll, E. (2015), Control of synchronization in delay-coupled neural networks of heterogeneous nodes, arXiv 1503.07657v1. (Cited on pages 159 and 214.)

Pluchino, A., Latora, V., AND RapisARDA, A. (2005), Changing opinions in a changing world: A new perspective in sociophysics, International Journal of Modern Physics C 16, 515. Cited By 29. (Cited on page 13.)

Poeck, K. And Hacke, W. (2001), Neurologie, Springer, Heidelberg, 11th edition. (Cited on pages 14 and 15.)

Poel, W., Zakharova, A., ANd Schöll, E. (2015), Partial synchronization and partial amplitude death in mesoscale network motifs, Phys. Rev. E 91, 022915. (Cited on pages 14 and 67.)

Polyak, B. T. (2005), Stabilizing Chaos with Predictive Control, Automat. Rem. Contr. 66, 1791, ISSN 0005-1179. (Cited on page 159.)

Popovych, O. V., Yanchuk, S., And Tass, P. A. (2011), Delay- and Coupling-Induced Firing Patterns in Oscillatory Neural Loops, Phys. Rev. Lett. 107, 228102. (Cited on pages 17,32 and 215.) 
Popovych, O. V., YANCHUK, S., AND TASS, P. A. (2013), Self-organized noise resistance of oscillatory neural networks with spike timing-dependent plasticity, Sci. Rep. 3. (Cited on page 32.)

Pyragas, K. (1992), Continuous control of chaos by self-controlling feedback, Phys. Lett. A 170, 421. (Cited on pages 17, 145 and 155.)

Pyragas, K. and Pyragiene, T. (2008), Coupling design for a long-term anticipating synchronization of chaos, Phys. Rev. E 78, 046217. (Cited on page 161.)

Pyragas, V. And Pyragas, K. (2011), Adaptive modification of the delayed feedback control algorithm with a continuously varying time delay, Phys. Lett. A 375, 3866. (Cited on page 159.)

Pyragas, V. AND Pyragas, K. A. (2013), Adaptive search for the optimal feedback gain of time-delayed feedback controlled systems in the presence of noise, Eur. J. Phys. B 86, 1, ISSN 1434-6028. (Cited on page 159.)

Ramirez-Pastor, A. J., Riccardo, J. L., and Pereyra, V. D. (1998), Monte Carlo study of dimer adsorption at monolayer at square lattices, Surf. Sci. 411, 294. (Cited on page 137.)

RAPOPORT, A. (1957), Contribution to the theory of random and biased nets, Bull. Math. Biol. 19, 257. (Cited on pages 11,21, 29, 106 and 184.)

RAVASZ, E. AND BARABASI, A. L. (2003), Hierarchical organization in complex networks, Phys. Rev. E 67, 026112. (Cited on page 12.)

Rheinwalt, A., Goswami, B., Boers, N., Heitzig, J., Marwan, N., and Kurths, J. (2014), A Network of Networks Approach to Investigate the Influence of Sea Surface Temperature Variability on Monsoon Systems, in EGU General Assembly Conference Abstracts, volume 16, page 8147. (Cited on page 12.)

Rinzel, J. AND ERmentrout, G. B. (1989), Analysis of neural excitability and oscillations, in C. Koch and I. Segev (Editors), Methods in neuronal modeling, pages 251-291, MIT Press, Cambridge, MA. (Cited on page 47.)

Roelfsema, P., Engel, A., KöNig, P., AND Singer, W. (1997), Visuomotor integration is associated with zero time-lag synchronization among cortical areas, Nature 385, 157. (Cited on page 14.)

Rohden, M., Sorge, A., Timme, M., And Witthaut, D. (2012), Self-Organized Synchronization in Decentralized Power Grids, Phys. Rev. Lett. 109, 064101. (Cited on page 13.) 
Rosato, V., Issacharoff, L., Tiriticco, F., Meloni, S., Porcellinis, S., and Setola, R. (2008), Modelling interdependent infrastructures using interacting dynamical models, Int. J. Crit. Infrastruct. 4, 63. (Cited on page 12.)

Rosenblum, M. G. AND Pikovsky, A. (2004), Controlling Synchronization in an Ensemble of Globally Coupled Oscillators, Phys. Rev. Lett. 92, 114102. (Cited on pages 17 and 146.)

Rosenblum, M. G., Pikovsky, A., And Kurths, J. (1996), Phase Synchronization of Chaotic Oscillators, Phys. Rev. Lett. 76, 1804. (Cited on page 13.)

Rosenblum, M. G., Pikovsky, A., Kurths, J., SchäFer, C., ANd Tass, P. A. (2001), Phase synchronization: from theory to data analysis, Handbook of Biological Physics, volume 4, chapter 9, pages 279-321, Elsevier Science, Amsterdam, 1st edition. (Cited on page 44.)

Rosin, D. P. (2015), Dynamics of Complex Autonomous Boolean Networks, Springer Theses, Springer, Heidelberg, ISBN 978-3-319-13577-9. (Cited on pages 14 and 67.)

Rosin, D. P., Callan, K. E., Gauthier, D. J., AND SchÖll, E. (2011), Pulse-train solutions and excitability in an optoelectronic oscillator, Europhys. Lett. 96, 34001. (Cited on page 47.)

Rosin, D. P., Hövel, P., DAhlem, M. A., Kaiser, C., Kinzel, W., And Schöll, E. (2010), Neural bursting in discrete and continuous models with delay, in preparation. (Cited on page 17.)

Rosin, D. P., Rontani, D., Gauthier, D. J., And Schöll, E. (2012), Excitability in autonomous Boolean networks, Europhys. Lett. 100, 30003. (Cited on page 47.)

Rosin, D. P., Rontani, D., Gauthier, D. J., AND Schöll, E. (2013), Control of synchronization patterns in neural-like Boolean networks, Phys. Rev. Lett. 110, 104102. (Cited on page 67.)

Rossoni, E., Chen, Y., Ding, M., AND Feng, J. (2005), Stability of synchronous oscillations in a system of Hodgkin-Huxley neurons with delayed diffusive and pulsed coupling, Phys. Rev. E 71, 061904. (Cited on page 14.)

Rusin, C. G., Kori, H., Kiss, I. Z., AND Hudson, J. L. (2010), Synchronization engineering: tuning the phase relationship between dissimilar oscillators using nonlinear feedback, Phil. Trans. R. Soc. A 368, 2189, ISSN 1364-503X. (Cited on page 124.) 
Schikora, S., Hövel, P., WÜnsche, H. J., Schöll, E., ANd Henneberger, F. (2006), All-optical noninvasive control of unstable steady states in a semiconductor laser, Phys. Rev. Lett. 97, 213902. (Cited on page 161.)

SChikora, S., WÜnsche, H. J., AND Henneberger, F. (2011), Odd-number theorem: Optical feedback control at a subcritical Hopf bifurcation in a semiconductor laser, Phys. Rev. E 83, 026203. (Cited on page 161.)

SCHÖLL, E. (2013), Synchronization in delay-coupled complex networks, in Advances in Analysis and Control of Time-Delayed Dynamical Systems, Ed. by J.-Q. Sun, Q. Ding, chapter 4, pages 57-83, World Scientific, Singapore. (Cited on pages 13 and 15.)

Schöll, E., Hiller, G., Hövel, P., And Dahlem, M. A. (2009), Time-delayed feedback in neurosystems, Phil. Trans. R. Soc. A 367, 1079. (Cited on pages 54, 55, 64, 83, 115, 137 and 224.)

Schöll, E., Lehnert, J., Dahms, T., Selivanov, A. A., And Fradkov, A. L. (2012a), Adaptive time-delayed feedback control, in Proc. 2012 Internat. Symposium on Nonlinear Theory and its Applications (NOLTA2012), Palma de Mallorca, pages 674-677, IEICE, Japan. (Cited on pages 16 and 161.)

Schöll, E., Lehnert, J., Keane, A., Dahms, T., And Hövel, P. (2014), Control of desynchronization transitions in delay-coupled networks of type-I and type-II excitable systems, Proc. of the International Symposium, Hanse Institute of Advanced Studies, Delmenhorst, 13-16 November, 2012, in A. Pelster and G. Wunner (Editors), Selforganization in Complex Systems: The Past, Present, and Future of Synergetics, Springer, Berlin. (Cited on page 15.)

SCHÖLL, E. AND Schuster, H. G. (Editors) (2008), Handbook of Chaos Control, Wiley$\mathrm{VCH}$, Weinheim. Second completely revised and enlarged edition. (Cited on pages 17 and 146.)

Schöll, E., Selivanov, A. A., Lehnert, J., Dahms, T., Hövel, P., and Fradkov, A. L. (2012b), Control of synchronization in delay-coupled networks, Int. J. Mod. Phys. B 26, 1246007. (Cited on pages 16, 161, 162 and 178.)

SCHWARze, A. C. U. (2014), Controlling Desynchronization in Complex Network Topologies by the Balance of Excitation and Inhibition, Master's thesis, Technische Universität Berlin. (Cited on pages 56, 63 and 65.)

Selivanov, A., Lehnert, J., Fradkov, A. L., And Schöll, E. (2015), Adaptive timedelayed stabilization of steady states and periodic orbits, Phys. Rev. E 91, 012906. (Cited on pages 142 and 159.) 
Selivanov, A. A., Lehnert, J., Dahms, T., Hövel, P., Fradkov, A. L., AND Schöll, E. (2012), Adaptive synchronization in delay-coupled networks of Stuart-Landau oscillators, Phys. Rev. E 85, 016201. (Cited on pages 16, 159, 161, 162, 163, 164, 165, 166, 167, 181, 230 and 231.)

Sethia, G. C., Sen, A., And Atay, F. M. (2008), Clustered Chimera States in DelayCoupled Oscillator Systems, Phys. Rev. Lett. 100, 144102. (Cited on pages 27 and 215.)

Shefi, O., Golding, I., Segev, R., Ben-Jacob, E., And Ayali, A. (2002), Morphological characterization of in vitro neuronal networks, Phys. Rev. E 66, 021905. (Cited on page 12.)

Shiriaev, A. S. AND Fradkov, A. L. (2000), Stabilization of invariant sets for nonlinear non-affine systems, Automatica 36, 1709, ISSN 0005-1098. (Cited on page 144.)

Singer, W. (1999), Neuronal Synchrony: A Versatile Code Review for the Definition of Relations?, Neuron 24, 49. (Cited on page 13.)

SINGER, W. (2007), Binding by synchrony, Scholarpedia 2, 1657. (Cited on page 13.)

Socolar, J. E. S., Sukow, D. W., AND Gauthier, D. J. (1994), Stabilizing unstable periodic orbits in fast dynamical systems, Phys. Rev. E 50, 3245. (Cited on pages 146 and 154.)

SOlOMONOFF, R. AND RAPOPORT, A. (1951), Connectivety of random nets, Bull. Math. Biol. 13, 107. (Cited on pages 11, 21, 29 and 106.)

SONNENSCHEIN, J. (2013), Synchrony in delay-coupled excitable networks of type I and II, Bachelor Thesis, TU Berlin. (Cited on page 65.)

Soriano, M. C., García-Ojalvo, J., Mirasso, C. R., And Fischer, I. (2013), Complex photonics: Dynamics and applications of delay-coupled semiconductors lasers, Rev. Mod. Phys. 85, 421. (Cited on page 17.)

SORRENTINO, F. (2014), Group synchrony, parameter mismatches, and intragroup connections, to be published. (Cited on pages 14, 32 and 67.)

Sorrentino, F. And OtT, E. (2007), Network synchronization of groups, Phys. Rev. E 76, 056114. (Cited on pages 13, 14, 67, 68, 72, 81, 93 and 184.)

Sporns, O., Tononi, G., And Edelman, G. M. (2000), Theoretical Neuroanatomy: Relating Anatomical and Functional Connectivity in Graphs and Cortical Connection Matrices, Cereb. Cortex 10, 127. (Cited on page 12.) 
SPORNS, O. AND ZWI, J. (2004), The small world of the cerebral cortex, Neuroinformatics 2, 145, ISSN 1539-2791. (Cited on page 12.)

Strogatz, S. H. (2000), From Kuramoto to Crawford: exploring the onset of synchronization in populations of coupled oscillators, Physica D 143, 1. (Cited on page 104.)

Strogatz, S. H. And Stewart, I. (1993), Coupled Oscillators and Biological Synchronization, Sci. Am. 269, 102. (Cited on page 13.)

Sun, J., Bollt, E. M., AND NishiKaWA, T. (2009), Master stability functions for coupled nearly identical dynamical systems, Europhys. Lett. 85, 60011. (Cited on page 104.)

Timme, M., Geisel, T., AND Wolf, F. (2006), Speed of synchronization in complex networks of neural oscillators: Analytic results based on Random Matrix Theory, Chaos 16, 015108. (Cited on page 32.)

Touboul, J. AND BRetTe, R. (2008), Dynamics and bifurcations of the adaptive exponential integrate-and-fire model, Biol. cybern. 99, 319, ISSN 0340-1200. (Cited on page 94.)

UhlhaAs, P., Pipa, G., Lima, B., Melloni, L., Neuenschwander, S., Nikolic, D., AND SINGER, W. (2009), Neural synchrony in cortical networks: history, concept and current status, Front. Integr. Neurosci. 3, 17, ISSN 1662-5145. (Cited on pages 13 and 14.)

Vogels, T. P. AND АввотT, L. F. (2009), Gating multiple signals through detailed balance of excitation and inhibition in spiking networks, Nature Neurosci. 12, 483. (Cited on pages 15, 32 and 90.)

Vogels, T. P., Sprekeler, H., Zenke, F., Clopath, C., And Gerstner, W. (2011), Inhibitory Plasticity Balances Excitation and Inhibition in Sensory Pathways and Memory Networks, Science 334, 1569. (Cited on page 32.)

Vüllings, A., Hizanidis, J., Omelchenko, I., AND Hövel, P. (2014), Clustered Chimera States in Systems of Type-I Excitability, New J. Phys. 16, 123039. (Cited on pages 27 and 215.)

WANG, X. F. AND Chen, G. (2002), Synchronization in scale-free dynamical networks: robustness and fragility, IEEE trans. circuits syst. I, Fundam. theory appl. 49, 54. (Cited on page 13.)

WANG, X.-J. AND BUZSÁKI, G. (1996), Gamma Oscillation by Synaptic Inhibition in a Hippocampal Interneuronal Network Model, J. neurosci. 16, 6402. (Cited on page 49.)

Watts, D. J. AND Strogatz, S. H. (1998), Collective dynamics of 'small-world' networks, Nature 393, 440. (Cited on pages 12, 21, 30, 31, 33, 106, 184 and 223.) 
Wiedermann, M., Donges, J. F., Donner, R. V., And Kurths, J. (2014), Oceanatmosphere coupling from a climate network perspective, in EGU General Assembly Conference Abstracts, volume 16, page 11900. (Cited on page 12.)

Wigner, E. P. (1958), On the distribution of the roots of certain symmetric matrices, Ann. Math. 67, 325. (Cited on pages 29 and 63.)

Wille, C., LehNeRT, J., AND SCHÖLL, E. (2014), Synchronization-desynchronization transitions in complex networks: An interplay of distributed time delay and inhibitory nodes, Phys. Rev. E 90, 032908. (Cited on pages 15, 65 and 66.)

Williams, C. R. S., Murphy, T. E., Roy, R., Sorrentino, F., Dahms, T., AND SchöLl, E. (2013a), Experimental Observations of Group Synchrony in a System of Chaotic Optoelectronic Oscillators, Phys. Rev. Lett. 110, 064104. (Cited on pages 14 and 67.)

Williams, C. R. S., Sorrentino, F., Murphy, T. E., And Roy, R. (2013b), Synchronization states and multistability in a ring of periodic oscillators: Experimentally variable coupling delays, Chaos 23, 043117. (Cited on page 67.)

Williams, C. R. S., Sorrentino, F., Murphy, T. E., Roy, R., Dahms, T., And Schöll, E. (2012), Group Synchrony in an Experimental System of Delay-coupled Optoelectronic Oscillators, in Proc. 2012 Internat. Symposium on Nonlinear Theory and its Applications (NOLTA2012), Palma de Mallorca, pages 70-73, IEICE, Japan. (Cited on pages 14 and 67.)

Witthaut, D. AND Timme, M. (2012), Braess's paradox in oscillator networks, desynchronization and power outage, New Journal of Physics 14, 083036. (Cited on page 13.)

Wolfrum, M. (2012), The Turing bifurcation in network systems: Collective patterns and single differentiated nodes, Physica D 241, 1351. (Cited on page 215.)

Wolfrum, M. And Omel'chenko, O. E. (2011), Chimera states are chaotic transients, Phys. Rev. E 84, 015201. (Cited on pages 27 and 215.)

Wolfrum, M., Omel'chenko, O. E., Yanchuk, S., And Maistrenko, Y. (2011), Spectral properties of chimera states, Chaos 21, 013112. (Cited on pages 27 and 215.)

WRIGHT, E. M. (1949), The linear difference-differential equation with constant coefficients, Proc. R. Soc. Edinburgh, Sect. A: Math. Phys. Sci. 62, 387. (Cited on page 178.)

Wright, E. M. (1955), A non-linear difference-differential equation, J. Reine Angew. Math. 194, 66. (Cited on page 178.) 
WÜnsche, H. J., Brox, O., Radziunas, M., And Henneberger, F. (2001), Excitability of a Semiconductor Laser by a Two-Mode Homoclinic Bifurcation, Phys. Rev. Lett. 88, 023901. (Cited on page 47.)

YAnchuk, S. And Perlikowski, P. (2009), Delay and periodicity, Phys. Rev. E 79, 04622. (Cited on page 200.)

Yanchuk, S., Perlikowski, P., Popovych, O. V., And Tass, P. A. (2011), Variability of spatio-temporal patterns in non-homogeneous rings of spiking neurons, Chaos 21, 047511. (Cited on page 215.)

YANG, Y. AND WANG, Z. (2008), Control of cardiac excitability by microRNAs, Cardiovas. res. 79, 571. (Cited on page 47.)

Ye, H., Michel, A. N., AND Hou, L. (1998), Stability theory for hybrid dynamical systems, IEEE trans. automat. contr. 43, 461, ISSN 0018-9286. (Cited on page 89.)

Zakharova, A., Kapeller, M., AND SCHÖll, E. (2014), Chimera Death: Symmetry Breaking in Dynamical Networks, Phys. Rev. Lett. 112, 154101. (Cited on pages 27 and 215.)

Zakharova, A., Schneider, I., Kyrychio, Y. N., Blyuss, K. B., Koseska, A., Fiedler, B., AND SCHÖLL, E. (2013), Time delay control of symmetry-breaking primary and secondary oscillation death, Europhys. Lett. 104, 50004. (Cited on page 210.)

ZigzaG, M., Butkovski, M., Englert, A., Kinzel, W., And KAnter, I. (2009), Zerolag synchronization of chaotic units with time-delayed couplings, Europhys. Lett. 85, 60005. (Cited on pages 13, 116 and 184.)

Zigzag, M., Butkovski, M., Englert, A., Kinzel, W., And Kanter, I. (2010), Zero-lag synchronization and multiple time delays in two coupled chaotic systems, Phys. Rev. E 81, 036215. (Cited on page 17.) 


\section{ACKNOWLEDGMENTS}

First of all, I would like to thank Prof. Eckehard Schöll for introducing me to the exciting field of network dynamics. I also thank him for his supervision, valuable help, and support by suggestions and questions regarding my research. I appreciate his encouragement to frequent visits of international conferences which enabled me to profit from the exchange of knowledge within the research community from a very early stage on.

I am very much in debt to Dr. Thomas Dahms and Dr. Philipp Hövel who provided guidance during the research and writing of this thesis.

I would like to thank Prof. Alexander Fradkov for introducing me to the speed gradient method on which the second part of this thesis is based. The collaboration with him, Dr. Peter Guzenko, Dr. Anton Selivanov and Sergei Plotnikov was very pleasant and stimulating. I am also grateful for their great hospitality during my research visits to St. Petersburg.

Furthermore, I would like to thank Prof. Klaus Obermayer and Josef Ladenbauer for the fruitful collaboration which resulted in some of the results presented in Chapter 5. Likewise, I am grateful for the collaboration with Prof. Jack Hudson and Karen Blaha, PhD. Their experimental findings stimulated new theoretical results which added to Chapter 6.

I owe thanks to all the current, former, and visiting members of the Schöll group for the wonderful working atmosphere. In particular, I thank Dr. Andreas Amann, Dr. Valentin Flunkert, Dr. Thomas Isele, Andrew Keane, Alice Schwarze, Johannes Pardowitz, Jonas Sonnenschein, Caglar Cakan, Carolin Wille, and Jakub Sawicki for exciting discussions and fruitful collaborations.

I would like to thank PD Dr. Serhiy Yanchuk for preparing the second assessment of this thesis and Prof. Stephan Reitzenstein for chairing the defense of this thesis. 
This work was supported in the framework of the Collaborative Research Center 910 Control of self-organizing nonlinear systems: Theoretical methods and concepts of application. Furthermore, I acknowledge the support by the German-Russian Interdisciplinary Science Center (G-RISC) funded by the German Federal Foreign Office via the German Academic Exchange Service (DAAD). This support enabled me to spend two research visits in the group of Prof. Alexander Fradkov in St. Petersburg which were crucial for this work. 


\section{INDEX}

adaptive control, 141

adaptive exponential integrate-and-fire, 94

adaptive networks, 184

adaptive time-delayed feedback control, 145

adaptive topologies, 183

all-to-all network, 28

approximate synchronization, 106

assortativity, 23

betweenness, 25

block diagonalization, 40

chaos, 156

characteristic equation, 217

chemical oscillators, 120

circulant matrices, 88

circulant matrix, 26

cluster synchronization, 67

clustering coefficient, 25

community structure, 24

commuting matrices, 79

complex coupling strength, 89

component, 24

conduction velocity, 104

control of several parameters, 178

control of a subset of links, 204

control of an $M$-cluster state, 164, 188 coupling phase, 89

degree distribution, 23

delay differential equation, 33

delay differential equations, 217

delay matrix, 105

delay modulated topology, 193

delayed coupling, 33

dihedral group, 76

drifting parameters, 141, 151

eigenspectra of small-world and random networks with inhibition, 59

electrochemical cell, 120

equal-row-sum condition, 39

Erdős-Rényi random networks, 29

excitability, 48

excitatory links, 188

extended time-delayed feedback control, 154

FitzHugh-Nagumo model, 104

FitzHugh-Nagumo model with heterogeneous delays, 104

FitzHugh-Nagumo system, 53

Floquet exponent, 88, 175, 178

gain matrix, 143

Gaussian white noise, 151 
generalized Kuramoto order parameter, 162

goal function for an M-cluster state, 162 goal function, 186

gradient methods, 143

group synchronization, 67

heterogeneities, 168

heterogenous delays, 104

highly synchronized spiking, 106

Hopf bifurcation, 86, 121

inhibitory links, 188

integrate-and-fire models, 89

Kronecker product, 219

Lambert function, 178

linear stability analysis, 168

linear stability analysis of the control of coupling phase, 168

longitudinal eigenvalue, 42

Lorenz system, 162

Lyapunov exponent, 41

Lyapunov-Krasovskii functional, 159

M-cluster state, 87

master stability function for non-smooths systems, 90

master stability equation, 42,172

master stability equation for non-smooth systems, 91

methods of steps, 33

model uncertainties, 141

multistability, 142, 175

Neimark-Sacker bifurcation, 156

node degree, 23

noise, 151

non-smooth systems, 89 nonidentical nodes, 168

nonlinear coupling, 127

optimal feedback gain, 158

oscillation death, 210

oscillation quenching, 200

partial amplitude death, 107

penalty terms, 162, 186

period-doubling bifurcation, 156

phase of the complex coupling strength, 161

primary states, 123

Pyragas control, 146

quantifying synchronization, 44

Rössler system, 156

random network, 204

random network with inhibition, 56

relaxation oscillation, 121

robustness of control, 191

row-wise discrete Fourier transform, 195

saddle-node bifurcation on invariant cycle, 48

saddle-node infinite period bifurcation, 48

scale-free networks, 31

secondary states, 127

shortest path length, 24

small-world network with inhibition, 56

small-world networks, 30

smooth oscillation, 121

SNIPER model, 49

speed gradient method, 142

speed of the goal function, 142

stabilization of an unstable fixed point, 146

Stuart-Landau oscillator, 85, 123, 164, 187 
supercritical Hopf, 104

supercritical Hopf bifurcation, 86

synchronization, 38

synchronization manifold, 38

synchronization-desynchronization tran-

sitions, 55

synchronized solution, 38

the Kuramoto order parameter, 44

time-delayed feedback control, 145

transcendental equation, 88

transpassive state, 121

transversal eigenvalue, 41

traveling disruptions, 106

type-I excitability, 48

type-II excitability, 51

unidirectional ring, 27

unity-row-sum condition, 39

unstable periodic orbits, 156

variational equation, 218

Watt-Strogatz model, 30 